Supporting Information

\title{
Direct Access to Mono-Protected Homoallylic 1,2-Diols via Dual Chromium/Photoredox Catalysis
}

Felix Schäfers, ${ }^{[a]}$ Linda Quach, ${ }^{[a]}$ J. Luca Schwarz, ${ }^{[a]}$ Mar Saladrigas, ${ }^{\text {[a] }}$ Constantin G. Daniliuc, ${ }^{\text {[a] }}$ and Frank Glorius ${ }^{[a] *}$

[a] Organisch-Chemisches Institut, Westfälische Wilhelms-Universität Münster, Corrensstrasse 40, 48149 Münster (Germany)

*E-Mail: glorius@uni-muenster.de 


\section{Table of Contents}

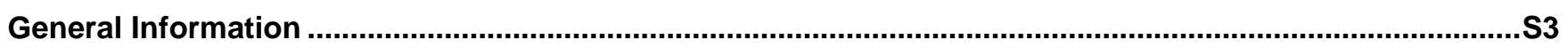

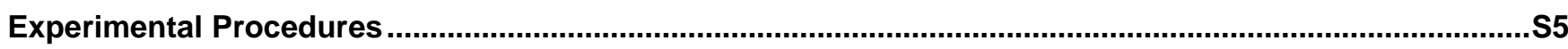

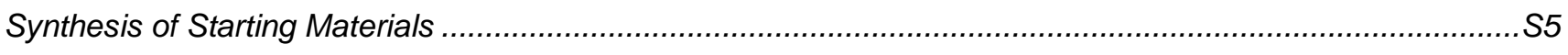

General Procedures for the Synthesis of Mono-Protected 1,2-Diols.......................................................

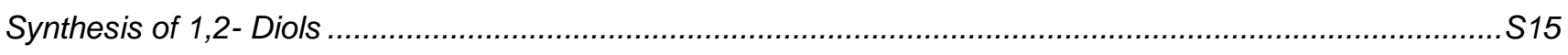

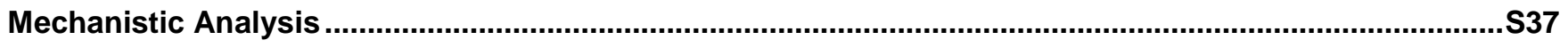

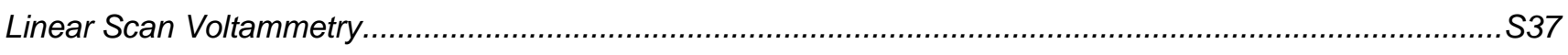

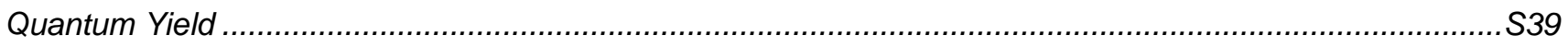

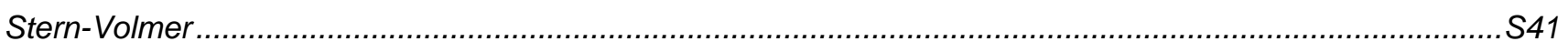

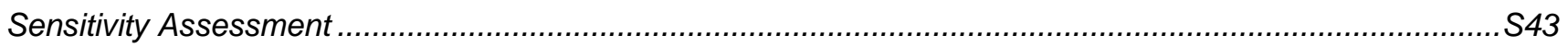

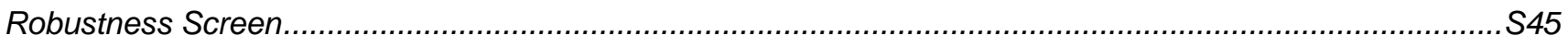

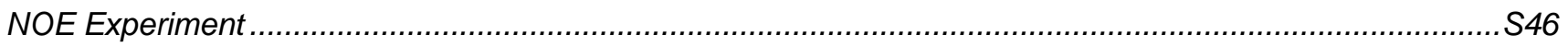

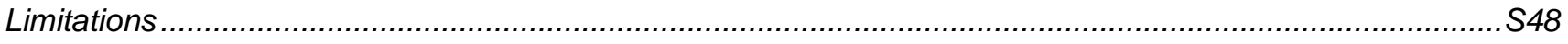

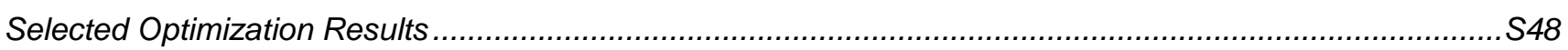

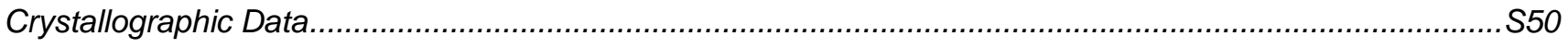

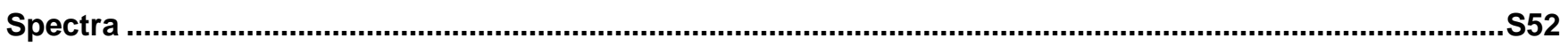

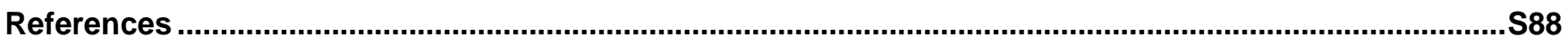

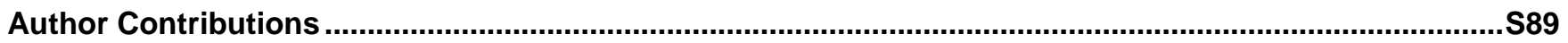




\section{General Information}

Unless otherwise noted, all reactions were carried out under an atmosphere of argon in ovendried glassware. The solvents used were either purified by distillation over standard drying agents and were stored over molecular sieves or directly used from a solvent purification system (HPLC grade, dried via an alumina column under a positive argon pressure). Reaction temperatures are reported as the temperature of the medium surrounding the vessel.

Photochemical reactions were performed in a Hepatochem EvoluChem ${ }^{\mathrm{TM}}$ PhotoRedOx Box Duo device (Figure S1) and irradiated with two EvoluChem ${ }^{\mathrm{TM}}$ P303-30-1 LEDs (30 W, $\lambda_{\max }=$ $450 \mathrm{~nm}$, Figure S2). To shield irradiation, the setup was covered with a small paper box. The reaction temperature was measured to be between $30^{\circ} \mathrm{C}$ and $35^{\circ} \mathrm{C}$ using this setup.
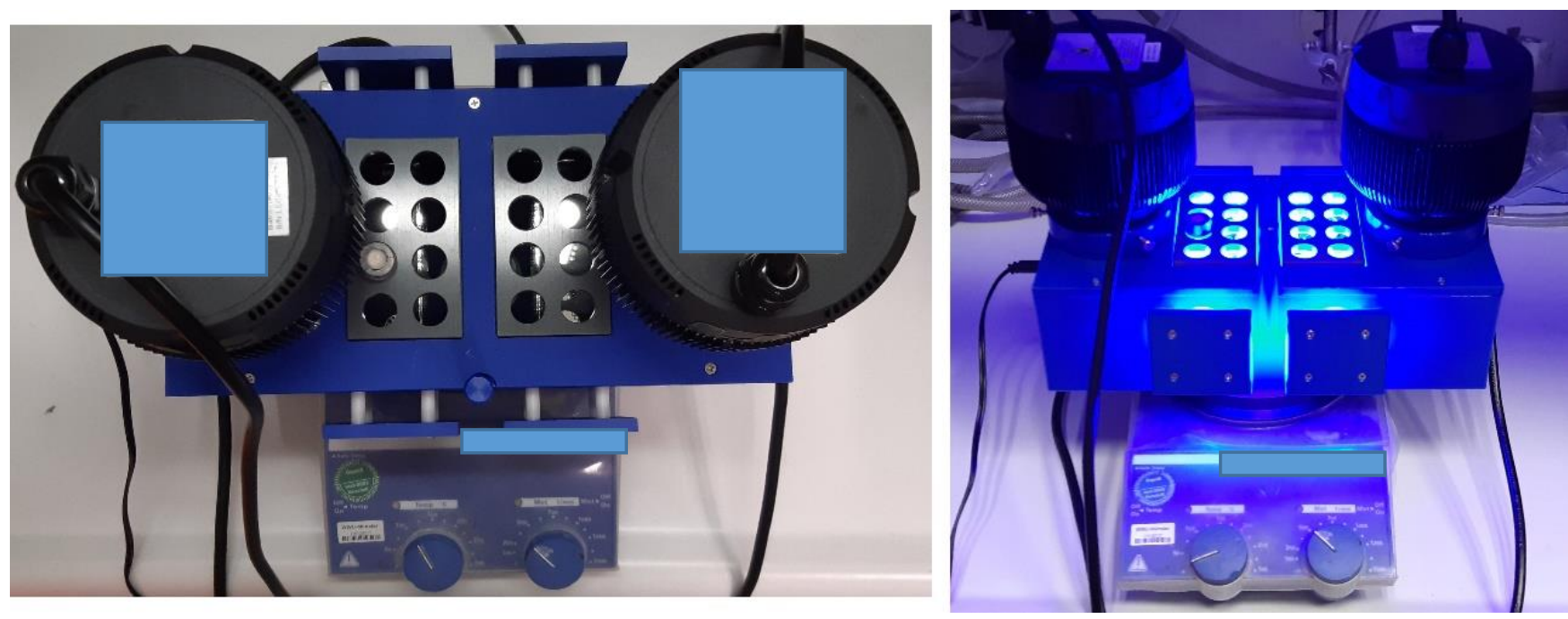

Figure S1. Hepatochem EvoluChem ${ }^{\mathrm{TM}}$ PhotoRedOx Box Duo device.

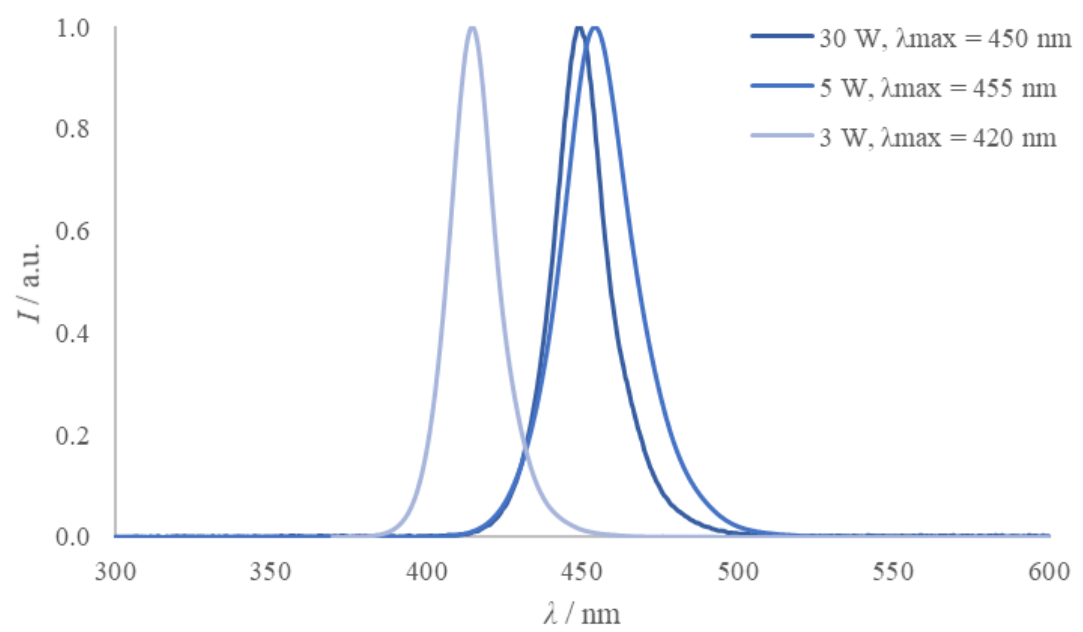

Figure S2. Emission spectrum of the EvoluChem ${ }^{\mathrm{TM}} \mathrm{P} 303-30-1$ LED $\left(30 \mathrm{~W}, \lambda_{\max }=450 \mathrm{~nm}\right)$ used for reaction irradiation, robustness screening and sensitivity assessment. 
Analytical thin layer chromatography (TLC) was performed on silica gel 60 F254 aluminum plates (Merck). TLC plates were visualized by exposure to short wave ultraviolet light (254 nm, $366 \mathrm{~nm})$ and/or were dipped into a solution of $\mathrm{KMnO}_{4}(1.00 \mathrm{~g}), \mathrm{K}_{2} \mathrm{CO}_{3}(6.00 \mathrm{~g})$ and $\mathrm{KOH}$ (100 mg) in $\mathrm{H}_{2} \mathrm{O}(100 \mathrm{~mL})$. Column chromatography was carried out using Acros Organics silica gel (35-70 mesh) eluting with the specified solvent system.

NMR-spectra were recorded on a Bruker Avance II 300, Bruker Avance II 400, Bruker Avance Neo 400, Agilent DD2 500 or on an Agilent DD2 600 spectrometer. Chemicals shifts ( $\delta$ ) are quoted in ppm downfield of tetramethylsilane. The residual solvent signals were used as references for ${ }^{1} \mathrm{H}$ and ${ }^{13} \mathrm{C}$ NMR spectra $\left(\mathrm{CDCl}_{3}: \delta_{\mathrm{H}}=7.26 \mathrm{ppm}, \delta_{\mathrm{C}}=77.16 \mathrm{ppm}, \mathrm{CD}_{2} \mathrm{Cl}_{2}: \delta_{\mathrm{H}}=\right.$ $\left.5.32 \mathrm{ppm}, \delta_{\mathrm{c}}=54.00 \mathrm{ppm}\right) .{ }^{19} \mathrm{~F} /{ }^{11} \mathrm{~B}$ NMR spectra are not calibrated by an internal reference. The multiplicity of all signals was described with standard abbreviations. Coupling constants $(\mathcal{J})$ are quoted in $\mathrm{Hz}$. Spectral data is typically reported for the major diastereomer. In case of a diastereomeric ratio $<60: 40$, the spectral data for both diasteromers is reported.

Samples for GC were filtered over a plug of silica with EtOAc before analysis if not stated otherwise. GC-MS spectra were recorded on an Agilent Technologies 7890A GC-system with an Agilent 5975C VL MSD or an Agilent 5975 inert Mass Selective Detector (EI) and a HP-5MS column $(0.25 \mathrm{~mm} \cdot 30 \mathrm{~m}$, film: $0.25 \mu \mathrm{m})$. GC-FID analysis was undertaken on an Agilent Technologies 6890A equipped with a HP-5 quartz column $(0.32 \mathrm{~mm} \cdot 30 \mathrm{~m}$, film: $0.25 \mu \mathrm{m})$ using flame ionization detection. High-resolution mass spectra (HRMS) were obtained by the MS service of the Organisch-Chemisches Institut, Westfälische Wilhelms-Universität Münster, using electrospray ionisation (ESI) on a Bruker Daltonics, MicroToF spectrometer.

Aldehyde starting materials were checked for purity by TLC analysis and distilled if needed. Commercial 2,6-lutidine was purified by distillation. All other commercially available chemicals were used as received if not stated otherwise.

The photocatalysts $\left[\operatorname{Ir}\left(\mathrm{dFCF}_{3} \mathrm{ppy}\right)_{2}-\left(5,5^{\prime}-\mathrm{dCF}_{3} \mathrm{bpy}\right)\right] \mathrm{PF} F_{6},\left[\operatorname{Ir}\left(\mathrm{dFCF}_{3} \text { ppy }\right)_{2}-\left(4,4^{\prime}-\mathrm{dCF}_{3} \mathrm{bpy}\right)\right] \mathrm{PF} \mathrm{F}_{6}$ and $\left[\mathrm{Ir}\left(\mathrm{dFCF}_{3} \mathrm{ppy}\right)_{2}-\left(4,4^{\prime}-\mathrm{dFbpy}\right)\right] \mathrm{PF}_{6}$ were prepared following literature procedures. ${ }^{1} a, b \quad \mathrm{CrCl}_{2}$ (99.9\% trace metal basis) was purchased from Alfa Aesar or Fluorochem and stored in an argon-filled glovebox at room temperature. $\mathrm{CrCl}_{3}$ (99\% trace metal basis) was purchased from Sigma-Aldrich and stored under air at room temperature. 


\section{Experimental Procedures}

\section{Synthesis of Starting Materials}

\section{Triisopropyl((2-methylprop-1-en-1-yl)oxy)silane (1b)}<smiles>CC(C)=CO[Si](C(C)C)(C(C)C)C(C)C</smiles>

Prepared following a modified literature procedure. ${ }^{2}$ Triethylamine $(1.57 \mathrm{~mL}$, $11.1 \mathrm{mmol}, 3.0$ equiv.) was added dropwise to a cooled $\left(0^{\circ} \mathrm{C}\right)$ solution of 2 methyl-propanal (340 $\mu \mathrm{L}, \quad 3.70 \mathrm{mmol}, 1.0$ equiv.) in $\mathrm{CH}_{2} \mathrm{Cl}_{2} \quad(7.5 \mathrm{~mL})$. Triisopropylsilyl trifluoromethanesulfonate $(1.00 \mathrm{~mL}, 3.70 \mathrm{mmol}, 1.0$ equiv.) was added dropwise at $0{ }^{\circ} \mathrm{C}$. The reaction mixture was allowed to warm up to room temperature, stirred

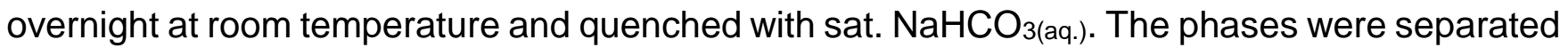
and the aqueous phase was extracted with $\mathrm{Et}_{2} \mathrm{O}$. The combined organic phases were dried over $\mathrm{MgSO}_{4}$, filtered over a short silica plug and concentrated under reduced pressure to give the product as a colorless oil (668 mg, $2.92 \mathrm{mmol}, 79 \%)$.

${ }^{1} \mathrm{H}$ NMR $\left(400 \mathrm{MHz}_{\mathrm{CDCl}}\right)$ : $\delta 6.23-6.03(\mathrm{~m}, 1 \mathrm{H}), 1.62(\mathrm{~m}, 3 \mathrm{H}), 1.53(\mathrm{~m}, 3 \mathrm{H}), 1.16-1.05(\mathrm{~m}$, $21 \mathrm{H})$;

${ }^{13} \mathrm{C}$ NMR (101 MHz, $\left.\mathrm{CDCl}_{3}\right): \delta 134.2,112.5,19.4,17.9,14.8,12.2$;

HRMS $\left(\mathrm{ESI}^{+}\right)$: [M+Na] $]^{+}$251.1807, found 251.1804;

$\mathbf{R}_{\mathbf{f}}($ pentane/Et $2 \mathrm{O}=98 / 2): 0.88$.

\section{tert-Butyldimethyl(prop-1-en-1-yloxy)silane}

following a modified literature procedure. ${ }^{3}$ tertstirred solution of 1,8-diazabicyclo[5.4.0]undec-7-en $(1.20 \mathrm{~mL}, 8.00 \mathrm{mmol}, 1.6$ equiv.) and propionaldehyde (360 $\mu \mathrm{L}, 5.00 \mathrm{mmol}, 1.0$ equiv.) in $\mathrm{CH}_{2} \mathrm{Cl}_{2}(7.5 \mathrm{~mL})$ and the reaction mixture was stirred overnight. The reaction mixture was quenched with dist. $\mathrm{H}_{2} \mathrm{O}$, the phases were separated and the aqueous phase was extracted with $\mathrm{CH}_{2} \mathrm{Cl}_{2}$. The combined organic phases 
were dried over $\mathrm{MgSO}_{4}$ and concentrated under reduced pressure. Purification by column chromatography on silica gel (pentane/ $\mathrm{Et}_{2} \mathrm{O}=98 / 2$ ) gave the product as a colorless oil ( $683 \mathrm{mg}$, $3.96 \mathrm{mmol}, 79 \%$ in a 50:50 E/Z-ratio.

${ }^{1} \mathrm{H}$ NMR $\left(400 \mathrm{MHz}, \mathrm{CDCl}_{3}\right): \delta 6.25-6.17(\mathrm{~m}, 1 \mathrm{H}), 4.98(\mathrm{~m}, 0.5 \mathrm{H}), 4.50(\mathrm{~m}, 0.5 \mathrm{H}), 1.57(\mathrm{dd}, J=$ 6.7, $1.7 \mathrm{~Hz}, 1.5 \mathrm{H}), 1.51(\mathrm{dd}, J=6.8,1.7 \mathrm{~Hz}, 1.5 \mathrm{H}), 0.93(\mathrm{~s}, 4.5 \mathrm{H}), 0.91(\mathrm{~s}, 4.5 \mathrm{H}), 0.13(\mathrm{~s}, 3 \mathrm{H})$, $0.12(\mathrm{~s}, 3 \mathrm{H})$;

${ }^{13} \mathrm{C}$ NMR $\left(101 \mathrm{MHz}, \mathrm{CDCl}_{3}\right): \delta$ 140.7, 139.4, 105.8, 105.0, 25.9, 25.8, 18.4, 15.4, 12.3, 9.1,-5.1, -5.2 ;

HRMS $\left(\mathrm{ESI}{ }^{+}\right):[2 \mathrm{M}+\mathrm{Na}]^{+} 367.2458$, found 367.2458 ;

$\mathbf{R}_{\mathbf{f}}\left(\right.$ pentane $\left./ \mathrm{Et}_{2} \mathrm{O}=98 / 2\right): 0.90$.

\section{Triisopropyl(prop-1-en-1-yloxy)silane}

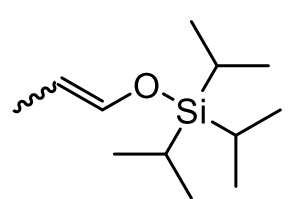

Prepared following a modified literature procedure. ${ }^{4}$ Triisopropylsilyl trifluoromethanesulfonate $(1.61 \mathrm{~mL}, 6.00 \mathrm{mmol}, 1.2$ equiv) was dissolved in $\mathrm{Et}_{2} \mathrm{O}(7.5 \mathrm{~mL})$ and cooled to $0^{\circ} \mathrm{C}$. Triethylamine $(836 \mu \mathrm{L}, 6.00 \mathrm{mmol}$, 1.2 equiv) was added and the mixture was stirred for $30 \mathrm{~min}$ at $0{ }^{\circ} \mathrm{C}$. Then, a solution of propionaldehyde ( $359 \mu \mathrm{L}, 5.00 \mathrm{mmol}, 1.0$ equiv.) in THF ( $2.5 \mathrm{~mL}$ ) was added dropwise to the reaction mixture. The reaction was allowed to warm to room temperature and stirred overnight. The reaction was quenched with dist. $\mathrm{H}_{2} \mathrm{O}$ and extracted with $\mathrm{Et}_{2} \mathrm{O}$. The combined organic phases were dried over $\mathrm{MgSO}_{4}$ and concentrated under reduced pressure. Purification by column chromatography on silica gel (pentane) gave the product as a colorless oil (990 mg, $4.62 \mathrm{mmol}, 77 \%$ ) in a $93: 7 \mathrm{E} / Z$-ratio.

${ }^{1} \mathrm{H}$ NMR $\left(400 \mathrm{MHz}, \mathrm{CDCl}_{3}\right) \delta 6.32(\mathrm{~m}, 0.07 \mathrm{H}), 6.29(\mathrm{~m}, 0.93 \mathrm{H}), 5.00(\mathrm{~m}, 0.07 \mathrm{H}), 4.45(\mathrm{~m}, 0.93 \mathrm{H})$, $1.59(\mathrm{dd}, J=6.7,1.8 \mathrm{~Hz}, 2.79 \mathrm{H}), 1.52(\mathrm{dd}, J=6.8,1.6 \mathrm{~Hz}, 0.21 \mathrm{H}), 1.21-1.11(\mathrm{~m}, 3 \mathrm{H}), 1.11-$ $1.04(\mathrm{~m}, 18 \mathrm{H})$; 
${ }^{13} \mathrm{C}$ NMR $\left(101 \mathrm{MHz}, \mathrm{CDCl}_{3}\right) \delta=141.1,139.9,105.5,104.3,17.9,12.1,9.0$;

HRMS $\left.(\mathrm{ESI})^{+}\right):[\mathrm{M}+\mathrm{Na}]^{+}$237.1645, found 237.1638;

$\mathbf{R}_{\mathbf{f}}($ pentane/Et $2 \mathrm{O}=97 / 3): 0.95$.

\section{1-((2-Methylprop-1-en-1-yl)oxy)butane}<smiles>CCCCOC=C(C)C</smiles>

Prepared following a modified literature procedure. ${ }^{5}$ 1-Butanol $(0.79 \mathrm{~mL}$, $8.63 \mathrm{mmol}, 1.1$ equiv.) was added dropwise to a suspension of $\mathrm{NaH}(374 \mathrm{mg}$, $60 \%$ in mineral oil, $9.35 \mathrm{mmol}$. 1.2 equiv. $)$ in THF $(8.0 \mathrm{~mL})$ at $0{ }^{\circ} \mathrm{C}$. The solution was allowed to warm to room temperature and 3-bromo-2-methylpropene $(0.79 \mathrm{~mL}, 7.84 \mathrm{mmol}, 1.0$ equiv.) was added before the reaction mixture was stirred overnight at $70{ }^{\circ} \mathrm{C}$. The reaction mixture was quenched with dist. $\mathrm{H}_{2} \mathrm{O}$, the phases were separated and the aqueous phase was extracted with $\mathrm{Et}_{2} \mathrm{O}$. The combined organic phases were filtered over a silica plug and concentrated under reduced pressure to give the crude allyl ether ( $660 \mathrm{mg}, 5.15 \mathrm{mmol}, 66 \%)$. A part of the crude allyl ether (300 mg, $2.34 \mathrm{mmol}, 1.0$ equiv.) was isomerized by dropwise addition to a freshly prepared solution of $\operatorname{LDA}\left(2.0\right.$ equiv.) in THF $(7 \mathrm{~mL})$ at $-78^{\circ} \mathrm{C}$. The reaction mixture was allowed to warm up to room temperature overnight and quenched with dist. $\mathrm{H}_{2} \mathrm{O}$. The phases were separated and the aqueous phase was extracted with $\mathrm{E}_{2} \mathrm{O}$. The combined organic phases were washed with brine, dried over $\mathrm{MgSO}_{4}$ and concentrated under reduced pressure. Purification by column chromatography on silica gel (pentane/Et2O $=95 / 5$ ) gave the product as a colorless oil (294 mg, $2.29 \mathrm{mmol}, 65 \%$ over two steps).

${ }^{1} \mathrm{H}$ NMR $\left(400 \mathrm{MHz}, \mathrm{CDCl}_{3}\right): \delta 5.78$ (hept, $\left.J=1.6 \mathrm{~Hz}, 1 \mathrm{H}\right), 3.65(\mathrm{~m}, 2 \mathrm{H}), 1.61(\mathrm{~s}, 3 \mathrm{H}), 1.60-1.55$ $(\mathrm{m}, 2 \mathrm{H}), 1.54(\mathrm{~s}, 3 \mathrm{H}), 1.43-1.34(\mathrm{~m}, 2 \mathrm{H}), 0.93(\mathrm{t}, J=7.6 \mathrm{~Hz}, 3 \mathrm{H})$;

${ }^{13} \mathrm{C}$ NMR (101 MHz, $\left.\mathrm{CDCl}_{3}\right): \delta$ 140.3, 110.3, 71.6, 32.0, 19.7, 19.3, 15.1, 14.0;

GC MS (EI): 128.1 (32), 72.0 (71), 57.1 (100), 43.1 (61), 41.1 (40);

$\mathbf{R}_{\mathbf{f}}($ pentane/EtOAc $=95 / 5): 0.85$. 


\section{(((2-Methylprop-1-en-1-yl)oxy)methyl)benzene}

Prepared following a literature procedure. ${ }^{6}$ To a suspension of Cul (95.0 mg, $500 \mu \mathrm{mol}, 10 \mathrm{~mol} \%$ ) and 3,4,7,8-tetramethyl-1,10-phenanthroline (236 mg, $1.00 \mathrm{mmol}, 20 \mathrm{~mol} \%$ ) in toluene $(5 \mathrm{~mL})$ were added $\mathrm{Cs}_{2} \mathrm{CO}_{3}(2.65 \mathrm{~g}, 7.50 \mathrm{mmol}, 1.5$ equiv.), benzyl alcohol (1.04 mL, $10.0 \mathrm{mmol}, 2.0$ equiv.) and 1-bromo-2-methyl-1-propene $(510 \mu \mathrm{L}$, $5.00 \mathrm{mmol}, 1.0$ equiv.). The reaction mixture was stirred at $80^{\circ} \mathrm{C}$ for $48 \mathrm{~h}$. The reaction mixture was quenched by addition of dist. $\mathrm{H}_{2} \mathrm{O}$ and diluted with $\mathrm{Et}_{2} \mathrm{O}$. The phases were separated and the aqueous phase was extracted with $\mathrm{Et}_{2} \mathrm{O}$. The combined organic phases were washed with brine, dried over $\mathrm{MgSO}_{4}$ and concentrated under reduced pressure. Purification by column chromatography on silica gel (pentane/ $\mathrm{Et}_{2} \mathrm{O}=97 / 3$ ) gave the product as a yellow oil (485 mg, $2.99 \mathrm{mmol}, 60 \%)$.

${ }^{1} \mathrm{H}$ NMR $\left(400 \mathrm{MHz}_{\mathrm{CDCl}}\right.$ ): $\delta 7.39-7.23(\mathrm{~m}, 5 \mathrm{H}), 5.92(\mathrm{~m}, 1 \mathrm{H}), 4.74(\mathrm{~s}, 2 \mathrm{H}), 1.67(\mathrm{~d}, J=1.4$ $\mathrm{Hz}, 3 \mathrm{H}), 1.55(\mathrm{~d}, \mathrm{~J}=1.5 \mathrm{~Hz}, 3 \mathrm{H})$;

${ }^{13} \mathrm{C}$ NMR $\left(101 \mathrm{MHz}, \mathrm{CDCl}_{3}\right): \delta$ 139.9, 138.2, 128.5, 127.8, 127.4, 111.3, 73.4, 19.7, 15.2;

GC MS (EI): 91.1 (100), 65.0 (13), 39.1 (14);

$\mathbf{R}_{\mathbf{f}}($ pentane/Et2O = 97/3): 0.58 .

\section{1-Methoxy-4-((2-methylprop-1-en-1-yl)oxy)benzene}

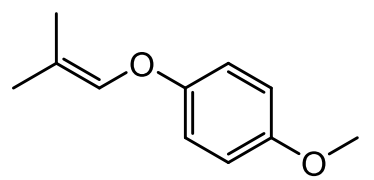

Prepared following two modified literature procedures. ${ }^{78}$ 4-Methoxyphenol (620 mg, $5.00 \mathrm{mmol}, 1.0$ equiv.) and $\mathrm{K}_{2} \mathrm{CO}_{3}(1.38 \mathrm{~g}, 10.0 \mathrm{mmol}$, 2.0 equiv.) were suspended in DMF (25 mL). 3-Bromo-2-methylprop-1ene ( $660 \mu \mathrm{L}, 6.50 \mathrm{mmol}, 1.3$ equiv.) was slowly added and the reaction mixture was stirred at $70{ }^{\circ} \mathrm{C}$ overnight. The reaction mixture was quenched by addition of dist. $\mathrm{H}_{2} \mathrm{O}$ and diluted with $\mathrm{Et}_{2} \mathrm{O}$. The phases were separated and the aqueous phase was extracted with Et $2 \mathrm{O}$. The combined organic phases were washed with brine, dried over $\mathrm{MgSO}_{4}$ and filtered over a short silica plug. After concentration under reduced pressure, the crude allyl ether was added 
to a mixture of $\mathrm{RuCl}_{2}\left(\mathrm{PPh}_{3}\right)_{3}(2 \mathrm{~mol} \%)$ and $\mathrm{LiAlH}_{4}(10 \mathrm{~mol} \%)$ in THF $(5 \mathrm{~mL})$ and stirred at $80{ }^{\circ} \mathrm{C}$ overnight. The reaction mixture was quenched by addition of dist. $\mathrm{H}_{2} \mathrm{O}$ and diluted with $\mathrm{Et}_{2} \mathrm{O}$. The phases were separated and the aqueous phase was extracted with $\mathrm{Et}_{2} \mathrm{O}$. The combined organic phases were washed with brine, dried over $\mathrm{MgSO}_{4}$ and concentrated under reduced pressure. Purification by column chromatography on silica gel (pentane/ $\mathrm{E}_{2} \mathrm{O}=97 / 3$ ) gave the product as a colorless oil ( $575 \mathrm{mg}, 3.23 \mathrm{mmol}, 65 \%$ over two steps).

${ }^{1} \mathrm{H}$ NMR $\left(400 \mathrm{MHz}, \mathrm{CDCl}_{3}\right): \delta 6.94-6.88(\mathrm{~m}, 2 \mathrm{H}), 6.86-6.79(\mathrm{~m}, 2 \mathrm{H}), 6.14(\mathrm{p}, J=1.5 \mathrm{~Hz}, 1 \mathrm{H})$, $3.78(\mathrm{~s}, 3 \mathrm{H}), 1.74-1.71(\mathrm{~m}, 3 \mathrm{H}), 1.68(\mathrm{~d}, J=1.5 \mathrm{~Hz}, 3 \mathrm{H})$;

${ }^{13} \mathrm{C}$ NMR $\left(101 \mathrm{MHz}, \mathrm{CDCl}_{3}\right): \delta 154.8,152.1,136.3,117.0,116.6,114.7,55.8,19.6,15.3 ;$

GC MS (EI): 178.1(100), 124.1(59), 109.1(81), 55.1(19);

$\mathbf{R}_{\mathbf{f}}($ pentane/EtOAc $=97 / 3): 0.67$

\section{Tert-butyldiphenyl(prop-1-en-1-yloxy)silane}

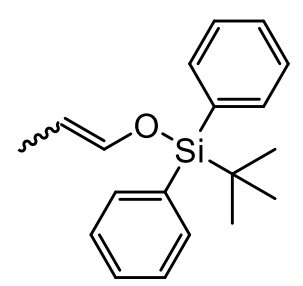

Prepared following a modified literature procedure. ${ }^{3}$ Tertbutyl(chloro)diphenylsilane ( $1.43 \mathrm{~mL}, 24.0 \mathrm{mmol}, 1.2$ equiv.) was added to a stirred solution of 1,8-diazabicyclo[5.4.0]undec-7-en $(4.78 \mathrm{~mL}, 32.0 \mathrm{mmol}$, 1.6 equiv.) in $\mathrm{MeCN}(30 \mathrm{~mL})$ at $0^{\circ} \mathrm{C}$. Propionaldehyde (1.43 mL, $20.0 \mathrm{mmol}$, 1.0 equiv.) was added dropwise at $0^{\circ} \mathrm{C}$, the reaction mixture was allowed to warm to room temperature and stirred overnight. The reaction mixture was quenched with dist. $\mathrm{H}_{2} \mathrm{O}$, the phases were separated and the aqueous phase was extracted with $\mathrm{E}_{2} \mathrm{O}$. The combined organic phases were dried over $\mathrm{MgSO}_{4}$, filtered over a silica plug and concentrated under reduced pressure. The product was obtained as a colorless oil $(3.58 \mathrm{~g}, 12.1 \mathrm{mmol}, 60 \%)$ in an $E / Z$-ratio of $60 / 40$.

${ }^{1} \mathrm{H}$ NMR $\left(400 \mathrm{MHz}, \mathrm{CDCl}_{3}\right) \delta 7.69(\mathrm{~m}, 4 \mathrm{H}), 7.47-7.36(\mathrm{~m}, 6 \mathrm{H}), 6.25(\mathrm{dq}, J=11.8,1.7 \mathrm{~Hz}, 0.6 \mathrm{H})$, $6.21(\mathrm{dq}, J=5.6,1.7 \mathrm{~Hz}, 0.4 \mathrm{H}), 5.12(\mathrm{dq}, J=11.8,6.8 \mathrm{~Hz}, 0.6 \mathrm{H}), 4.53(\mathrm{qd}$, 
$J=6.8,5.6 \mathrm{~Hz}, 0.4 \mathrm{H}), 1.73(\mathrm{dd}, J=6.8,1.7 \mathrm{~Hz}, 1.2 \mathrm{H}), 1.47(\mathrm{dd}, J=6.8,1.7 \mathrm{~Hz}, 1.8 \mathrm{H}), 1.10(\mathrm{~s}$, $3.7 \mathrm{H}), 1.07(\mathrm{~s}, 5.3 \mathrm{H})$;

${ }^{13} \mathrm{C}$ NMR $\left(101 \mathrm{MHz}, \mathrm{CDCl}_{3}\right) \delta 140.8,139.7,135.6,135.5,133.3,133.2,130.0,129.9,127.9$, $127.9,106.2,104.8,26.7,19.5,19.4,12.3,9.3$;

HRMS $\left(E S I^{+}\right):[\mathrm{M}+\mathrm{Na}]^{+} 319.1489$, found 319.1489 ;

$\mathbf{R}_{\mathbf{f}}($ pentane/Et $2 \mathrm{O}=98 / 2): 0.54$.

\section{Dodecyl(2-methylprop-1-en-1-yl)sulfane}<smiles>CCCCSC=C(C)C</smiles>

Prepared following a modified literature procedure. ${ }^{9} \mathrm{Pd}_{2}(\mathrm{dba})_{3}$ (137 $\mathrm{mg}$, $150 \mu \mathrm{mol}, 3.0 \mathrm{~mol} \%$ ) and Xantphos (174 mg, $300 \mu \mathrm{mol}, 6.0 \mathrm{~mol} \%$ ) were dissolved in 1,4-dioxane $(25 \mathrm{~mL})$, before diisopropylethylamine $(1.50 \mathrm{~mL}, 9.00 \mathrm{mmol}$, 1.8 equiv.), dodecanethiol (1.20 mL, $5.00 \mathrm{mmol}, 1.0$ equiv.) and 1-bromo-2-methylprop-1-ene $(640 \mu \mathrm{L}, 6.25 \mathrm{mmol}, 1.25$ equiv.) were added dropwise. The reaction mixture was heated to $110^{\circ} \mathrm{C}$ and stirred overnight. After quenching with dist. water, the phases were separated and the aqueous phase was extracted with $\mathrm{Et}_{2} \mathrm{O}$, washed with dist. water and brine, dried over $\mathrm{MgSO}_{4}$ and concentrated under reduced pressure. Purification by column chromatography on silica gel (pentane/Et $2 \mathrm{O}=99 / 1$ ) gave the product as a yellow oil (990 mg, $3.86 \mathrm{mmol}, 77 \%$ ).

'H NMR $\left(400 \mathrm{MHz}, \mathrm{CDCl}_{3}\right) \delta 5.60(\mathrm{~m}, 1 \mathrm{H}), 2.62-2.58(\mathrm{~m}, 2 \mathrm{H}), 1.77(\mathrm{~d}, J=1.2 \mathrm{~Hz}, 3 \mathrm{H}), 1.74$ (d, $J=1.2 \mathrm{~Hz}, 3 \mathrm{H}), 1.63-1.55(\mathrm{~m}, 2 \mathrm{H}), 1.41-1.33(\mathrm{~m}, 2 \mathrm{H}), 1.26(\mathrm{~d}, J=5.1 \mathrm{~Hz}, 16 \mathrm{H}), 0.90-$ $0.85(\mathrm{~m}, 3 \mathrm{H})$;

${ }^{13} \mathrm{C}$ NMR $\left(75 \mathrm{MHz}, \mathrm{CDCl}_{3}\right) \delta 133.9,118.4,34.2,32.1,30.4,29.8,29.8,29.7,29.7,29.5,29.4$, 28.8, 25.4, 22.8, 19.7, 14.3;

GC MS (EI): 256.2 (85), 88.0 (100), 57.1 (23), 55.1 (38), 43.1 (36), 41.0 (37);

$\mathbf{R}_{\mathbf{f}}($ pentane/Et $2 \mathrm{O}=99 / 1): 0.91$. 
<smiles>[M]C1CCC2(C)C(=O)CCC23CCC(OC(=O)c2cccc(C=O)c2)CC1CC3</smiles>

Prepared following a modified literature procedure. ${ }^{10} 3$ Carboxybenzaldehyde (225 mg, $1.50 \mathrm{mmol}, 1.0$ equiv.) and $\mathrm{N}, \mathrm{N}$-dicyclohexylcarbodiimide $\quad(464 \mathrm{mg}, \quad 2.25 \mathrm{mmol}$, 1.5 equiv.) were dissolved in $\mathrm{CH}_{2} \mathrm{Cl}_{2}(11 \mathrm{~mL})$. Subsequently 4-(dimethylamino)-pyridine $\quad(27.4 \mathrm{mg}, \quad 0.225 \mathrm{mmol}$, $15 \mathrm{~mol} \%$ ) and trans-androsterone (225 mg, $1.50 \mathrm{mmol}, 1.0$ equiv.) were added and the reaction mixture was stirred at room temperature overnight. The reaction mixture was filtered over celite and washed with $\mathrm{CH}_{2} \mathrm{Cl}_{2}$. The filtrate was concentrated under reduced pressure. Purification by column chromatography on silica gel (pentane/EtOAc $=70 / 30$ ) gave the product as a colorless solid (496 mg, $1.17 \mathrm{mmol}, 78 \%$ ).

${ }^{1} \mathrm{H}$ NMR $\left(500 \mathrm{MHz}, \mathrm{CDCl}_{3}\right) \delta 10.08(\mathrm{~s}, 1 \mathrm{H}), 8.51$ (t, $\left.J=1.7 \mathrm{~Hz}, 1 \mathrm{H}\right), 8.29$ (dt, $J=7.7,1.5 \mathrm{~Hz}$, $1 \mathrm{H}$ ), 8.07 (dt, $J=7.7,1.5 \mathrm{~Hz}, 1 \mathrm{H}$ ), $7.62(\mathrm{t}, J=7.7 \mathrm{~Hz}, 1 \mathrm{H}$ ), 4.99 (tt, $J=11.4,4.9 \mathrm{~Hz}, 1 \mathrm{H}), 2.44$ (ddd, $J=19.3,8.9,1.1 \mathrm{~Hz}, 1 \mathrm{H}$ ), $2.12-1.89(\mathrm{~m}, 3 \mathrm{H}), 1.84-1.74(\mathrm{~m}, 4 \mathrm{H}), 1.68$ (ddd, $J=13.6$, 4.6, 2.4 Hz, 2H), $1.62-1.47(\mathrm{~m}, 2 \mathrm{H}), 1.42-1.22(\mathrm{~m}, 7 \mathrm{H}), 1.13(\mathrm{td}, J=13.6,3.9 \mathrm{~Hz}, 1 \mathrm{H}), 1.01$ (qd, $J=12.2,4.9 \mathrm{~Hz}, 1 \mathrm{H}$ ), 0.91 (s, 3H), $0.87(\mathrm{~s}, 3 \mathrm{H}), 0.76$ (ddd, $J=12.3,10.5,4.0 \mathrm{~Hz}, 1 \mathrm{H}$ );

${ }^{13} \mathrm{C}$ NMR $\left(126 \mathrm{MHz}, \mathrm{CDCl}_{3}\right) \delta 221.3,191.6,165.1,136.6,135.3,133.2,132.1,131.2,129.3$, $74.9,54.5,51.5,47.9,44.9,36.9,36.0,35.9,35.2,34.2,31.7,31.0,28.4,27.6,21.9,20.6,14.0$, 12.4

HRMS $\left(\mathrm{ESI}^{+}\right):[\mathrm{M}+\mathrm{Na}]^{+}$445.2349, found 445.2351 ;

$\mathbf{R}_{\mathbf{f}}\left(\right.$ pentane $\left./ \mathrm{Et}_{2} \mathrm{O}=70 / 30\right): 0.47$.

(But-2-en-2-yloxy)(tert-butyl)dimethylsilane

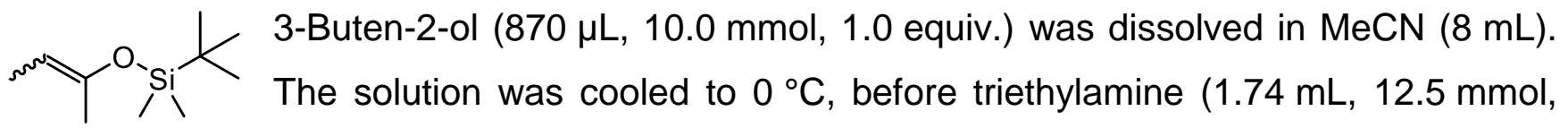


1.25 equiv.) and tert-butyldimethylsilyl chloride (1.88 g, $12.5 \mathrm{mmol}, 1.25$ equiv.) were added. The reaction mixture was allowed to warm to room temperature and stirred overnight. The reaction mixture was quenched with dist. $\mathrm{H}_{2} \mathrm{O}$, the phases were separated and the aqueous phase was extracted with $\mathrm{Et}_{2} \mathrm{O}$. The combined organic phases were dried over $\mathrm{MgSO}_{4}$, filtered over a silica plug and concentrated under reduced pressure to give the crude protected allylic alcohol. $\mathrm{RuCl}_{2}\left(\mathrm{PPh}_{3}\right)_{3}(1.0 \mathrm{~mol} \%)$ and $\mathrm{LiAlH}_{4}(5.0 \mathrm{~mol} \%)$ were dissolved in THF $(5 \mathrm{~mL})$ at room temperature. After stirring for $1 \mathrm{~h}$, a solution of the crude protected allylic alcohol dissolved in THF $(5 \mathrm{~mL})$ was added dropwise and the reaction was stirred at $80^{\circ} \mathrm{C}$ overnight. The reaction mixture was quenched with dist. $\mathrm{H}_{2} \mathrm{O}$, the phases were separated and the aqueous phase was extracted with $\mathrm{Et}_{2} \mathrm{O}$. The combined organic phases were dried over $\mathrm{MgSO}_{4}$ and concentrated under reduced pressure. Purification by column chromatography on silica gel (pentane) gave the product as a colorless oil (160 $\mathrm{mg}, 0.930 \mathrm{mmol}, 9 \%$ over two steps) in an E/Z-ratio of 70/30.

${ }^{1} \mathrm{H}$ NMR $\left(400 \mathrm{MHz}, \mathrm{CDCl}_{3}\right) \delta 4.70-4.63(\mathrm{~m}, 0.3 \mathrm{H}), 4.46(\mathrm{~m}, 0.7 \mathrm{H}), 1.76(\mathrm{~m}, 2.1 \mathrm{H}), 1.72(\mathrm{~m}$, $0.9 \mathrm{H}), 1.51(\mathrm{~m}, 3 \mathrm{H}), 0.96(\mathrm{~s}, 5.7 \mathrm{H}), 0.92(\mathrm{~s}, 3.3 \mathrm{H}), 0.14(\mathrm{~s}, 4.2 \mathrm{H}), 0.11(\mathrm{~s}, 1.8 \mathrm{H})$;

${ }^{13} \mathrm{C}$ NMR $\left(101 \mathrm{MHz}, \mathrm{CDCl}_{3}\right) \delta 148.4,147.6,102.5,102.1,26.0,25.9,22.9,18.4,18.1,12.2$, $11.8,10.8,-3.7,-4.3$

GC MS (EI): 41.1 (10), 45.0 (11), 59.1 (11), 73.0 (15), 75.0 (100), 129.1 (53);

$\mathbf{R}_{\mathbf{f}}\left(\right.$ pentane $\left./ \mathrm{Et}_{2} \mathrm{O}=99 / 1\right): 0.89$.

\section{2,2-Dimethylcyclopropane-1-carbaldehyde}

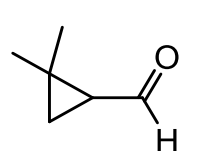

A solution of 2,2-dimethylcyclopropanecarboxylic acid $(900 \mu \mathrm{L}, 8.66 \mathrm{mmol}$, 1.0 equiv.) in $\mathrm{Et}_{2} \mathrm{O}(4.7 \mathrm{~mL}$ ) was slowly added dropwise to a stirring suspension of $\mathrm{LiAlH}_{4}(657 \mathrm{mg}, 17.3 \mathrm{mmol}, 2.0$ equiv. $)$ in $\mathrm{Et}_{2} \mathrm{O}(23 \mathrm{~mL})$ at $0{ }^{\circ} \mathrm{C}$. The reaction mixture was allowed to warm to room temperature and stirred overnight. The reaction mixture was diluted with $\mathrm{Et}_{2} \mathrm{O}$ and carefully quenched with $\mathrm{MeOH}$ and dist. $\mathrm{H}_{2} \mathrm{O}$. The organic phase was separated and filtered over a short silica pad to give the crude alcohol as a colorless oil (611 mg, 
$6.11 \mathrm{mmol}, 71 \%) \cdot \mathrm{CH}_{2} \mathrm{Cl}_{2}(20 \mathrm{~mL})$ and pyridinium chlorochromate $(1.32 \mathrm{~g}, 6.11 \mathrm{mmol}$, 1.0 equiv.) were added and stirred overnight. The reaction was quenched with dist. $\mathrm{H}_{2} \mathrm{O}$, diluted with $\mathrm{Et}_{2} \mathrm{O}$ and transferred to a separation funnel. The remaining black residue was dissolved by addition of $\mathrm{Et}_{2} \mathrm{O}$ and $\mathrm{NaOH}_{\text {(aq.) }}$ and added to the separation funnel as well. The phases were separated and the aqueous phase was extracted with $\mathrm{E}_{2} \mathrm{O}$. The combined organic phases were dried over $\mathrm{MgSO}_{4}$, filtered and carefully concentrated under reduced pressure. Purification by column chromatography on silica gel (pentane $/ \mathrm{Et}_{2} \mathrm{O}=97 / 3$ ) gave the highly volatile aldehyde as a colorless oil $(68.3 \mathrm{mg}, 0.696 \mathrm{mmol}, 8 \%$ over two steps).

${ }^{1} \mathrm{H}$ NMR $\left(400 \mathrm{MHz}, \mathrm{CD}_{2} \mathrm{Cl}_{2}\right) \delta 9.30(\mathrm{~d}, J=5.5 \mathrm{~Hz}, 1 \mathrm{H}), 1.67(\mathrm{dt}, J=8.0,5.3 \mathrm{~Hz}, 1 \mathrm{H}), 1.31(\mathrm{t}, J$ $=4.9 \mathrm{~Hz}, 1 \mathrm{H}), 1.26(\mathrm{~s}, 3 \mathrm{H}), 1.18(\mathrm{~s}, 3 \mathrm{H}), 1.06(\mathrm{dd}, J=8.0,4.5 \mathrm{~Hz}, 1 \mathrm{H})$;

${ }^{13} \mathrm{C}$ NMR $\left(101 \mathrm{MHz}, \mathrm{CD}_{2} \mathrm{Cl}_{2}\right) \delta 201.8,37.1,27.2,26.3,23.6,20.3$;

GC MS (EI): 82.1 (18), 69.1 (31), 67.1 (74), 41.1 (100), 39.1 (54);

$\mathbf{R}_{\mathbf{f}}\left(\right.$ pentane $\left./ \mathrm{Et}_{2} \mathrm{O}=97 / 3\right): 0.48$.

\section{General Procedures for the Synthesis of Mono-Protected 1,2-Diols}

\section{General Procedure 1:}

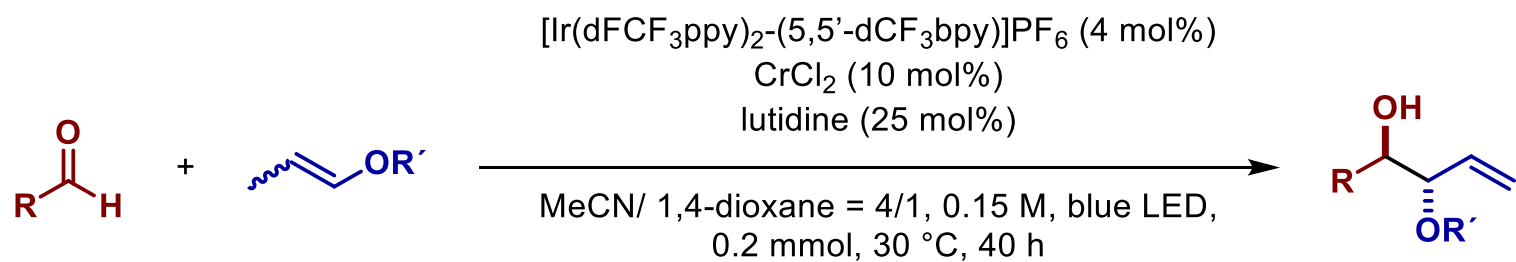

To an oven-dried $10 \mathrm{~mL}$ reaction tube was added $\left[\operatorname{Ir}\left(\mathrm{dFCF}_{3} \mathrm{ppy}\right)_{2}-\left(5,5^{\prime}-\mathrm{dCF}_{3} \mathrm{bpy}\right) \mathrm{PF}_{6}(9.2 \mathrm{mg}\right.$, $8 \mu \mathrm{mol}, 4 \mathrm{~mol} \%)$ and the aldehyde $(0.2 \mathrm{mmol}, 1.0$ equiv., if solid). The tube was transferred to an argon-filled glovebox and $\mathrm{CrCl}_{2}(2.5 \mathrm{mg}, 0.02 \mathrm{mmol}, 10 \mathrm{~mol} \%)$ was added. After sealing and removing the tube from the glovebox, under argon were added MeCN (1.07 mL), 1,4-dioxane ( $0.27 \mathrm{~mL}), 2,6$-lutidine $(5.8 \mu \mathrm{L}, 0.05 \mathrm{mmol}, 25 \mathrm{~mol} \%)$, the allyl substrate (3.0 equiv.) and the aldehyde ( $0.2 \mathrm{mmol}, 1.0$ equiv., if liquid). The reaction mixture was carefully degassed by three freeze/pump/thaw cycles and irradiated with blue LEDs for $40 \mathrm{~h}$. The reaction mixture was 
quenched with an aqueous solution of Rochelle's salt and the phases were separated. The aqueous phase was extracted with EtOAc and the combined organic phases were washed with dist. water, dried over $\mathrm{MgSO}_{4}$, filtered over a short silica plug and concentrated under reduced pressure. The crude residue was dissolved in $\mathrm{CD}_{2} \mathrm{Cl}_{2}$ and the $\mathrm{dr}$ was determined by ${ }^{1} \mathrm{H} \mathrm{NMR}$. The crude reaction mixture was concentrated under reduced pressure and purification by column chromatography on silica gel afforded the title compounds as separate diastereomers.

\section{General Procedure 2:}

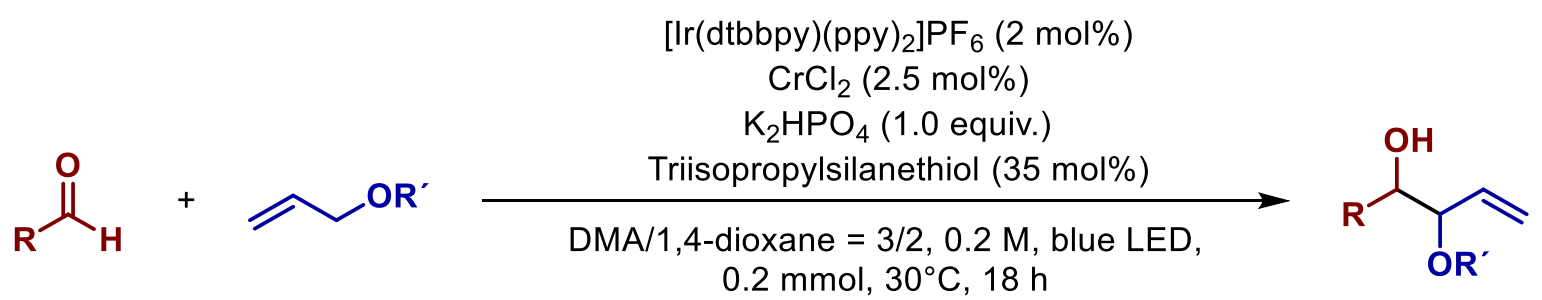

To an oven-dried $10 \mathrm{~mL}$ reaction tube was added [Ir(dtbbpy)(ppy)2)]PF6 (3.7 mg, $0.004 \mathrm{mmol}$, $2 \mathrm{~mol} \%$ ) and the aldehyde ( $0.2 \mathrm{mmol}, 1.0$ equiv., if solid). The tube was transferred to an argonfilled glovebox and $\mathrm{CrCl}_{2}(0.6 \mathrm{mg}, 5 \mu \mathrm{mol}, 2.5 \mathrm{~mol} \%)$ and potassium phosphate dibasic (34.8 $\mathrm{mg}, 0.2 \mathrm{mmol}, 1.0$ equiv.) were added. After sealing and removing the tube from the glovebox, under argon were added DMA $(0.72 \mathrm{~mL}), 1,4$-dioxane $(0.48 \mathrm{~mL})$, the allyl substrate (3.0 equiv.), triisopropylsilanethiol ( $15 \mu \mathrm{L}, 0.07 \mathrm{mmol}, 35 \mathrm{~mol} \%)$ and the aldehyde $(0.2 \mathrm{mmol}$, 1.0 equiv., if liquid). The reaction mixture was carefully degassed by three freeze/pump/thaw cycles and irradiated with blue LEDs for $18 \mathrm{~h}$. The reaction mixture was quenched with an aqueous solution of Rochelle's salt and the phases were separated. The aqueous phase was extracted with $\mathrm{EtOAc} / \mathrm{Et}_{2} \mathrm{O}$ and the combined organic phases were washed with dist. water, dried over $\mathrm{MgSO}_{4}$, filtered over a short silica plug and concentrated under reduced pressure. The crude residue was dissolved in $\mathrm{CD}_{2} \mathrm{Cl}_{2}$ and the $\mathrm{dr}$ was determined by ${ }^{1} \mathrm{H}$ NMR. The crude reaction mixture was concentrated under reduced pressure and purification by column chromatography on silica gel afforded the title compounds as separate diastereomers. 


\section{Synthesis of 1,2- Diols}

\section{2-Methyl-3-((triisopropylsilyl)oxy)tridec-1-en-4-ol (2a)}

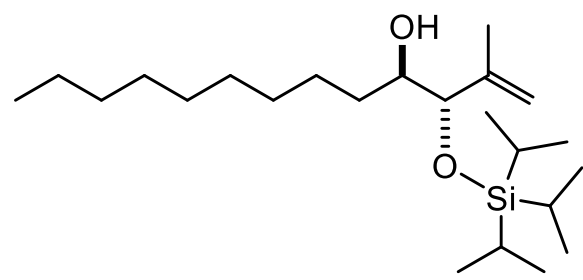

The title compound was synthesized according to General Procedure 1 from decanal ( $31.3 \mathrm{mg}, 0.2 \mathrm{mmol}, 1.0$ equiv) and

$0.6 \mathrm{mmol}, 3.0$ equiv.) with a photocatalyst loading of $4 \mathrm{~mol} \%$.

Purification by column chromatography on silica gel (pentane/Et $\mathrm{t}_{2} \mathrm{O}=98 / 2$ ) gave the two separate diastereomers as colorless oils (combined: $66.8 \mathrm{mg}, 0.174 \mathrm{mmol}, 87 \%$, anti:syn $=$ 78:22).

\section{Anti:}

${ }^{1} \mathrm{H}$ NMR $\left(300 \mathrm{MHz}, \mathrm{CD}_{2} \mathrm{Cl}_{2}\right): \delta 4.93(\mathrm{~m}, 1 \mathrm{H}), 4.86(\mathrm{~m}, 1 \mathrm{H}), 4.09(\mathrm{~d}, J=4.6 \mathrm{~Hz}, 1 \mathrm{H}), 3.72-3.57$ (m, 1H), $2.24(\mathrm{~d}, J=2.5 \mathrm{~Hz}, 1 \mathrm{H}), 1.77(\mathrm{~s}, 3 \mathrm{H}), 1.53-1.37(\mathrm{~m}, 3 \mathrm{H}), 1.27(\mathrm{~m}, 16 \mathrm{H}), 1.07(\mathrm{~s}, 18 \mathrm{H})$, 0.88 (t, $J=6.7 \mathrm{~Hz}, 3 \mathrm{H}$ );

${ }^{13} \mathrm{C}$ NMR $\left(75 \mathrm{MHz}, \mathrm{CD}_{2} \mathrm{Cl}_{2}\right): \delta$ 145.6, 114.3, 80.4, 74.5, 32.9, 32.5, 30.3, 30.2, 29.9, 26.7, 23.3, 19.1, 18.4, 18.4, 14.4, 12.9;

HRMS (ESI+): [M+Na]+ 407.3316, found 407.3322;

$\mathbf{R}_{\mathbf{f}}($ pentane/EtOAc $=98 / 2): 0.63$

\section{5-Methyl-4-((triisopropylsilyl)oxy)hexa-1,5-dien-3-ol (2b)}

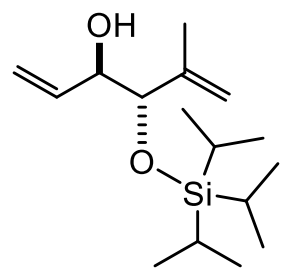

The title compound was synthesized according to General Procedure 1 from acrolein (techn. $90 \%, 14.8 \mu \mathrm{L}, 0.2 \mathrm{mmol}, 1.0$ equiv.) and triisopropyl((2methylprop-1-en-1-yl)oxy)silane (137 mg, $0.6 \mathrm{mmol}, 3.0$ equiv.) with a photocatalyst loading of $4 \mathrm{~mol} \%$. Purification by column chromatography on silica gel (pentane/Et2O $=99 / 5$ ) gave the separate diastereomers as colorless oils (combined: $43.2 \mathrm{mg}, 0.152 \mathrm{mmol}, 76 \%$, anti:syn $=77: 23$ ). 


\section{Anti:}

${ }^{1} \mathrm{H}$ NMR $\left(400 \mathrm{MHz}, \mathrm{CD}_{2} \mathrm{Cl}_{2}\right): \delta 5.85(\mathrm{~m}, 1 \mathrm{H}), 5.28(\mathrm{~m}, 1 \mathrm{H}), 5.15(\mathrm{~m}, 1 \mathrm{H}), 4.94-4.90(\mathrm{~m}, 2 \mathrm{H})$, $4.19-4.13(\mathrm{~m}, 2 \mathrm{H}), 2.28(\mathrm{~d}, J=3.0 \mathrm{~Hz}, 1 \mathrm{H}), 1.76(\mathrm{~m}, 3 \mathrm{H}), 1.18-1.09(\mathrm{~m}, 3 \mathrm{H}), 1.09-1.04(\mathrm{~m}$, $18 \mathrm{H})$;

${ }^{13} \mathrm{C}$ NMR $\left(101 \mathrm{MHz}, \mathrm{CD}_{2} \mathrm{Cl}_{2}\right): \delta$ 145.3, 137.7, 116.2, 114.5, 80.4, 75.7, 19.1, 18.4, 18.4, 13.0;

HRMS $\left(\mathrm{ESI}{ }^{+}\right):[\mathrm{M}+\mathrm{Na}]^{+}$307.2064, found 307.2060;

$\mathbf{R}_{\mathbf{f}}($ pentane/EtOAc $=95 / 5): 0.47$

\section{2,5-Dimethyl-4-((triisopropylsilyl)oxy)hex-5-en-3-ol (2c)}

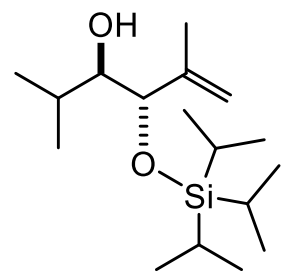

The title compound was synthesized according to General Procedure 1 from isobutyraldehyde (14.4 $\mathrm{mg}, \quad 0.2 \mathrm{mmol}, \quad 1.0$ equiv.) and triisopropyl((2methylprop-1-en-1-yl)oxy)silane (137 mg, $0.6 \mathrm{mmol}, 3.0$ equiv.) with a photocatalyst loading of $4 \mathrm{~mol} \%$. Purification by column chromatography on silica gel (pentane/ $\mathrm{Et}_{2} \mathrm{O}=98 / 2$ ) gave the two separate diastereomers as colorless oils (combined: $51.1 \mathrm{mg}, 0.170 \mathrm{mmol}, 85 \%$, anti:syn = 80:20).

\section{Anti:}

${ }^{1} \mathrm{H}$ NMR $\left(400 \mathrm{MHz}, \mathrm{CD}_{2} \mathrm{Cl}_{2}\right): \delta 4.93(\mathrm{~m}, 1 \mathrm{H}), 4.89(\mathrm{~m}, 1 \mathrm{H}), 4.27(\mathrm{~d}, J=4.5 \mathrm{~Hz}, 1 \mathrm{H}), 3.38-3.29$ (m, 1H), $2.45(\mathrm{~d}, J=1.7 \mathrm{~Hz}, 1 \mathrm{H}), 1.80(\mathrm{~s}, 3 \mathrm{H}), 1.66-1.56(\mathrm{~m}, 1 \mathrm{H}), 1.10-1.03(\mathrm{~m}, 21 \mathrm{H}), 0.97$ (d, $J=6.6 \mathrm{~Hz}, 3 \mathrm{H}), 0.88(\mathrm{~d}, J=6.7 \mathrm{~Hz}, 3 \mathrm{H})$;

${ }^{13} \mathrm{C}$ NMR $\left(101 \mathrm{MHz}, \mathrm{CD}_{2} \mathrm{Cl}_{2}\right): \delta 145.6,114.7,79.9,78.4,29.8,19.7,19.2,19.0,18.4,12.9$;

HRMS $\left(\mathrm{ESI}{ }^{+}\right):[\mathrm{M}+\mathrm{Na}]^{+}$323.2379, found 323.2375;

$\mathbf{R}_{\mathbf{f}}($ pentane/EtOAc $=98 / 2): 0.59$. 


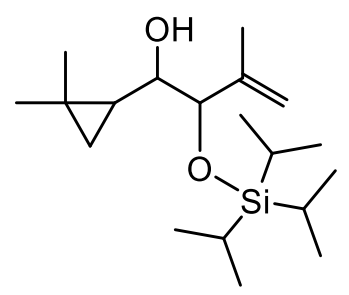

The title compound was synthesized according to General Procedure 1 from 2,2-dimethylcyclopropane-1-carbaldehyde ( $19.6 \mathrm{mg}, 0.2 \mathrm{mmol}, 1.0$ equiv.) and triisopropyl((2-methylprop-1-en-1-yl)oxy)silane (137 mg, $0.6 \mathrm{mmol}$, 3.0 equiv.) with a photocatalyst loading of $4 \mathrm{~mol} \%$. Purification by column chromatography on silica gel (pentane $/ \mathrm{Et}_{2} \mathrm{O}=97 / 3$ ) gave the two separate diastereomers as colorless oils (combined: $45.7 \mathrm{mg}, 0.140 \mathrm{mmol}, 70 \%$, anti:syn $=56: 44$ ).

HRMS $\left(\mathrm{ESI}{ }^{+}\right):[\mathrm{M}+\mathrm{Na}]^{+}$349.2533, found 349.2530;

Syn:

${ }^{1} \mathrm{H}$ NMR $\left(400 \mathrm{MHz}, \mathrm{CD}_{2} \mathrm{Cl}_{2}\right): \delta 5.00(\mathrm{~m}, 1 \mathrm{H}), 4.88(\mathrm{~m}, 1 \mathrm{H}), 4.14(\mathrm{~m}, 1 \mathrm{H}), 3.08$ (ddd, $J=9.7,6.2$, $3.5 \mathrm{~Hz}, 1 \mathrm{H}), 2.47(\mathrm{~d}, J=3.5 \mathrm{~Hz}, 1 \mathrm{H}), 1.74(\mathrm{t}, J=1.2 \mathrm{~Hz}, 3 \mathrm{H}), 1.14(\mathrm{~s}, 3 \mathrm{H}), 1.11-1.06(\mathrm{~m}, 21 \mathrm{H})$, 1.06 (s, 3H), 0.62 (ddd, $J=9.7,8.7,5.4 \mathrm{~Hz}, 1 \mathrm{H}), 0.38$ (dd, $J=8.7,4.6 \mathrm{~Hz}, 1 \mathrm{H}), 0.12(\mathrm{~m}, 1 \mathrm{H})$;

${ }^{13} \mathrm{C}$ NMR $\left(101 \mathrm{MHz}, \mathrm{CD}_{2} \mathrm{Cl}_{2}\right): \delta 146.3,113.8,81.9,76.0,28.3,27.7,20.7,19.5,18.9,18.5,18.5$, 16.1, 13.2;

$\mathbf{R}_{\mathbf{f}}($ pentane/EtOAc $=97 / 3): 0.86$

\section{Anti:}

${ }^{1} \mathrm{H}$ NMR $\left(400 \mathrm{MHz}, \mathrm{CD}_{2} \mathrm{Cl}_{2}\right): \delta 4.97-4.95(\mathrm{~m}, 1 \mathrm{H}), 4.88(\mathrm{~m}, 1 \mathrm{H}), 4.20(\mathrm{~m}, 1 \mathrm{H}), 3.34(\mathrm{~m}, 1 \mathrm{H})$, $2.51(\mathrm{~d}, J=2.1 \mathrm{~Hz}, 1 \mathrm{H}), 1.83(\mathrm{t}, J=1.2 \mathrm{~Hz}, 3 \mathrm{H}), 1.14-1.07(\mathrm{~m}, 3 \mathrm{H}), 1.08-1.06(\mathrm{~m}, 21 \mathrm{H}), 0.98$ (s, 3H), $0.62-0.56(\mathrm{~m}, 1 \mathrm{H}), 0.51$ (dd, $J=8.5,4.2 \mathrm{~Hz}, 1 \mathrm{H}), 0.28(\mathrm{dd}, J=5.4,4.1 \mathrm{~Hz}, 1 \mathrm{H}$ );

${ }^{13} \mathrm{C}$ NMR $\left(101 \mathrm{MHz}, \mathrm{CD}_{2} \mathrm{Cl}_{2}\right): \delta 145.6,114.1,79.9,76.9,27.4,26.6,21.4,19.9,18.9,18.4,18.4$, 16.1, 12.9;

$\mathbf{R}_{\mathbf{f}}($ pentane/EtOAc $=97 / 3): 0.82$ 


\section{3-Methyl-1-phenyl-2-((triisopropylsilyl)oxy)but-3-en-1-ol (2e)}

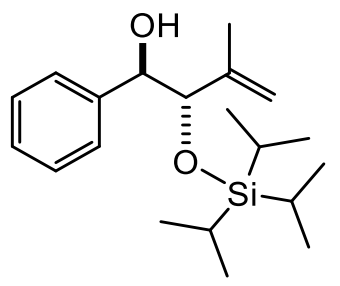

The title compound was synthesized according to General Procedure 1 from benzaldehyde $(20.3 \mu \mathrm{L}, \quad 0.2 \mathrm{mmol}, 1.0$ equiv.) and triisopropyl((2methylprop-1-en-1-yl)oxy)silane (137 mg, $0.6 \mathrm{mmol}, 3.0$ equiv.) with a photocatalyst loading of $4 \mathrm{~mol} \%$. Purification by column chromatography on silica gel (pentane/ $\mathrm{Et}_{2} \mathrm{O}=95 / 5$ ) gave the two diastereomers as colorless oils (combined: $60.2 \mathrm{mg}, 0.180 \mathrm{mmol}, 90 \%$, anti:syn = 78:22).

Anti:

${ }^{1} \mathrm{H}$ NMR $\left(400 \mathrm{MHz}, \mathrm{CD}_{2} \mathrm{Cl}_{2}\right): \delta 7.37-7.21(\mathrm{~m}, 5 \mathrm{H}), 4.85(\mathrm{~m}, 1 \mathrm{H}), 4.79-4.71(\mathrm{~m}, 2 \mathrm{H}), 4.32(\mathrm{~d}$, $J=5.6 \mathrm{~Hz}, 1 \mathrm{H}), 2.58(\mathrm{~d}, J=2.1 \mathrm{~Hz}, 1 \mathrm{H}), 1.66(\mathrm{~m}, 3 \mathrm{H}), 1.10-0.96(\mathrm{~m}, 21 \mathrm{H})$;

${ }^{13} \mathrm{C}$ NMR $\left(101 \mathrm{MHz}, \mathrm{CD}_{2} \mathrm{Cl}_{2}\right): \delta 145.0,141.3,128.4,127.9,127.4,115.2,81.7,76.8,18.9,18.4$, 18.3, 13.0;

HRMS $\left(\mathrm{ESI}^{+}\right):[\mathrm{M}+\mathrm{Na}]^{+}$357.2220, found 357.2222;

$\mathbf{R}_{\mathbf{f}}($ pentane/EtOAc $=95 / 5): 0.45$.

\section{3-methyl-1-(naphthalen-1-yl)-2-((triisopropylsilyl)oxy)but-3-en-1-ol (2f)}

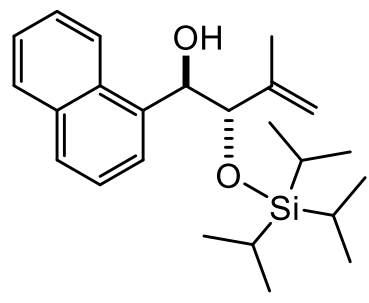

The title compound was synthesized according to General Procedure 1 from 1-naphthaldehyde $(27.1 \mu \mathrm{L}, \quad 0.2 \mathrm{mmol}, \quad 1.0$ equiv. $)$ and triisopropyl((2-methylprop-1-en-1-yl)oxy)silane (137 mg, $0.6 \mathrm{mmol}$, 3.0 equiv.) with a photocatalyst loading of $4 \mathrm{~mol} \%$. Purification by column chromatography on silica gel (pentane/Et $2 \mathrm{O}=95 / 5$ ) gave the two separate diastereomers as colorless oils (combined: $65.4 \mathrm{mg}, 0.170 \mathrm{mmol}, 85 \%$, anti:syn = 72:28).

\section{$\underline{\text { Anti: }}$}

${ }^{1} \mathrm{H}$ NMR $\left(400 \mathrm{MHz}, \mathrm{CD}_{2} \mathrm{Cl}_{2}\right): \delta 8.10$ (dd, $\left.J=8.5,1.5 \mathrm{~Hz}, 1 \mathrm{H}\right), 7.89-7.85(\mathrm{~m}, 1 \mathrm{H}), 7.80-7.71$ $(\mathrm{m}, 2 \mathrm{H}), 7.55-7.44(\mathrm{~m}, 3 \mathrm{H}), 5.58(\mathrm{~d}, J=5.6 \mathrm{~Hz}, 1 \mathrm{H}), 4.78(\mathrm{t}, J=1.7 \mathrm{~Hz}, 1 \mathrm{H}), 4.68-4.63(\mathrm{~m}$, 2H), $2.94(\mathrm{~d}, J=1.5 \mathrm{~Hz}, 1 \mathrm{H}), 1.64(\mathrm{t}, J=1.2 \mathrm{~Hz}, 3 \mathrm{H}), 1.02-0.96(\mathrm{~m}, 21 \mathrm{H})$; 
${ }^{13} \mathrm{C}$ NMR $\left(101 \mathrm{MHz}, \mathrm{CD}_{2} \mathrm{Cl}_{2}\right): \delta 145.1,137.0,134.2,131.7,129.3,128.3,126.3,125.9,125.8$, $124.9,123.9,114.9,80.6,73.7,19.0,18.3,18.3,13.0$;

HRMS $\left(\mathrm{ESI}^{+}\right):[\mathrm{M}+\mathrm{Na}]^{+}$407.2377, found 407.2377;

$\mathbf{R}_{\mathbf{f}}($ pentane/EtOAc $=95 / 5): 0.58$.

\section{1-(4-Fluorophenyl)-3-methyl-2-((triisopropylsilyl)oxy)but-3-en-1-ol (2g)}<smiles>C=C(C)[C@H](O[Si](C(C)C)(C(C)C)C(C)C)C(O)c1ccc(F)cc1</smiles>

The title compound was synthesized according to General Procedure 1 from 4-fluoromethylbenzaldehyde (24.8 mg, $0.2 \mathrm{mmol}, 1.0$ equiv.) and triisopropyl((2-methylprop-1-en-1-yl)oxy)silane $\quad(137 \mathrm{mg}, \quad 0.6 \mathrm{mmol}$, 3.0 equiv.) with a photocatalyst loading of $4 \mathrm{~mol} \%$. Purification by column chromatography on silica gel (pentane/Et2O $=98 / 2$ ) gave the two separate diastereomers as colorless oils (combined: $64.9 \mathrm{mg}, 0.184 \mathrm{mmol}, 92 \%$, anti:syn = 78:22).

\section{Anti:}

${ }^{1} \mathrm{H}$ NMR $\left(400 \mathrm{MHz}, \mathrm{CD}_{2} \mathrm{Cl}_{2}\right): \delta 7.39-7.28(\mathrm{~m}, 2 \mathrm{H}), 7.06-6.95(\mathrm{~m}, 2 \mathrm{H}), 4.88(\mathrm{~m}, 1 \mathrm{H}), 4.78(\mathrm{~m}$, $1 \mathrm{H}), 4.71(\mathrm{~m}, 1 \mathrm{H}), 4.27(\mathrm{~d}, J=5.9 \mathrm{~Hz}, 1 \mathrm{H}), 2.58(\mathrm{~d}, J=2.0 \mathrm{~Hz}, 1 \mathrm{H}), 1.68(\mathrm{~m}, 3 \mathrm{H}), 1.07-0.95$ $(\mathrm{m}, 21 \mathrm{H})$;

${ }^{13} \mathrm{C}$ NMR (101 MHz, $\left.\mathrm{CD}_{2} \mathrm{Cl}_{2}\right): \delta 162.8(\mathrm{~d}, J=244.1 \mathrm{~Hz}), 144.9,129.1(\mathrm{~d}, J=8.1 \mathrm{~Hz}), 115.5$, $115.1(\mathrm{~d}, \mathrm{~J}=21.4 \mathrm{~Hz}), 81.8,81.8,76.2,18.8,18.4,18.3,13.0$;

${ }^{19}$ F NMR (376 MHz, $\left.\mathrm{CD}_{2} \mathrm{Cl}_{2}\right): \delta-116.3$;

HRMS $\left(\mathrm{ESI}^{+}\right):[\mathrm{M}+\mathrm{Na}]^{+}$375.2126, found 375.2126;

$\mathbf{R}_{\mathbf{f}}($ pentane/EtOAc $=98 / 2): 0.45$. 


\section{3-Methyl-1-(4-(4,4,5,5-tetramethyl-1,3,2-dioxaborolan-2-yl)phenyl)-2-}

\section{((triisopropylsilyl)oxy)but-3-en-1-ol (2h)}<smiles>C=C(C)[C@H](O[Si](C(C)C)(C(C)C)C(C)C)C(O)c1ccc(Cc2ccccc2)cc1</smiles>

The title compound was synthesized according to General Procedure 1 from 4-(4,4,5,5-tetramethyl-1,3,2-dioxaborolan-2yl)benzaldehyde (46.4 mg, $0.2 \mathrm{mmol}, 1.0$ equiv.) and triisopropyl((2methylprop-1-en-1-yl)oxy)silane ( $137 \mathrm{mg}, 0.6 \mathrm{mmol}, 3.0$ equiv.) with a photocatalyst loading of $4 \mathrm{~mol} \%$. Purification by column chromatography on silica gel (pentane/ $\mathrm{Et}_{2} \mathrm{O}=97 / 3$ ) gave the two separate diastereomers as colorless oils (combined: $71.8 \mathrm{mg}, 0.156 \mathrm{mmol}, 78 \%$, anti:syn = 78:22).

$\underline{\text { Anti: }}$

${ }^{1} \mathrm{H}$ NMR $\left(400 \mathrm{MHz}, \mathrm{CD}_{2} \mathrm{Cl}_{2}\right)$ : $\delta 7.69(\mathrm{~m}, 2 \mathrm{H}), 7.34(\mathrm{~m}, 2 \mathrm{H}), 4.83-4.78(\mathrm{~m}, 2 \mathrm{H}), 4.72-4.70(\mathrm{~m}$, $1 \mathrm{H}), 4.36(\mathrm{~d}, J=5.2,0.7 \mathrm{~Hz}, 1 \mathrm{H}), 2.68(\mathrm{~d}, J=2.1 \mathrm{~Hz}, 1 \mathrm{H}), 1.63(\mathrm{~s}, 3 \mathrm{H}), 1.33(\mathrm{~s}, 12 \mathrm{H}), 1.10-$ $0.98(\mathrm{~m}, 21 \mathrm{H})$;

${ }^{13} \mathrm{C}$ NMR $\left(101 \mathrm{MHz}, \mathrm{CD}_{2} \mathrm{Cl}_{2}\right): \delta$ 144.7, 144.3, 134.8, 127.0, 126.6, 115.1, 84.3, 81.4, 77.1, 25.3, 25.2, 19.2, 18.4, 18.3, 13.0;

${ }^{11}$ B NMR (128 MHz, $\left.\mathrm{CD}_{2} \mathrm{Cl}_{2}\right): \delta-32.5$;

HRMS $\left(\mathrm{ESI}{ }^{+}\right):[\mathrm{M}+\mathrm{Na}]^{+}$483.3072, found 483.3073;

$\mathbf{R}_{\mathbf{f}}($ pentane/EtOAc $=98 / 2): 0.36$.

3-Methyl-1-(4-(trifluoromethyl)phenyl)-2-((triisopropylsilyl)oxy)but-3-en-1-ol (2i)<smiles>C=C(C)[C@H](O[Si](C(C)C)(C(C)C)C(C)C)C(O)c1ccc(C(F)(F)F)cc1</smiles>
The title compound was synthesized according to General Procedure 1 from 4-trifluoromethylbenzaldehyde ( $34.8 \mathrm{mg}, 0.2 \mathrm{mmol}, 1.0$ equiv.) and triisopropyl((2-methylprop-1-en-1-yl)oxy)silane (137 mg, $0.6 \mathrm{mmol}$, 3.0 equiv.) with a photocatalyst loading of $4 \mathrm{~mol} \%$. Purification by 
column chromatography on silica gel (pentane/Et2O $=98 / 2$ ) gave the two separate diastereomers as colorless oils $(78.1 \mathrm{mg}, 0.194 \mathrm{mmol}, 97 \%$, anti:syn $=81: 19)$.

\section{Anti:}

${ }^{1} \mathrm{H}$ NMR $\left(400 \mathrm{MHz}, \mathrm{CD}_{2} \mathrm{Cl}_{2}\right): \delta 7.58(\mathrm{~d}, J=8.2 \mathrm{~Hz}, 2 \mathrm{H}), 7.50(\mathrm{~d}, J=8.2 \mathrm{~Hz}, 2 \mathrm{H}), 4.89(\mathrm{~m}, 1 \mathrm{H})$, $4.82-4.76(\mathrm{~m}, 2 \mathrm{H}), 4.32(\mathrm{~d}, J=5.8 \mathrm{~Hz}, 1 \mathrm{H}), 2.68(\mathrm{~s}, 1 \mathrm{H}), 1.70(\mathrm{~s}, 3 \mathrm{H}), 1.03-0.97(\mathrm{~m}, 21 \mathrm{H})$;

${ }^{13} \mathrm{C}$ NMR $\left(101 \mathrm{MHz}, \mathrm{CD}_{2} \mathrm{Cl}_{2}\right): \delta$ 145.0, 144.0, 129.3 (q, $\left.J=32.1 \mathrm{~Hz}\right), 127.3,124.7$ (q, $J=3.8$ $\mathrm{Hz}), 124.4(\mathrm{q}, J=271.7 \mathrm{~Hz}), 115.2,81.1,75.7,18.1,17.7(\mathrm{~d}, J=4.3 \mathrm{~Hz}), 12.4$;

$\left.{ }^{19} \mathrm{~F} \mathrm{NMR} \mathrm{(376} \mathrm{MHz,} \mathrm{CD}_{2} \mathrm{Cl}_{2}\right): \delta-62.7 ;$

HRMS $\left(E S I^{+}\right):[\mathrm{M}+\mathrm{Na}]^{+}$425.2094, found 425.2093;

$\mathbf{R}_{\mathbf{f}}($ pentane/EtOAc $=98 / 2): 0.38$.

\section{1-(4-Methoxyphenyl)-3-methyl-2-((triisopropylsilyl)oxy)but-3-en-1-ol (2j)}<smiles>C=C(C)[C@H](O[Si](C(C)C)(C(C)C)C(C)C)C(O)c1ccc(OC)cc1</smiles>

The title compound was synthesized according to General Procedure 1 from 4-methoxybenzaldehyde $(27.2 \mathrm{mg}, 0.2 \mathrm{mmol}, 1.0$ equiv.) and triisopropyl((2-methylprop-1-en-1-yl)oxy)silane $\quad(137 \mathrm{mg}, \quad 0.6 \mathrm{mmol}$, 3.0 equiv.) with a photocatalyst loading of $4 \mathrm{~mol} \%$. Purification by column chromatography on silica gel (pentane/Et2O $=98 / 2$ ) gave the two separate diastereomers as colorless oils (combined: $65.0 \mathrm{mg}, 0.178 \mathrm{mmol}, 89 \%$, anti:syn $=66: 34$ ).

Anti:

${ }^{1} \mathrm{H}$ NMR $\left(400 \mathrm{MHz}, \mathrm{CD}_{2} \mathrm{Cl}_{2}\right): \delta 7.26(\mathrm{~d}, J=8.8 \mathrm{~Hz}, 2 \mathrm{H}), 6.84(\mathrm{~d}, J=8.8 \mathrm{~Hz}, 2 \mathrm{H}), 4.89-4.84(\mathrm{~m}$, $1 \mathrm{H}), 4.79-4.74(\mathrm{~m}, 1 \mathrm{H}), 4.68(\mathrm{~d}, J=5.6 \mathrm{~Hz}, 1 \mathrm{H}), 4.28(\mathrm{~d}, J=5.6 \mathrm{~Hz}, 1 \mathrm{H}), 3.78(\mathrm{~s}, 3 \mathrm{H}), 2.52(\mathrm{~s}$, $1 \mathrm{H}), 1.66(\mathrm{~s}, 3 \mathrm{H}), 1.01(\mathrm{~s}, 21 \mathrm{H})$;

${ }^{13} \mathrm{C}$ NMR $\left(101 \mathrm{MHz}, \mathrm{CD}_{2} \mathrm{Cl}_{2}\right): \delta 145.2,133.4,128.5,115.1,113.8,81.7,76.5,55.8,19.0,18.4$, 18.3, 13.0; 
HRMS $\left(\mathrm{ESI}{ }^{+}\right):[\mathrm{M}+\mathrm{Na}]^{+}$387.2326, found 387.2324;

$\mathbf{R}_{\mathbf{f}}($ pentane/EtOAc $=98 / 2): 0.30$.

\section{1-(2-(Allyloxy)phenyl)-3-methyl-2-((triisopropylsilyl)oxy)but-3-en-1-ol (2k)}<smiles>C=CCOc1ccccc1C(O)C(O[Si](C(C)C)(C(C)C)C(C)C)C(=C)C</smiles>

The diasteromers were not separable via column chromatography.

\section{Anti+Syn-Mix:}

${ }^{1} \mathrm{H}$ NMR $\left(400 \mathrm{MHz}, \mathrm{CD}_{2} \mathrm{Cl}_{2}\right): \delta 7.29$ (ddd, $\left.J=7.7,5.8,1.8 \mathrm{~Hz}, 1 \mathrm{H}\right), 7.11-7.02(\mathrm{~m}, 1 \mathrm{H}), 6.81$ (dtd, $J=10.2,7.5,1.1 \mathrm{~Hz}, 1 \mathrm{H}$ ), 6.70 (dd, $J=8.2,1.0 \mathrm{~Hz}, 1 \mathrm{H}$ ), 5.95 (ddt, $J=17.3,10.7,5.5 \mathrm{~Hz}$, 1H), 5.27 (dp, $J=17.2,1.7 \mathrm{~Hz}, 1 \mathrm{H}$ ), $5.20-5.12(\mathrm{~m}, 1 \mathrm{H}), 5.00(\mathrm{dd}, J=4.9,2.8 \mathrm{~Hz}, 0.6 \mathrm{H}), 4.82$ (dd, $J=6.3,4.5 \mathrm{~Hz}, 0.3 \mathrm{H}), 4.72-4.32(\mathrm{~m}, 5 \mathrm{H}), 2.97(\mathrm{~d}, J=2 . \mathrm{Hz}, 0.6 \mathrm{H}), 2.88(\mathrm{~d}, J=6.3 \mathrm{~Hz}$, $0.3), 1.68(\mathrm{t}, J=1.2 \mathrm{~Hz}, 1.1 \mathrm{H}), 1.47(\mathrm{t}, J=1.2 \mathrm{~Hz}, 1.9 \mathrm{H}), 0.99-0.83(\mathrm{~m}, 21 \mathrm{H})$;

${ }^{13} \mathrm{C}$ NMR (101 MHz, $\left.\mathrm{CD}_{2} \mathrm{Cl}_{2}\right): \delta 156.2,155.9,146.3,145.3,134.1,134.1,131.1,129.5,128.7$, 128.5, 128.3, 128.0, 121.0, 121.0, 118.3, 118.1, 114.0, 113.0, 111.7, 111.5, 80.4, 78.2, 73.3, $71.0,69.6,69.6,19.6,19.1,18.4,18.4,13.2,12.9$;

HRMS $\left(\mathrm{ESI}^{+}\right)$: $[\mathrm{M}+\mathrm{Na}]^{+}$413.2482, found 413.2448;

$\mathbf{R}_{\mathbf{f}}($ pentane/EtOAc $=95 / 5): 0.48$ 


\section{2,3-Dibromo-4-(1-hydroxy-3-methyl-2-((triisopropylsilyl)oxy)but-3-en-1-yl)-6-}

methoxyphenol (2I)<smiles>C=C(C)[C@H](O[Si](C(C)C)(C(C)C)C(C)C)C(O)c1cc(OC)c(O)c(Br)c1Br</smiles>

The title compound was synthesized according to General Procedure 1 from 2,3-dibromo-4-hydroxy-5-methoxybenzaldehyde $\quad(62.0 \mathrm{mg}$, $0.2 \mathrm{mmol}, \quad 1.0$ equiv.) and triisopropyl((2-methylprop-1-en-1yl)oxy)silane (137 mg, $0.6 \mathrm{mmol}, 3.0$ equiv.) with a photocatalyst loading of $4 \mathrm{~mol} \%$. Purification by column chromatography on silica gel (pentane/Et ${ }_{2} \mathrm{O}=85 / 15$ ) gave the two separate diastereomers as colorless solids (combined: $77.5 \mathrm{mg}, 0.144 \mathrm{mmol}, 72 \%$, anti:syn = 75:25).

Anti:

${ }^{1} \mathrm{H}$ NMR $\left(400 \mathrm{MHz}, \mathrm{CD}_{2} \mathrm{Cl}_{2}\right): \delta 7.1(\mathrm{~s}, 1 \mathrm{H}), 6.2(\mathrm{~s}, 1 \mathrm{H}), 5.2(\mathrm{~d}, J=4.6 \mathrm{~Hz}, 1 \mathrm{H}), 4.8-4.7(\mathrm{~m}, 1 \mathrm{H})$, $4.7-4.6(\mathrm{~m}, 2 \mathrm{H}), 3.9(\mathrm{~s}, 3 \mathrm{H}), 3.2(\mathrm{~d}, J=1.3 \mathrm{~Hz}, 1 \mathrm{H}), 1.6(\mathrm{t}, J=1.1 \mathrm{~Hz}, 3 \mathrm{H}), 1.2-1.1(\mathrm{~m}, 21 \mathrm{H})$;

${ }^{13} \mathrm{C}$ NMR (101 MHz, $\left.\mathrm{CD}_{2} \mathrm{Cl}_{2}\right): \delta 146.7,144.3,144.2,133.1,115.8,114.9,111.7,110.4,77.5$, 77.0, 57.0, 19.9, 18.4, 18.3, 12.9;

HRMS (ESI+): $[\mathrm{M}+\mathrm{Na}]^{+} 561.0466$, found 561.0463 ;

$\mathbf{R}_{\mathbf{f}}($ pentane $/ \mathrm{EtOAc}=85 / 15): 0.43$.

\section{1-(Furan-2-yl)-3-methyl-2-((triisopropylsilyl)oxy)but-3-en-1-ol (2m)}

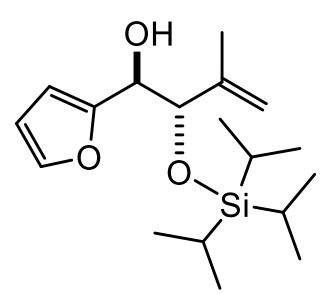

The title compound was synthesized according to General Procedure 1 from furfural (19.2 mg, $0.2 \mathrm{mmol}, 1.0$ equiv.) and triisopropyl((2-methylprop-1-en1 -yl)oxy)silane (137 mg, $0.6 \mathrm{mmol}, 3.0$ equiv.) with a photocatalyst loading of $4 \mathrm{~mol} \%$. Purification by column chromatography on silica gel (pentane/Et $t_{2} \mathrm{O}=98 / 2$ ) gave the two separate diastereomers as colorless oils (combined: $55.4 \mathrm{mg}, 0.172 \mathrm{mmol}, 86 \%$, anti:syn = 72:28). 


\section{Anti:}

${ }^{1} \mathrm{H}$ NMR $\left(400 \mathrm{MHz}, \mathrm{CD}_{2} \mathrm{Cl}_{2}\right): \delta 7.37(\mathrm{~m}, 1 \mathrm{H}), 6.35-6.32(\mathrm{~m}, 1 \mathrm{H}), 6.32-6.28(\mathrm{~m}, 1 \mathrm{H}), 4.98-$ $4.94(\mathrm{~m}, 2 \mathrm{H}), 4.70-4.65(\mathrm{~m}, 1 \mathrm{H}), 4.52(\mathrm{~d}, J=5.3 \mathrm{~Hz}, 1 \mathrm{H}), 2.36(\mathrm{~d}, J=4.0 \mathrm{~Hz}, 1 \mathrm{H}), 1.68(\mathrm{~s}, 3 \mathrm{H})$, $1.02(\mathrm{~s}, 21 \mathrm{H})$;

${ }^{13} \mathrm{C}$ NMR $\left(101 \mathrm{MHz}, \mathrm{CD}_{2} \mathrm{Cl}_{2}\right): \delta 154.6,145.4,142.1,115.1,110.3,108.2,79.5,71.1,18.3,18.3$, 18.2, 13.0;

HRMS $\left(E S I^{+}\right):[\mathrm{M}+\mathrm{Na}]^{+}$347.2013, found 347.2012;

$\mathbf{R}_{\mathbf{f}}($ pentane/EtOAc $=98 / 2): 0.35$.

\section{3-Methyl-1-(thiophen-2-yl)-2-((triisopropylsilyl)oxy)but-3-en-1-ol (2n)}

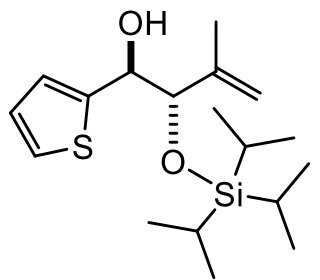

The title compound was synthesized according to General Procedure 1 from thiophene-2-carboxaldehyde $\quad(22.4 \mathrm{mg}, \quad 0.2 \mathrm{mmol}, \quad 1.0$ equiv. $)$ and triisopropyl((2-methylprop-1-en-1-yl)oxy)silane $\quad(137 \mathrm{mg}, \quad 0.6 \mathrm{mmol}$, 3.0 equiv.) with a photocatalyst loading of $4 \mathrm{~mol} \%$. Purification by column chromatography on silica gel (pentane/Et $2 \mathrm{O}=98 / 2$ ) gave the two separate diastereomers as colorless oils (combined: $50.4 \mathrm{mg}, 0.148 \mathrm{mmol}, 74 \%$, anti:syn = 90:10).

\section{Anti:}

${ }^{1} \mathrm{H}$ NMR $\left(400 \mathrm{MHz}, \mathrm{CD}_{2} \mathrm{Cl}_{2}\right): \delta 7.24(\mathrm{dd}, J=5.0,1.3 \mathrm{~Hz}, 1 \mathrm{H}), 6.99-6.97(\mathrm{~m}, 1 \mathrm{H}), 6.96-6.93$ (m, 1H), $4.98-4.95(\mathrm{~m}, 1 \mathrm{H}), 4.92-4.89(\mathrm{~m}, 2 \mathrm{H}), 4.38(\mathrm{~d}, J=5.3 \mathrm{~Hz}, 1 \mathrm{H}), 2.60(\mathrm{~d}, J=3.3 \mathrm{~Hz}$, $1 \mathrm{H}), 1.68(\mathrm{~s}, 3 \mathrm{H}), 1.05-1.02(\mathrm{~m}, 21 \mathrm{H})$;

${ }^{13} \mathrm{C}$ NMR $\left(101 \mathrm{MHz}, \mathrm{CD}_{2} \mathrm{Cl}_{2}\right): \delta$ 145.0, 144.7, 126.7, 125.5, 125.1, 115.3, 81.6, 73.6, 18.8, 18.4, 18.3, 13.1;

HRMS $\left(E S I^{+}\right):[\mathrm{M}+\mathrm{Na}]^{+} 363.1785$, found 363.1785 ;

$\mathbf{R}_{\mathbf{f}}($ pentane $/ \mathrm{EtOAc}=98 / 2): 0.42$ 
tert-Butyl-3-(1-hydroxy-3-methyl-2-((triisopropylsilyl)oxy)but-3-en-1-yl)-indole-1carboxylate (20)

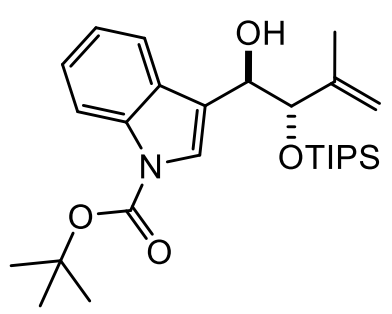

The title compound was synthesized according to General Procedure 1 from tert-butyl 3-formyl-indole-1-carboxylate $(49.1 \mathrm{mg}, \quad 0.2 \mathrm{mmol}$, 1.0 equiv.) and triisopropyl((2-methylprop-1-en-1-yl)oxy)silane (137 mg, $0.6 \mathrm{mmol}, 3.0$ equiv.) with a photocatalyst loading of $4 \mathrm{~mol} \%$. Purification by column chromatography on silica gel (pentane/ $\mathrm{Et}_{2} \mathrm{O}=98 / 2$ ) gave the two separate diastereomers as colorless oils (combined: $87.1 \mathrm{mg}, 0.184 \mathrm{mmol}, 92 \%$, anti:syn = 69:31).

Anti:

${ }^{1} \mathrm{H}$ NMR $\left(400 \mathrm{MHz}, \mathrm{CD}_{2} \mathrm{Cl}_{2}\right): \delta 8.12(\mathrm{~d}, J=8.3 \mathrm{~Hz}, 1 \mathrm{H}), 7.65(\mathrm{~d}, J=7.9 \mathrm{~Hz}, 1 \mathrm{H}), 7.53(\mathrm{~s}, 1 \mathrm{H})$, $7.34-7.28(\mathrm{~m}, 1 \mathrm{H}), 7.24-7.18(\mathrm{~m}, 1 \mathrm{H}), 4.87-4.78(\mathrm{~m}, 2 \mathrm{H}), 4.53(\mathrm{~d}, J=6.5 \mathrm{~Hz}, 1 \mathrm{H}), 3.10-$ $3.04(\mathrm{~m}, 1 \mathrm{H}), 1.72(\mathrm{~s}, 3 \mathrm{H}), 1.68(\mathrm{~s}, 9 \mathrm{H}), 1.09-1.03(\mathrm{~m}, 21 \mathrm{H})$;

${ }^{13} \mathrm{C}$ NMR $\left(101 \mathrm{MHz}, \mathrm{CD}_{2} \mathrm{Cl}_{2}\right): \delta 145.4,136.3,129.4,124.8,124.6,122.9,121.0,120.5,115.8$, $114.6,84.2,80.8,71.5,28.5,18.7,18.4,18.4,13.1$;

HRMS $\left(E S I^{+}\right):[\mathrm{M}+\mathrm{Na}]^{+}$496.2854, found 496.2852;

$\mathbf{R}_{\mathbf{f}}($ pentane/EtOAc $=98 / 2): 0.34$.

(3S,9S,10S,13S,14S)-10,13-Dimethyl-17-oxohexadecahydro-1Hcyclopenta[a]phenanthren-3-yl 3-(1-hydroxy-3-methyl-2-((triisopropylsilyl)oxy)but-3-en1-yl)benzoate (2p)

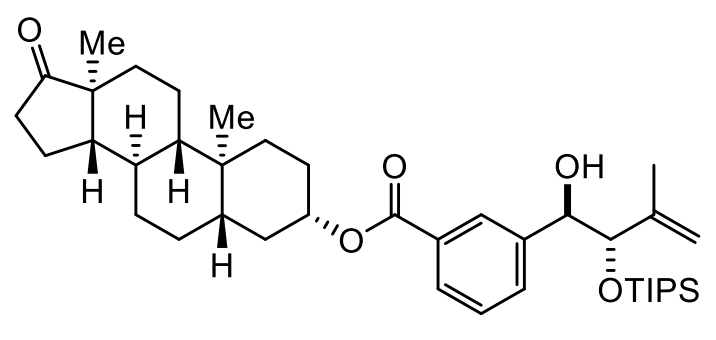

The title compound was synthesized according to General Procedure 1 from $(3 S, 9 S, 10 S, 13 S, 14 S)$ 10,13-dimethyl-17-oxohexadecahydro- $1 \mathrm{H}$ cyclopenta[a]phenanthren-3-yl 3-formylbenzoate (84.5 mg, $0.2 \mathrm{mmol}, 1.0$ equiv.) and triisopropyl((2- 
methylprop-1-en-1-yl)oxy)silane (137 mg, $0.6 \mathrm{mmol}, 3.0$ equiv.) with a photocatalyst loading of $4 \mathrm{~mol} \%$. Purification by column chromatography on silica gel (pentane/EtOAc $=80 / 20$ ) gave the two separate diastereomers as colorless solids (combined: $108.1 \mathrm{mg}, 0.166 \mathrm{mmol}, 83 \%$, anti:syn $=82: 18)$.

Anti:

${ }^{1} \mathrm{H}$ NMR $\left(400 \mathrm{MHz}, \mathrm{CD}_{2} \mathrm{Cl}_{2}\right): 8.00(\mathrm{~d}, \mathrm{~J}=1.8 \mathrm{~Hz}, 1 \mathrm{H}), 7.92-7.88(\mathrm{~m}, 1 \mathrm{H}), 7.56(\mathrm{~d}, \mathrm{~J}=7.6 \mathrm{~Hz}$, $1 \mathrm{H}), 7.38(\mathrm{td}, \mathrm{J}=7.7,1.4 \mathrm{~Hz}, 1 \mathrm{H}), 4.97-4.91(\mathrm{~m}, 1 \mathrm{H}), 4.91(\mathrm{t}, \mathrm{J}=1.7 \mathrm{~Hz}, 1 \mathrm{H}), 4.82(\mathrm{~s}, 1 \mathrm{H})$, $4.73(\mathrm{~d}, \mathrm{~J}=6.1 \mathrm{~Hz}, 1 \mathrm{H}), 4.31(\mathrm{~d}, \mathrm{~J}=6.0 \mathrm{~Hz}, 1 \mathrm{H}), 2.63(\mathrm{~d}, \mathrm{~J}=2.4 \mathrm{~Hz}, 1 \mathrm{H}), 2.39(\mathrm{dd}, \mathrm{J}=18.9$, $8.8 \mathrm{~Hz}, 1 \mathrm{H}), 2.06-1.90(\mathrm{~m}, 4 \mathrm{H}), 1.80(\mathrm{dd}, \mathrm{J}=13.7,10.1 \mathrm{~Hz}, 4 \mathrm{H}), 1.71(\mathrm{~s}, 3 \mathrm{H}), 1.67-1.48(\mathrm{~m}$, $5 \mathrm{H}), 1.45-0.98(\mathrm{~m}, 7 \mathrm{H}), 0.97(\mathrm{~s}, 21 \mathrm{H}), 0.91(\mathrm{~d}, \mathrm{~J}=1.4 \mathrm{~Hz}, 3 \mathrm{H}), 0.85(\mathrm{~d}, \mathrm{~J}=1.4 \mathrm{~Hz}, 3 \mathrm{H}), 0.77$ (td, J = 11.4, $4.0 \mathrm{~Hz}, 1 \mathrm{H})$;

${ }^{13} \mathrm{C}$ NMR $\left(101 \mathrm{MHz}, \mathrm{CD}_{2} \mathrm{Cl}_{2}\right): \delta 221.1,166.4,145.1,141.9,131.9,131.2,129.1,128.8,128.4$, 115.6, 81.8, 76.3, 74.7, 55.0, 52.0, 48.2, 45.3, 37.3, 36.3, 36.3, 35.6, 34.6, 32.2, 31.4, 29.0, $28.1,22.3,21.1,18.5,18.4,18.3,14.2,13.0,12.6$;

HRMS (ESI+): $[\mathrm{M}+\mathrm{Na}]^{+} 673.4259$, found 673.4264 ;

$\mathbf{R}_{\mathbf{f}}($ pentane $/ E t O A c=80 / 20): 0.59$.

\section{1-Cyclohexyl-3-methyl-2-((triisopropylsilyl)oxy)but-3-en-1-ol (3a)}<smiles>C=C(C)[C@H](O[Si](C(C)C)(C(C)C)C(C)C)C(O)C1CCCCC1</smiles>

The title compound was synthesized according to General Procedure 1 from cyclohexanecarboxaldehyde $(22.4 \mathrm{mg}, \quad 0.2 \mathrm{mmol}, \quad 1.0$ equiv. $)$ and triisopropyl((2-methylprop-1-en-1-yl)oxy)silane (137 mg, $0.6 \mathrm{mmol}$, 3.0 equiv.) with a photocatalyst loading of $4 \mathrm{~mol} \%$. Purification by column chromatography on silica gel (pentane/ $\mathrm{Et}_{2} \mathrm{O}=97 / 3$ ) gave the two separate diastereomers as colorless oils (combined: $62.7 \mathrm{mg}, 0.184 \mathrm{mmol}, 92 \%$, anti:syn = 78:22). 
Anti:

${ }^{1} \mathrm{H}$ NMR $\left(400 \mathrm{MHz}, \mathrm{CD}_{2} \mathrm{Cl}_{2}\right): \delta 4.93(\mathrm{~m}, 1 \mathrm{H}), 4.88(\mathrm{~m}, 1 \mathrm{H}), 4.28(\mathrm{~d}, J=4.6 \mathrm{~Hz}, 1 \mathrm{H}), 3.39$ (ddd, $J=8.0,4.6,1.7 \mathrm{~Hz}, 1 \mathrm{H}), 2.40(\mathrm{~d}, J=1.7 \mathrm{~Hz}, 1 \mathrm{H}), 2.01-1.95(\mathrm{~m}, 1 \mathrm{H}), 1.80(\mathrm{~s}, 3 \mathrm{H}), 1.77-1.57$ (m, 4H), $1.35(\mathrm{~m}, 1 \mathrm{H}), 1.28-1.14(\mathrm{~m}, 3 \mathrm{H}), 1.06(\mathrm{dd}, J=5.5,2.7 \mathrm{~Hz}, 21 \mathrm{H}), 0.97-0.85(\mathrm{~m}, 2 \mathrm{H})$;

${ }^{13} \mathrm{C}$ NMR $\left(101 \mathrm{MHz}, \mathrm{CD}_{2} \mathrm{Cl}_{2}\right): \delta$ 145.7, 114.7, 78.8, 78.0, 39.4, 30.2, 29.1, 27.2, 26.5, 26.5, 19.2, 18.4, 18.4, 13.0;

HRMS $\left(\mathrm{ESI}^{+}\right):[\mathrm{M}+\mathrm{Na}]^{+} 363.2690$, found 363.2688 ;

$\mathbf{R}_{\mathbf{f}}($ pentane $/ \mathrm{EtOAc}=97 / 3): 0.23$

\section{2-((Tert-butyldimethylsilyl)oxy)-1-cyclohexyl-2-methylbut-3-en-1-ol (3b)}<smiles>C=CC(C)(O[Si](C)(C)C(C)(C)C)C(O)C1CCCCC1</smiles>

The title compound was synthesized according to General Procedure 1 from cyclohexanecarboxaldehyde ( $24.2 \mu \mathrm{L}, 0.2 \mathrm{mmol}, 1.0$ equiv.) and (but2-en-2-yloxy)(tert-butyl)dimethylsilane (112 mg, $0.6 \mathrm{mmol}, 3.0$ equiv.) with a photocatalyst loading of $4 \mathrm{~mol} \%$. Purification by column chromatography on silica gel (pentane/Et $\mathrm{t}_{2} \mathrm{O}=99 / 1$ ) gave the two separate diastereomers as colorless oils (combined: $33.4 \mathrm{mg}, 0.112 \mathrm{mmol}, 56 \%$, anti:syn = 56:44).

HRMS $\left(\mathrm{ESI}^{+}\right):[\mathrm{M}+\mathrm{Na}]^{+} 321.2220$, found 321.2219 ;

Syn:

${ }^{1} \mathrm{H}$ NMR $\left(400 \mathrm{MHz}, \mathrm{CD}_{2} \mathrm{Cl}_{2}\right): \delta 6.01(\mathrm{dd}, J=17.6,10.9 \mathrm{~Hz}, 1 \mathrm{H}), 5.22-5.11(\mathrm{~m}, 2 \mathrm{H}), 3.05$ (dd, $J=6.2,2.9 \mathrm{~Hz}, 1 \mathrm{H}), 2.30(\mathrm{~d}, J=6.3 \mathrm{~Hz}, 1 \mathrm{H}), 1.87-1.79(\mathrm{~m}, 1 \mathrm{H}), 1.75-1.65(\mathrm{~m}, 1 \mathrm{H}), 1.63-$ $1.58(\mathrm{~m}, 1 \mathrm{H}), 1.54-1.40(\mathrm{~m}, 1 \mathrm{H}), 1.37(\mathrm{~s}, 3 \mathrm{H}), 1.32-1.05(\mathrm{~m}, 7 \mathrm{H}), 0.89(\mathrm{~s}, 9 \mathrm{H}), 0.11(\mathrm{~s}, 3 \mathrm{H})$, $0.09(\mathrm{~s}, 3 \mathrm{H})$;

${ }^{13} \mathrm{C}$ NMR $\left(101 \mathrm{MHz}, \mathrm{CD}_{2} \mathrm{Cl}_{2}\right): \delta 143.1,114.3,82.9,79.8,39.9,33.2,27.7,27.4,27.0,26.8,26.2$, $24.5,18.7,-1.8,-2.0$;

$\mathbf{R}_{\mathbf{f}}\left(\right.$ pentane/ $\left.\mathrm{Et}_{2} \mathrm{O}=99 / 1\right): 0.87$ 
Anti:

${ }^{1} \mathrm{H}$ NMR $\left(400 \mathrm{MHz}, \mathrm{CD}_{2} \mathrm{Cl}_{2}\right): \delta 5.97(\mathrm{dd}, J=17.6,10.9 \mathrm{~Hz}, 1 \mathrm{H}), 5.23-5.10(\mathrm{~m}, 2 \mathrm{H}), 3.08(\mathrm{dd}$, $J=5.3,3.9 \mathrm{~Hz}, 1 \mathrm{H}), 2.43(\mathrm{~d}, J=5.3 \mathrm{~Hz}, 1 \mathrm{H}), 1.90-1.82(\mathrm{~m}, 1 \mathrm{H}), 1.74-1.58(\mathrm{~m}, 2 \mathrm{H}), 1.55-$ $1.43(\mathrm{~m}, 1 \mathrm{H}), 1.33(\mathrm{~s}, 3 \mathrm{H}), 1.23-1.05(\mathrm{~m}, 7 \mathrm{H}), 0.88(\mathrm{~s}, 9 \mathrm{H}), 0.09(\mathrm{~s}, 3 \mathrm{H}), 0.07(\mathrm{~s}, 3 \mathrm{H})$;

${ }^{13} \mathrm{C}$ NMR $\left(101 \mathrm{MHz}, \mathrm{CD}_{2} \mathrm{Cl}_{2}\right): \delta 144.4,114.6,82.5,79.3,39.6,33.0,28.4,27.3,27.0,26.8,26.2$, $21.5,18.7,-1.8,-1.9$;

$\mathbf{R}_{\mathbf{f}}\left(\right.$ pentane/ $\left.\mathrm{Et}_{2} \mathrm{O}=99 / 1\right): 0.79$.

\section{1-Cyclohexyl-2-((triisopropylsilyl)oxy)but-3-en-1-ol (3c)}<smiles>C=CC(O[Si](C(C)C)(C(C)C)C(C)C)C(O)C1CCCCC1</smiles>
silica gel (pentane/Et $2 \mathrm{O}=95 / 5$ ) gave the two separate diastereomers as colorless oils (combined: $48.3 \mathrm{mg}, 0.148 \mathrm{mmol}, 74 \%$, anti:syn = 60:40).

HRMS $\left(E S I^{+}\right):[\mathrm{M}+\mathrm{Na}]^{+}$349.2533, found 349.2537;

Syn:

${ }^{1} \mathrm{H}$ NMR $\left(400 \mathrm{MHz}, \mathrm{CD}_{2} \mathrm{Cl}_{2}\right.$ ): $\delta 5.86(\mathrm{ddd}, J=17.9,10.3,8.0 \mathrm{~Hz}, 1 \mathrm{H}), 5.25-5.14(\mathrm{~m}, 2 \mathrm{H}), 4.23$ (dd, $J=8.0,6.1 \mathrm{~Hz}, 1 \mathrm{H}), 3.11(\mathrm{dt}, J=6.1,4.6 \mathrm{~Hz}, 1 \mathrm{H}), 2.42(\mathrm{~d}, J=4.6 \mathrm{~Hz}, 1 \mathrm{H}), 1.80-1.67(\mathrm{~m}$, $3 \mathrm{H}), 1.67-1.52(\mathrm{~m}, 2 \mathrm{H}), 1.50-1.38(\mathrm{~m}, 1 \mathrm{H}), 1.33-1.10(\mathrm{~m}, 5 \mathrm{H}), 1.09-0.97(\mathrm{~m}, 21 \mathrm{H})$;

${ }^{13} \mathrm{C}$ NMR $\left(101 \mathrm{MHz}, \mathrm{CD}_{2} \mathrm{Cl}_{2}\right): \delta$ 139.9, 117.1, 79.6, 76.5, 39.7, 31.2, 27.4, 27.1, 27.1, 26.8, 18.5, $18.4,13.1$

$\mathbf{R}_{\mathbf{f}}($ pentane/EtOAc $=95 / 5): 0.67$ 
Anti:

${ }^{1} \mathrm{H}$ NMR $\left(400 \mathrm{MHz}, \mathrm{CD}_{2} \mathrm{Cl}_{2}\right): \delta 5.89$ (ddd, $\left.J=17.5,10.4,7.3 \mathrm{~Hz}, 1 \mathrm{H}\right), 5.28-5.19(\mathrm{~m}, 2 \mathrm{H}), 4.33$ (dd, $J=7.3,3.4 \mathrm{~Hz}, 1 \mathrm{H}$ ), 3.36 (ddd, $J=9.0,3.4,1.6 \mathrm{~Hz}, 1 \mathrm{H}$ ), 2.48 (d, $J=1.6 \mathrm{~Hz}, 1 \mathrm{H}$ ), $2.08-$ $1.98(\mathrm{~m}, 1 \mathrm{H}), 1.76-1.55(\mathrm{~m}, 4 \mathrm{H}), 1.41-1.25(\mathrm{~m}, 1 \mathrm{H}), 1.20(\mathrm{dd}, J=12.3,9.1 \mathrm{~Hz}, 3 \mathrm{H}), 1.07(\mathrm{~h}$, $J=3.4,2.9 \mathrm{~Hz}, 21 \mathrm{H}), 1.03-0.89(\mathrm{~m}, 2 \mathrm{H})$;

${ }^{13} \mathrm{C}$ NMR $\left(101 \mathrm{MHz}, \mathrm{CD}_{2} \mathrm{Cl}_{2}\right): \delta 136.8,117.4,79.3,75.6,39.8,30.0,29.1,27.1,26.5,26.4,18.4$, 18.4, 12.9;

$\mathbf{R}_{\mathbf{f}}($ pentane/EtOAc $=95 / 5): 0.51$.

\section{2-((Tert-butyldimethylsilyl)oxy)-1-cyclohexylbut-3-en-1-ol (3d)}<smiles>C=CC(O[Si](C)(C)C(C)(C)C)C(O)C1CCCCC1</smiles>
The title compound was synthesized according to General Procedure 1 from cyclohexanecarboxaldehyde ( $24.2 \mu \mathrm{L}, 0.2 \mathrm{mmol}, 1.0$ equiv.) and tertbutyldimethyl(prop-1-en-1-yloxy)silane (103.4 mg, $0.6 \mathrm{mmol}, 3.0$ equiv.) with a photocatalyst loading of $7 \mathrm{~mol} \%$. Purification by column chromatography on silica gel (pentane $/ \mathrm{Et}_{2} \mathrm{O}=97 / 3$ ) gave the two separate diasteromers as colorless oils (combined: $43.2 \mathrm{mg}, 0.152 \mathrm{mmol}, 76 \%$, anti:syn = 55:45).

HRMS $\left(\mathrm{ESI}^{+}\right):[\mathrm{M}+\mathrm{Na}]^{+}$307.2064, found 307.2061 ;

Syn:

${ }^{1} \mathrm{H}$ NMR $\left(400 \mathrm{MHz}, \mathrm{CD}_{2} \mathrm{Cl}_{2}\right): \delta 5.86$ (ddd, $\left.J=17.4,10.4,7.1 \mathrm{~Hz}, 1 \mathrm{H}\right), 5.25-5.12(\mathrm{~m}, 2 \mathrm{H}), 4.14$ (ddt, $J=5.8,4.9,1.0 \mathrm{~Hz}, 1 \mathrm{H}), 3.06(\mathrm{q}, J=5.5 \mathrm{~Hz}, 1 \mathrm{H}), 2.21(\mathrm{~d}, J=5.8 \mathrm{~Hz}, 1 \mathrm{H}), 1.84-1.78(\mathrm{~m}$, $1 \mathrm{H}), 1.77-1.70(\mathrm{~m}, 2 \mathrm{H}), 1.68-1.57(\mathrm{~m}, 2 \mathrm{H}), 1.46-1.35(\mathrm{~m}, 1 \mathrm{H}), 1.30-1.04(\mathrm{~m}, 5 \mathrm{H}), 0.91(\mathrm{~s}$, $9 \mathrm{H}), 0.09(\mathrm{~s}, 3 \mathrm{H}), 0.06(\mathrm{~s}, 3 \mathrm{H})$;

${ }^{13} \mathrm{C}$ NMR (101 MHz, $\left.\mathrm{CD}_{2} \mathrm{Cl}_{2}\right): \delta 139.9,116.6,79.4,75.4,40.2,30.8,28.0,27.2,27.0,26.8,26.2$, $18.7,-3.6,-4.5$;

$\mathbf{R}_{\mathbf{f}}($ pentane/EtOAc $=95 / 5): 0.64$ 
Anti:

${ }^{1} \mathrm{H}$ NMR $\left(400 \mathrm{MHz}, \mathrm{CD}_{2} \mathrm{Cl}_{2}\right): \delta 5.87$ (ddd, $\left.J=17.3,10.4,6.9 \mathrm{~Hz}, 1 \mathrm{H}\right), 5.28-5.17(\mathrm{~m}, 2 \mathrm{H}), 4.21$ $-4.16(\mathrm{~m}, 1 \mathrm{H}), 3.30-3.23(\mathrm{~m}, 1 \mathrm{H}), 2.27(\mathrm{~d}, J=2.2 \mathrm{~Hz}, 1 \mathrm{H}), 2.01-1.93(\mathrm{~m}, 1 \mathrm{H}), 1.77-1.56$ (m, 4H), $1.44-1.31(\mathrm{~m}, 1 \mathrm{H}), 1.30-1.11(\mathrm{~m}, 3 \mathrm{H}), 1.10-0.93(\mathrm{~m}, 2 \mathrm{H}), 0.90(\mathrm{~s}, 9 \mathrm{H}), 0.08(\mathrm{~s}$, $3 \mathrm{H}), 0.06(\mathrm{~s}, 3 \mathrm{H})$;

${ }^{13} \mathrm{C}$ NMR $\left(101 \mathrm{MHz}, \mathrm{CD}_{2} \mathrm{Cl}_{2}\right): \delta 137.2,117.4,79.1,75.4,39.6,29.5,29.4,27.1,26.6,26.5,26.2$, 18.6, -4.0, -4.7;

$\mathbf{R}_{\mathbf{f}}($ pentane/EtOAc $=95 / 5): 0.56$.

\section{2-((Tert-butyldiphenylsilyl)oxy)-1-cyclohexylbut-3-en-1-ol (3e)}<smiles>C=CC(O[Si](c1ccccc1)(c1ccccc1)C(C)(C)C)C(O)C1CCCCC1</smiles>

The title compound was synthesized according to General Procedure 1 from cyclohexanecarboxaldehyde ( $24.2 \mu \mathrm{L}, 0.2 \mathrm{mmol}, 1.0$ equiv.) and tert-butyldiphenyl(prop-1-en-1-yloxy)silane $\quad(178 \mathrm{mg}, \quad 0.6 \mathrm{mmol}$, 3.0 equiv.) with a photocatalyst loading of $7 \mathrm{~mol} \%$. Purification by column chromatography on silica gel (pentane/ $/ \mathrm{Et}_{2} \mathrm{O}=98 / 2$ ) gave the two separate diastereomers as colorless oils (combined: $67.8 \mathrm{mg}, 0.166 \mathrm{mmol}, 83 \%$, anti:syn $=55: 45)$.

HRMS $\left(\mathrm{ESI}^{+}\right):[\mathrm{M}+\mathrm{Na}]^{+}$431.2370, found 431.2370;

Syn:

${ }^{1} \mathrm{H}$ NMR $\left(400 \mathrm{MHz}, \mathrm{CD}_{2} \mathrm{Cl}_{2}\right)$ : $\delta 7.68(\mathrm{~m}, 4 \mathrm{H}), 7.48-7.32(\mathrm{~m}, 6 \mathrm{H}), 5.84$ (ddd, $J=17.4,10.4,7.9$ $\mathrm{Hz}, 1 \mathrm{H}$ ), 4.90 (dd, $J=10.4,1.7 \mathrm{~Hz}, 1 \mathrm{H}$ ), 4.78 (ddd, $J=17.4,1.7,0.9 \mathrm{~Hz}, 1 \mathrm{H}$ ), 4.16 (dd, $J=8.0$, $5.2 \mathrm{~Hz}, 1 \mathrm{H}), 3.15(\mathrm{q}, J=5.5 \mathrm{~Hz}, 1 \mathrm{H}), 2.25(\mathrm{~d}, J=5.9 \mathrm{~Hz}, 1 \mathrm{H}), 1.89-1.47(\mathrm{~m}, 5 \mathrm{H}), 1.46-1.23$ $(\mathrm{m}, 2 \mathrm{H}), 1.17-1.08(\mathrm{~m}, 3 \mathrm{H}), 1.07(\mathrm{~s}, 9 \mathrm{H}), 0.91(\mathrm{~d}, J=2.2 \mathrm{~Hz}, 1 \mathrm{H})$;

${ }^{13} \mathrm{C}$ NMR $\left(101 \mathrm{MHz}, \mathrm{CD}_{2} \mathrm{Cl}_{2}\right): \delta 138.8,136.7,136.6,134.4,134.1,130.3,130.2,128.2,127.9$, $117.4,79.7,77.1,40.0,30.7,27.8,27.5,27.1,26.9,26.7,19.9$;

$\mathbf{R}_{\mathbf{f}}($ pentane/EtOAc $=97 / 3): 0.51$; 
Anti:

${ }^{1} \mathrm{H}$ NMR $\left(400 \mathrm{MHz}, \mathrm{CD}_{2} \mathrm{Cl}_{2}\right): 7.66(\mathrm{~m}, 4 \mathrm{H}), 7.48-7.33(\mathrm{~m}, 6 \mathrm{H}), 5.89$ (ddd, $J=17.5,10.5,7.3$ $\mathrm{Hz}, 1 \mathrm{H}$ ), 5.11 (ddd, $J=10.5,1.9,1.0 \mathrm{~Hz}, 1 \mathrm{H}$ ), 4.99 (ddd, $J=17.5,1.9,1.0 \mathrm{~Hz}, 1 \mathrm{H}$ ), $4.24-4.19$ (m, 1H), 3.22 (ddd, $J=8.7,3.7,1.8 \mathrm{~Hz}, 1 \mathrm{H}), 2.32(\mathrm{~d}, J=2.1 \mathrm{~Hz}, 1 \mathrm{H}), 1.94-1.84(\mathrm{~m}, 1 \mathrm{H}), 1.72$ $-1.47(\mathrm{~m}, 4 \mathrm{H}), 1.45-1.19(\mathrm{~m}, 3 \mathrm{H}), 1.08(\mathrm{~s}, 9 \mathrm{H}), 0.97-0.76(\mathrm{~m}, 2 \mathrm{H}), 0.69(\mathrm{~m}, 1 \mathrm{H})$;

${ }^{13} \mathrm{C}$ NMR $\left(101 \mathrm{MHz}, \mathrm{CD}_{2} \mathrm{Cl}_{2}\right): \delta 136.6,136.5,136.0,134.3,134.2,130.4,130.2,128.2,128.0$, $118.1,78.8,76.4,39.6,29.6,29.0,27.4,27.0,26.3,26.3,19.7$;

$\mathbf{R}_{\mathbf{f}}($ pentane/EtOAc $=97 / 3): 0.48$.

\section{2-Butoxy-1-cyclohexyl-3-methylbut-3-en-1-ol (3f)}<smiles>C=C(C)C(OCCCC)[C@H](O)C1CCCCC1</smiles>

The title compound was synthesized according to General Procedure 1 from cyclohexanecarboxaldehyde ( $24.2 \mu \mathrm{L}, 0.2 \mathrm{mmol}, 1.0$ equiv.) and 1 (prop-1-en-1-yloxy)butane $(76.9 \mathrm{mg}, \quad 0.6 \mathrm{mmol}, 3.0$ equiv.) with a photocatalyst loading of $4 \mathrm{~mol} \%$. Purification by column chromatography on silica gel (pentane/Et $\mathrm{t}_{2} \mathrm{O}=97 / 3$ ) gave the two separate diastereomers as colorless oils (combined: $43.8 \mathrm{mg}, 0.182 \mathrm{mmol}, 91 \%$, anti:syn $=84: 16)$.

Anti:

${ }^{1} \mathrm{H}$ NMR $\left(400 \mathrm{MHz}, \mathrm{CD}_{2} \mathrm{Cl}_{2}\right): \delta 5.06(\mathrm{t}, J=1.8 \mathrm{~Hz}, 1 \mathrm{H}), 4.97-4.94(\mathrm{~m}, 1 \mathrm{H}), 3.59(\mathrm{~d}, J=7.4 \mathrm{~Hz}$, $1 \mathrm{H}), 3.47-3.38(\mathrm{~m}, 1 \mathrm{H}), 3.36(\mathrm{~d}, J=3.2 \mathrm{~Hz}, 1 \mathrm{H}), 3.19(\mathrm{dt}, J=9.3,6.4 \mathrm{~Hz}, 1 \mathrm{H}), 1.78-1.71(\mathrm{~m}$, $3 \mathrm{H}), 1.70(\mathrm{t}, J=1.2 \mathrm{~Hz}, 3 \mathrm{H}), 1.65-1.47(\mathrm{~m}, 5 \mathrm{H}), 1.41-1.34(\mathrm{~m}, 2 \mathrm{H}), 1.28-1.09(\mathrm{~m}, 5 \mathrm{H}), 0.91$ (t, $J=7.3 \mathrm{~Hz}, 3 \mathrm{H}$ );

${ }^{13} \mathrm{C}$ NMR $\left(101 \mathrm{MHz}, \mathrm{CD}_{2} \mathrm{Cl}_{2}\right): \delta 144.4,116.4,84.9,75.3,68.8,39.5,32.5,30.9,27.3,27.2,26.9$, $26.9,20.1,17.6,14.2$;

HRMS $\left.(\mathrm{ESI})^{+}\right):[\mathrm{M}+\mathrm{Na}]^{+}$263.1982, found 263.1980;

$\mathbf{R}_{\mathbf{f}}\left(\right.$ pentane $\left./ \mathrm{Et}_{2} \mathrm{O}=95 / 5\right): 0.28$. 


\section{1-Cyclohexyl-2-(4-methoxyphenoxy)-3-methylbut-3-en-1-ol (3g)}<smiles>C=C(C)[C@H](Oc1ccc(OC)cc1)C(O)C1CCCCC1</smiles>

The title compound was synthesized according to General Procedure 1 from cyclohexanecarboxaldehyde (24.2 $\mu \mathrm{L}, 0.2 \mathrm{mmol}, 1.0$ equiv.) and 1-methoxy-4-((2-methylprop-1-en-1-yl)oxy)benzene (107 mg, 0.6 mmol,

3.0 equiv.) with a photocatalyst loading of $4 \mathrm{~mol} \%$. Purification by column chromatography on silica gel (pentane/Et $\mathrm{t}_{2} \mathrm{O}=97 / 3$ ) gave the two separate diastereomers as colorless oils (combined: $43.6 \mathrm{mg}, 0.150 \mathrm{mmol}, 75 \%$, anti:syn = 83:17).

Anti:

${ }^{1} \mathrm{H}$ NMR $\left(400 \mathrm{MHz}, \mathrm{CD}_{2} \mathrm{Cl}_{2}\right): \delta 6.87-6.82(\mathrm{~m}, 2 \mathrm{H}), 6.81-6.76(\mathrm{~m}, 2 \mathrm{H}), 5.12(\mathrm{p}, \mathrm{J}=1.6 \mathrm{~Hz}$, $1 \mathrm{H}), 5.07$ (dp, $J=1.7,0.8 \mathrm{~Hz}, 1 \mathrm{H}), 4.45$ (d, $J=7.3 \mathrm{~Hz}, 1 \mathrm{H}), 3.74$ (s, 3H), 3.59 (dt, J = 7.2, 3.6 $\mathrm{Hz}, 1 \mathrm{H}), 1.80-1.77(\mathrm{~m}, 2 \mathrm{H}), 1.74(\mathrm{dd}, J=1.6,0.9 \mathrm{~Hz}, 3 \mathrm{H}), 1.69-1.59(\mathrm{~m}, 2 \mathrm{H}), 1.43-0.84(\mathrm{~m}$, $7 \mathrm{H})$;

${ }^{13} \mathrm{C}$ NMR $\left(101 \mathrm{MHz}, \mathrm{CD}_{2} \mathrm{Cl}_{2}\right): \delta 154.7,152.5,143.8,117.5,116.7,115.0,83.5,75.3,56.1,39.5$, $31.0,27.2,27.2,26.8,26.8,18.1$;

HRMS $\left(\mathrm{ESI}^{+}\right):[\mathrm{M}+\mathrm{Na}]^{+}$313.1774, found 313.1770;

$\mathbf{R}_{\mathbf{f}}($ pentane/EtOAc $=97 / 3): 0.37$.

\section{2-(Benzyloxy)-1-cyclohexyl-3-methylbut-3-en-1-ol (3h)}<smiles>C=C(C)[C@H](OCc1ccccc1)C(O)C1CCCCC1</smiles>

The title compound was synthesized according to General Procedure 1 from cyclohexanecarboxaldehyde $(24.2 \mu \mathrm{L}, \quad 0.2 \mathrm{mmol}, \quad 1.0$ equiv. $)$ and (( 2 methylprop-1-en-1-yl)oxy)methyl)benzene (97.3 mg, 0.6 mmol, 3.0 equiv.) with a photocatalyst loading of $4 \mathrm{~mol} \%$. Purification by column chromatography on silica gel (pentane/Et $2 \mathrm{O}=95 / 5$ ) gave the two separate diastereomers as colorless oils (combined: $43.9 \mathrm{mg}, 0.160 \mathrm{mmol}, 80 \%$, anti:syn = 82:18). 
Anti:

${ }^{1} \mathrm{H}$ NMR $\left(400 \mathrm{MHz}, \mathrm{CD}_{2} \mathrm{Cl}_{2}\right): \delta 7.38-7.25(\mathrm{~m}, 5 \mathrm{H}), 5.17-5.16(\mathrm{~m}, 1 \mathrm{H}), 5.03-5.02(\mathrm{~m}, 1 \mathrm{H})$, $4.51(\mathrm{~d}, J=11.7 \mathrm{~Hz}, 1 \mathrm{H}), 4.26(\mathrm{~d}, J=11.7 \mathrm{~Hz}, 1 \mathrm{H}), 3.73(\mathrm{~d}, J=7.6 \mathrm{~Hz}, 1 \mathrm{H}), 3.45$ (dt, $J=6.9$, $2.9 \mathrm{~Hz}, 1 \mathrm{H}), 1.78(\mathrm{t}, J=1.2 \mathrm{~Hz}, 3 \mathrm{H}), 1.76-1.55(\mathrm{~m}, 7 \mathrm{H}), 1.32-1.04(\mathrm{~m}, 4 \mathrm{H})$;

${ }^{13} \mathrm{C}$ NMR $\left(101 \mathrm{MHz}, \mathrm{CD}_{2} \mathrm{Cl}_{2}\right): \delta 143.7,139.1,128.8,128.5,128.1,117.3,84.0,75.2,70.7,39.4$, 30.9, 27.2, 27.2, 26.8, 26.6, 17.6;

HRMS $\left(\mathrm{ESI}{ }^{+}\right):[\mathrm{M}+\mathrm{Na}]^{+}$297.1825, found 297.1822;

$\mathbf{R}_{\mathbf{f}}($ pentane/EtOAc $=95 / 5): 0.27$

1-Cyclohexyl-2-(dodecylthio)-3-methylbut-3-en-1-ol (3i)<smiles>C=C(C)C(SCCC)C(O)C1CCCCC1</smiles>

The title compound was synthesized according to General Procedure 1 from cyclohexanecarboxaldehyde $(24.2 \mu \mathrm{L}, 0.2 \mathrm{mmol}, 1.0$ equiv.) and dodecyl(2-methylprop-1-en-1-yl)sulfane (153.9 mg, $0.6 \mathrm{mmol}, 3.0$ equiv.) with a photocatalyst loading of $4 \mathrm{~mol} \%$. Purification by column chromatography on silica gel (pentane/EtOAc $=98 / 2$ ) gave the two separate diastereomers as yellow oils (combined: $28.0 \mathrm{mg}, 0.076 \mathrm{mmol}, 38 \%$, anti:syn = 59:41).

HRMS $\left(E S I^{+}\right):[\mathrm{M}+\mathrm{Na}]^{+}$391.3005, found 391.3013;

Syn:

${ }^{1} \mathrm{H}$ NMR $\left(400 \mathrm{MHz}, \mathrm{CDCl}_{3}\right) \delta 4.98-4.79(\mathrm{~m}, 2 \mathrm{H}), 3.49-3.08(\mathrm{~m}, 2 \mathrm{H}), 2.44-2.33(\mathrm{~m}, 2 \mathrm{H}), 1.83$ $-1.79(\mathrm{~m}, 3 \mathrm{H}), 1.73-1.26(\mathrm{~m}, 32 \mathrm{H}), 0.90-0.86(\mathrm{~m}, 3 \mathrm{H})$;

${ }^{13} \mathrm{C}$ NMR (101 MHz, $\left.\mathrm{CDCl}_{3}\right) \delta 115.9,75.1,55.8,40.2,39.8,32.5,31.5,31.1,30.2,30.2,30.2$, $30.1,29.9,29.9,29.8,29.5,27.1,27.1,26.7,26.2,23.3,18.6,14.5$;

$\mathbf{R}_{\mathbf{f}}($ pentane/EtOAc = 98/2): 0.42. 


\section{Anti:}

${ }^{1} \mathrm{H}$ NMR $\left(400 \mathrm{MHz}, \mathrm{CDCl}_{3}\right) \delta 4.96(\mathrm{~d}, J=11.1 \mathrm{~Hz}, 2 \mathrm{H}), 3.52-3.43(\mathrm{~m}, 1 \mathrm{H}), 3.15(\mathrm{q}, J=13.3$ $\mathrm{Hz}, 2 \mathrm{H}), 2.53-2.46(\mathrm{~m}, 1 \mathrm{H}), 2.45-2.38(\mathrm{~m}, 2 \mathrm{H}), 2.13-2.03(\mathrm{~m}, 1 \mathrm{H}), 1.85-1.66(\mathrm{~m}, 6 \mathrm{H})$, $1.56-1.51(\mathrm{~m}, 2 \mathrm{H}), 1.32-1.09(\mathrm{~m}, 24 \mathrm{H}), 0.88(\mathrm{t}, J=6.8 \mathrm{~Hz}, 3 \mathrm{H})$;

${ }^{13} \mathrm{C}$ NMR $\left(101 \mathrm{MHz}, \mathrm{CDCl}_{3}\right) \delta 143.8,115.5,73.9,44.3,40.0,38.1,32.5,31.6,30.2,30.2,30.2$, $30.1,29.9,29.8,29.8,29.8,29.5,28.6,27.2,27.0,26.8,23.3,14.5$;

$\mathbf{R}_{\mathbf{f}}($ pentane/EtOAc $=98 / 2): 0.23$.

\section{1-Cyclohexyl-2-((trimethylsilyl)oxy)but-3-en-1-ol (3j)}

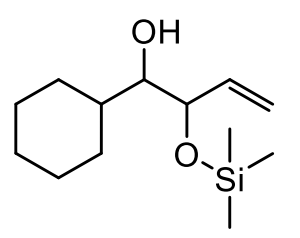

The title compound was synthesized according to General Procedure 2 from cyclohexanecarboxaldehyde $(24.2 \mu \mathrm{L}, \quad 0.2 \mathrm{mmol}, \quad 1.0$ equiv. $)$ and allyloxytrimethylsilane $(78.0 \mathrm{mg}, 0.6 \mathrm{mmol}, 3.0$ equiv.) Purification by column chromatography on silica gel (pentane/Et $2 \mathrm{O}=97 / 3$ ) gave the two separate diastereomers as colorless oils (combined: $29.6 \mathrm{mg}, 0.122 \mathrm{mmol}, 61 \%$, anti:syn $=54: 46$ ).

HRMS $\left(\mathrm{ESI}^{+}\right):[\mathrm{M}+\mathrm{Na}]^{+} 265.1594$, found 265.1592;

Syn:

${ }^{1} \mathrm{H}$ NMR $\left(400 \mathrm{MHz}, \mathrm{CD}_{2} \mathrm{Cl}_{2}\right): \delta 5.84(\mathrm{ddd}, J=17.1,10.4,6.6 \mathrm{~Hz}, 1 \mathrm{H}), 5.26-5.13(\mathrm{~m}, 2 \mathrm{H}), 4.15$ (ddt, $J=6.3,5.0,1.2 \mathrm{~Hz}, 1 \mathrm{H}), 3.06(\mathrm{q}, J=5.3 \mathrm{~Hz}, 1 \mathrm{H}), 2.22(\mathrm{~d}, J=5.5 \mathrm{~Hz}, 1 \mathrm{H}), 1.82-1.71(\mathrm{~m}$, $3 \mathrm{H}), 1.65-1.57(\mathrm{~m}, 2 \mathrm{H}), 1.39(\mathrm{ddt}, J=11.2,5.7,2.8 \mathrm{~Hz}, 1 \mathrm{H}), 1.22-1.09(\mathrm{~m}, 5 \mathrm{H}), 0.12(\mathrm{~s}, 9 \mathrm{H})$;

${ }^{13} \mathrm{C}$ NMR $\left(101 \mathrm{MHz}, \mathrm{CD}_{2} \mathrm{Cl}_{2}\right): \delta$ 139.7, 116.5, 78.9, 75.1, 40.1, 30.8, 27.9, 27.1, 27.0, 26.8, 0.6;

$\mathbf{R}_{\mathbf{f}}($ pentane/Et $2 \mathrm{O}=97 / 3): 0.42$.

\section{Anti:}

${ }^{1} \mathrm{H}$ NMR $\left(400 \mathrm{MHz}, \mathrm{CD}_{2} \mathrm{Cl}_{2}\right): \delta 5.88(\mathrm{ddd}, J=17.3,10.4,6.9 \mathrm{~Hz}, 1 \mathrm{H}), 5.28-5.17(\mathrm{~m}, 2 \mathrm{H}), 4.16$ (ddt, $J=6.8,4.3,1.1 \mathrm{~Hz}, 1 \mathrm{H}$ ), 3.24 (ddd, $J=7.8,4.3,2.4 \mathrm{~Hz}, 1 \mathrm{H}$ ), 2.25 (d, $J=2.5 \mathrm{~Hz}, 1 \mathrm{H}$ ), 2.00 
$-1.91(\mathrm{~m}, 1 \mathrm{H}), 1.74-1.61(\mathrm{~m}, 3 \mathrm{H}), 1.42-1.30(\mathrm{~m}, 1 \mathrm{H}), 1.31-1.16(\mathrm{~m}, 4 \mathrm{H}), 1.11-0.89(\mathrm{~m}$, $2 \mathrm{H}), 0.11(\mathrm{~s}, 9 \mathrm{H})$;

${ }^{13} \mathrm{C}$ NMR $\left(101 \mathrm{MHz}, \mathrm{CD}_{2} \mathrm{Cl}_{2}\right): \delta$ 137.3, 117.3, 79.1, 75.2, 39.6, 29.5, 29.2, 27.1, 26.6, 26.5, 0.4;

$\mathbf{R}_{\mathbf{f}}\left(\right.$ pentane $\left./ \mathrm{Et}_{2} \mathrm{O}=97 / 3\right): 0.34$.

\section{2-Butoxy-1-cyclohexylbut-3-en-1-ol (3k)}

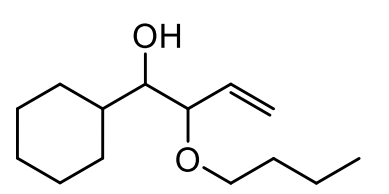

The title compound was synthesized according to General Procedure 2 from cyclohexanecarboxaldehyde ( $24.2 \mu \mathrm{L}, 0.2 \mathrm{mmol}, 1.0$ equiv.) and allyl butyl ether $(87.5 \mu \mathrm{L}, 0.6 \mathrm{mmol}, 3.0$ equiv.) Purification by column chromatography on silica gel (pentane/ $\mathrm{Et}_{2} \mathrm{O}=97 / 3$ ) gave the two separate diastereomers as colorless oils (combined: $38.0 \mathrm{mg}, 0.168 \mathrm{mmol}, 84 \%$, anti:syn $=54: 46$ ).

HRMS $\left(\mathrm{ESI}^{+}\right):[\mathrm{M}+\mathrm{Na}]^{+}$249.1825, found 249.1823;

Syn:

${ }^{1} \mathrm{H}$ NMR $\left(400 \mathrm{MHz}, \mathrm{CD}_{2} \mathrm{Cl}_{2}\right): \delta 5.73-5.63(\mathrm{~m}, 1 \mathrm{H}), 5.28(\mathrm{~d}, J=0.8 \mathrm{~Hz}, 1 \mathrm{H}), 5.26(\mathrm{dd}, J=1.9$, $0.7 \mathrm{~Hz}, 1 \mathrm{H}$ ), 3.63 (ddt, $J=7.8,6.9,0.8 \mathrm{~Hz}, 1 \mathrm{H}), 3.53(\mathrm{dt}, J=9.3,6.5 \mathrm{~Hz}, 1 \mathrm{H}), 3.27-3.23(\mathrm{~m}$, $1 \mathrm{H}), 3.22-3.17(\mathrm{~m}, 1 \mathrm{H}), 2.47(\mathrm{~d}, J=3.4 \mathrm{~Hz}, 1 \mathrm{H}), 1.79-1.03(\mathrm{~m}, 15 \mathrm{H}), 0.91(\mathrm{t}, J=7.4 \mathrm{~Hz}, 3 \mathrm{H})$;

${ }^{13} \mathrm{C}$ NMR $\left(101 \mathrm{MHz}, \mathrm{CD}_{2} \mathrm{Cl}_{2}\right): \delta$ 136.7, 118.9, 82.9, 78.2, 68.9, 39.9, 32.5, 31.0, 27.2, 27.1, 27.0, 26.8, 20.0, 14.2;

$\mathbf{R}_{\mathbf{f}}($ pentane/EtOAc = 95/5): 0.72;

Anti:

${ }^{1} \mathrm{H}$ NMR $\left(400 \mathrm{MHz}, \mathrm{CD}_{2} \mathrm{Cl}_{2}\right): \delta 5.71(\mathrm{ddd}, J=17.3,10.5,8.1 \mathrm{~Hz}, 1 \mathrm{H}), 5.25-5.21(\mathrm{~m}, 1 \mathrm{H}), 5.18$ (ddd, $J=17.4,2.0,0.9 \mathrm{~Hz}, 1 \mathrm{H}$ ), 3.61 (ddt, $J=8.0,4.4,0.8 \mathrm{~Hz}, 1 \mathrm{H}$ ), $3.45-3.36(\mathrm{~m}, 1 \mathrm{H}$ ), 3.31 (ddd, $J=7.0,3.7,1.5 \mathrm{~Hz}, 1 \mathrm{H}$ ), 3.21 (dt, $J=9.3,6.5 \mathrm{~Hz}, 1 \mathrm{H}$ ), $2.04(\mathrm{~d}, J=2.8 \mathrm{~Hz}, 1 \mathrm{H}$ ), $1.91-$ $0.89(\mathrm{~m}, 15 \mathrm{H}), 0.83(\mathrm{t}, J=7.3 \mathrm{~Hz}, 3 \mathrm{H})$; 
${ }^{13} \mathrm{C}$ NMR $\left(101 \mathrm{MHz}, \mathrm{CD}_{2} \mathrm{Cl}_{2}\right): \delta$ 135.5, 119.5, 82.4, 77.3, 68.7, 39.7, 32.5, 29.5, 29.0, 27.1, 26.7, 26.5, 20.0, 14.2;

$\mathbf{R}_{\mathbf{f}}($ pentane/EtOAc $=95 / 5): 0.59$ 


\section{Mechanistic Analysis}

\section{Linear Scan Voltammetry}

Linear scan voltammetry measurements were performed on a $\mathrm{CH}$ Instruments Electrochemical Analyzer (CHI600E) using a glassy carbon working electrode, a Pt wire counter electrode, and a ferrocene/ferrocenium reference electrode. Voltammograms were taken at room temperature in a $100 \mathrm{mM}$ MeCN solution of tetrabutylammoniumhexafluorophosphate $\left(\mathrm{Bu}_{4} \mathrm{NPF}_{6}\right)$ containing $1 \mathrm{mM}$ of the designated substance (Figures S3/S4, 3mM for (((2-methylprop-1-en-1yl)oxy)methyl)benzene, Figure S5).

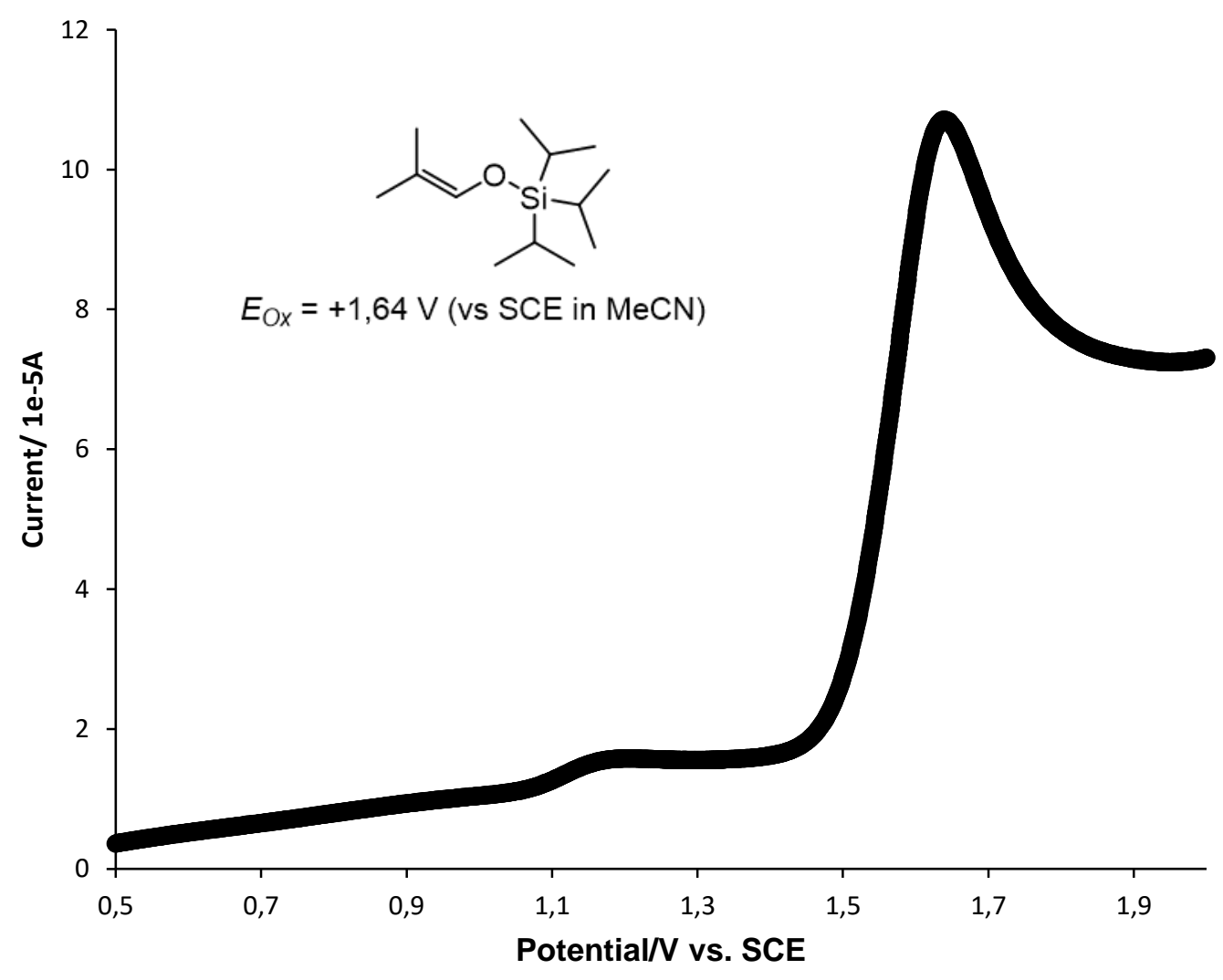

Figure S3: Linear scan voltammogram of triisopropyl((2-methylprop-1-en-1-yl)oxy)silane. 


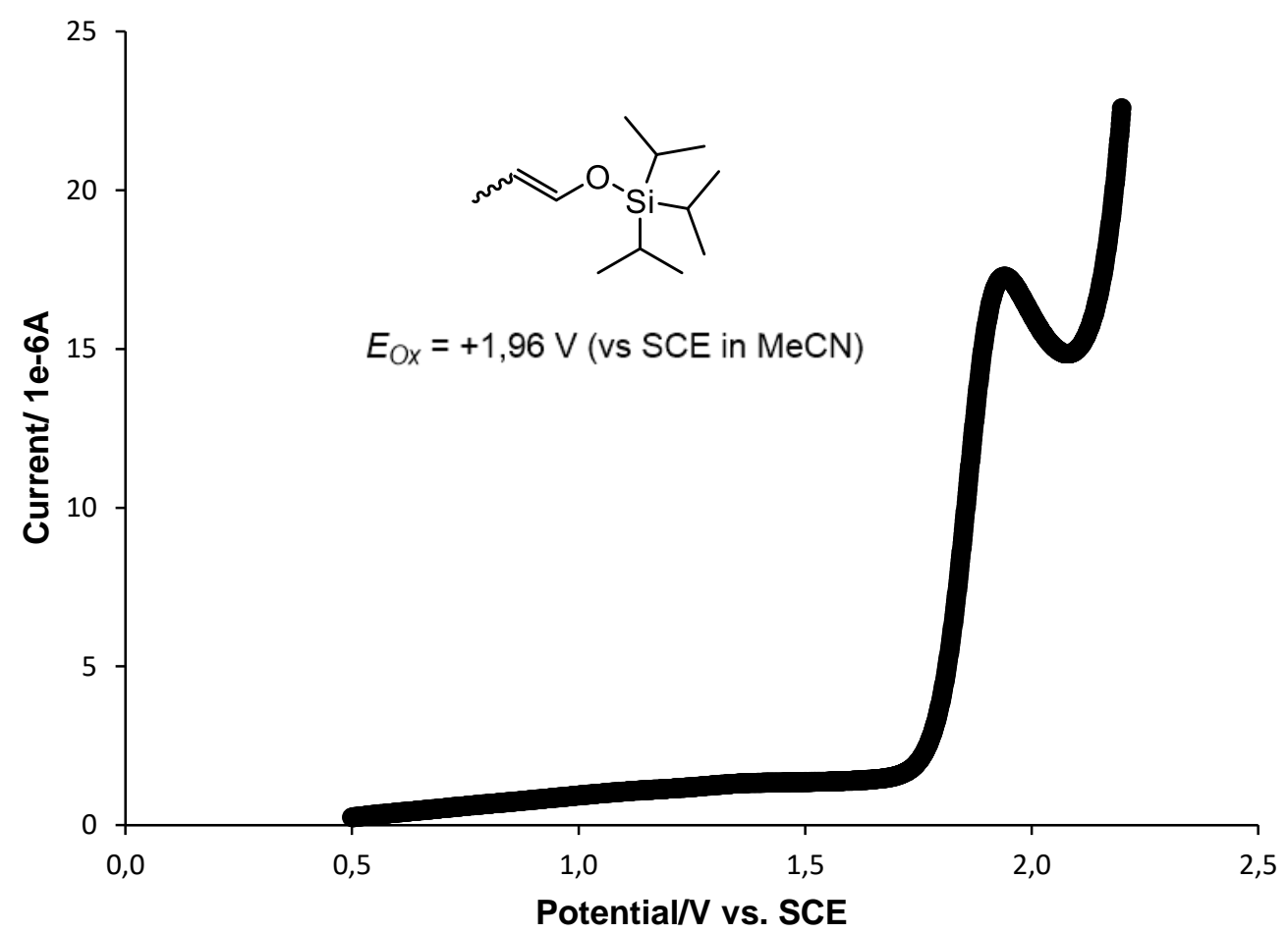

Figure S4: Linear scan voltammogram of triisopropyl(prop-1-en-1-yloxy)silane.

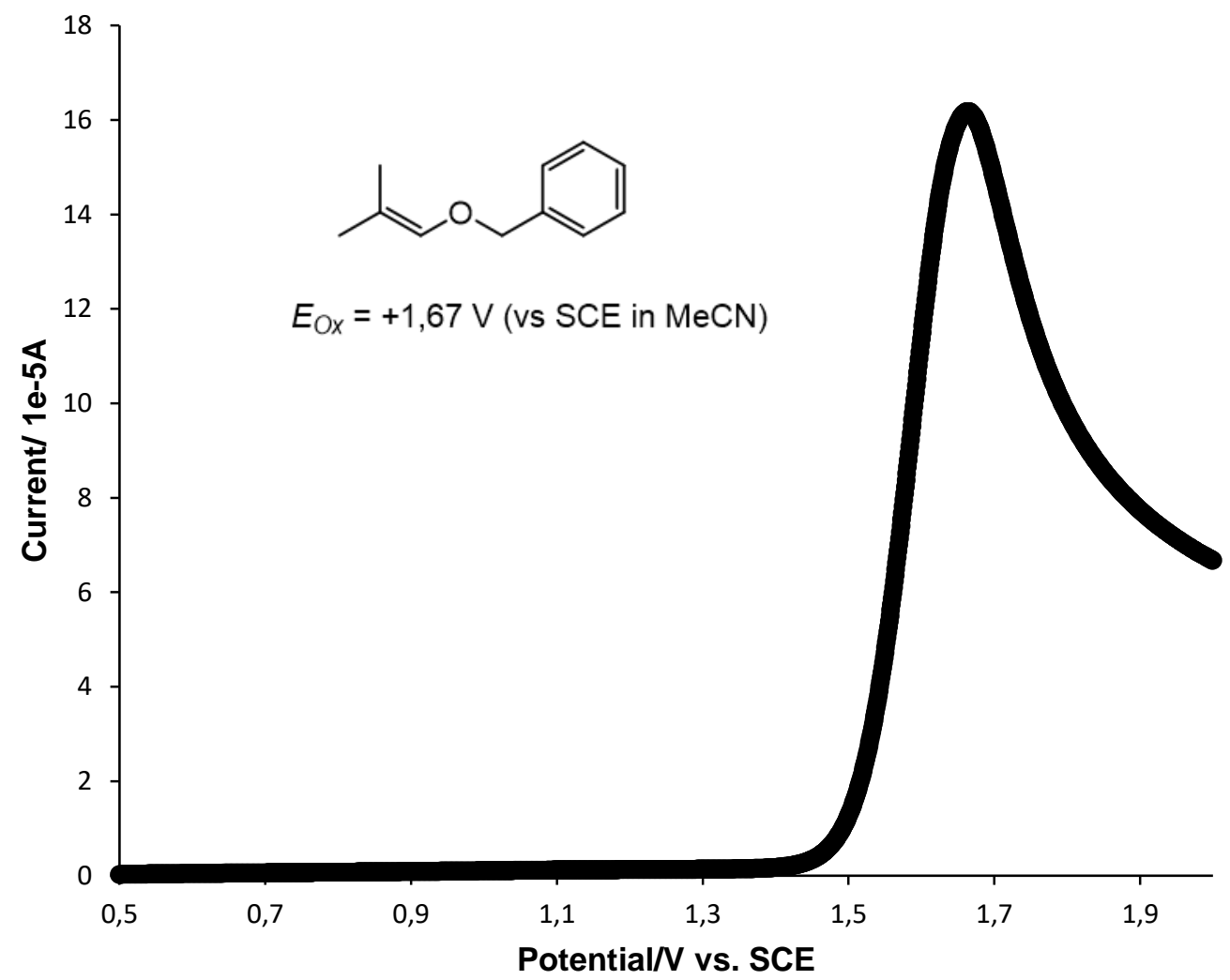

Figure S5: Linear scan voltammogram of (((2-methylprop-1-en-1-yl)oxy)methyl)benzene. 


\section{Quantum Yield}

\section{Determination of the light intensity at $425 \mathrm{~nm}$ :}

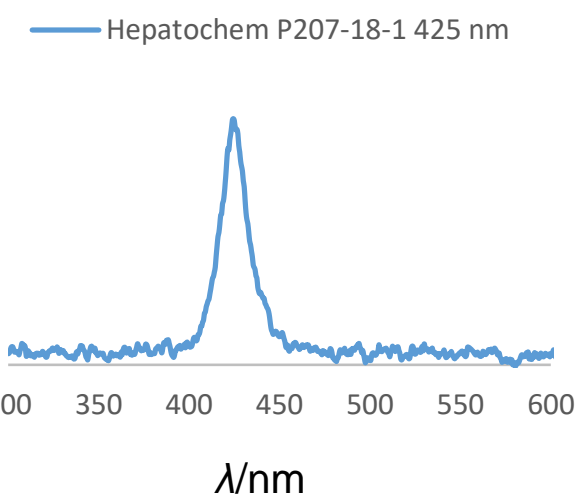

Figure S6: Emission spectrum of the $425 \mathrm{~nm}$ LED used for the quantum yield determination.

Following a literature procedure of Yoon, ${ }^{11}$ the photon flux of the LED $\left(18 \mathrm{~W}, \lambda_{\max }=425 \mathrm{~nm}\right)$ was determined by standard ferrioxalate actinometry. ${ }^{12,13 a, b}$ A solution of ferrioxalate $(0.15 \mathrm{M})$ was prepared by dissolving potassium ferrioxalate hydrate $(737 \mathrm{mg})$ in aq. $\mathrm{H}_{2} \mathrm{SO}_{4}(0.05 \mathrm{M}$, $10 \mathrm{~mL})$. A buffered solution of 1,10-phenanthroline was prepared by dissolving 1,10phenanthroline hydrate $(25.0 \mathrm{mg})$ and sodium acetate $(5.63 \mathrm{~g})$ in aq. $\mathrm{H}_{2} \mathrm{SO}_{4}(0.5 \mathrm{M}, 25 \mathrm{~mL})$. Both solutions were stored in the dark. To determine the photon flux of the LED, the ferrioxalate solution ( $1.0 \mathrm{~mL}$ ) was placed in a $4.0 \mathrm{~mL}$ vial and irradiated for $30 \mathrm{~s}$ at $\lambda_{\max }=425 \mathrm{~nm}$ (see Figure S6 for the emission spectrum of the LED used). After irradiation, the phenanthroline solution $(175 \mu \mathrm{L})$ was added to the vial and the mixture was allowed to stir in the dark for $1 \mathrm{~h}$ to allow the ferrous ions to completely coordinate to the phenanthroline. The solution was transferred to a quartz cuvette and the absorption of the solution was measured at $510 \mathrm{~nm}$. A non-irradiated sample was also prepared and the absorption at $510 \mathrm{~nm}$ was measured. Each sample preparation and measurement was repeated two more times. The average of the absorption of the irradiated and non-irradiated samples was determined and used to calculate the conversion applying eq. 1.

$$
n\left(\mathrm{Fe}^{2+}\right)=\frac{V \cdot \Delta A(510 \mathrm{~nm})}{l \cdot \varepsilon}
$$

where $\mathrm{V}$ is the total volume $(0.001175 \mathrm{~L})$ of the solution after addition of phenanthroline, $\Delta A$ is the difference in absorption at $510 \mathrm{~nm}$ between the irradiated and non-irradiated solutions, / is the path length $(1.0 \mathrm{~cm})$, and $\varepsilon$ is the molar absorptivity of the ferrioxalate actinometer at 510 $\mathrm{nm}\left(11100 \mathrm{~L} \mathrm{~mol}^{-1} \mathrm{~cm}^{-1}\right) .{ }^{12}$ The photon flux $\left(\Phi_{\mathrm{q}}\right)$ can be calculated using eq. 2 ,

$$
\Phi_{\mathrm{q}}=\frac{n\left(\mathrm{Fe}^{2+}\right)}{\Phi_{\mathrm{F}} \cdot t \cdot f}
$$


where $\Phi_{\mathrm{F}}$ is the quantum yield for the ferrioxalate actinometer $\left(\sim 1.12 \text { at } \lambda_{\mathrm{ex}}=425 \mathrm{~nm}\right)^{14}, t$ is the irradiation time (30 s), and $f$ is the fraction of light absorbed at $\lambda_{\mathrm{ex}}=425 \mathrm{~nm}$ by the ferrioxalate actinometer. This value is calculated using eq. 3 where $A(425 \mathrm{~nm})$ is the absorption of the ferrioxalate solution at $425 \mathrm{~nm}$. An absorption spectrum gave an $A(425 \mathrm{~nm})$ value of $>3$, indicating that the fraction of absorbed light $(f)$ is $>0.999$.

$$
f=1-10^{-A(425 \mathrm{~nm})}
$$

The photon flux was calculated (average of three experiments) to be $6.05 \cdot 10^{-9}$ einsteins $\mathrm{s}^{-1}$.

\section{Determination of the reaction's quantum yield:}
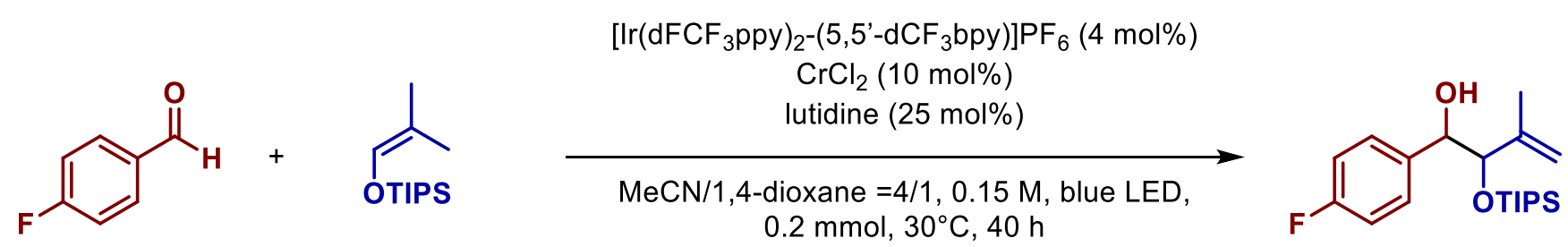

To an oven-dried $4.0 \mathrm{~mL}$ reaction vial was added $\left[\operatorname{Ir}\left(\mathrm{dFCF}_{3} \mathrm{ppy}\right)_{2}-\left(5,5^{\prime}-\mathrm{dCF}_{3} \mathrm{bpy}\right) \mathrm{PF}_{6}(6.9 \mathrm{mg}\right.$, $6.0 \mu \mathrm{mol}, 4 \mathrm{~mol} \%$ ). The vial was transferred to an argon-filled glovebox and $\mathrm{CrCl}_{2}(1.7 \mathrm{mg}$, $0.01 \mathrm{mmol}, 10 \mathrm{~mol} \%$ ) was added. After sealing and removing the vial from the glovebox, under argon were added degassed MeCN $(0.80 \mathrm{~mL})$, 1,4-dioxane $(0.21 \mathrm{~mL})$, lutidine $(4.4 \mu \mathrm{L}$, $0.04 \mathrm{mmol}, 25 \mathrm{~mol} \%)$, triisopropyl((2-methylprop-1-en-1-yl)oxy)silane (102.8 mg, $0.45 \mathrm{mmol}$, 3.0 equiv.) and 4-fluoromethylbenzaldehyde (18.6 mg, $0.15 \mathrm{mmol}, 1$ equiv.). The reaction mixture was irradiated with a single blue LED (18 W, $\left.\lambda_{\max }=425 \mathrm{~nm}\right)$ for $2 \mathrm{~h}$. After irradiation, the reaction mixture was quenched with Rochelle's salt and the phases were separated. The aqueous phase was extracted with EtOAc and the combined organic phases were dried over $\mathrm{MgSO}_{4}$ and concentrated under reduced pressure. The yield was determined $\left({ }^{19} \mathrm{~F}\right.$ NMR analysis using 4-fluorotoluene as an internal standard) to be $11 \%(\mathrm{dr}=78: 22)\left(1.65 \cdot 10^{-5} \mathrm{~mol}\right)$. The reaction quantum yield $(\Phi)$ was determined using eq. 4 where the photon flux is $6.05 \cdot 10^{-9}$ einsteins $\mathrm{s}^{-1}$ (determined by actinometry as described above), $t$ is the reaction time $(2 \mathrm{~h})$ and $f_{\mathrm{R}}$ is the fraction of incident light absorbed by the reaction mixture, determined using eq 4. An absorption spectrum of the reaction mixture gave an absorbance value of $>3$ at $425 \mathrm{~nm}$, indicating that essentially all the incident light $\left(f_{R}>0.999\right)$ is absorbed by the photocatalyst.

$$
\Phi=\frac{n \text { (product) }}{\Phi_{\mathrm{q}} \cdot t \cdot f_{\mathrm{R}}}
$$

The reaction quantum yield $(\Phi)$ was thus determined to be $\Phi=0.38$. 


\section{Stern-Volmer}

Stern-Volmer luminescence quenching analysis was conducted using a Jasco FP-8300 spectrofluorometer. The following parameters were employed: Excitation bandwidth $=5 \mathrm{~nm}$, data interval $=0.5 \mathrm{~nm}$, scan speed $=1000 \mathrm{~nm} \mathrm{~min}^{-1}$, response time $=0.2 \mathrm{~s}$.

All samples used in the luminescence quenching-based screening studies were prepared under oxygen-free conditions. The photocatalyst and potential quenchers were weighed into vials and placed inside a glovebox under a positive pressure of argon. MeCN was degassed by argon sparging for $1 \mathrm{~h}$ and also placed inside along with micropipettes and their tips, cuvettes, empty vials and waste containers. Stern-Volmer luminescence quenching studies were carried out

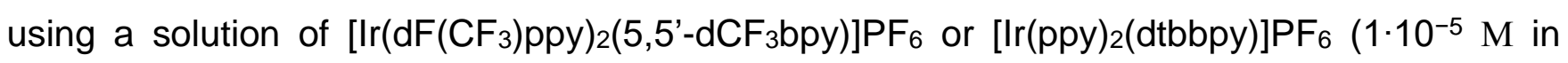
$\mathrm{MeCN}$ ), respectively and variable concentrations of the potential quenchers at rt under an argon atmosphere. The samples were prepared in $1.4 \mathrm{~mL}$ quartz cuvettes and equipped with PTFE stoppers inside an argon-filled glovebox. The solutions were irradiated at $420 \mathrm{~nm}$ and the luminescence was measured at $560 \mathrm{~nm}$. The ratio of $10 / l$ was plotted as a function of the quencher concentration $c$ (Quencher) $\left(l_{0}=\right.$ emission intensity of the photocatalyst in isolation at the specified wavelength; $I=$ observed emission intensity of the photocatalyst with added quencher). The solution of potassium triisopropylsilanethiolate was prepared in situ by stirring triisopropylsilanethiol (1.0 equiv.) and $\mathrm{K}_{2} \mathrm{HPO}_{4}$ (1.0 equiv.) in MeCN overnight, followed by filtration under an atmosphere of argon. The conversion was not determined, therefore the absolute value of the Stern-Volmer constant is not meaningful.

The results clearly show that all three different silyl enols ethers tested clearly quench the excited state of the photocatalyst $\left[\operatorname{Ir}\left(\mathrm{dF}_{(}\left(\mathrm{CF}_{3}\right) \mathrm{ppy}\right)_{2}\left(5,5^{\prime}-\mathrm{dCF}_{3} \mathrm{bpy}\right)\right] \mathrm{PF} 6$, while the aldehyde does not show quenching. The electron-richer substituted enol ether (Figure S7) quenches far better than the two non-substituted ones (Figure S8/S9), which is in accordance with our proposal and the measured oxidation potentials. In addition it was shown, that the HAT catalyst, potassium triisopropylsilanethiol, quenches the excited state of $\left[\operatorname{Ir}(\mathrm{ppy})_{2}(\mathrm{dtbbpy})\right]_{\mathrm{PF}} \mathrm{F}_{6}$ (Figure S10). 


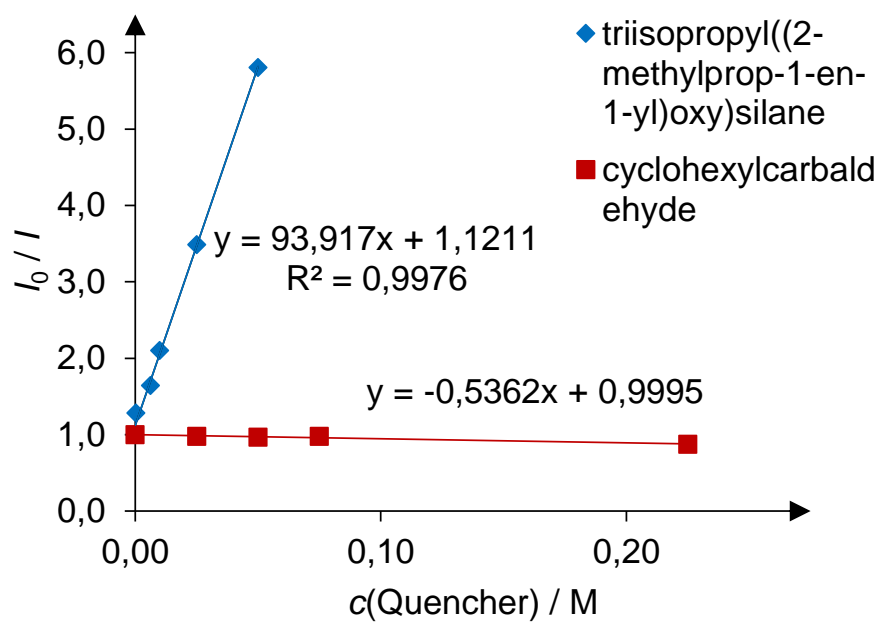

Figure S7: Stern-Volmer luminescence quenching analysis using $\left[\operatorname{Ir}\left(\mathrm{dF}\left(\mathrm{CF}_{3}\right) \mathrm{ppy}\right)_{2}\left(5,5^{\prime}-\mathrm{dCF}_{3} \mathrm{bpy}\right) \mathrm{PF}_{6}\right.$.

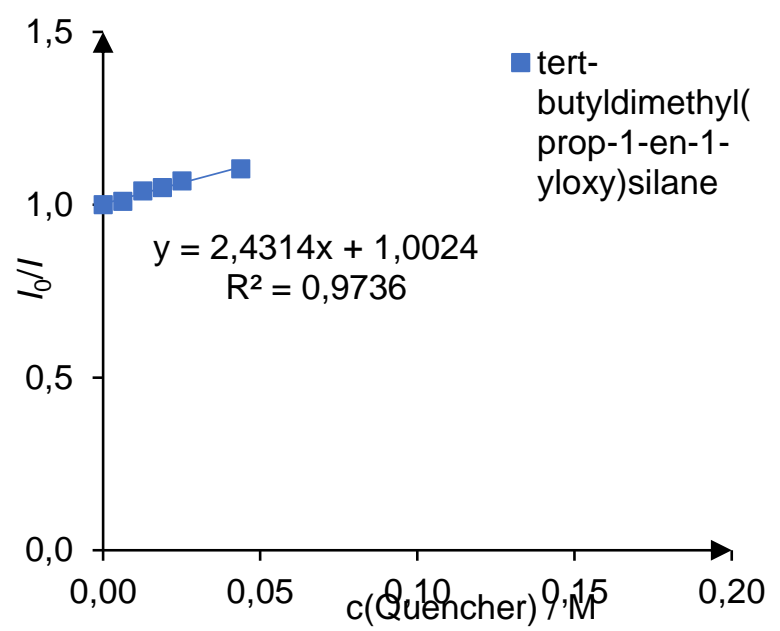

Figure S8: Stern-Volmer luminescence quenching analysis using $\left[\operatorname{Ir}\left(\mathrm{dF}\left(\mathrm{CF}_{3}\right)_{p p y}\right)_{2}\left(5,5^{\prime}-\mathrm{dCF}_{3} \mathrm{bpy}\right)\right]_{\mathrm{PF}} \mathrm{F}_{6}$

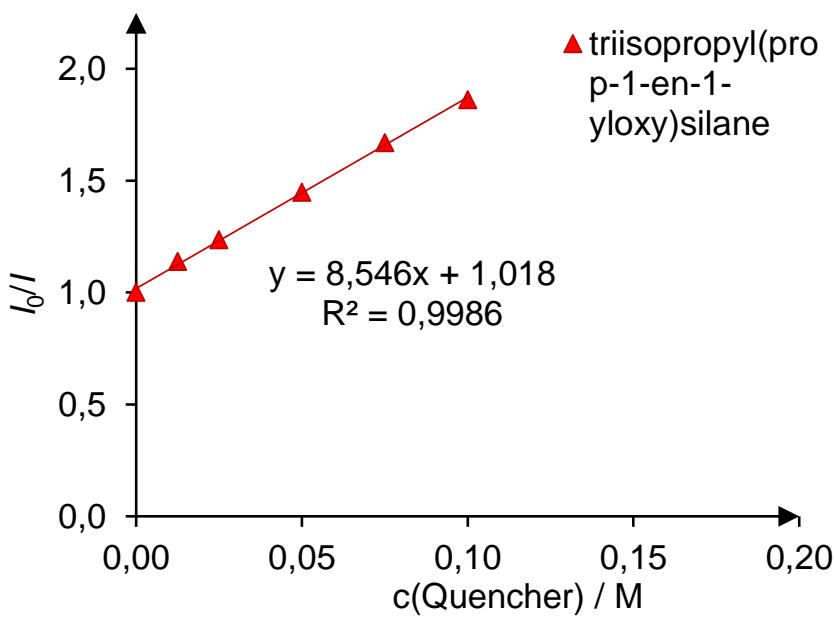

Figure S9: Stern-Volmer luminescence quenching analysis using $\left[\operatorname{Ir}\left(\mathrm{dF}\left(\mathrm{CF}_{3}\right) \mathrm{ppy}\right)_{2}\left(5,5^{\prime}-\mathrm{dCF}_{3} \mathrm{bpy}\right) \mathrm{PF}_{6}\right.$. 


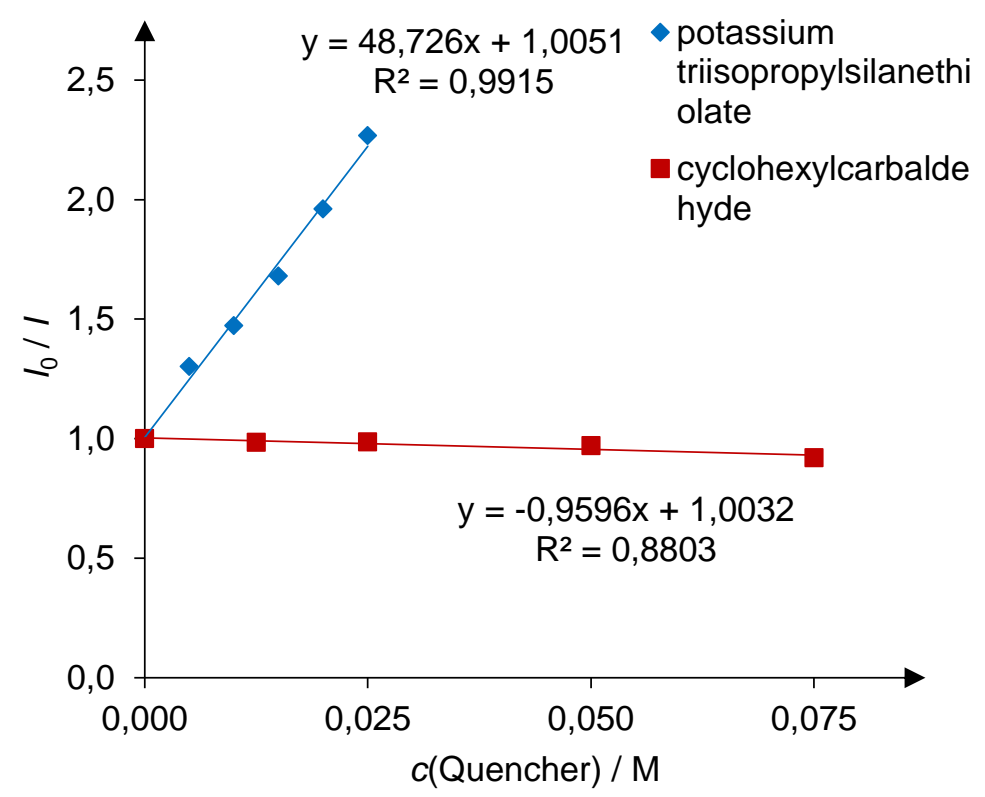

Figure S10: Stern-Volmer luminescence quenching analysis using $\left[\operatorname{Ir}(\mathrm{ppy})_{2}(\mathrm{dtbbpy})\right] \mathrm{PF} 6$.

\section{Sensitivity Assessment}

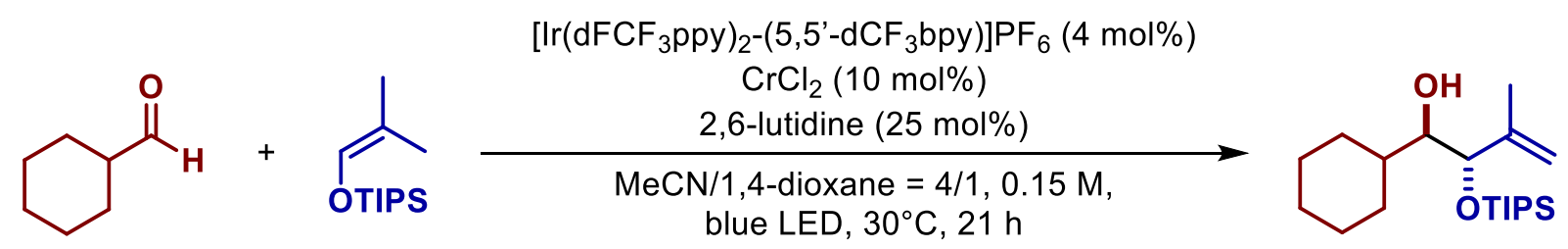

The sensitivity assessment was conducted following a modified literature procedure. ${ }^{15}$ The influence of water, oxygen, temperature and the concentration was investigated, as well as the scalability. While one parameter was varied, all other parameters were kept constant. The yield was determined via GC-FID after single-point calibration. The respective deviation in yield was calculated in reference to a control reaction ( $98 \%$ GC-FID yield on $0.1 \mathrm{mmol}$ scale).

The results are shown in table S1 together with the respective diagram in Figure S11. The reaction showed to be overall very insensitive towards most parameter changes. The addition of oxygen had a slightly negative effect, but the reaction worked well under inert atmosphere, even without degassing. Variation in concentration, temperature and light intensity were shown to be tolerated very well. Only the addition of water almost completely inhibited product formation. In summary, to reproduce the reaction, working under inert atmosphere and careful exclusion of water are the most crucial parameters while the reaction is very insensitive otherwise. As many aromatic aldehydes are known to be sensitive towards oxygen, we nevertheless recommend to degas the reaction mixture by freeze/pump/thaw cycles. 
Table S1: Results from the sensitivity assessment.

Parameter

Variation

Deviation from

control yield

Water

Concentration

Oxygen

Temperature

Intensity

Big Scale

$\begin{array}{cc}+\mathrm{H}_{2} \mathrm{O}, \mathrm{V}_{\mathrm{H} 2 \mathrm{O}}=1 \% \mathrm{~V}_{\mathrm{rxn}} & -89 \% \\ \mathrm{~V}_{\mathrm{r} \times n}-10 \% \mathrm{~V}_{\mathrm{r} \times n} & +2 \% \\ \mathrm{~V}_{\mathrm{r} \times n}+10 \% \mathrm{~V}_{\mathrm{rxn}} & -2 \% \\ \text { Inert Atmosphere } & -1 \% \\ + \text { air, } \mathrm{V}_{\mathrm{air}}=10 * \mathrm{~V}_{\mathrm{r} \times n} & -18 \% \\ T-10^{\circ} \mathrm{C} & +9 \% \\ T+10^{\circ} \mathrm{C} & -3 \% \\ I / 16 & -4 \% \\ I^{*} 16 & -1 \% \\ n * 20 & 0 \%\end{array}$

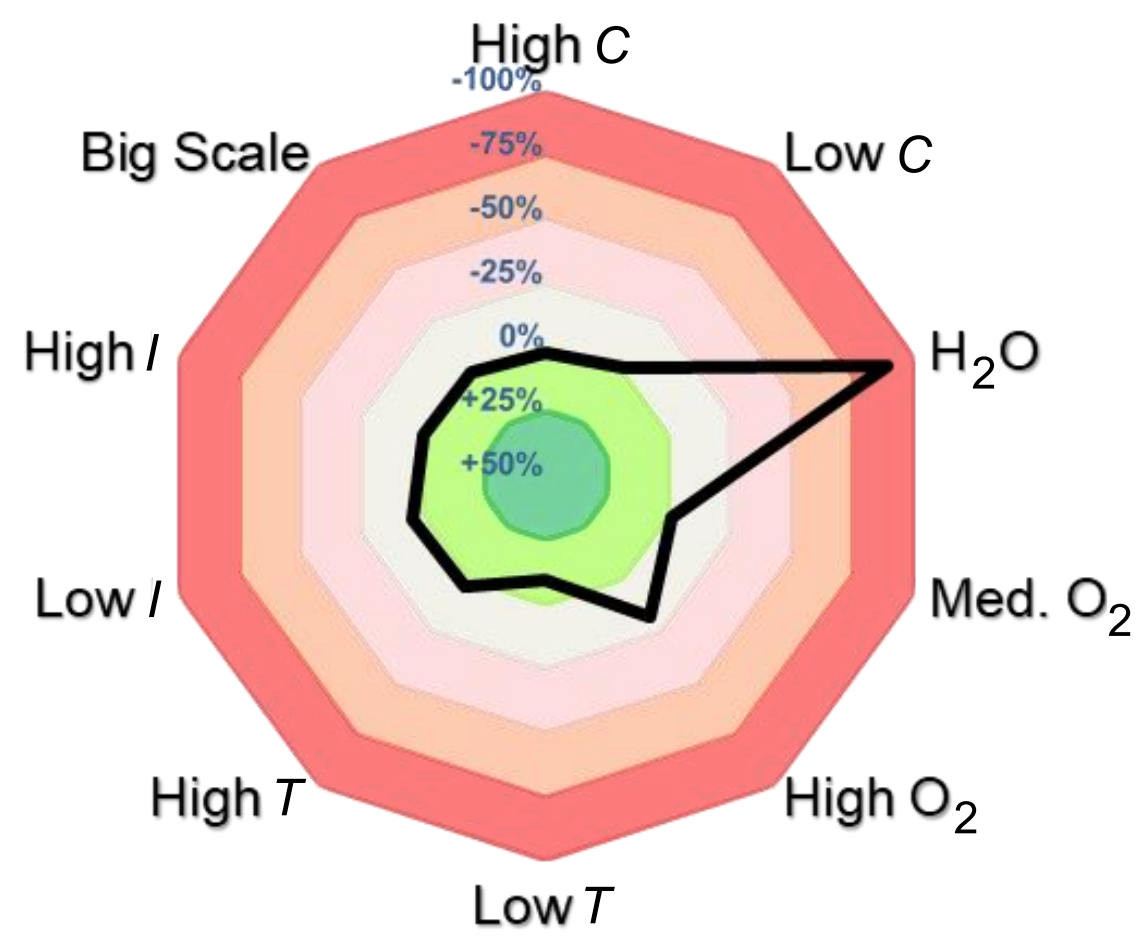

Figure S11: Radar Diagram highlighting the reaction's sensitivity. 


\section{Robustness Screen}

The robustness screen was performed following a literature procedure. ${ }^{16}$<smiles>CC(C)=C[OH+]C=CC1CCCCC1</smiles>
$\left[\operatorname{lr}\left(\mathrm{dFCF}_{3} \mathrm{ppy}_{2}-\left(5,5^{\prime}-\mathrm{dCF}_{3} \mathrm{bpy}\right)\right] \mathrm{PF}_{6}(4 \mathrm{~mol} \%)\right.$

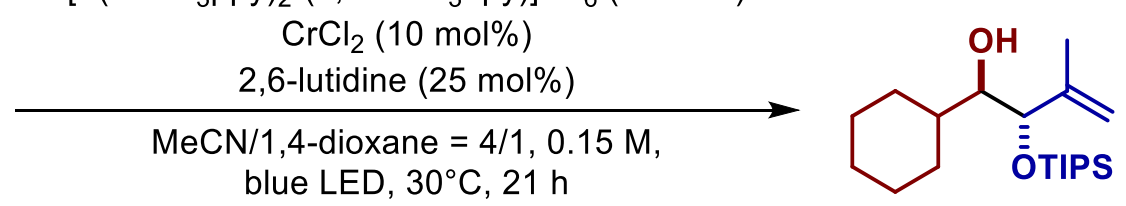

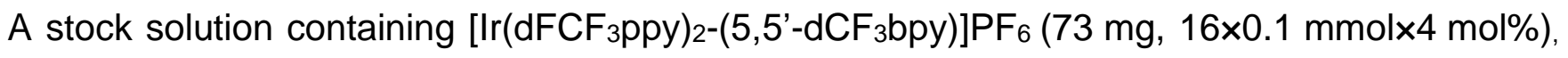
triisopropyl((2-methylprop-1-en-1-yl)oxy)silane $(1.10 \mathrm{~g}, 16 \times 0.1 \mathrm{mmol} \times 3$ equiv. $), 2,6$-lutidine $(46.5 \mu \mathrm{L}, 16 \times 0.1 \mathrm{mmol} \times 25 \mathrm{~mol} \%)$, cyclohexanecarbaldehyde $(194 \mu \mathrm{L}, 16 \times 0.1 \mathrm{mmol}), \mathrm{MeCN}$ $(8.64 \mathrm{~mL}, 16 \times 0.54 \mathrm{~mL})$ and 1,4-dioxane $(2.24 \mathrm{~mL}, 16 \times 0.14 \mathrm{~mL})$ was prepared and carefully degassed by three freeze/pump/thaw cycles. 15 oven-dried $10 \mathrm{~mL}$ reaction tubes were prepared and $\mathrm{CrCl}_{2}$ was respectively added to each reaction tube in an argon filled glovebox (solubility reasons). To each reaction tube were added one equivalent of the stock solution as well as the respective additive $(0.1 \mathrm{mmol})$. One reaction was conducted without additive as the control reaction. The reaction mixtures were irradiated with blue LEDs for $21 \mathrm{~h}$. Mesitylene was added as an internal standard and yields as well as the remaining amounts of the respective additive were determined by GC-FID analysis. The color code for the recovered additives are defined as: Recovered additive: green (>66\%), yellow (33-66\%), red $(<33 \%)$.

The color code for the product yield is the same, but referring to the yield of the control reaction (84\%), considering the special setup and volumina effects when preparing the stock solution, leading to: Product yields: $\quad$ green (>56\%), yellow $(28-56 \%)$, red $(<28 \%)$.

The respective product yields and amounts of additives recovered are shown in Figures S12 and S13:

Product Yields

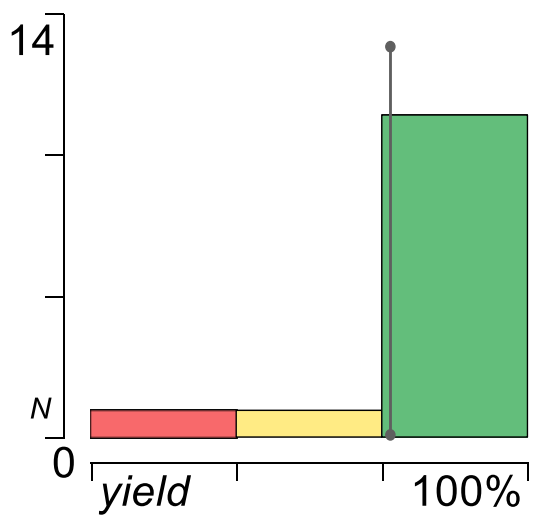

Recovered Additives

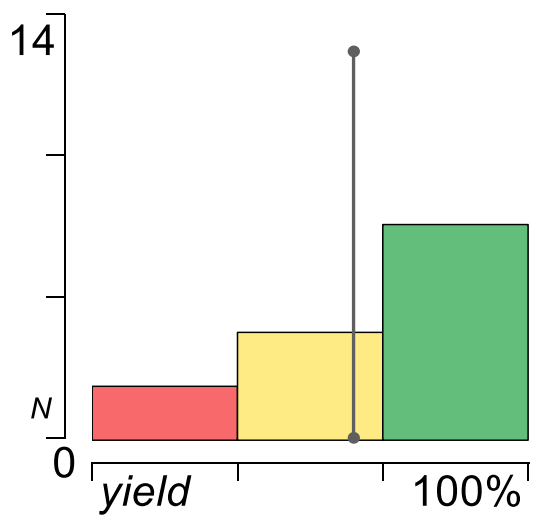

Figure S12: Graphical overview over the results of the "robustness screening". 


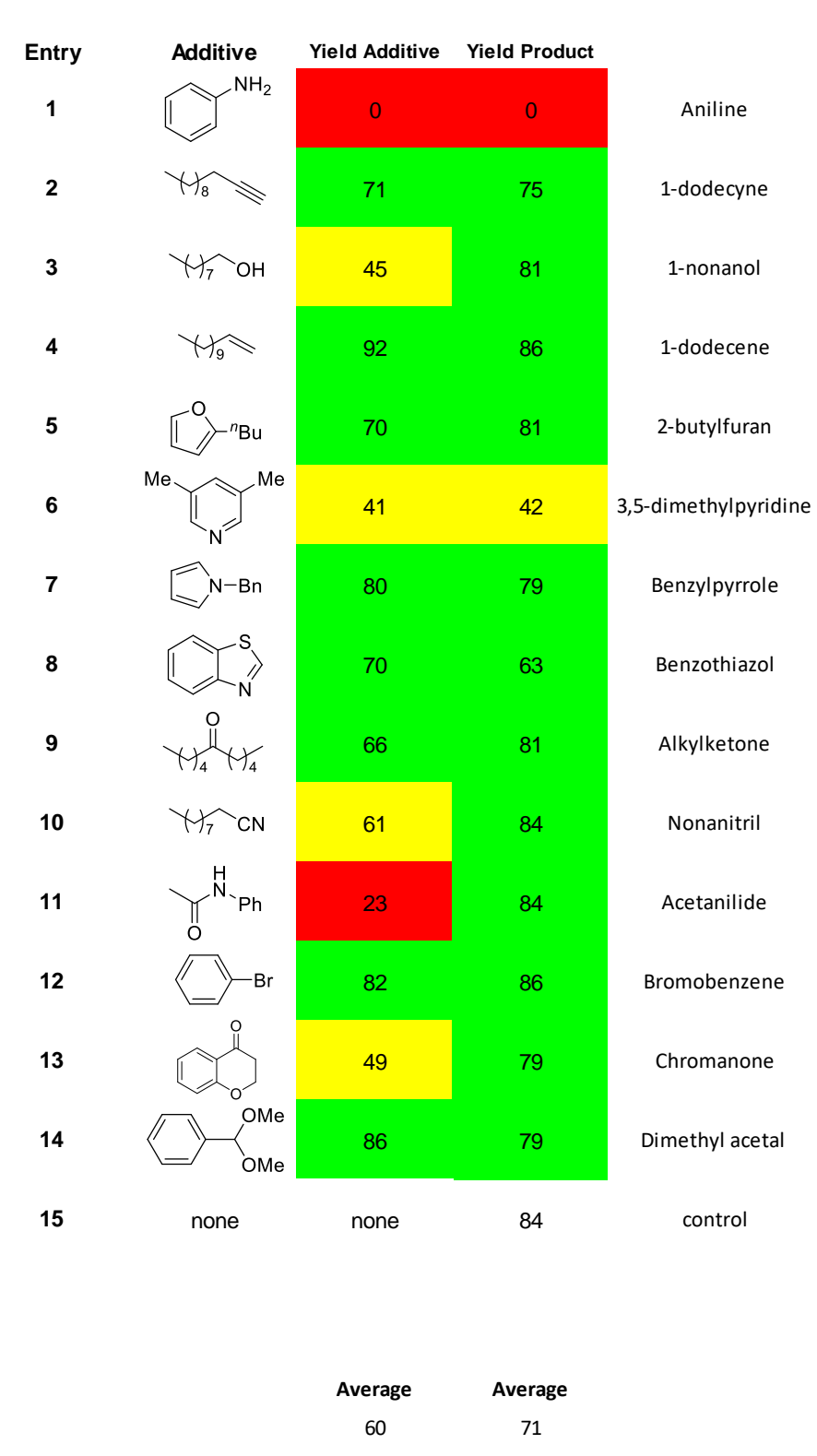

Figure S13: Results of the "robustness screening".

\section{NOE Experiment}

To assign the major/ minor diastereomers to the anti or syn configuration, we conducted the following NOE experiment. The cyclization of the syn diasteromer should form a rigid fivemembered ring with the two hydrogen atoms in anti configuration, the anti diastereomer should show the respective syn configuration, leading to a detectable NOE signal (Figure S14). 
<smiles>C=CC(O)C(O)[GeH3]</smiles>

syn, minor<smiles>C=C[C@H](O)C([GeH3])[GeH3]</smiles>

anti, major
1) TBAF (1.2 equiv.) THF, rt, overnight

2) triphosgene (1.1 equiv.) pyridine (5.0 equiv.)

$\mathrm{Ch}_{2} \mathrm{Cl}_{2}, 0^{\circ} \mathrm{C}, 2 \mathrm{~h}$<smiles>[X][N+](=O)[O-]</smiles>

Figure S14: Determination of the relative configuration of the diastereomers via a NOE experiment.

The NOE experiment was performed for both diastereomers and only for the major one, a positive NOE could be observed (Figure S15). This is in accordance with the observed configuration in the obtained crystal structure.

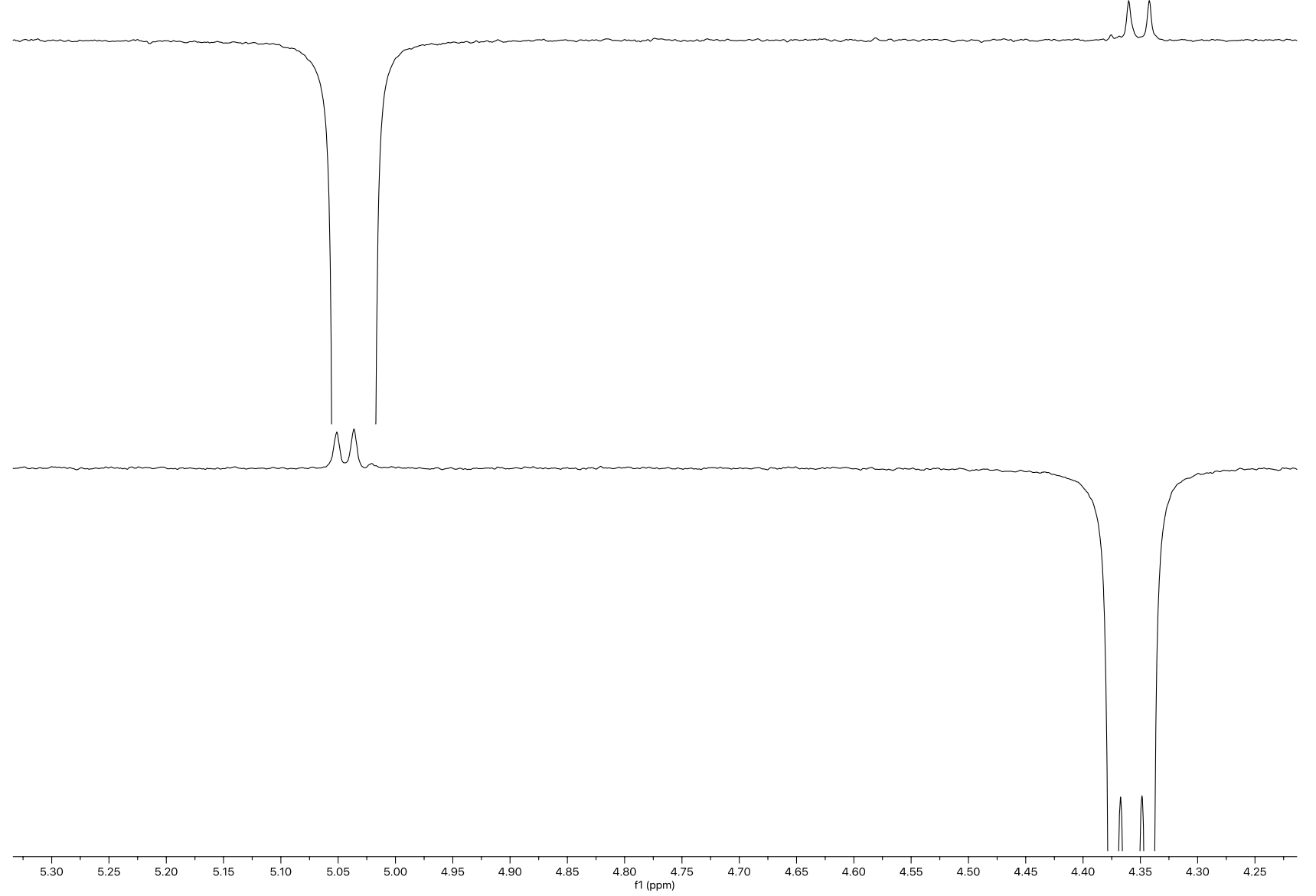

Figure S15: NOE experiment of the major diastereomer. Negative intensities show proton spins that were saturated by RF field irradiation. Positive intensities show spin coupling. 


\section{Limitations}<smiles>CC(Br)=C[Se-]</smiles><smiles>C/C(=C\O[Si](C)(C(C)C)C(C)C)[Si](C)(C(C)C)C(C)C</smiles><smiles>C/C=C/OC1CCCCO1</smiles><smiles>C/C=C/OCOCC</smiles><smiles>[SeH2]OC1=CCCCC1</smiles><smiles>CC=CC=C[O+]S(=O)(=O)c1ccccc1</smiles><smiles>CC=COC(C)=O</smiles><smiles>C1=COCCC1</smiles><smiles>O=Cc1c[nH]c2ccccc12</smiles><smiles>CC(C)(C)C=O</smiles>

Figure S16: Unreactive starting materials.

The starting materials shown in Figure S16 were no suitable coupling partners.

\section{Selected Optimization Results}<smiles>C=C[C@H](O[Sb])C(O)C1CCCCC1</smiles>

Table S2: Selected optimization results, using three equivalents of the silyl enol ether.

\begin{tabular}{|c|c|c|c|}
\hline $\begin{array}{l}\text { Solvent } \\
\text { (Ratio) }\end{array}$ & Photocatalyst (Loading) & Base (Loading) & Yield \\
\hline $\mathrm{MeCN}$ & $\begin{array}{l}{\left[\operatorname{Ir}\left(\mathrm{dF}\left(\mathrm{CF}_{3}\right) \mathrm{ppy}\right)_{2}\left(5,5^{\prime}-\right.\right.} \\
\left.\left.\mathrm{dCF}_{3} \mathrm{bpy}\right)\right] \mathrm{PF}_{6}(10 \mathrm{~mol} \%)\end{array}$ & Collidine (25 mol\%) & $77 \%$ \\
\hline $\mathrm{MeCN}$ & $\begin{array}{l}{\left[\operatorname{Ir}\left(\mathrm{dF}\left(\mathrm{CF}_{3}\right) \mathrm{ppy}\right)_{2}\left(4,4{ }^{\prime}-\right.\right.} \\
\left.\left.\mathrm{dCF}_{3} \mathrm{bpy}\right)\right] \mathrm{PF}_{6}(10 \mathrm{~mol} \%)\end{array}$ & Collidine (25 mol\%) & $19 \%$ \\
\hline $\mathrm{MeCN}$ & $\begin{array}{l}{\left[\operatorname{lr}\left(\mathrm{dF}\left(\mathrm{CF}_{3}\right) \mathrm{ppy}\right)_{2}\left(4,4^{\prime}-\right.\right.} \\
\mathrm{dFbpy} \mathrm{PF}_{6}(10 \mathrm{~mol} \%)\end{array}$ & Collidine (25 mol\%) & $38 \%$ \\
\hline $\mathrm{MeCN}$ & $\begin{array}{l}\text { 9-Mesityl-10- } \\
\text { methylacridinium } \\
\text { tetrafluoroborate (10 } \mathrm{mol} \%)\end{array}$ & Collidine (25 mol\%) & trace \\
\hline $\mathrm{CH}_{2} \mathrm{Cl}_{2}$ & $5,5^{\prime} \mathrm{CF}_{3}(10 \mathrm{~mol} \%)$ & Collidine (25 mol\%) & - \\
\hline DME & $5,5^{\prime} \mathrm{CF}_{3}(10 \mathrm{~mol} \%)$ & Collidine (25 mol\%) & trace \\
\hline DMA & $5,5^{\prime} \mathrm{CF}_{3}(10 \mathrm{~mol} \%)$ & Collidine (25 mol\%) & trace \\
\hline DMF & $5,5^{\prime} \mathrm{CF}_{3}(10 \mathrm{~mol} \%)$ & Collidine (25 mol\%) & - \\
\hline THF & $5,5^{\prime} \mathrm{CF}_{3}(10 \mathrm{~mol} \%)$ & Collidine (25 mol\%) & - \\
\hline
\end{tabular}




\begin{tabular}{|c|c|c|c|}
\hline $\mathrm{MeCN}$ & $5,5^{\prime} \mathrm{CF}_{3}(4 \mathrm{~mol} \%)$ & Pyridine (25mol\%) & - \\
\hline $\mathrm{MeCN}$ & $5,5^{\prime} \mathrm{CF}_{3}(4 \mathrm{~mol} \%)$ & $\mathrm{Li}_{2} \mathrm{CO}_{3}(25 \mathrm{~mol} \%)$ & - \\
\hline $\mathrm{MeCN}$ & $5,5^{\prime} \mathrm{CF}_{3}(4 \mathrm{~mol} \%)$ & Lutidine (25mol\%) & $66 \%$ \\
\hline $\mathrm{MeCN}$ & $5,5^{\prime} \mathrm{CF}_{3}(4 \mathrm{~mol} \%)$ & Collidine (25mol\%) & $57 \%$ \\
\hline $\mathrm{MeCN}$ & $5,5^{\prime} \mathrm{CF}_{3}(4 \mathrm{~mol} \%)$ & Quinuclidine (25mol\%) & - \\
\hline $\begin{array}{l}\text { MeCN/DCM } \\
(4 / 1)\end{array}$ & $5,5^{\prime} \mathrm{CF}_{3}$ (4 $\left.\mathrm{mol} \%\right)$ & Collidine (25 mol\%) & $60 \%$ \\
\hline $\begin{array}{l}\text { MeCN/DME } \\
(4 / 1)\end{array}$ & $5,5^{\prime} \mathrm{CF}_{3}(4 \mathrm{~mol} \%)$ & Collidine (25 mol\%) & $55 \%$ \\
\hline $\begin{array}{l}\mathrm{MeCN} / \mathrm{THF} \\
(4 / 1)\end{array}$ & $5,5^{\prime} \mathrm{CF}_{3}(4 \mathrm{~mol} \%)$ & Collidine (25 mol\%) & $29 \%$ \\
\hline $\begin{array}{l}\text { MeCN/Diox } \\
(4 / 1)\end{array}$ & $5,5^{\prime} \mathrm{CF}_{3}(4 \mathrm{~mol} \%)$ & Collidine (25 mol\%) & $74 \%$ \\
\hline $\begin{array}{l}\mathrm{MeCN} / \mathrm{Et}_{2} \mathrm{O} \\
(4 / 1)\end{array}$ & $5,5^{\prime} \mathrm{CF}_{3}(4 \mathrm{~mol} \%)$ & Collidine (25 mol\%) & $53 \%$ \\
\hline
\end{tabular}

\section{Comment on the difference between Fukuzumi-type and Iridium-based catalysts:}

Our empirical experience showed that the Fukuzumi-type acridinium catalyst performs extremely well in Chromium/photoredox dual catalytic systems when using unfunctionalized alkenes or styrenes, while the Iridium-based catalysts are often needed when functional groups are present, such as silyl alkenyl ethers or amines.

In addition, we observed that the Fukuzumi-type catalyst did not tolerate most of the heteroaromatic aldehydes we tried, while the Iridium-based catalyst could functionalize them.

We propose two reasons for this different reactivity:

1) Productive vs. non-productive decomposition pathways of the single-electron-oxidized alkene: Takai showed, that unfunctionalized alkenes in very high excess (20 equiv.) can function as allyl radical precursors even without a base being present. In this case, the single-electron-oxidized species is highly acidic and the only major decomposition pathway is the productive deprotonation to give the stabilized allylic radical. As unfunctionalized alkenes are very hard to directly oxidize, the high excess is needed, to render the number of generated allyl radicals high enough to sufficiently functionalize the respective aldehyde, with no major side reactions taking place.

In our case, when using silyl enol ethers ( 3 equiv.) we observed a huge number of silyl containing decomposition products, if the single-electron-oxidized silyl ether is formed either too quickly and/ or is not directly intercepted by a suitable base (see the long reaction times as well as the crucial role of lutidine). This can explain why the Fukuzumi-type catalyst performs worse, as it is a much stronger oxidant and can enhance the fast, non-productive decomposition pathways instead of the productive deprotonation, even in presence of a base.

2) The lower stability of organic dyes in general might play a crucial role as well. The presence of nucleophilic silyl enol ethers, amines or other functional groups in large excess can lead to decomposition of the organic dye. This could also explain, why heteroaromatic aldehydes often seem to be not compatible with the Fukuzumi-type catalyst. 


\section{Crystallographic Data}

X-Ray diffraction: Data sets for compound 2l were collected with a Bruker D8 Venture PHOTON III diffractometer. Programs used: data collection: APEX3 V2016.1-01 (Bruker AXS Inc., 2016); cell refinement: SAINT V8.37A (Bruker AXS Inc., 2015); data reduction: SAINT V8.37A (Bruker AXS Inc., 2015); absorption correction, SADABS V2014/7 (Bruker AXS Inc., 2014); structure solution SHELXT-2015²; structure refinement SHELXL-2015 and graphics, $X P^{4}$. R-values are given for observed reflections, and $w R^{2}$ values are given for all reflections.

Exceptions and special features: For compound 2l the isopropenyl group and one isopropyl group were found disordered over two positions. Several restraints (SADI, SAME, ISOR, EADP and SIMU) were used in order to improve refinement stability.

X-ray crystal structure analysis of 2I (Figure S17): A colorless prism-like specimen of $\mathrm{C}_{21} \mathrm{H}_{34} \mathrm{Br}_{2} \mathrm{O}_{4} \mathrm{Si}$, approximate dimensions $0.100 \mathrm{~mm} \times 0.160 \mathrm{~mm} \times 0.180 \mathrm{~mm}$, was used for the $X$-ray crystallographic analysis. The X-ray intensity data were measured on a Bruker APEX II diffractometer system equipped with a fine-focus sealed tube $\mathrm{Cu}$ sealed tube (CuKa, $\lambda=1.54178$ $\AA)$ and a graphite monochromator. A total of 897 frames were collected. The total exposure time was 12.45 hours. The frames were integrated with the Bruker SAINT software package using a wide-frame algorithm. The integration of the data using an orthorhombic unit cell yielded a total of 33410 reflections to a maximum $\theta$ angle of $66.64^{\circ}$ ( $0.84 \AA$ resolution), of which 4167 were independent (average redundancy 8.018, completeness $=99.8 \%, R_{\text {int }}=2.85 \%, R_{\text {sig }}=1.72 \%$ ) and $4021(96.50 \%)$ were greater than $2 \sigma\left(F^{2}\right)$. The final cell constants of $\underline{a}=12.8335(2) \AA, \underline{\mathrm{b}}=$ $14.5079(2) \AA, \underline{c}=25.3950$ (3) $\AA$, volume $=4728.22(11) \AA^{3}$, are based upon the refinement of the $X Y Z$-centroids of 9944 reflections above $20 \sigma(\mathrm{I})$ with $6.961^{\circ}<2 \theta<133.1^{\circ}$. Data were corrected for absorption effects using the Multi-Scan method (SADABS). The ratio of minimum to maximum apparent transmission was 0.845 . The calculated minimum and maximum transmission coefficients (based on crystal size) are 0.4650 and 0.6330 . The structure was

1. ${ }^{1} A P E X 3$ (2016), SAINT (2015) and SADABS (2015), Bruker AXS Inc., Madison, Wisconsin, USA.

2. Sheldrick, G. M., SHELXT - Integrated space-group and crystal-structure determination, Acta Cryst., 2015, A71, 3-8.

3. Sheldrick, G.M., Crystal structure refinement with SHELXL, Acta Cryst., 2015, C71 (1), 3-8.

4. XP-Interactive molecular graphics, Version 5.1, Bruker AXS Inc., Madison, Wisconsin, USA, 1998. 
solved and refined using the Bruker SHELXTL Software Package, using the space group Pbca, with $\mathrm{Z}=8$ for the formula unit, $\mathrm{C}_{21} \mathrm{H}_{34} \mathrm{Br}_{2} \mathrm{O}_{4} \mathrm{Si}$. The final anisotropic full-matrix least-squares refinement on $\mathrm{F}^{2}$ with 312 variables converged at $\mathrm{R} 1=2.72 \%$, for the observed data and $\mathrm{wR} 2$ $=6.75 \%$ for all data. The goodness-of-fit was 1.076 . The largest peak in the final difference electron density synthesis was $0.568 \mathrm{e}^{-} / \AA^{3}$ and the largest hole was $-1.152 \mathrm{e} / \AA^{3}$ with an RMS deviation of $0.073 \mathrm{e}^{\mathrm{e} / \AA^{3}}$. On the basis of the final model, the calculated density was $1.513 \mathrm{~g} / \mathrm{cm}^{3}$ and $F(000), 2208$ e. CCDC Nr.: 2016609.

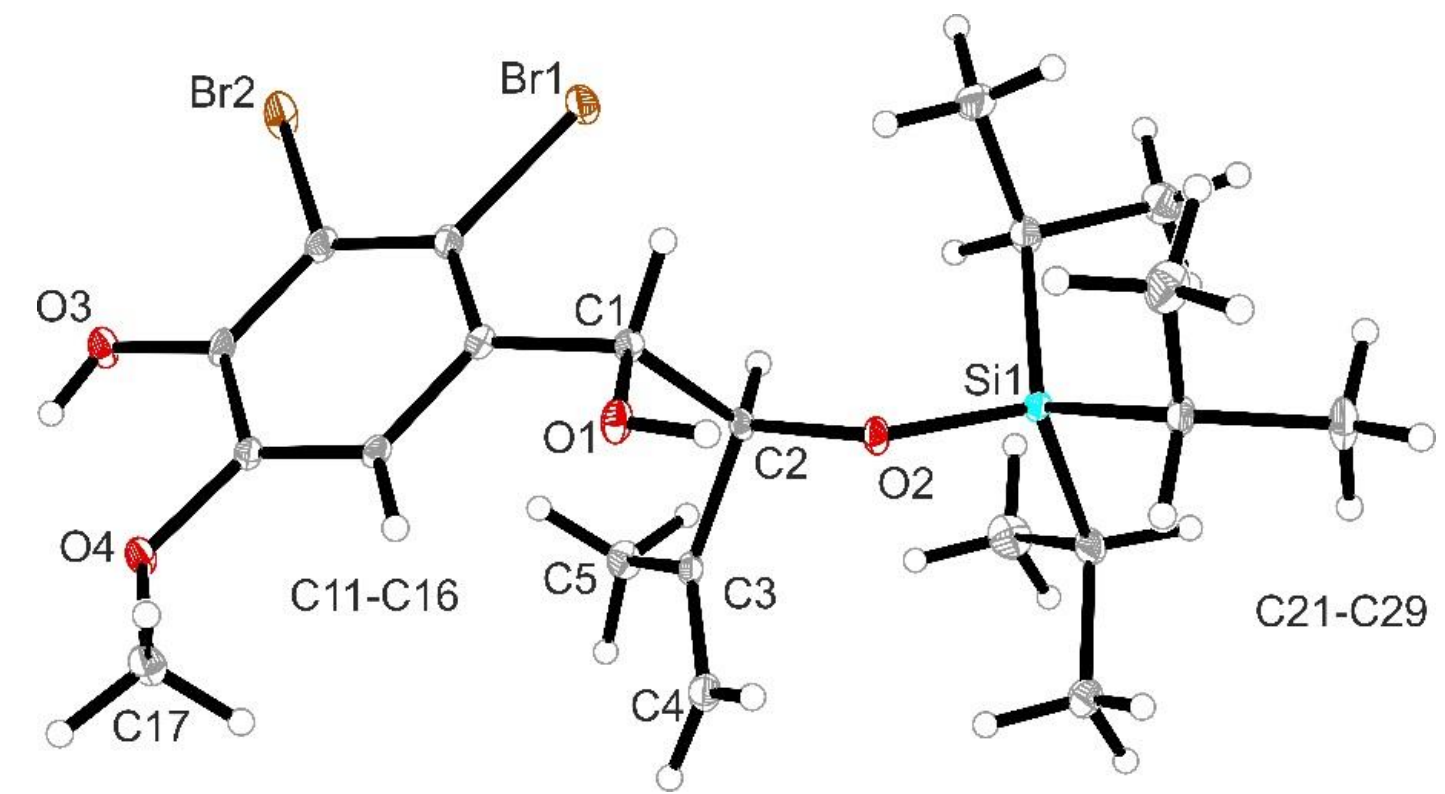

Figure S17: Crystal structure of compound 2I. Thermal ellipsoids are shown at $30 \%$ probability. 


\section{Spectra}
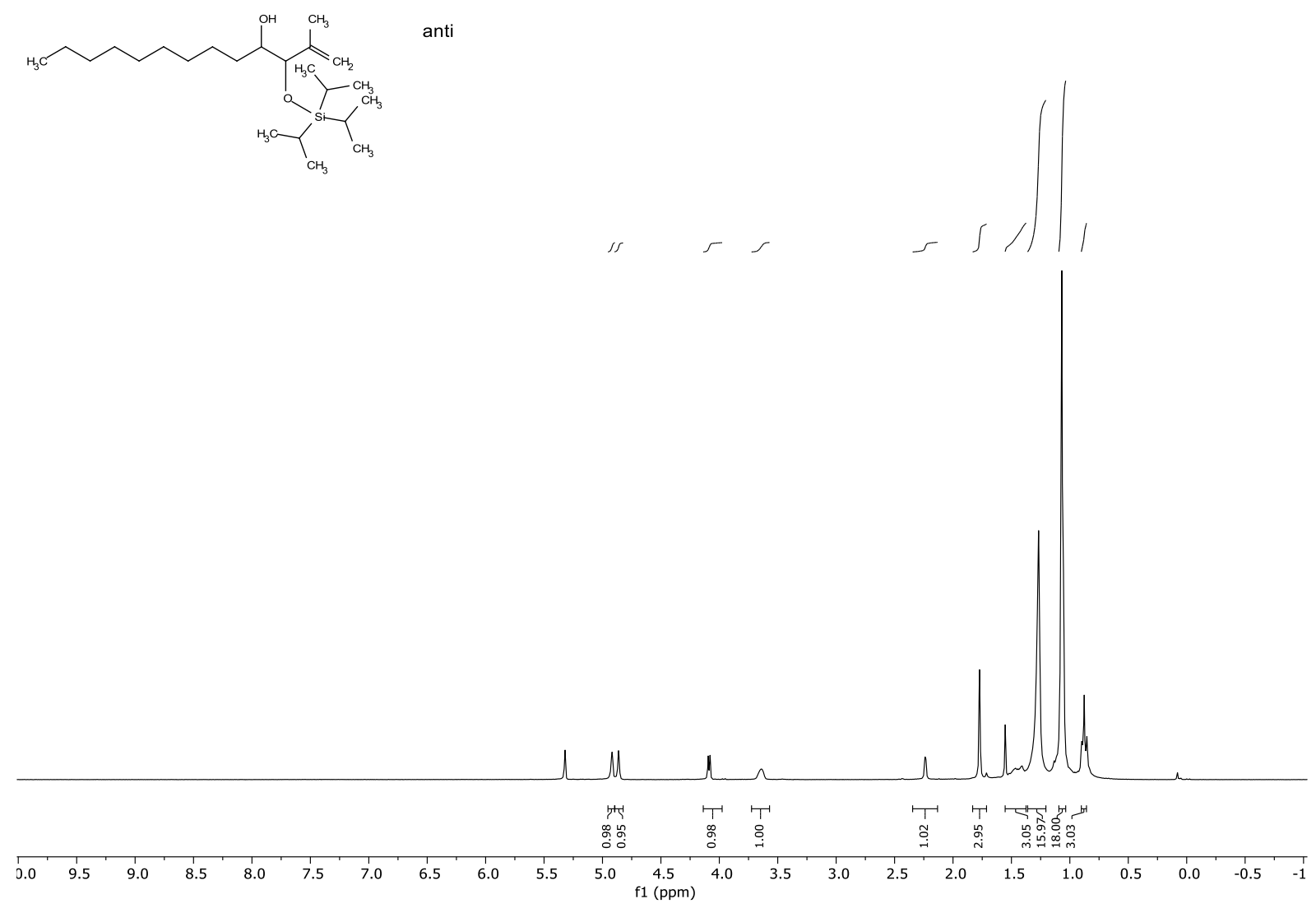

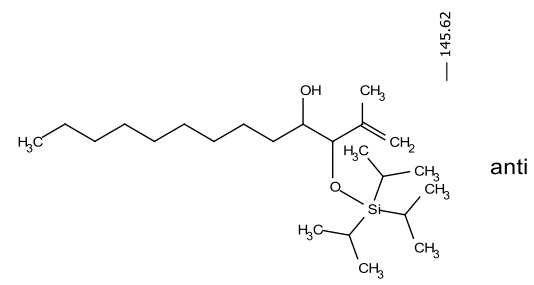

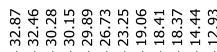

प许?

anti

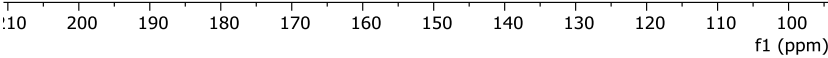

$\begin{array}{llllllllll}1 & 1 & 1 & 1 & 1 & 1 & 1 & 1 & 1 & 1 \\ & 70 & 60 & 50 & 40 & 30 & 20 & 10 & 0 & -1\end{array}$ 

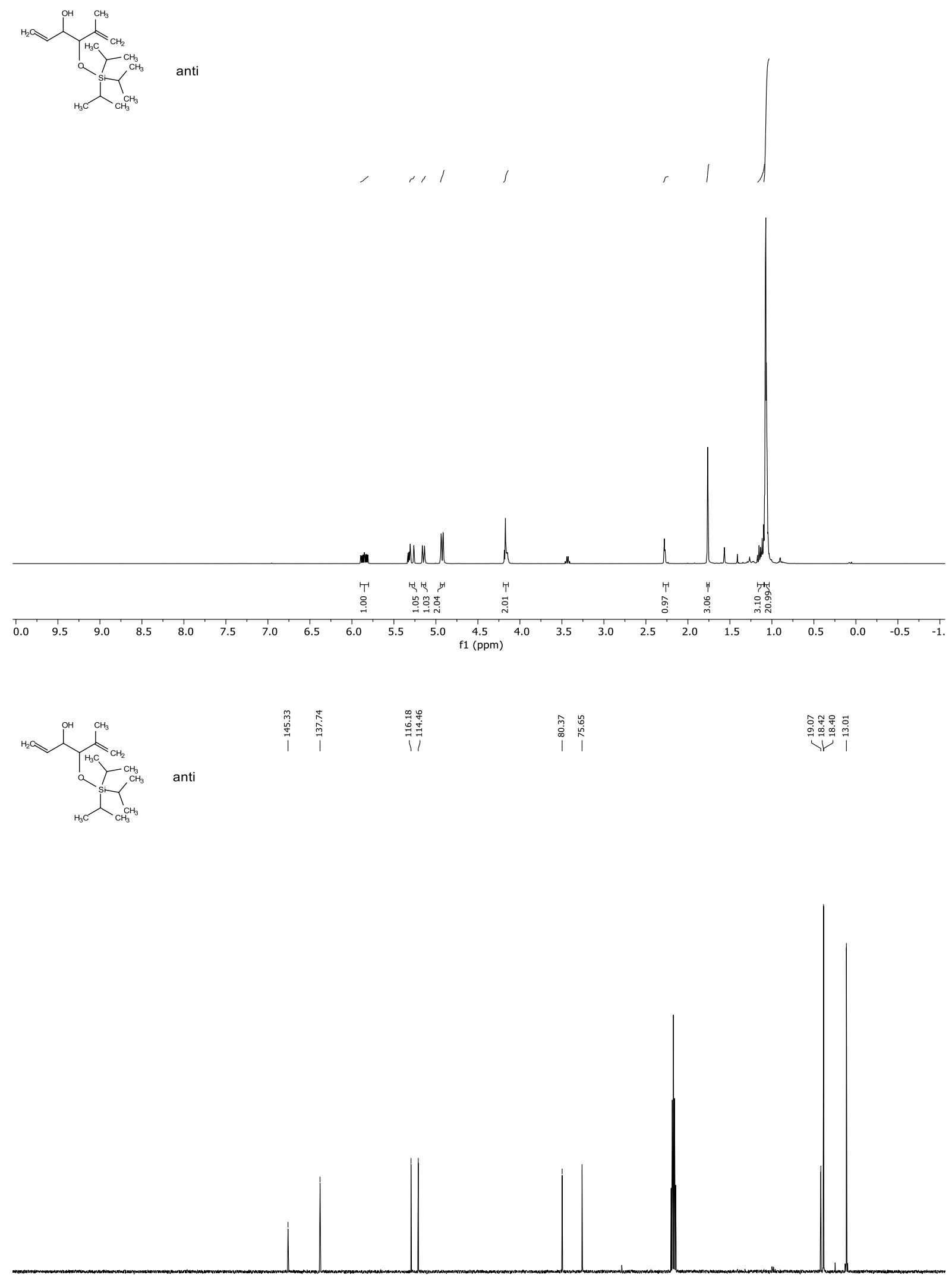

$\begin{array}{llllllllllllllllllllllllllllll}1 & 200 & 190 & 180 & 170 & 160 & 150 & 140 & 130 & 120 & 110 & 100 & 90 & 80 & 70 & 60 & 50 & 40 & 30 & 20 & 10 & 0 & -1\end{array}$ 

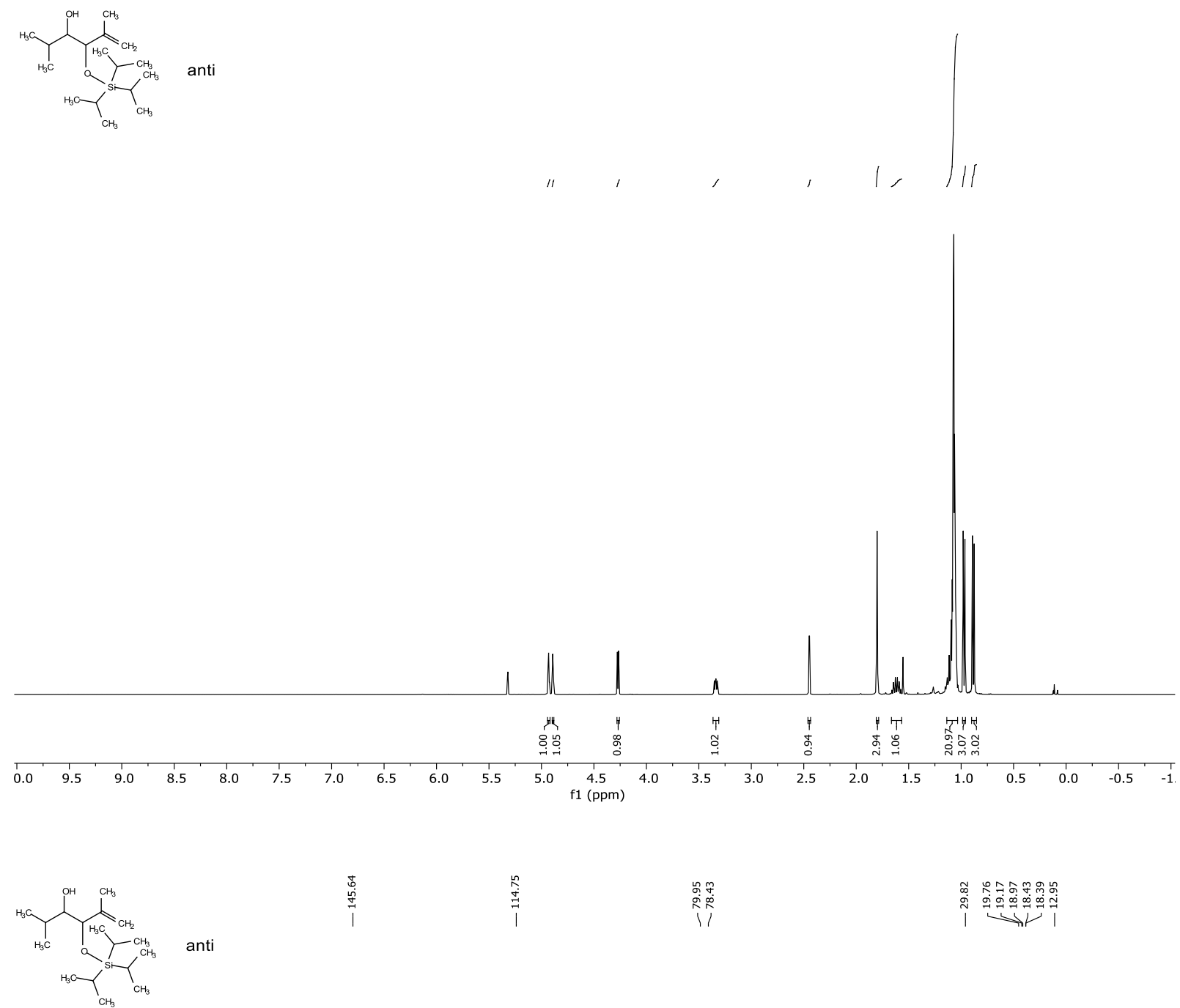

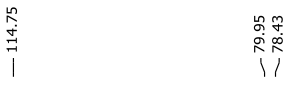

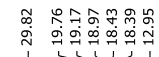

1 r

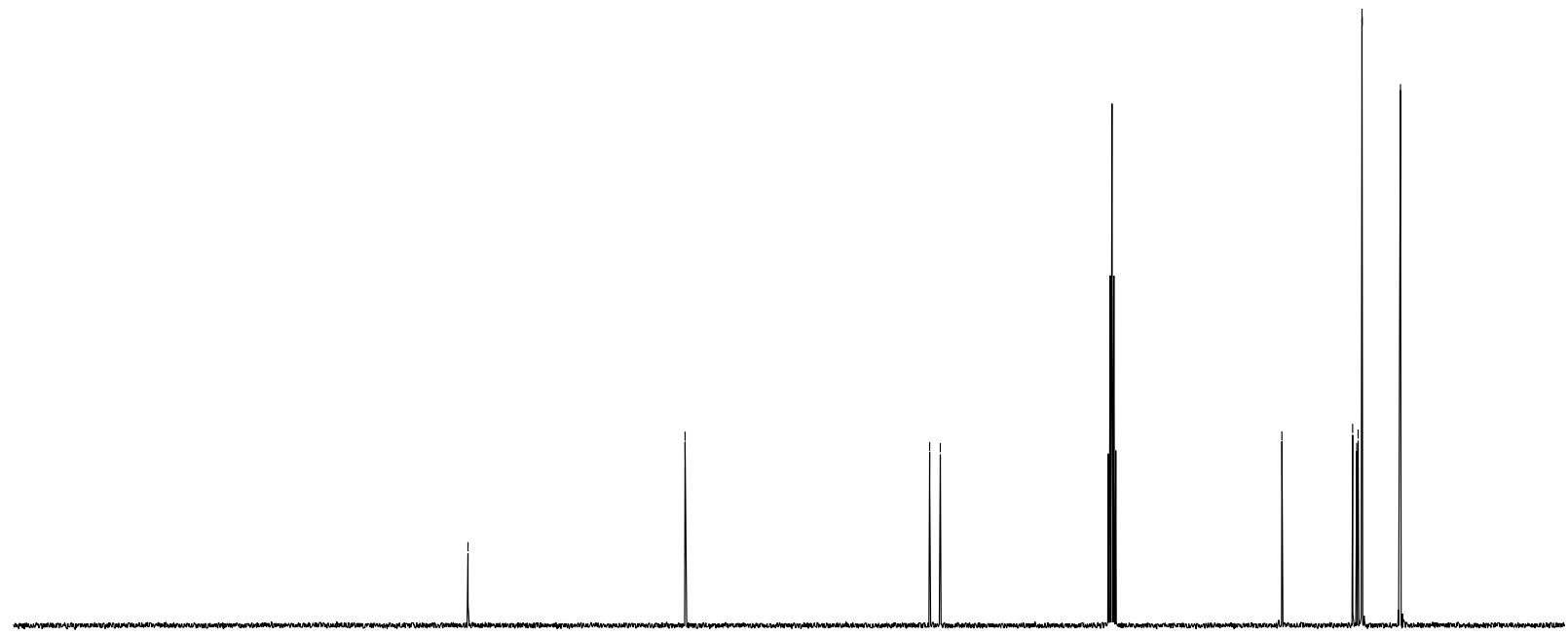

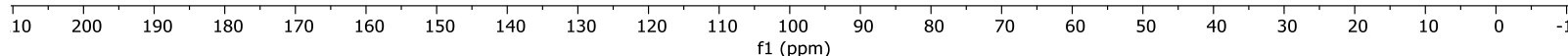



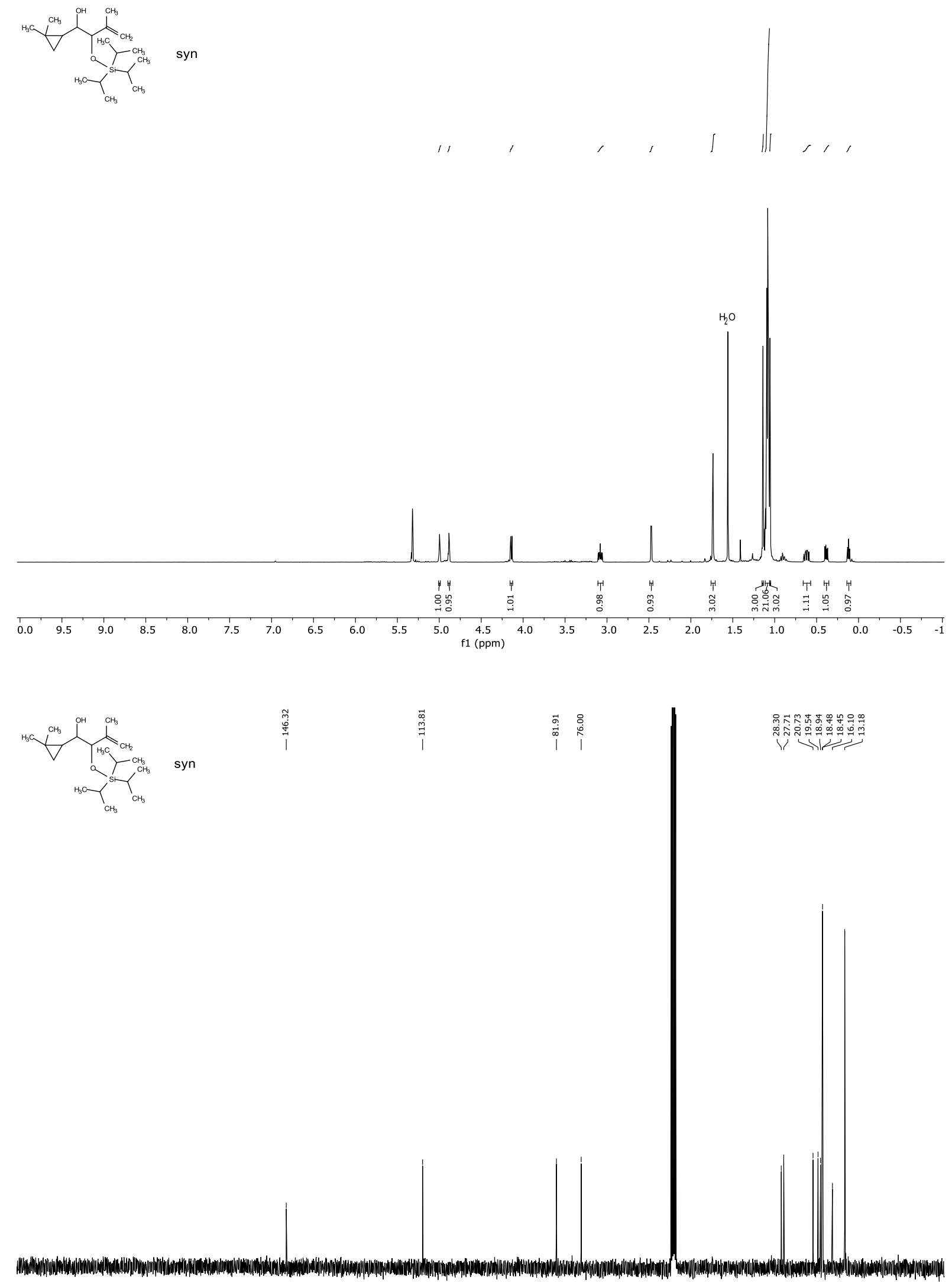

$\begin{array}{lllllllllllllllllllllllll}1 & 10 & 200 & 190 & 180 & 170 & 160 & 150 & 140 & 130 & 120 & 110 & 100 & 90 & 80 & 70 & 60 & 50 & 40 & 30 & 20 & 10 & 0 & -1\end{array}$ 


$$
\text { anti }
$$
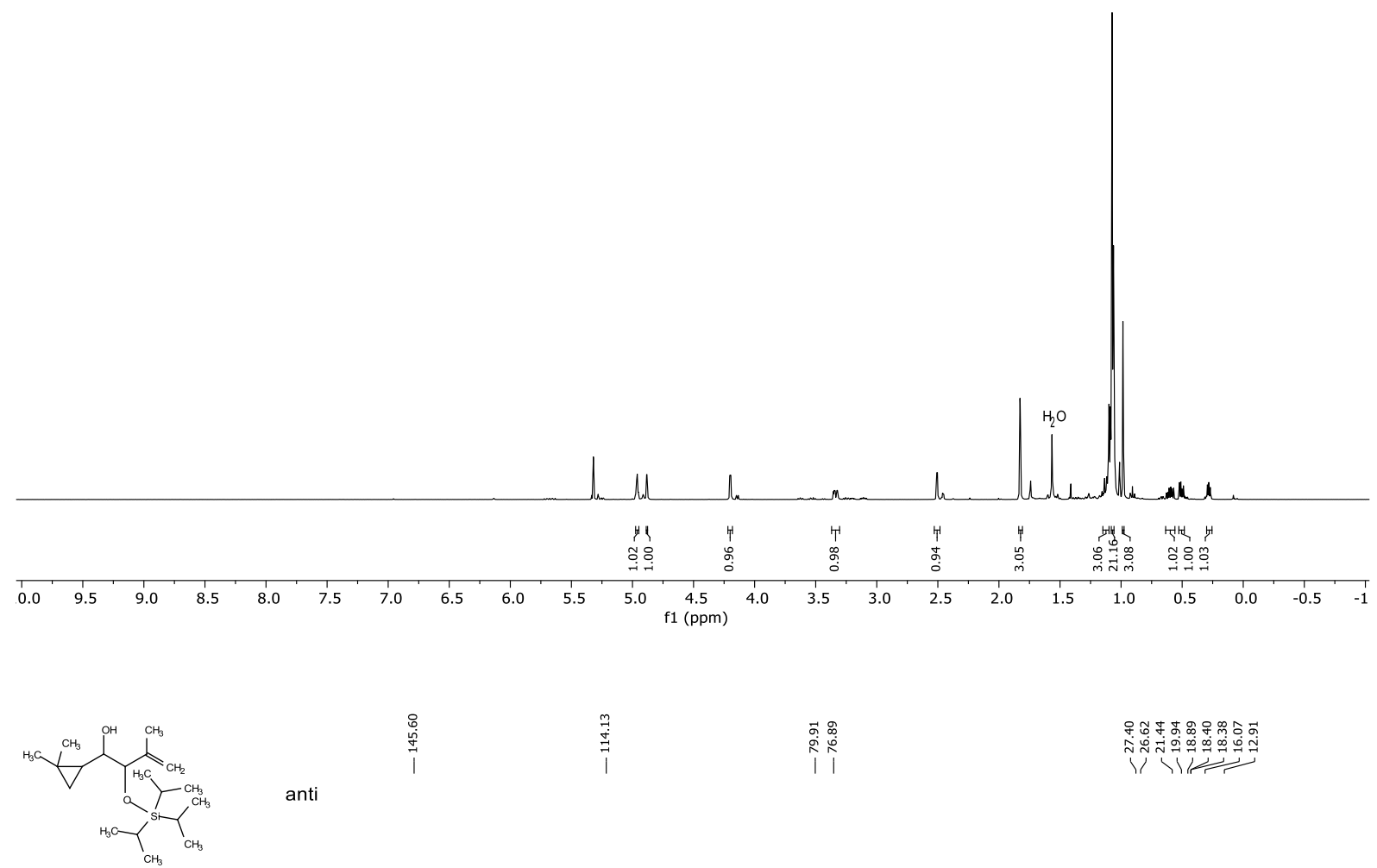

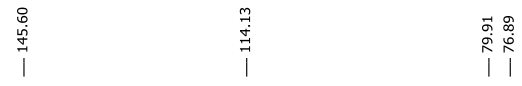

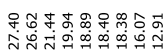

anti

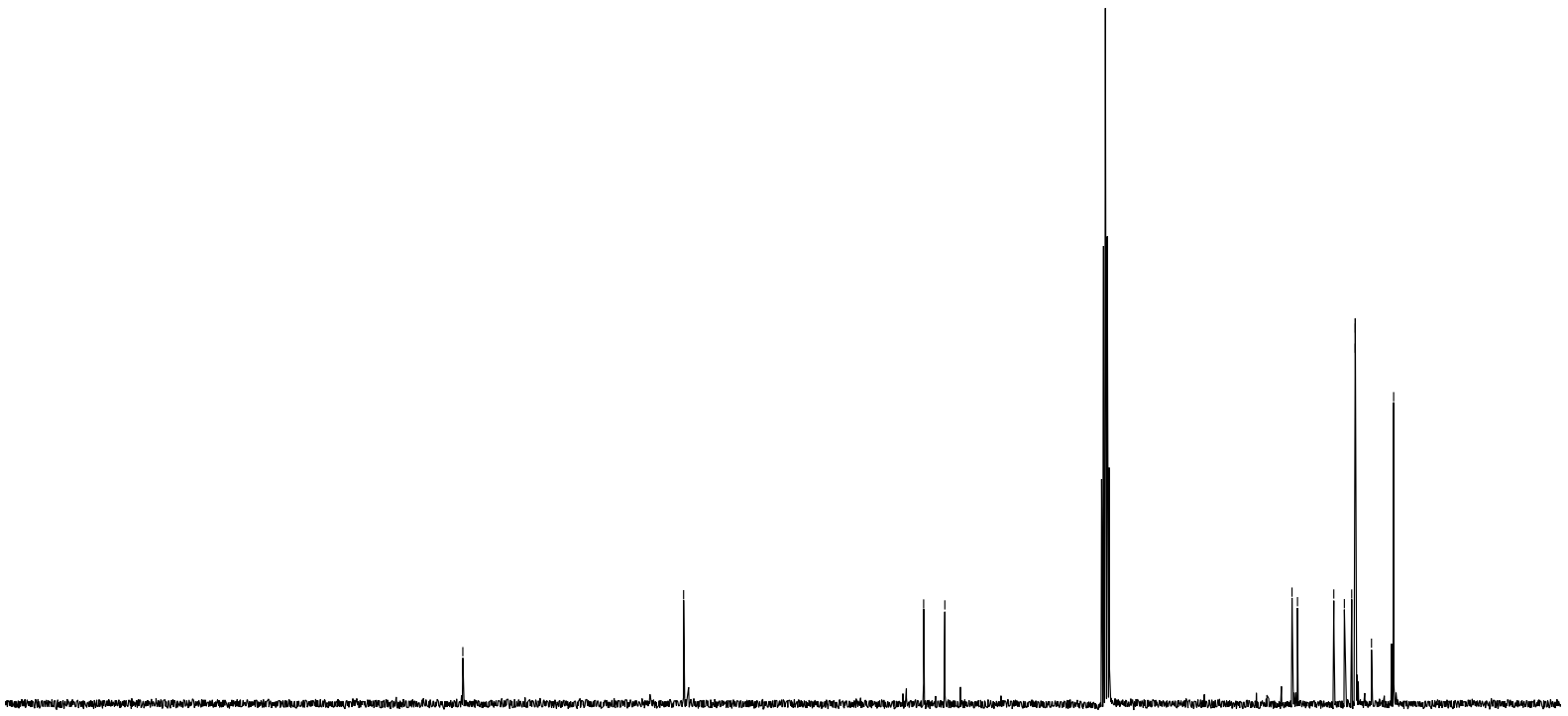

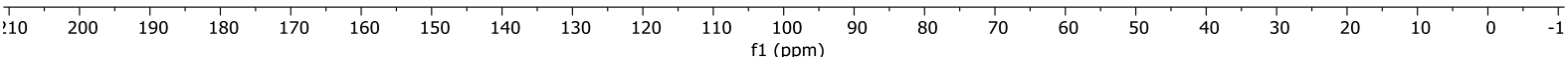



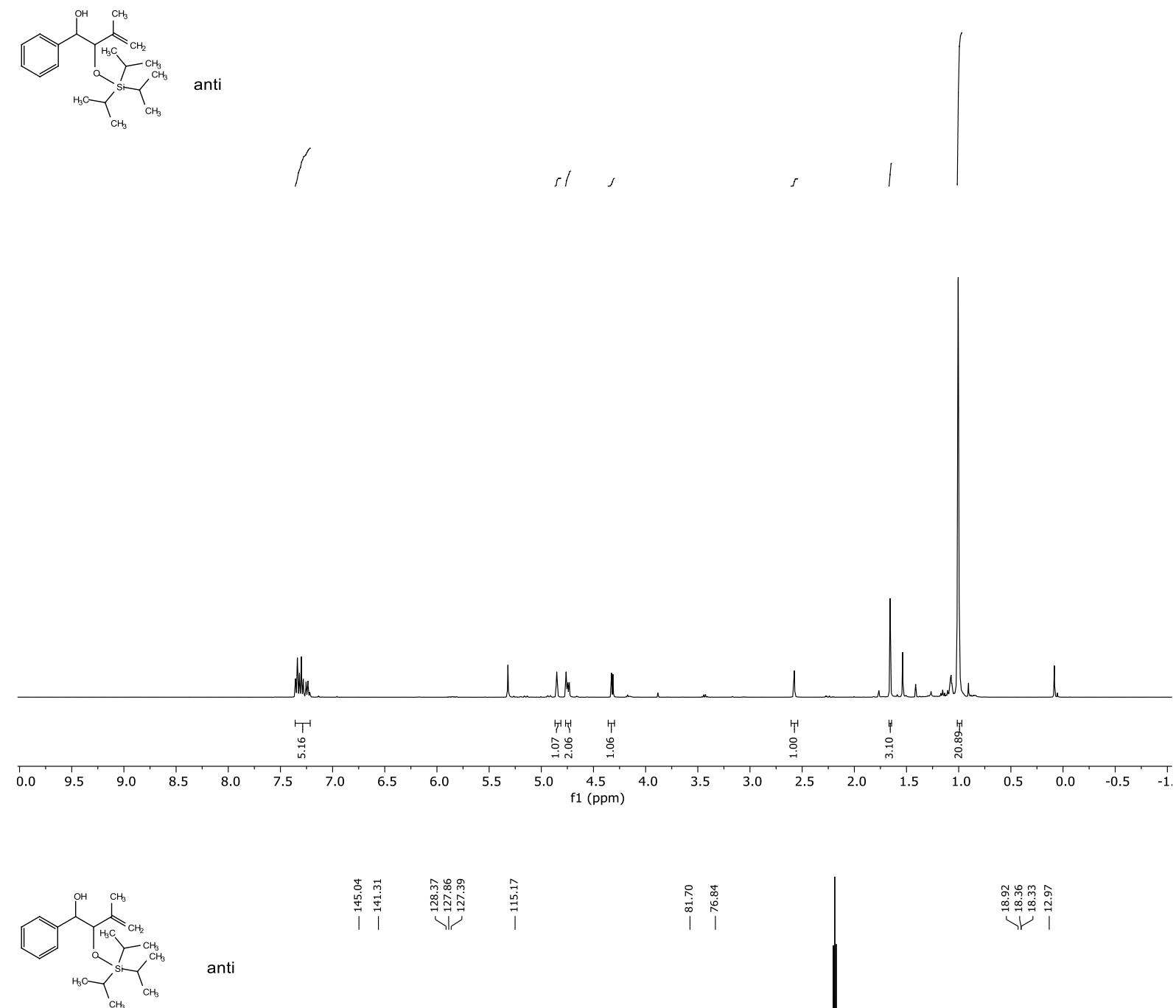

|

采

중ำ

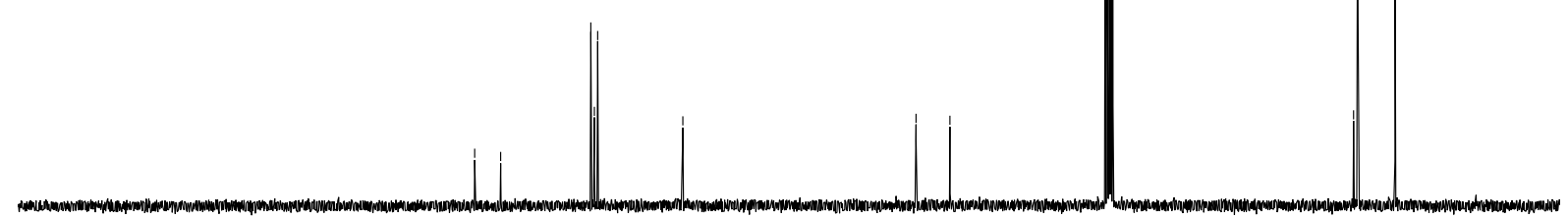

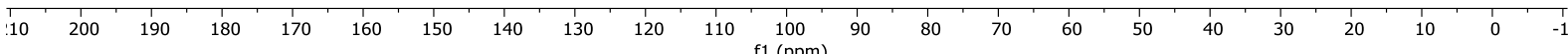



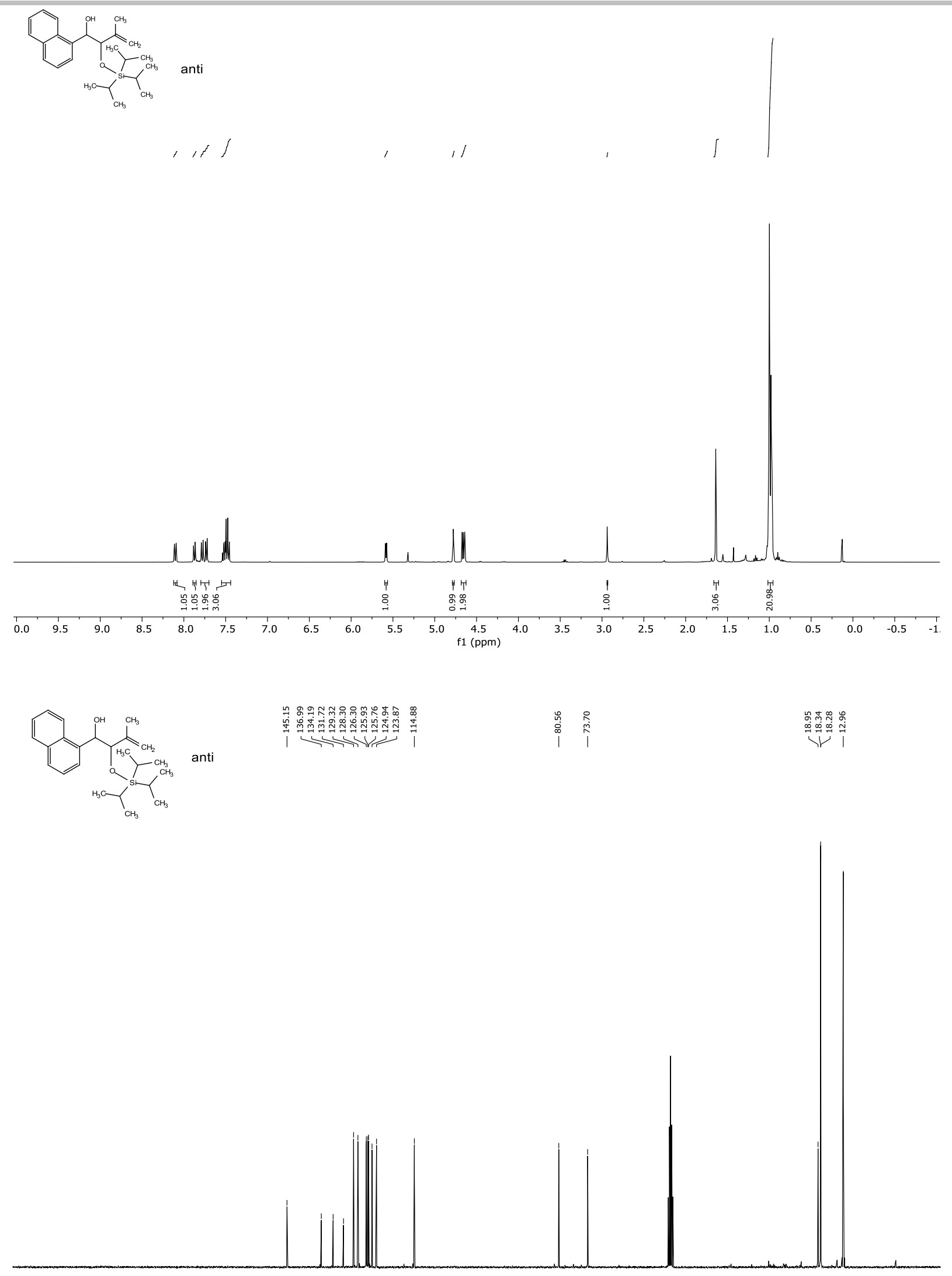

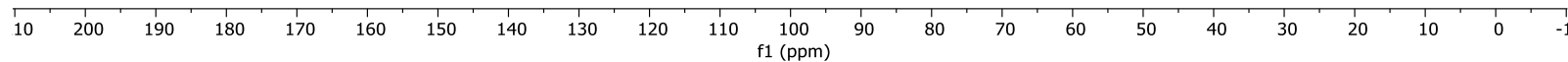


anti

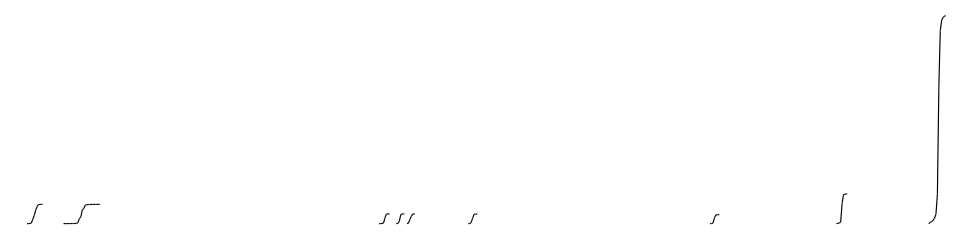

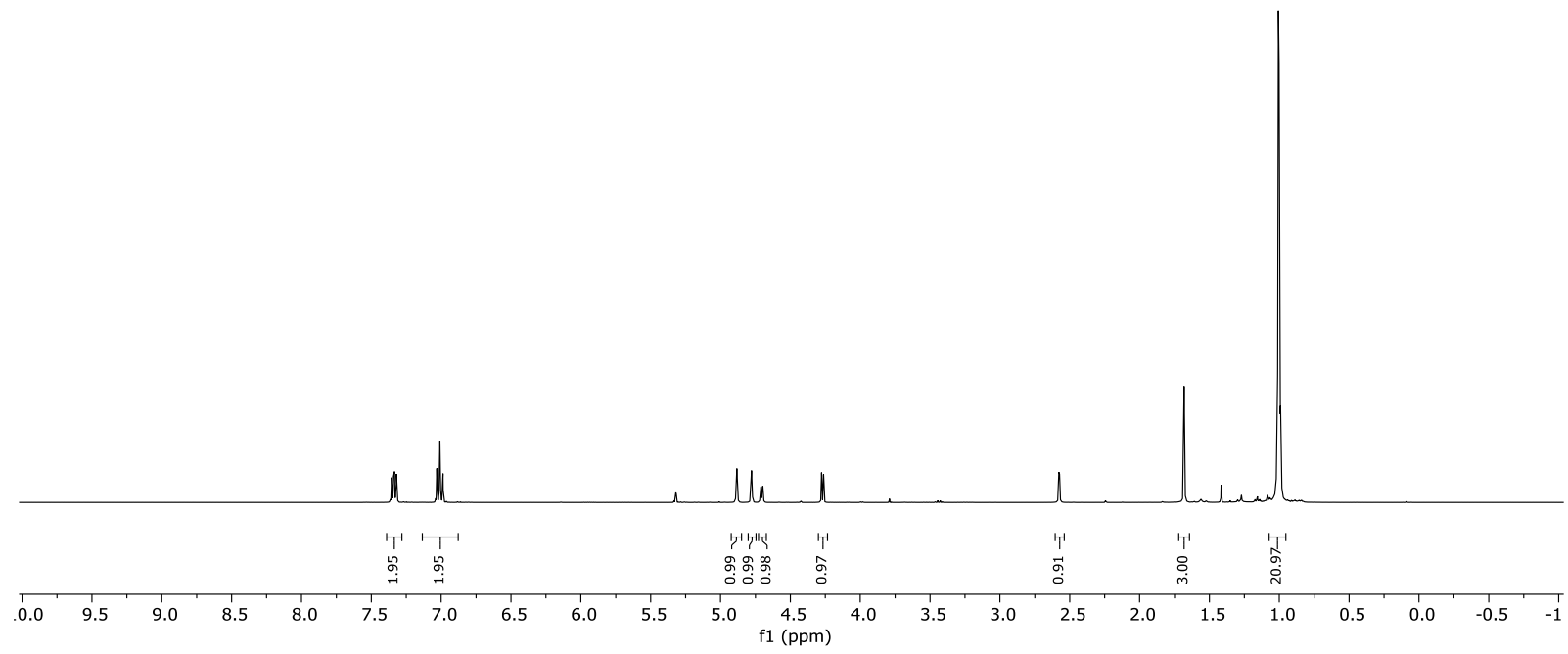

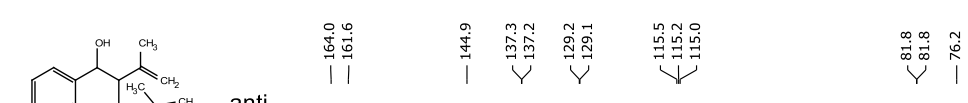

$\underbrace{\infty}$

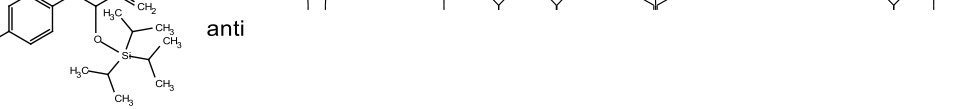

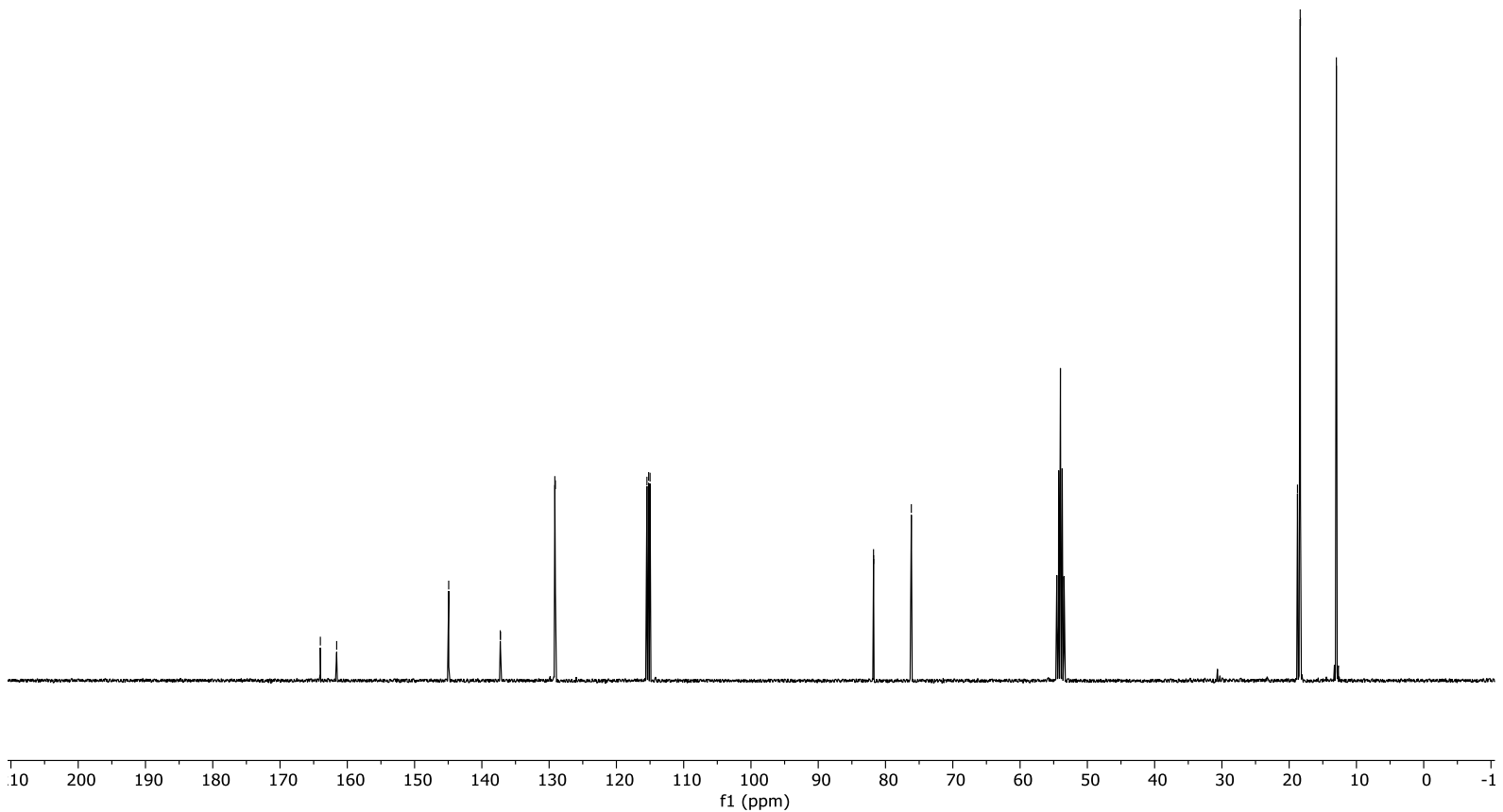




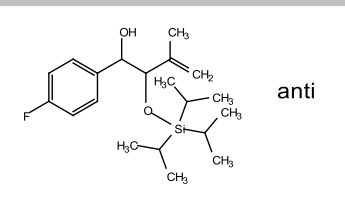

$\stackrel{\overrightarrow{0}}{\overrightarrow{0}}$

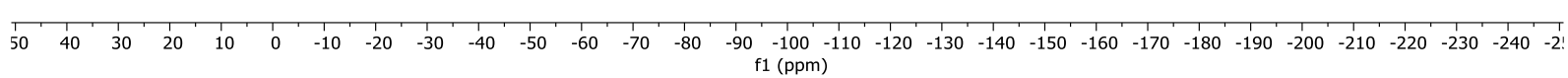
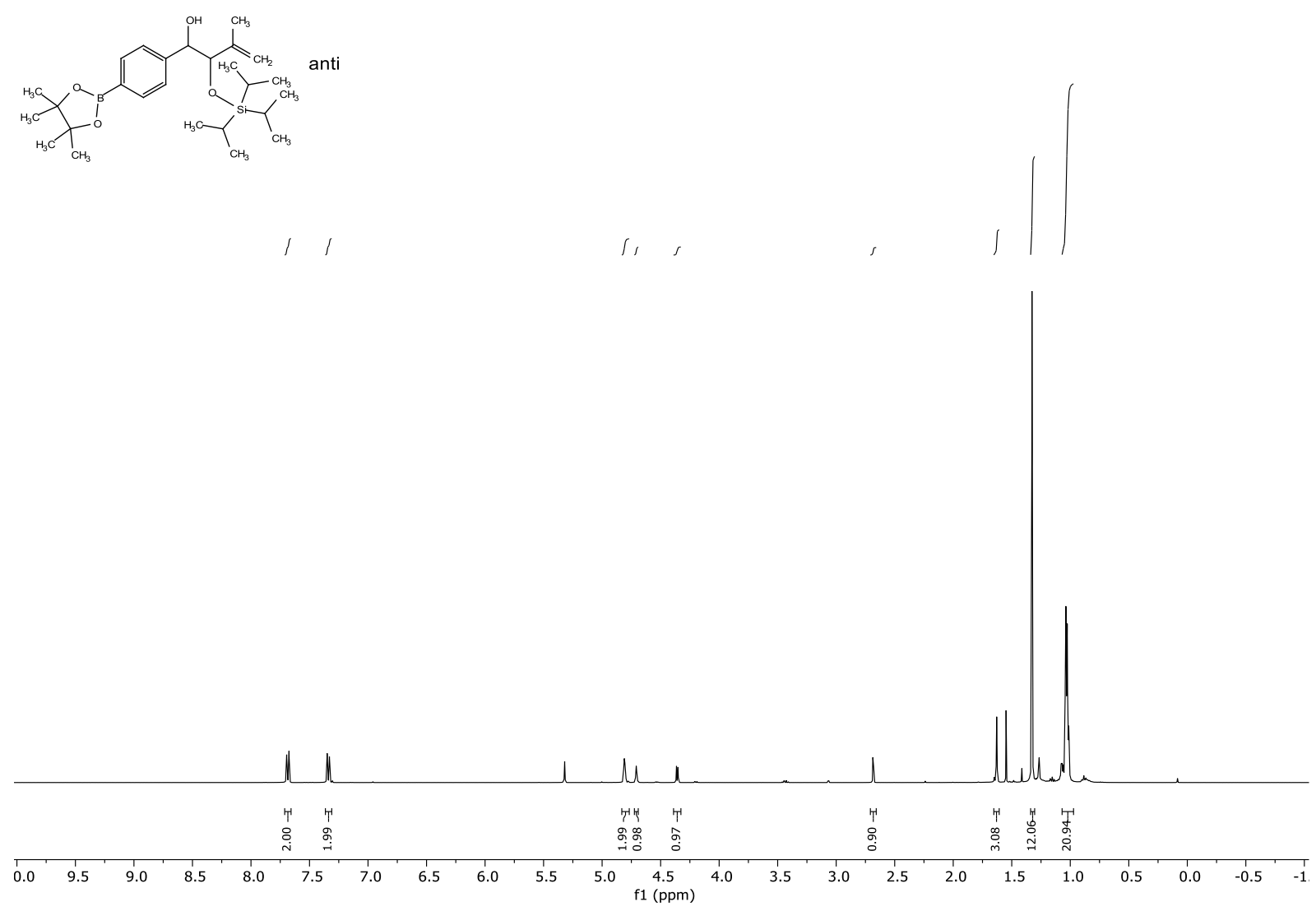

S60 


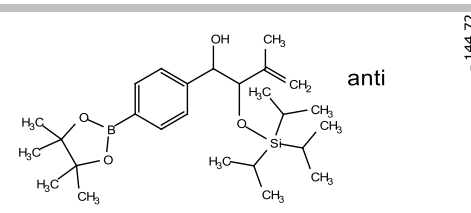

党

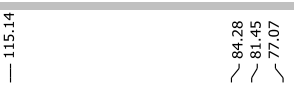

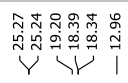
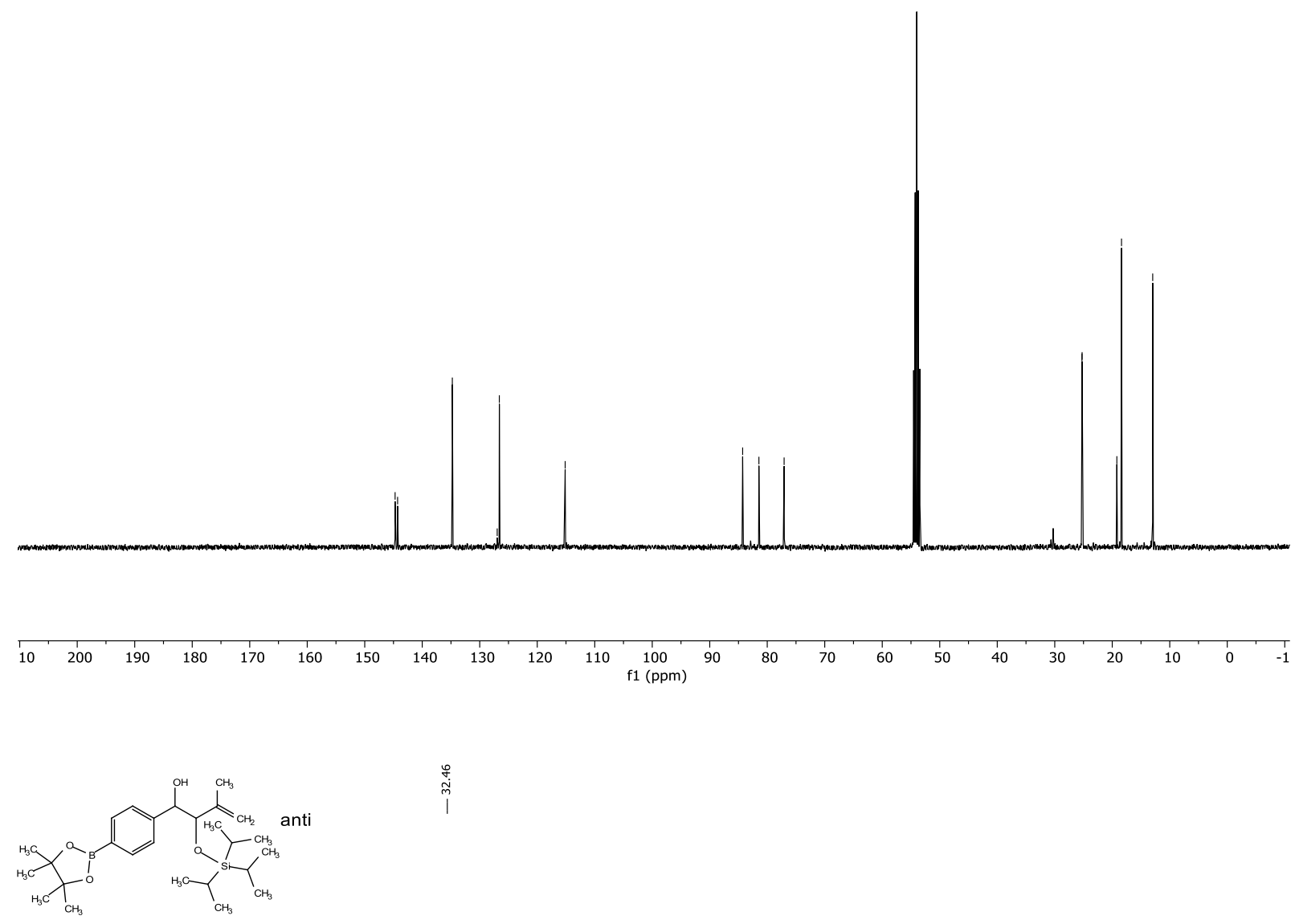

$\underset{\substack{\mathfrak{j} \\ \text { i }}}{1}$
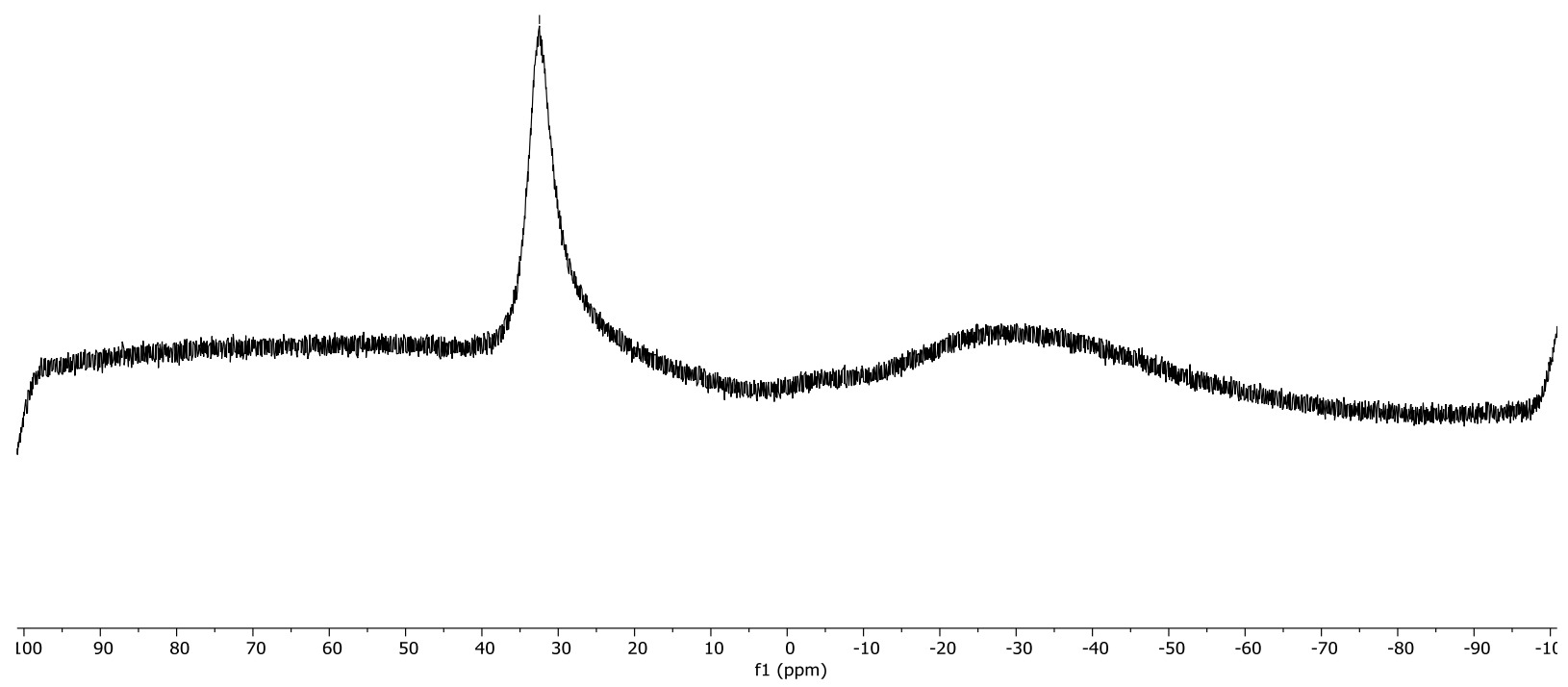

S61 

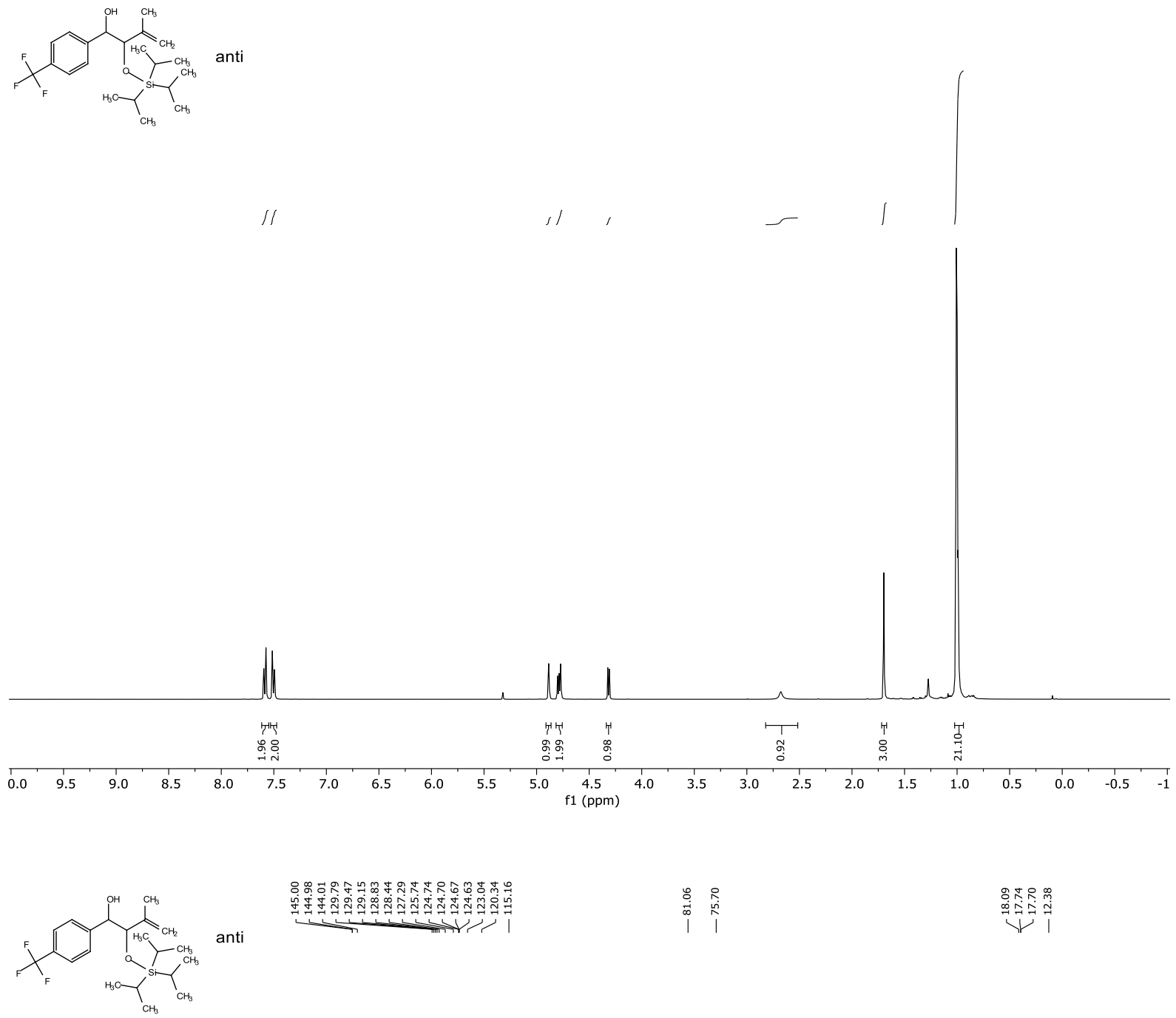

P

象

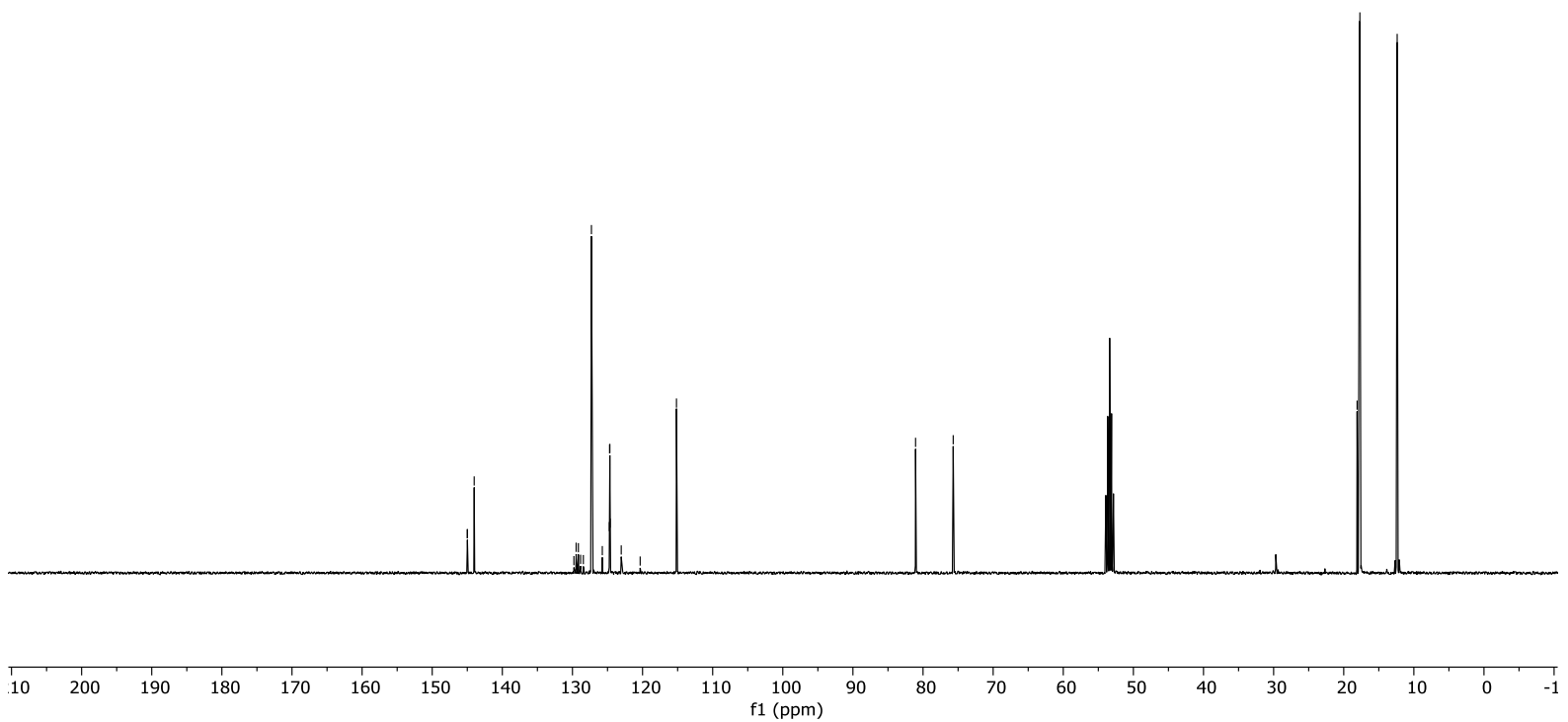



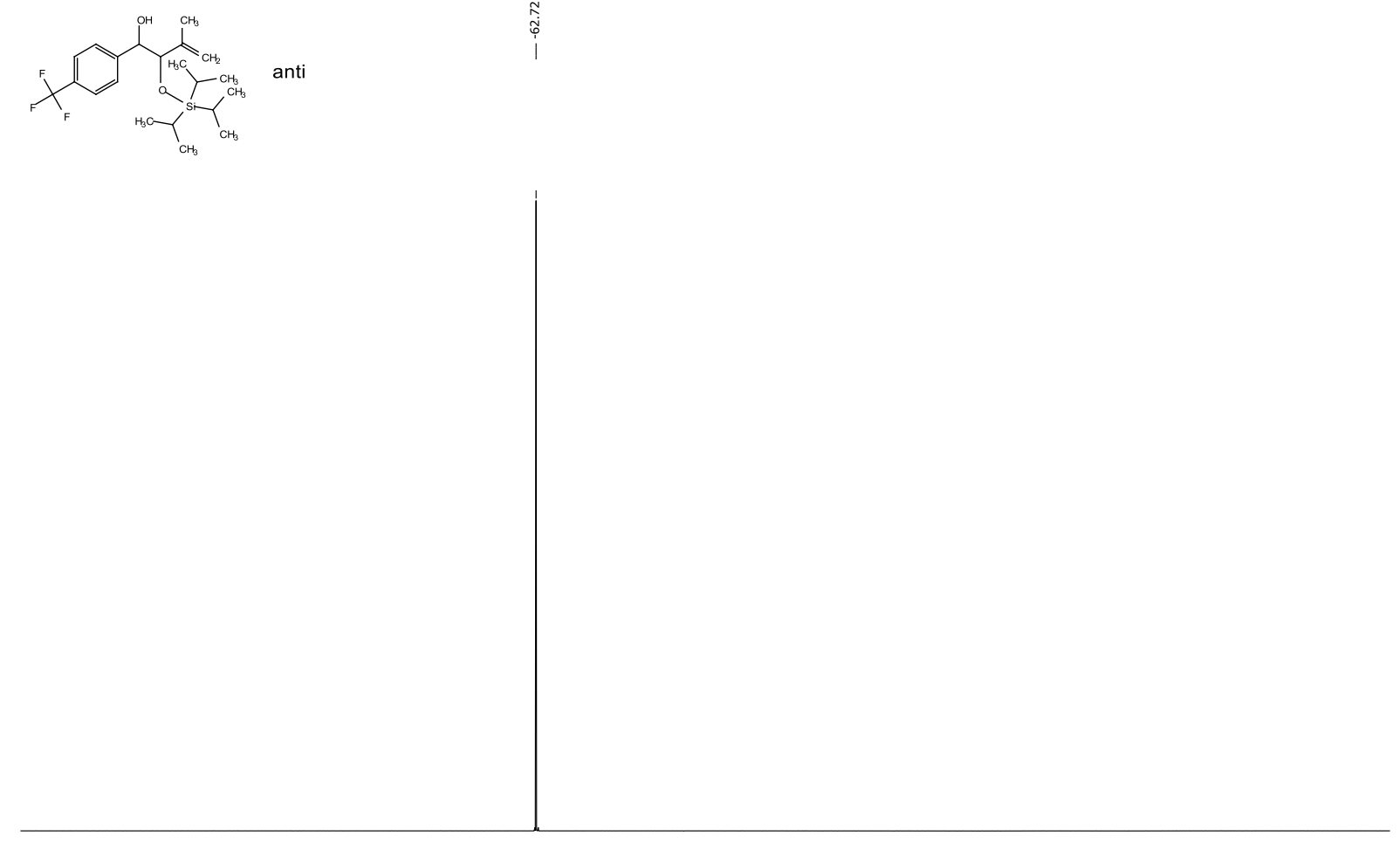

$\begin{array}{lllllllllllllllllllllllllllllllllllll}50 & 40 & 30 & 20 & 10 & 0 & -10 & -20 & -30 & -40 & -50 & -60 & -70 & -80 & -90 & -100 & -110 & -120 & -130 & -140 & -150 & -160 & -170 & -180 & -190 & -200 & -210 & -220 & -230 & -240 & -2 !\end{array}$

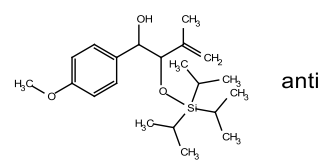

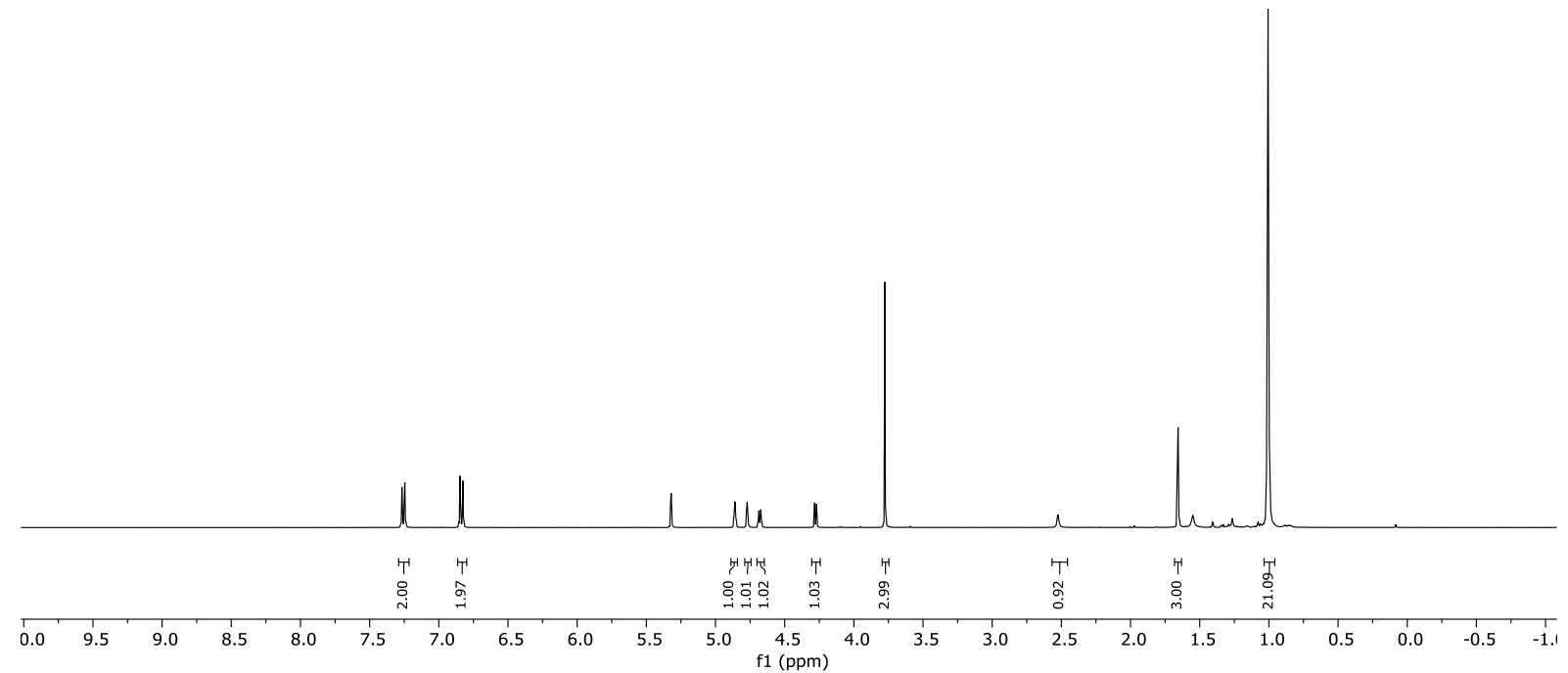




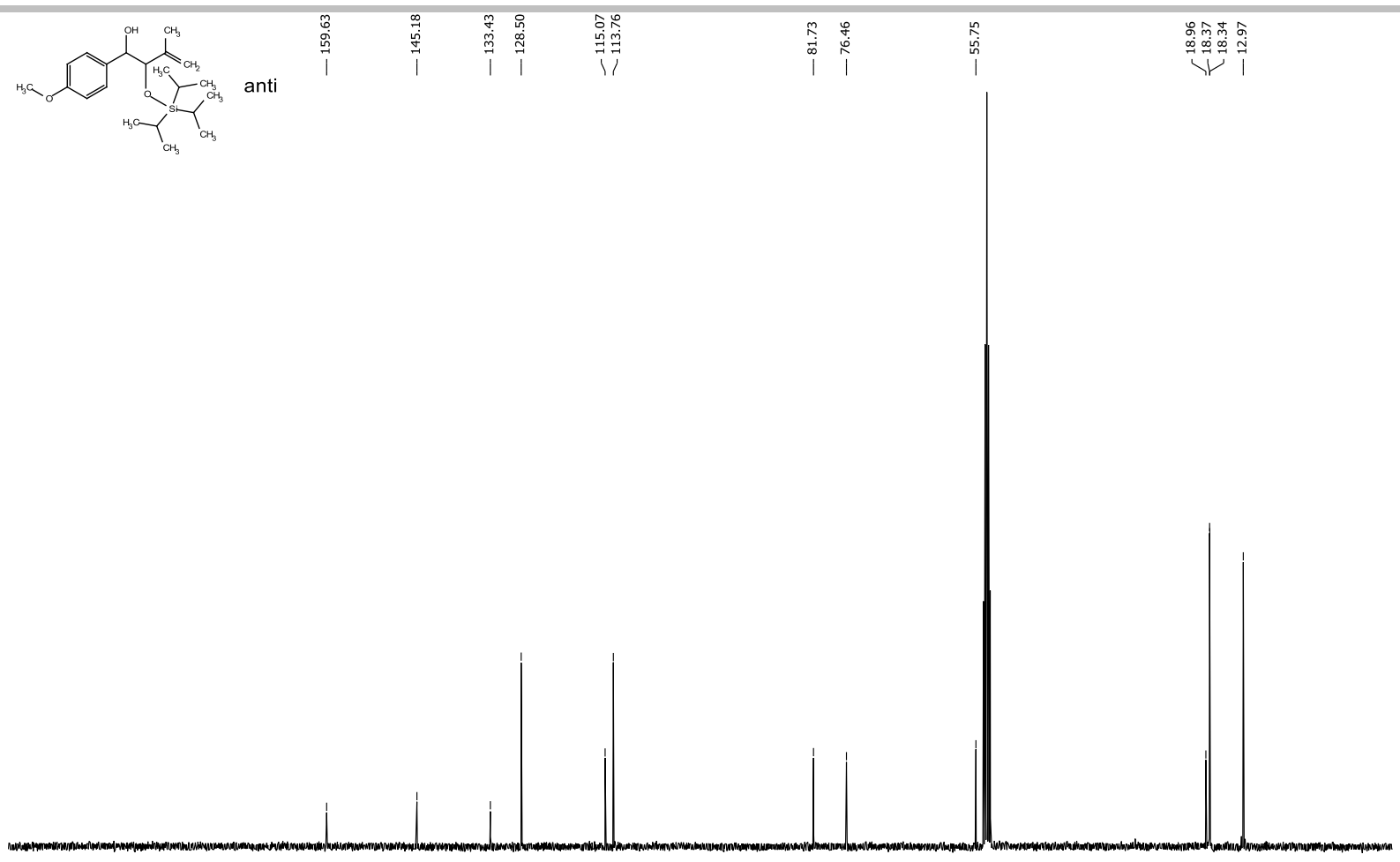

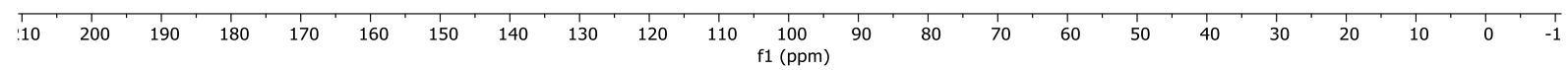
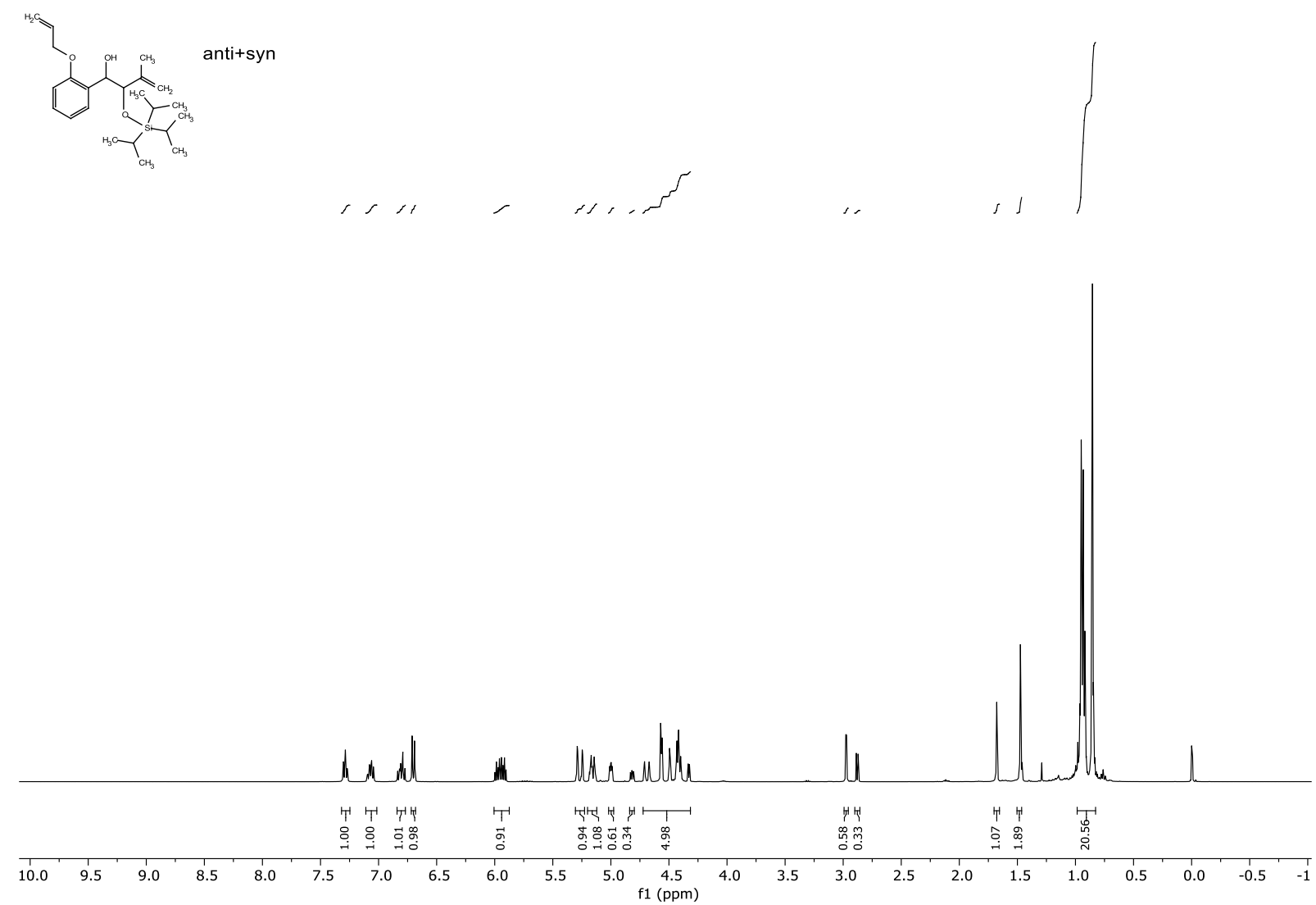

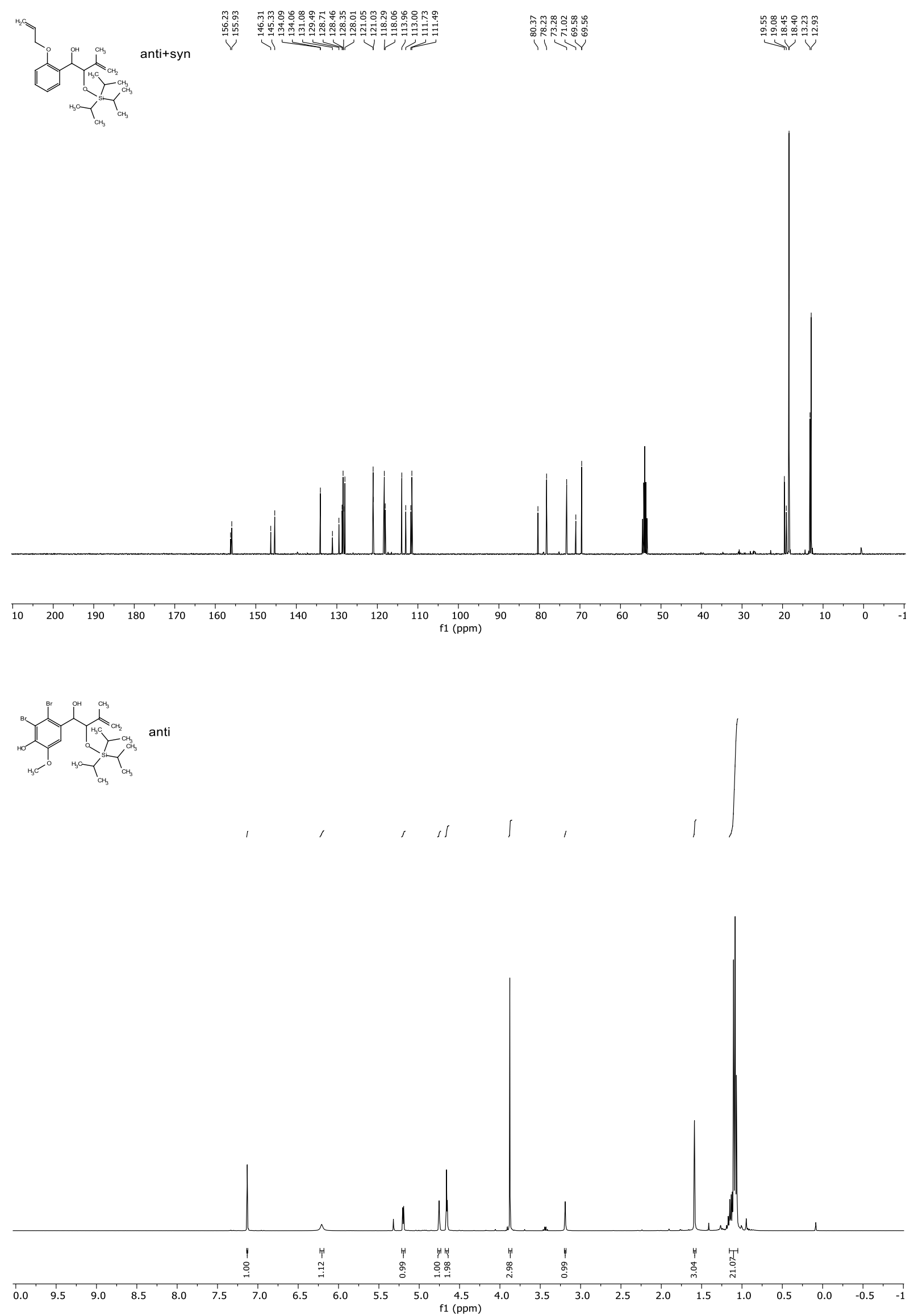


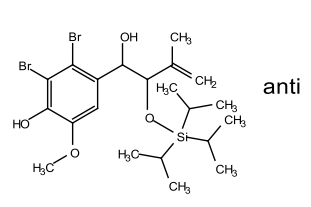

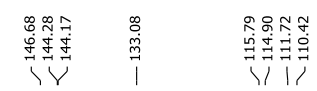

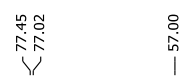

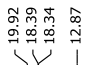

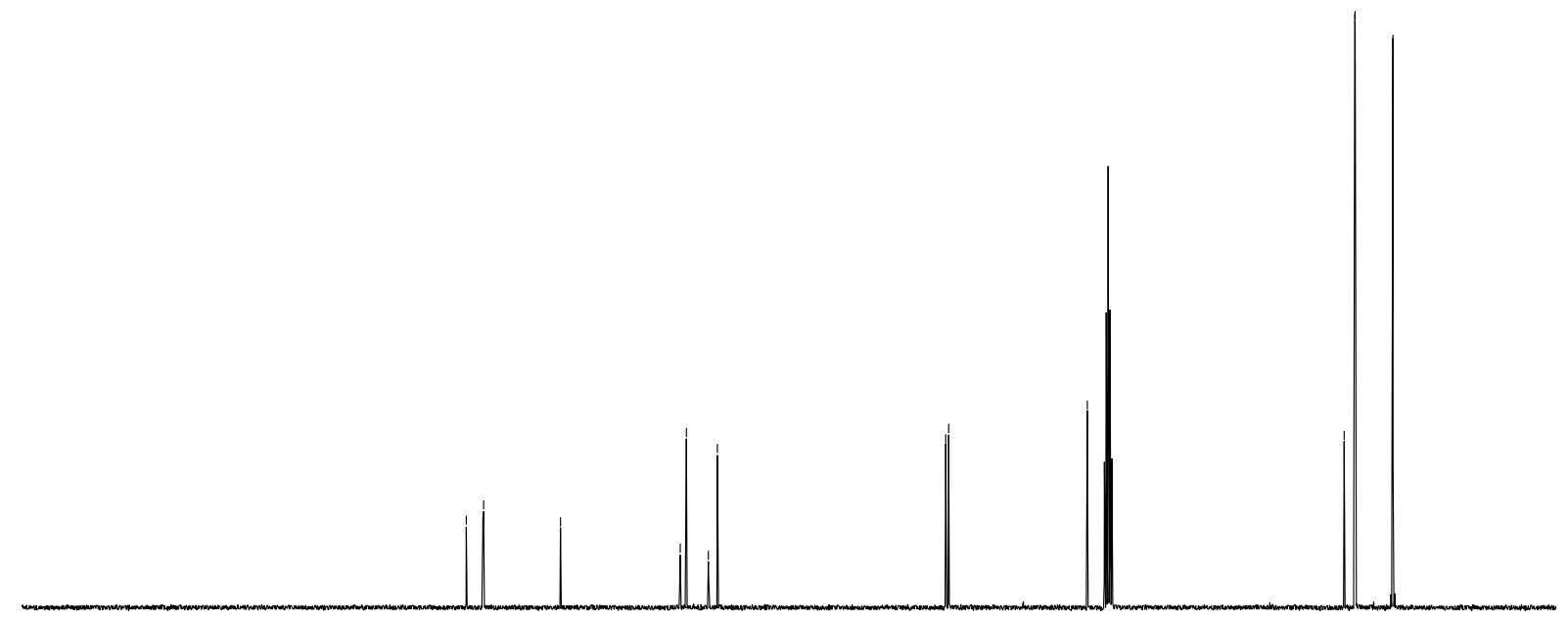

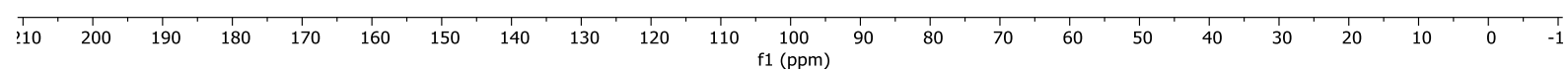
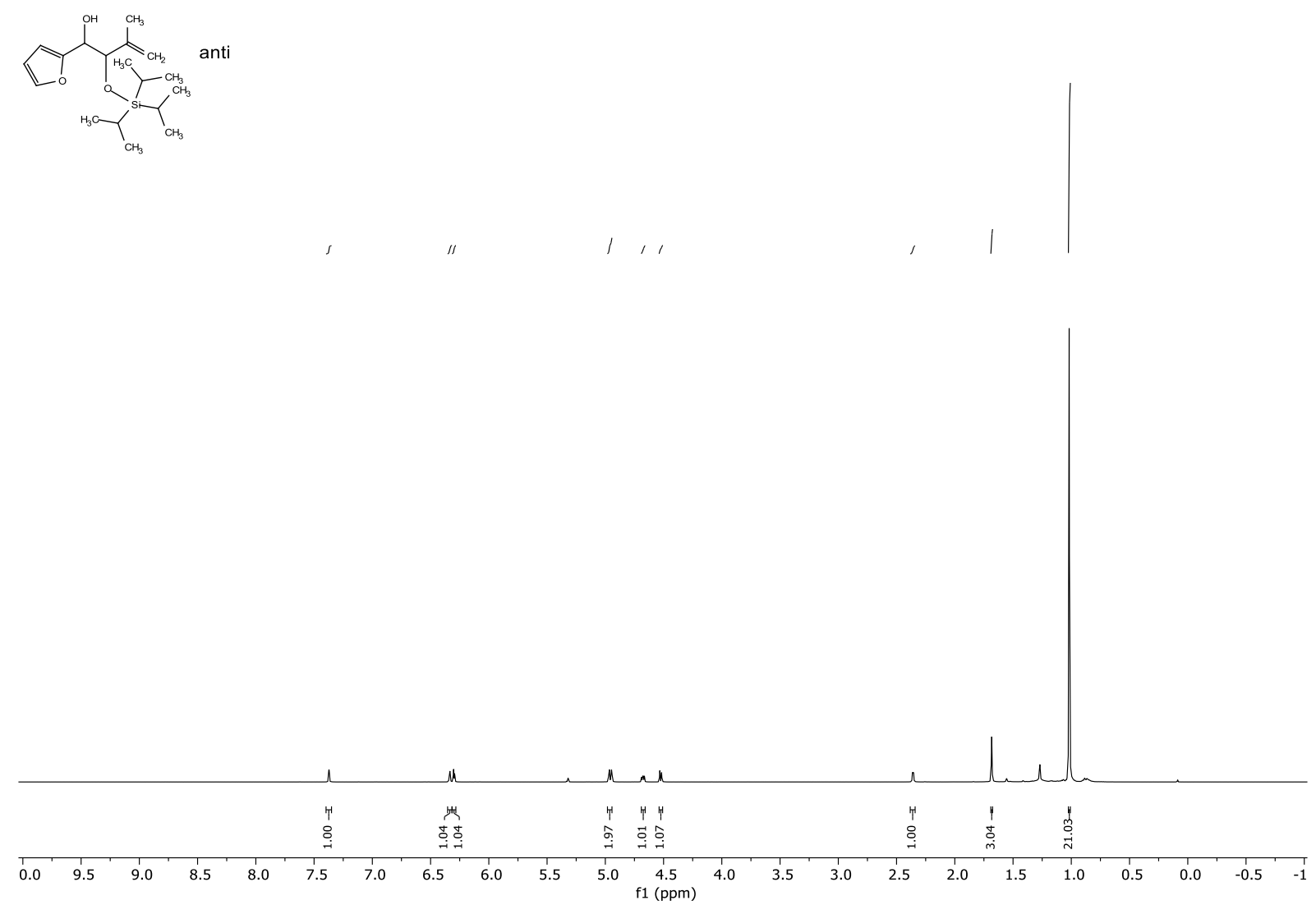

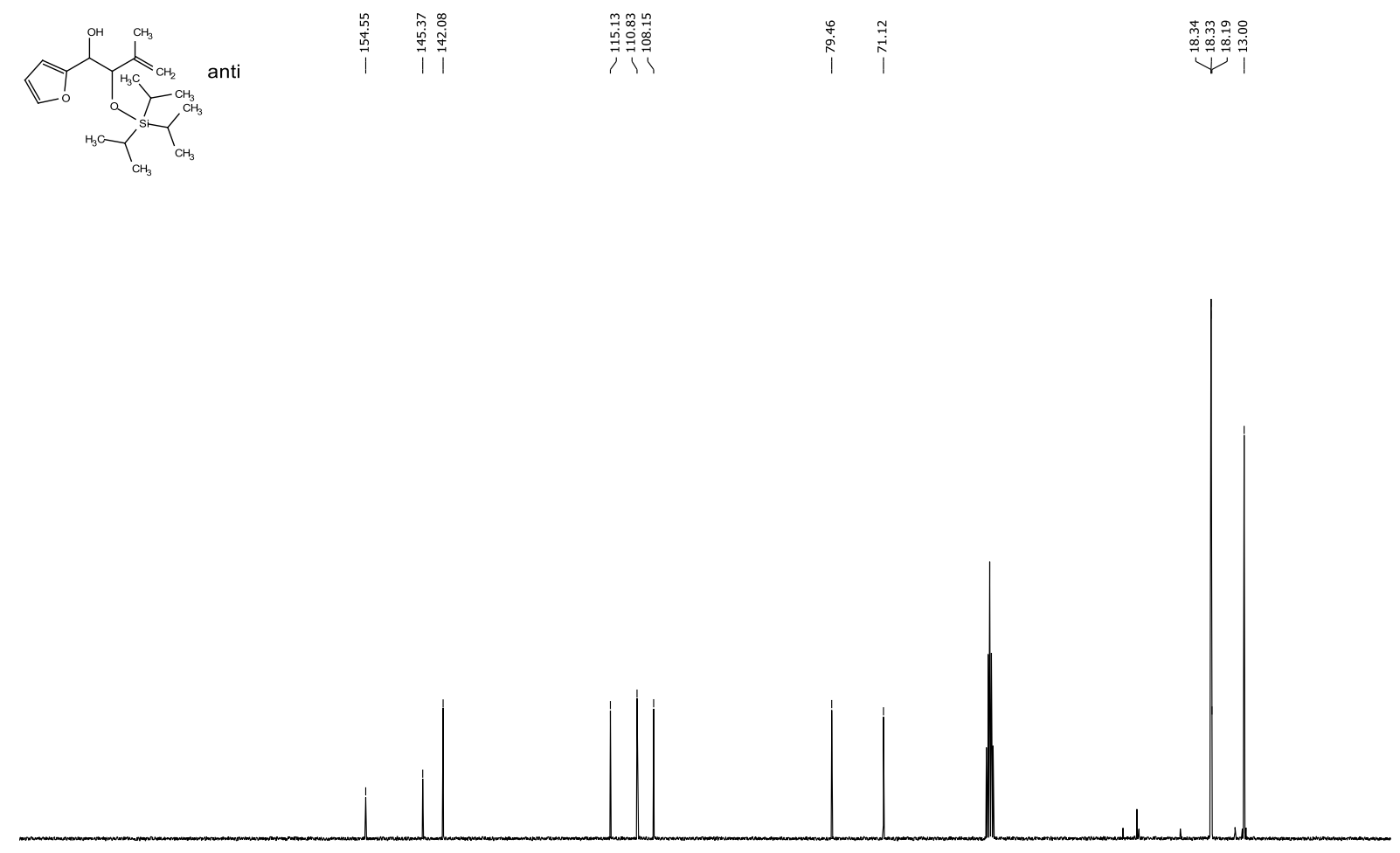

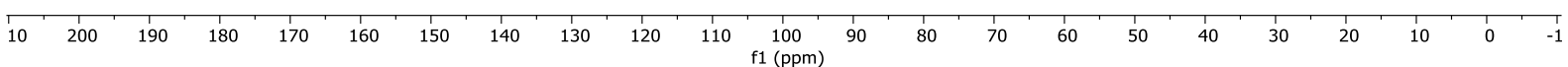
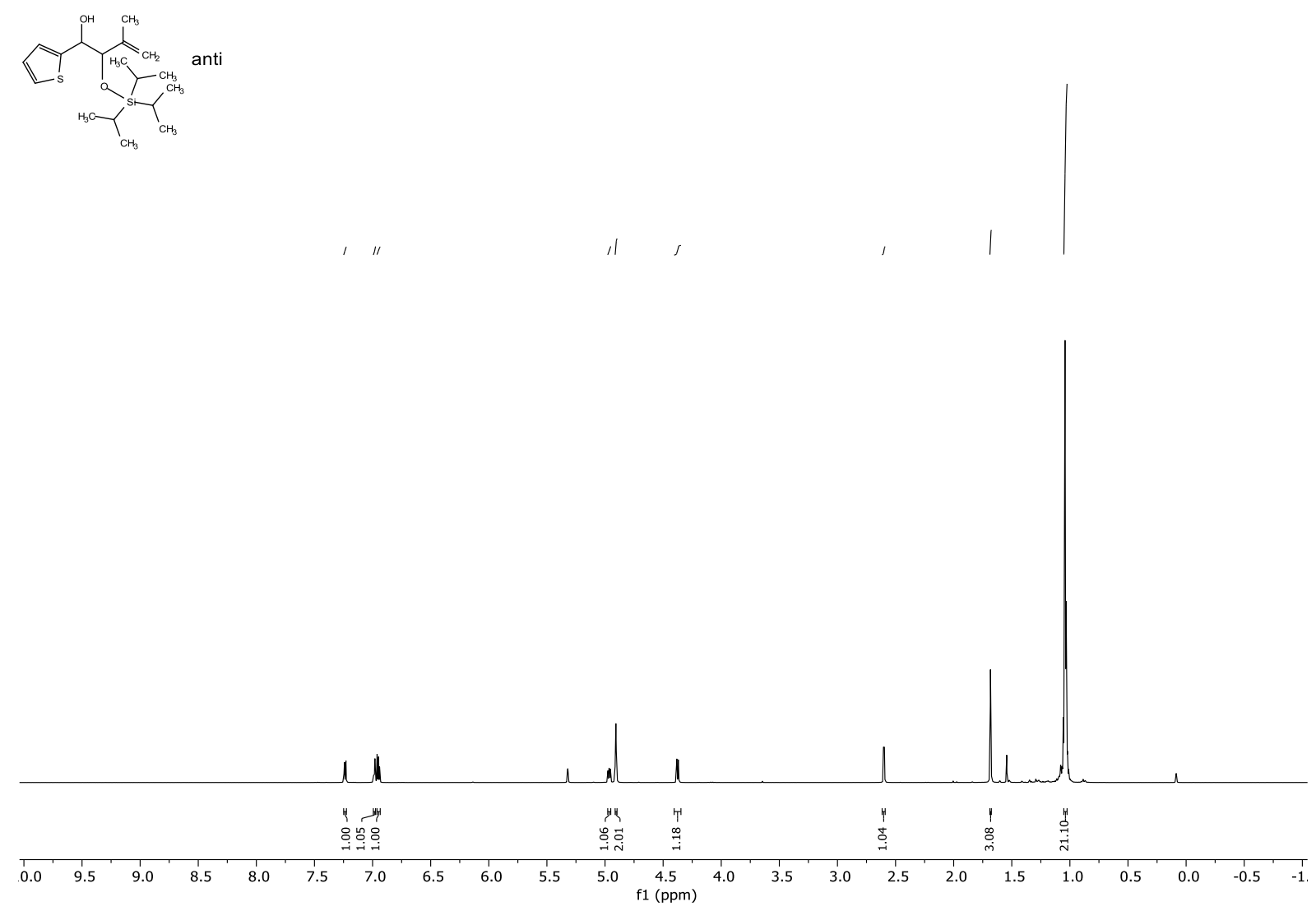


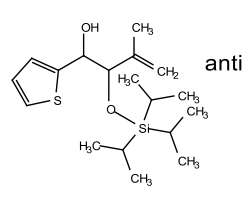

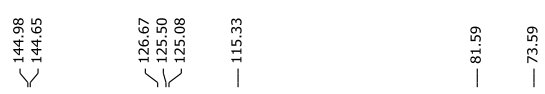

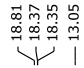

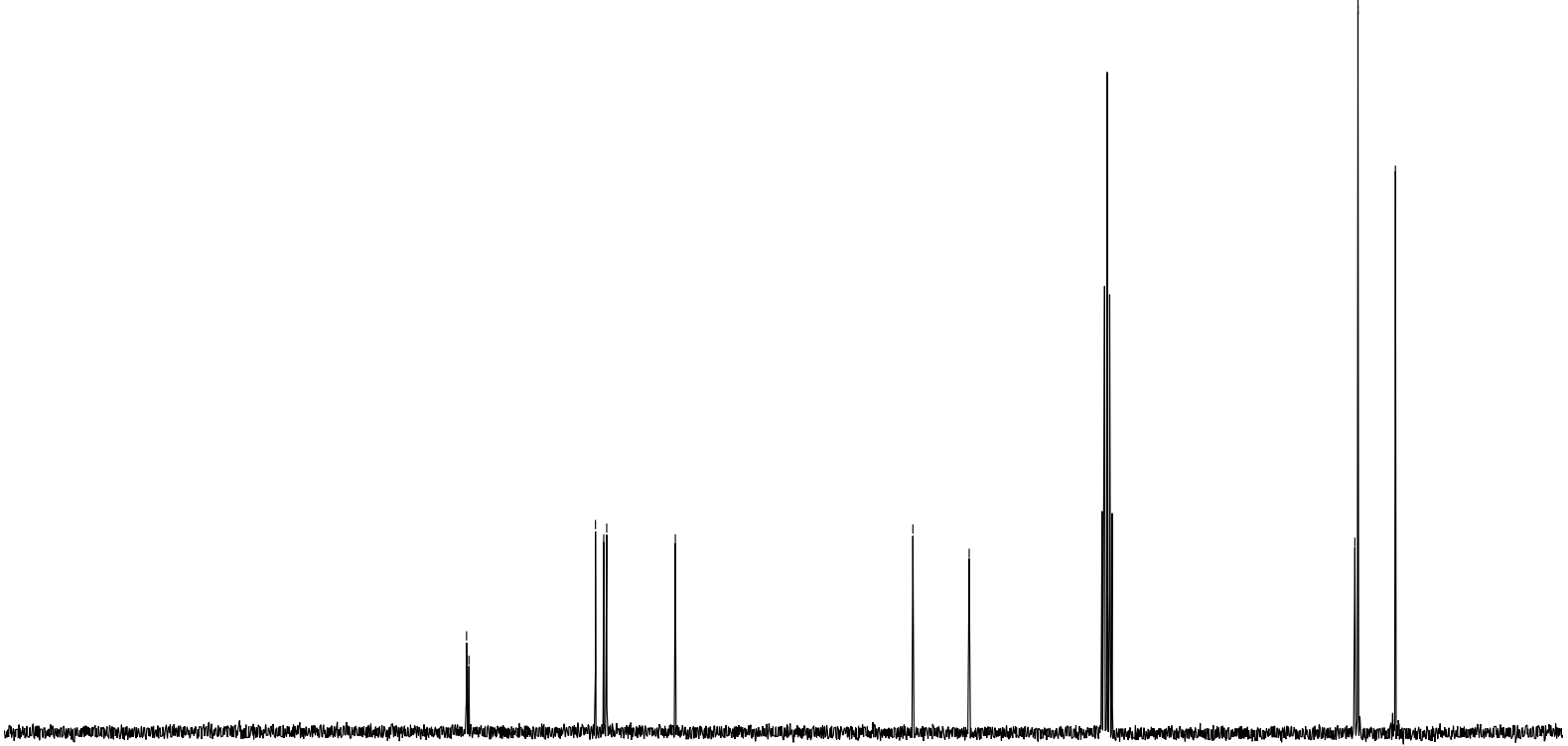

$\begin{array}{llllllllllllllllllllllll}\mid & 1 \\ 10 & 200 & 190 & 180 & 170 & 160 & 150 & 140 & 130 & 120 & 110 & \begin{array}{c}100 \\ \mathrm{f} 1(\mathrm{ppm})\end{array} & 90 & 80 & 70 & 60 & 50 & 40 & 30 & 20 & 10 & 0 & -1\end{array}$

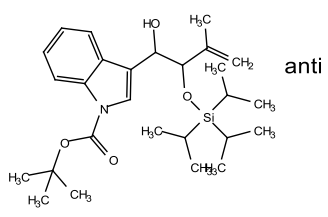

1
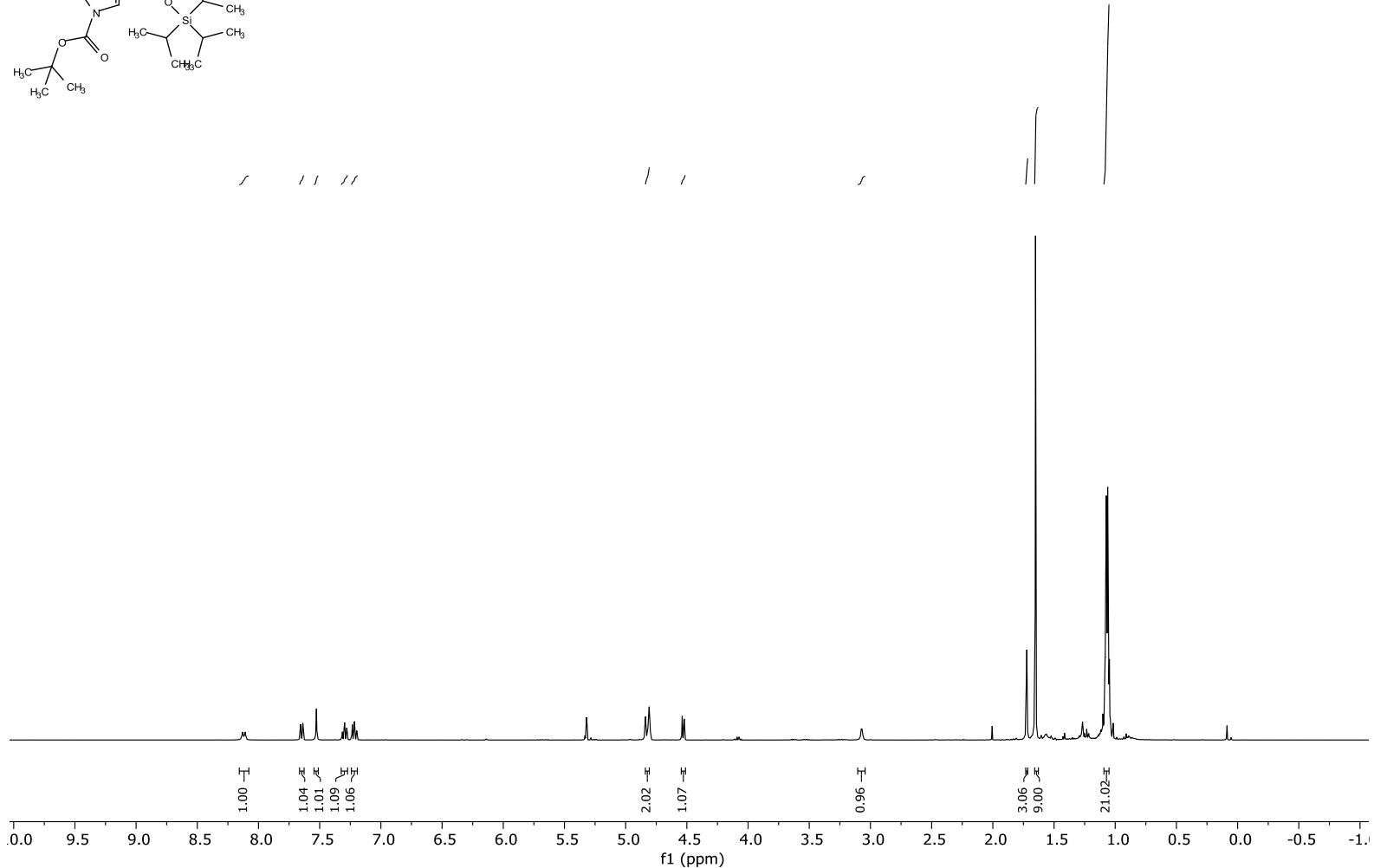

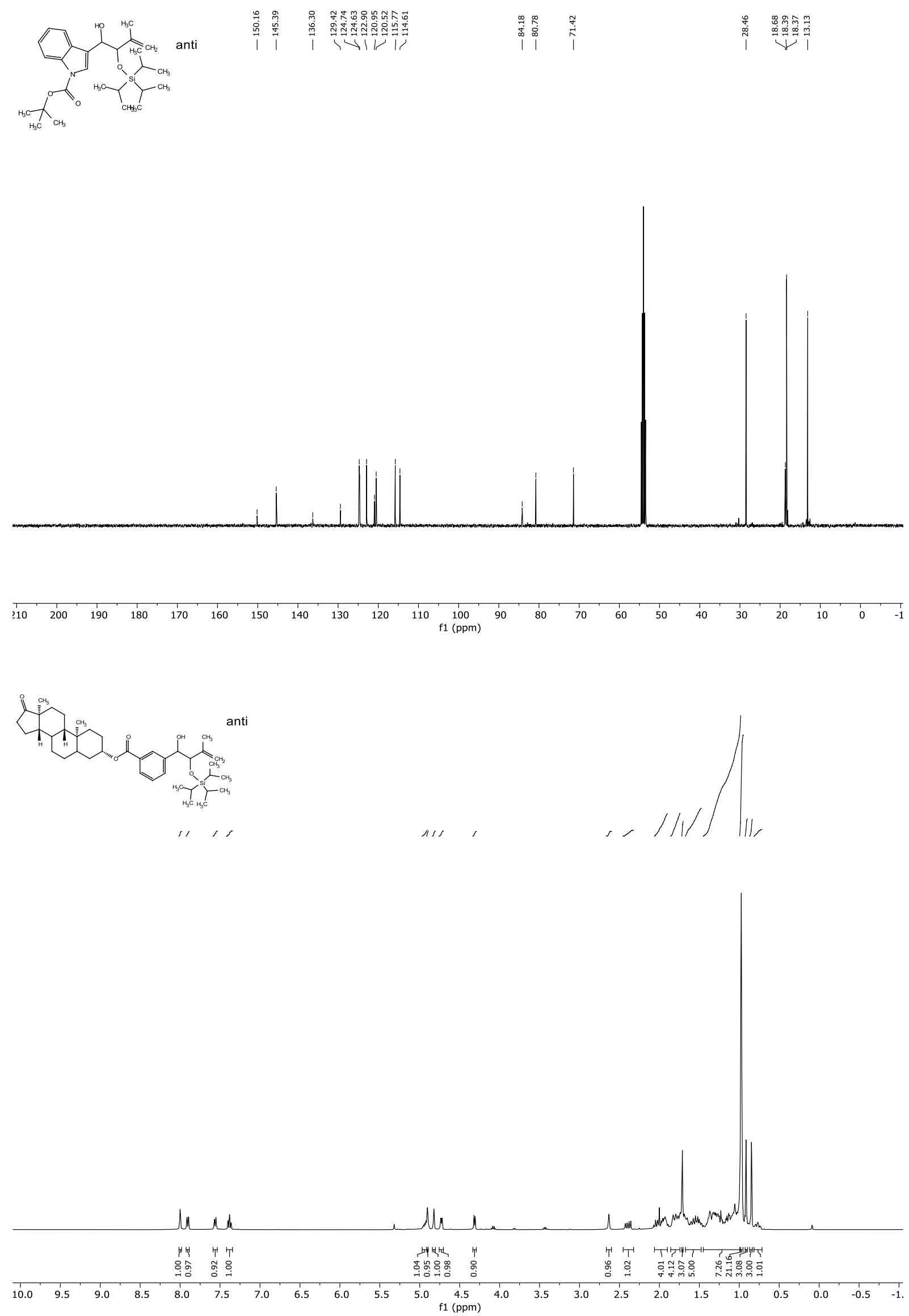


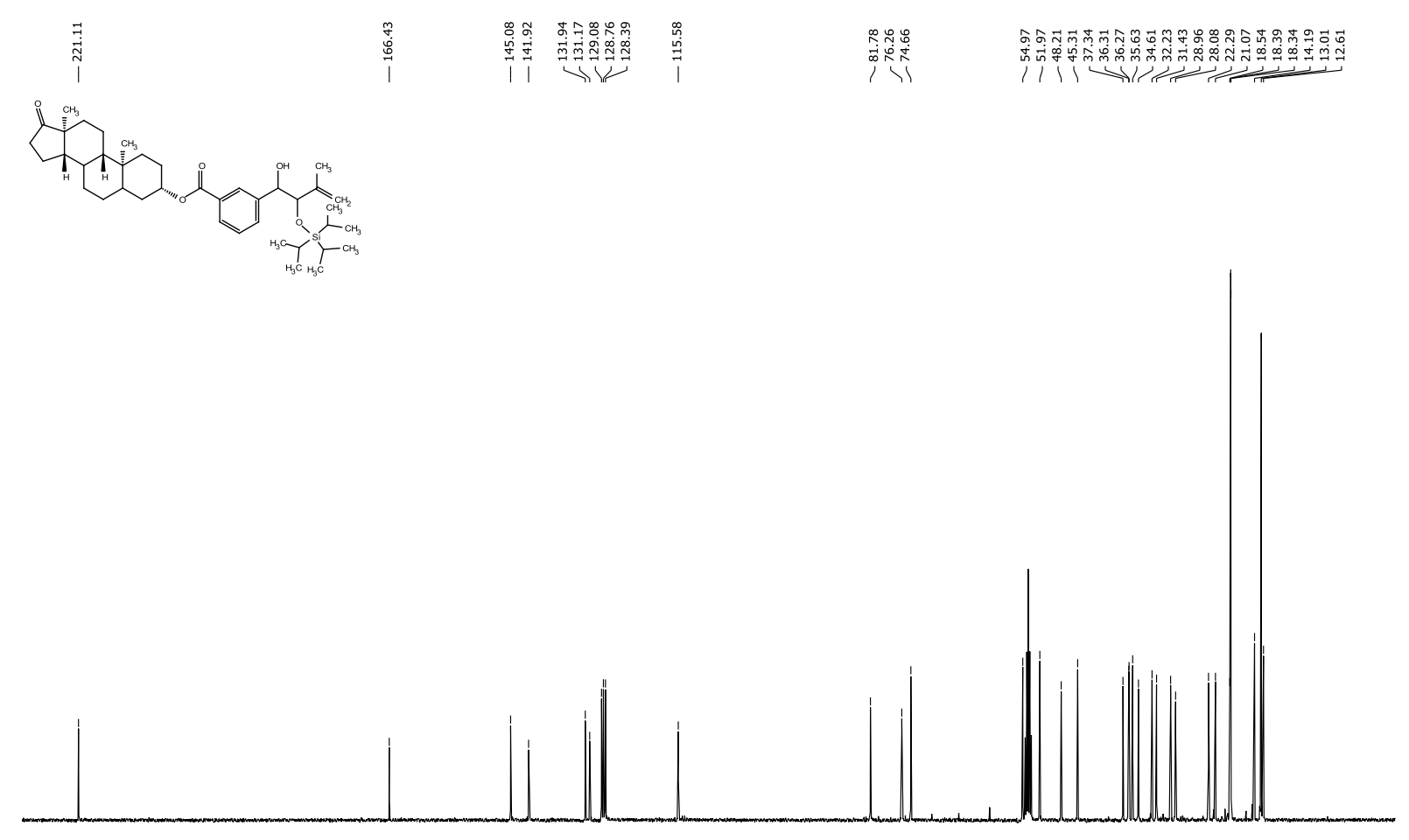

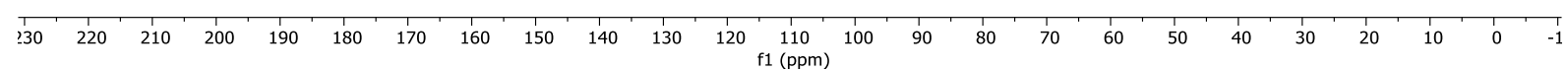<smiles>C=C(C)C(OS(C(C)C)(C(C)C)C(C)C)C(O)C1CCCCC1</smiles>

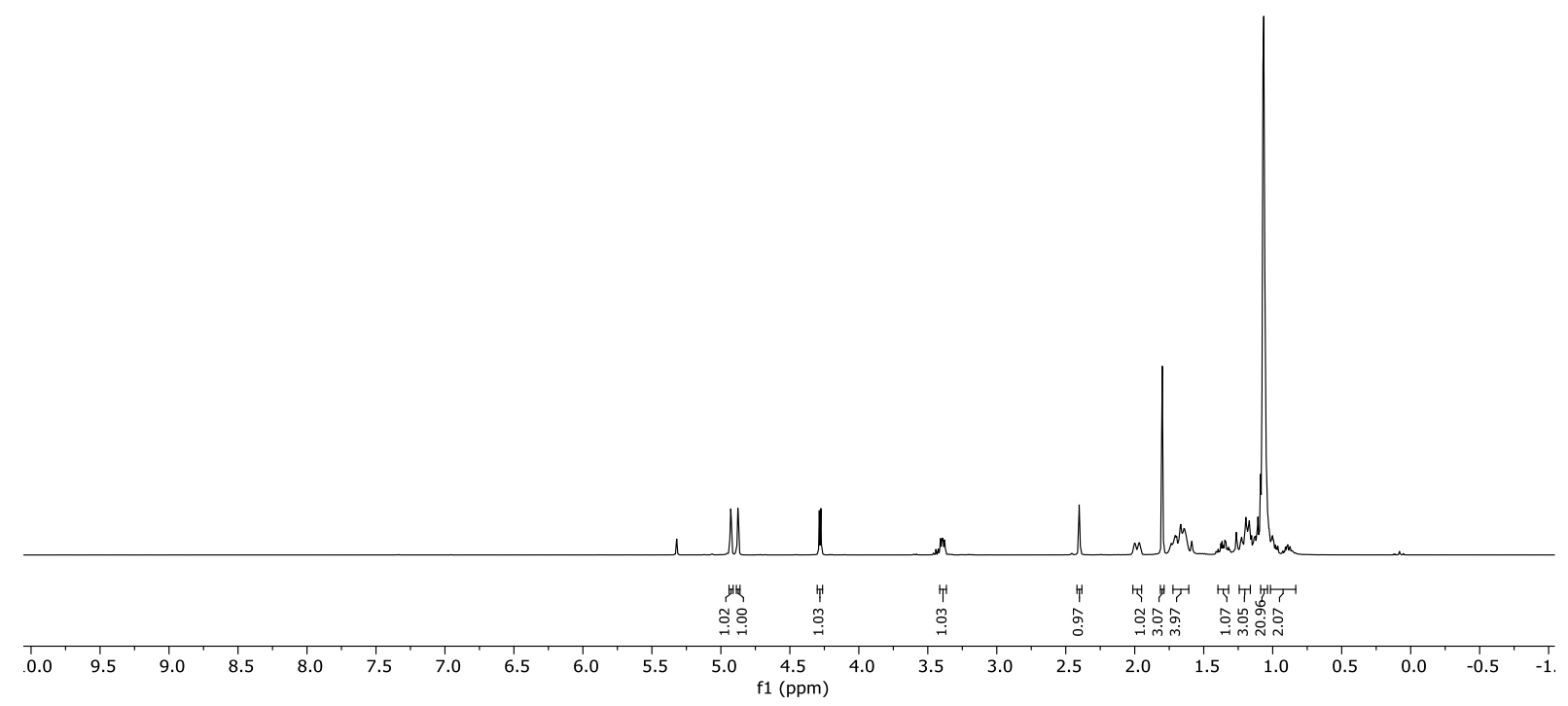




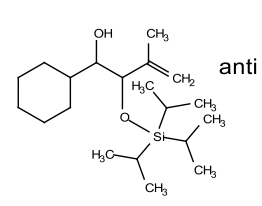

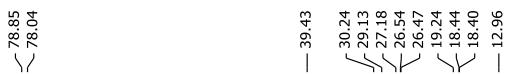
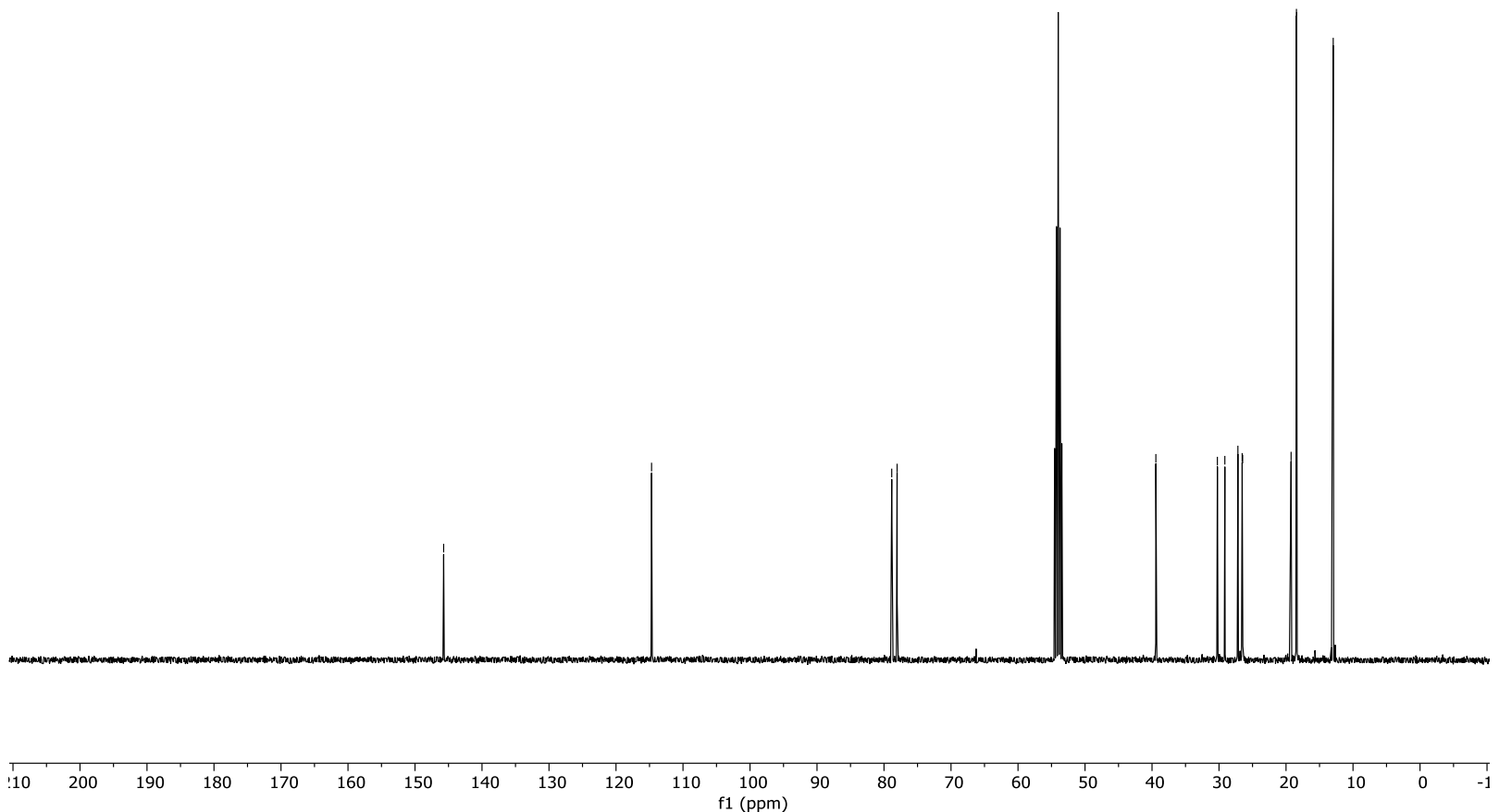

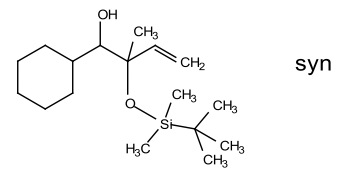

Wil. H H III
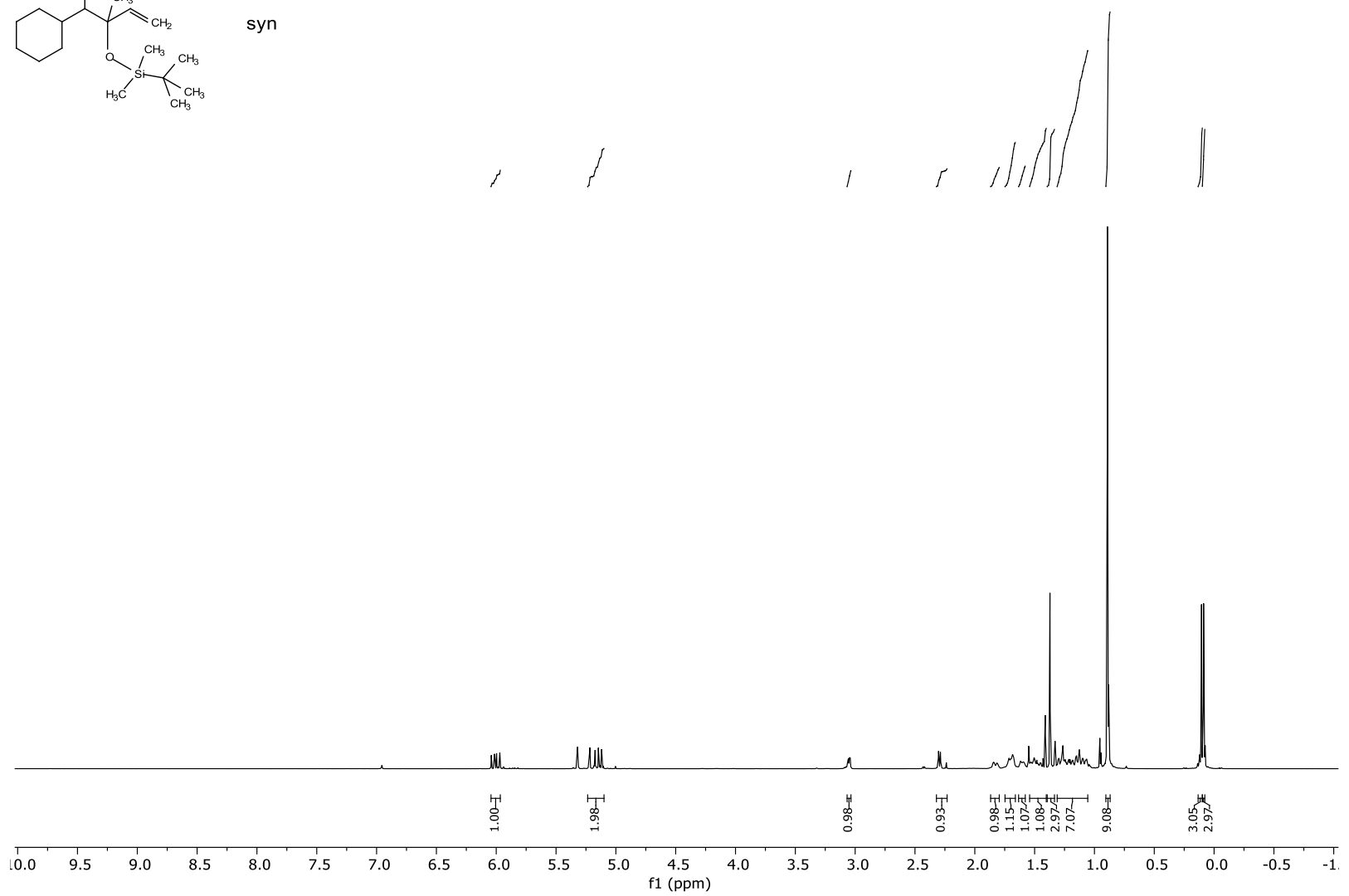


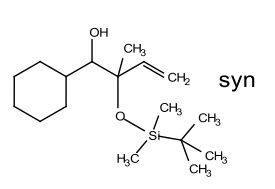

$\stackrel{\overrightarrow{7}}{\underline{p}}$

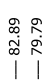

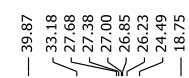

ve
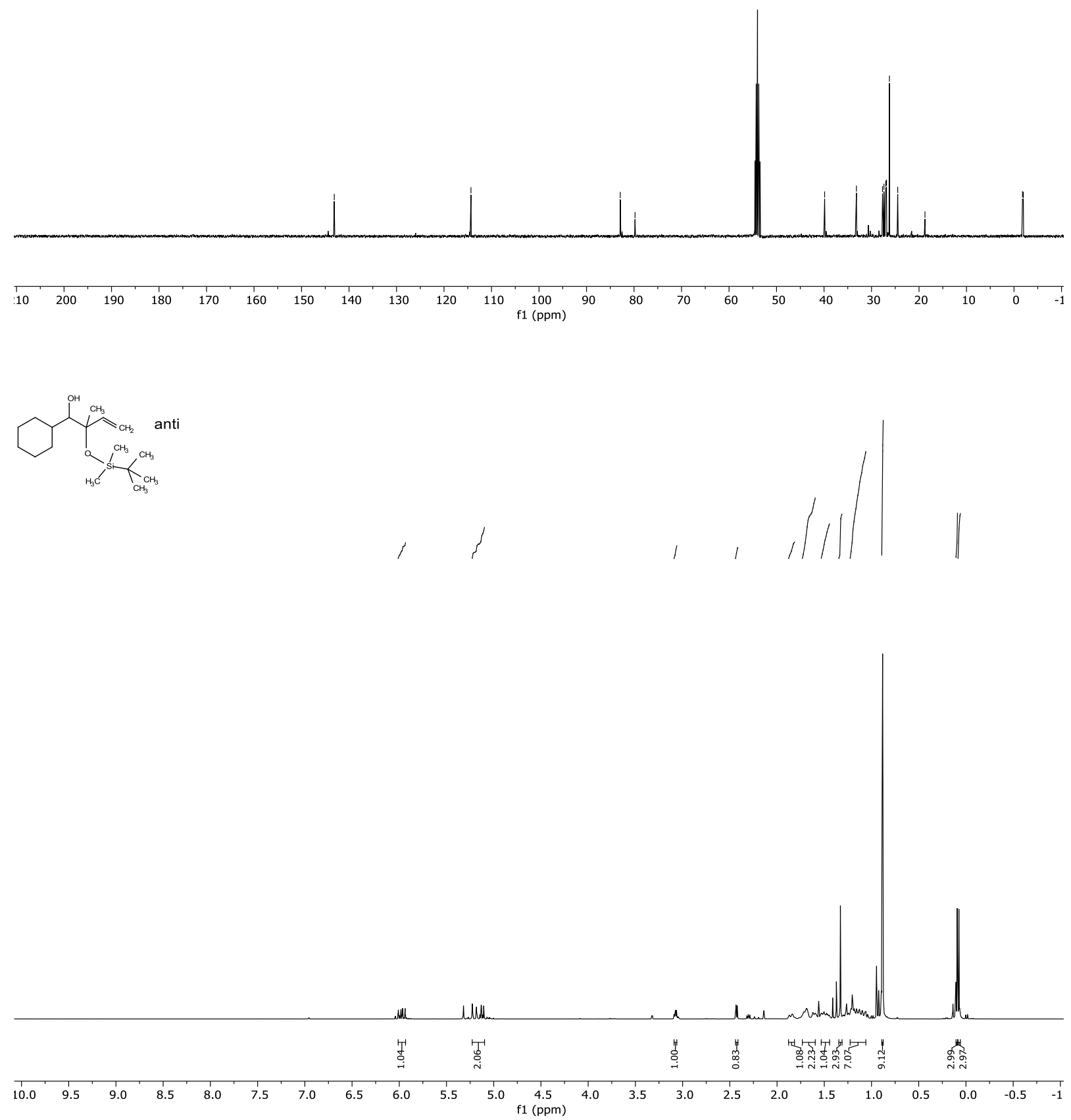

S72 

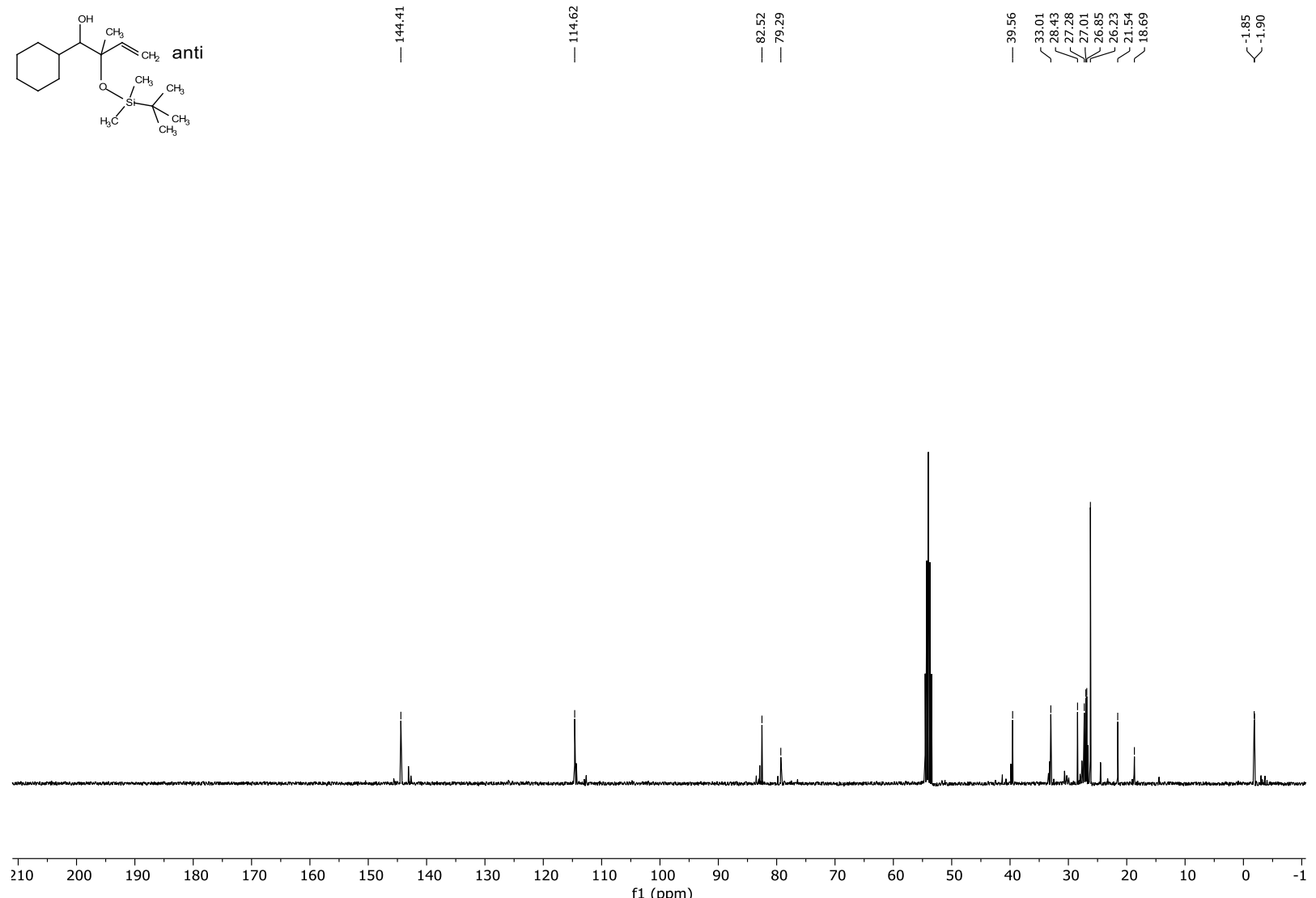<smiles>C=CC(O[PH](C(C)C)(C(C)C)C(C)C)C(O)C1CCCCC1</smiles>

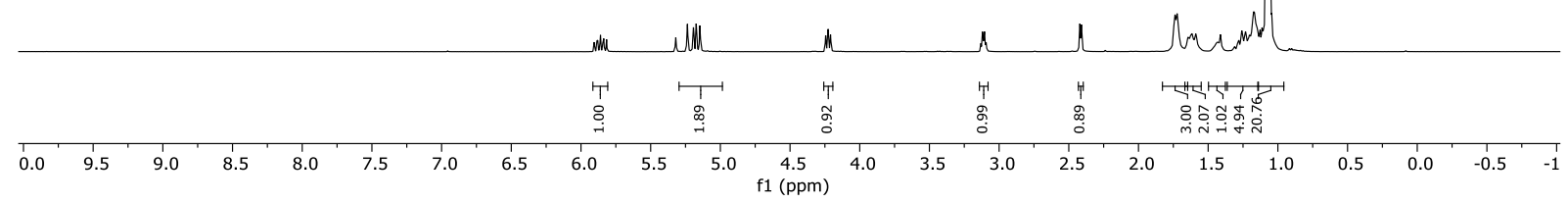



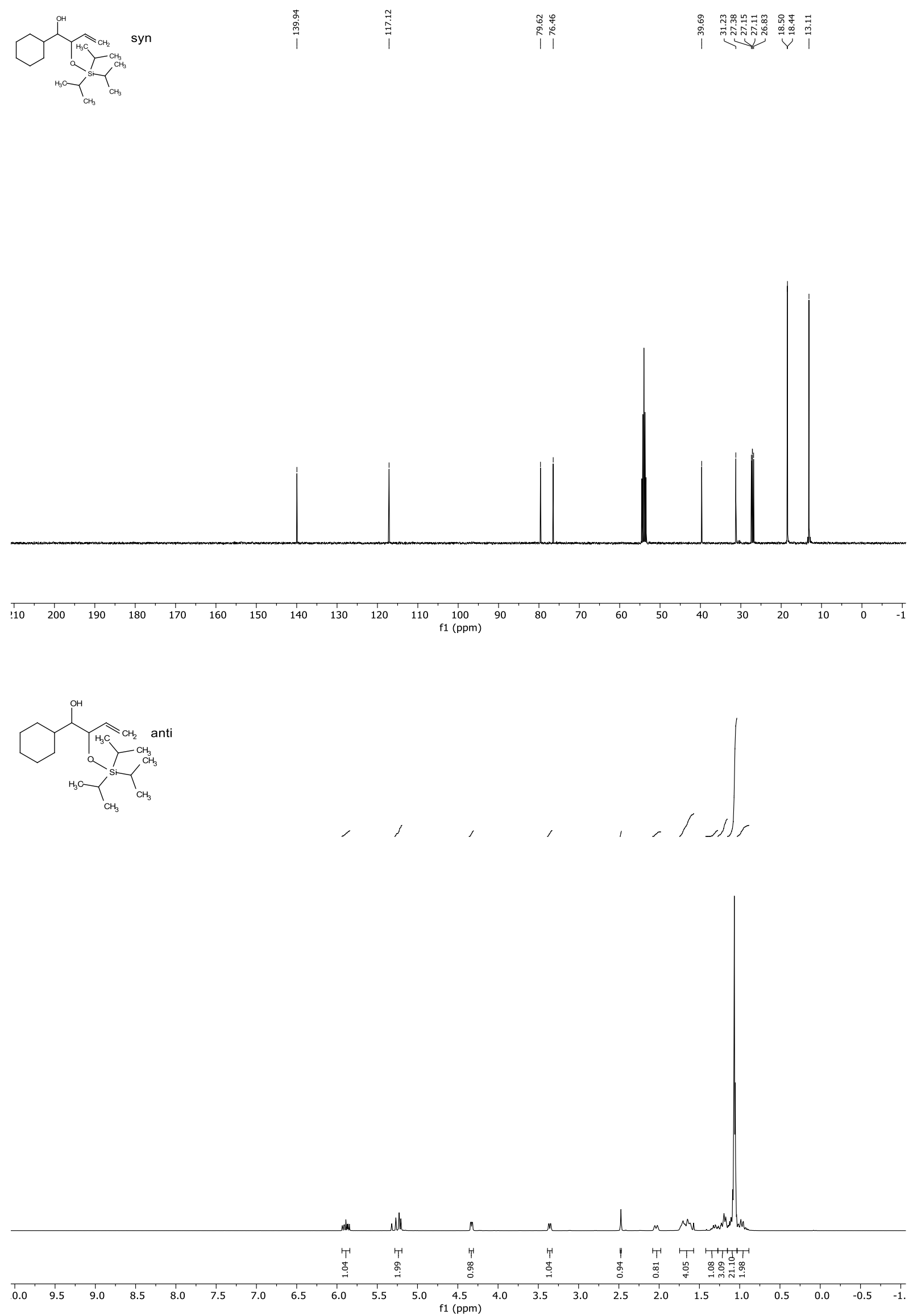

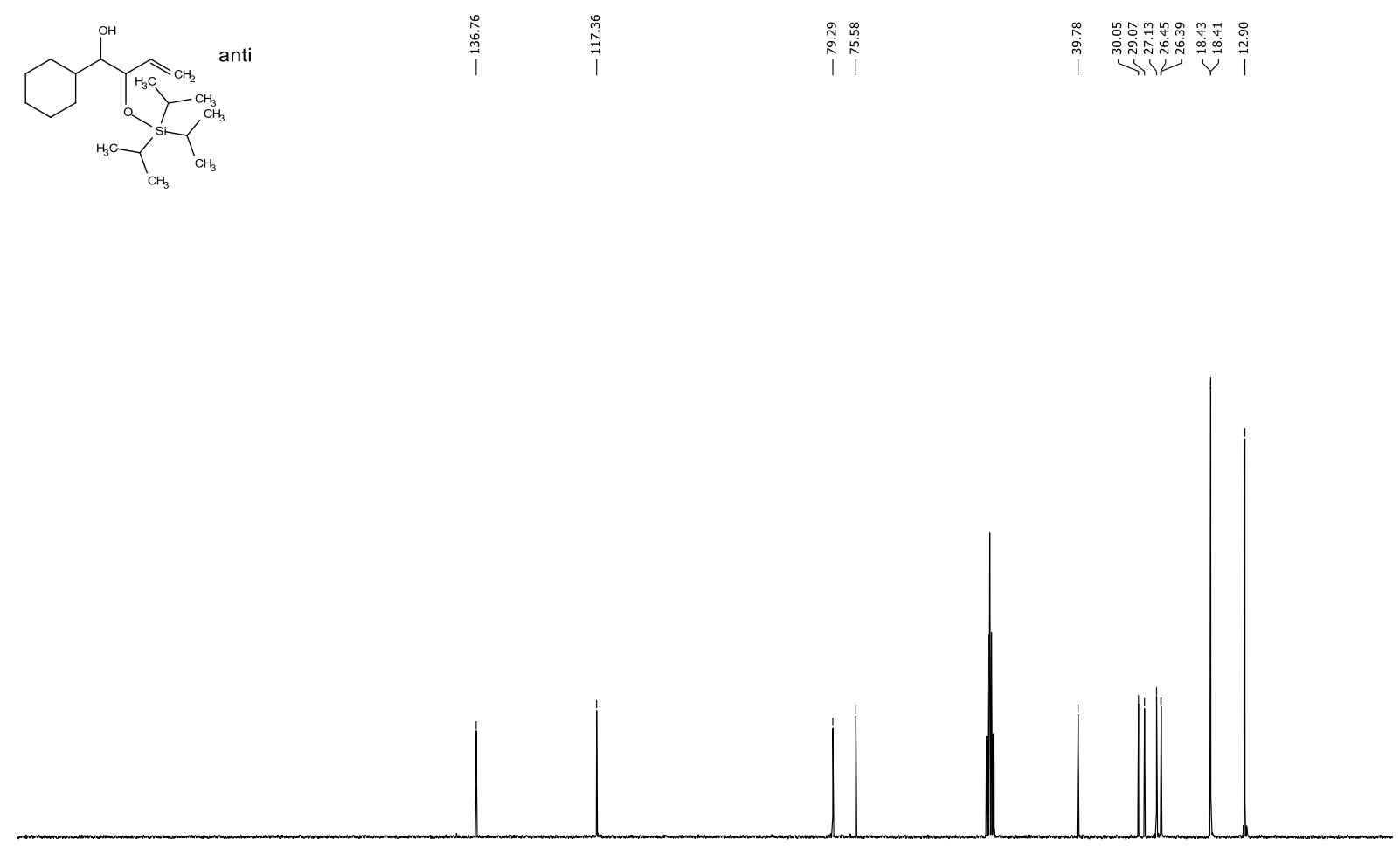

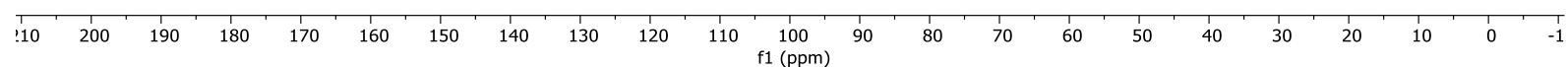<smiles>C=CC(OS(C)(C)C(C)(C)C)C(O)C1CCCCC1</smiles>
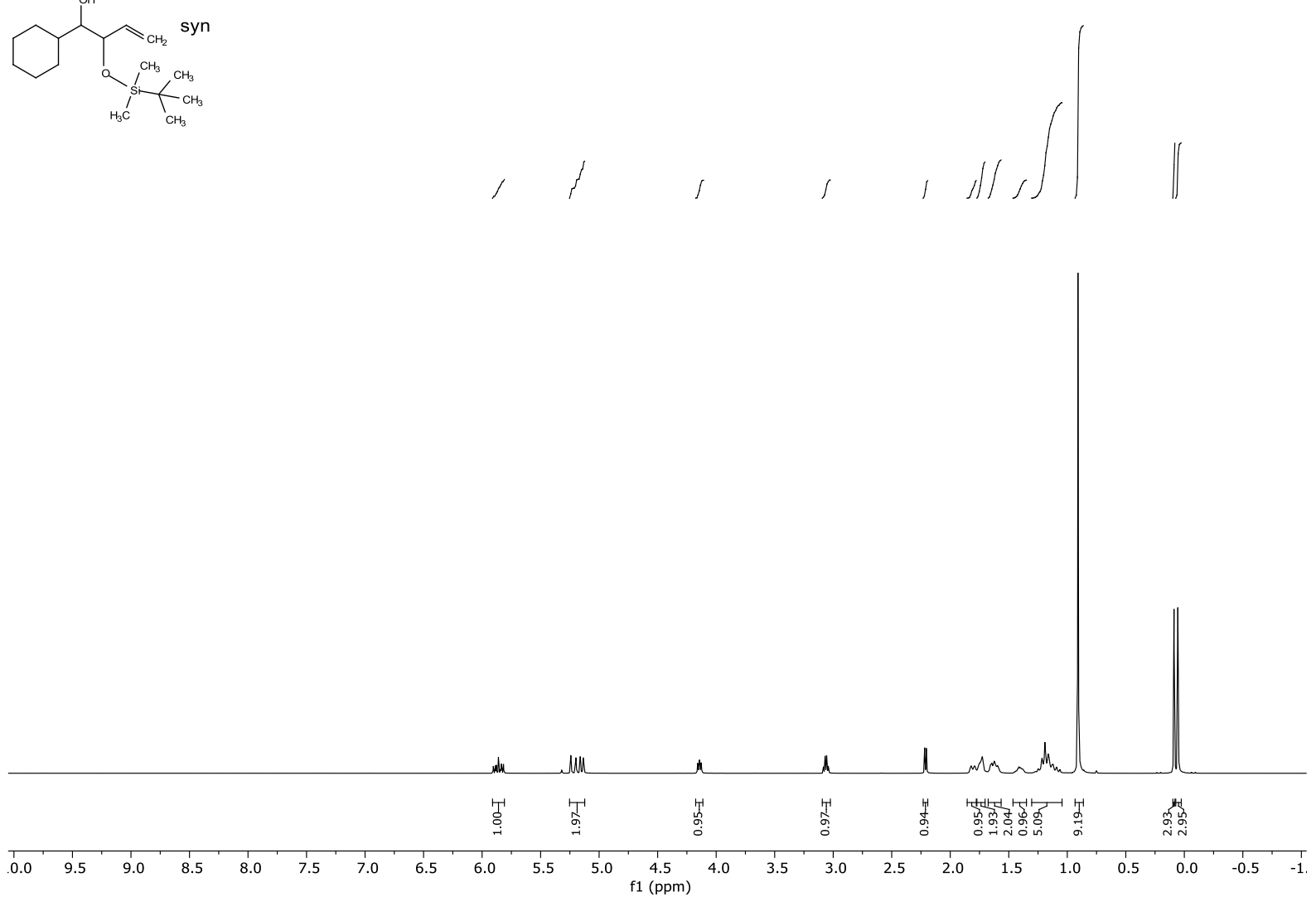

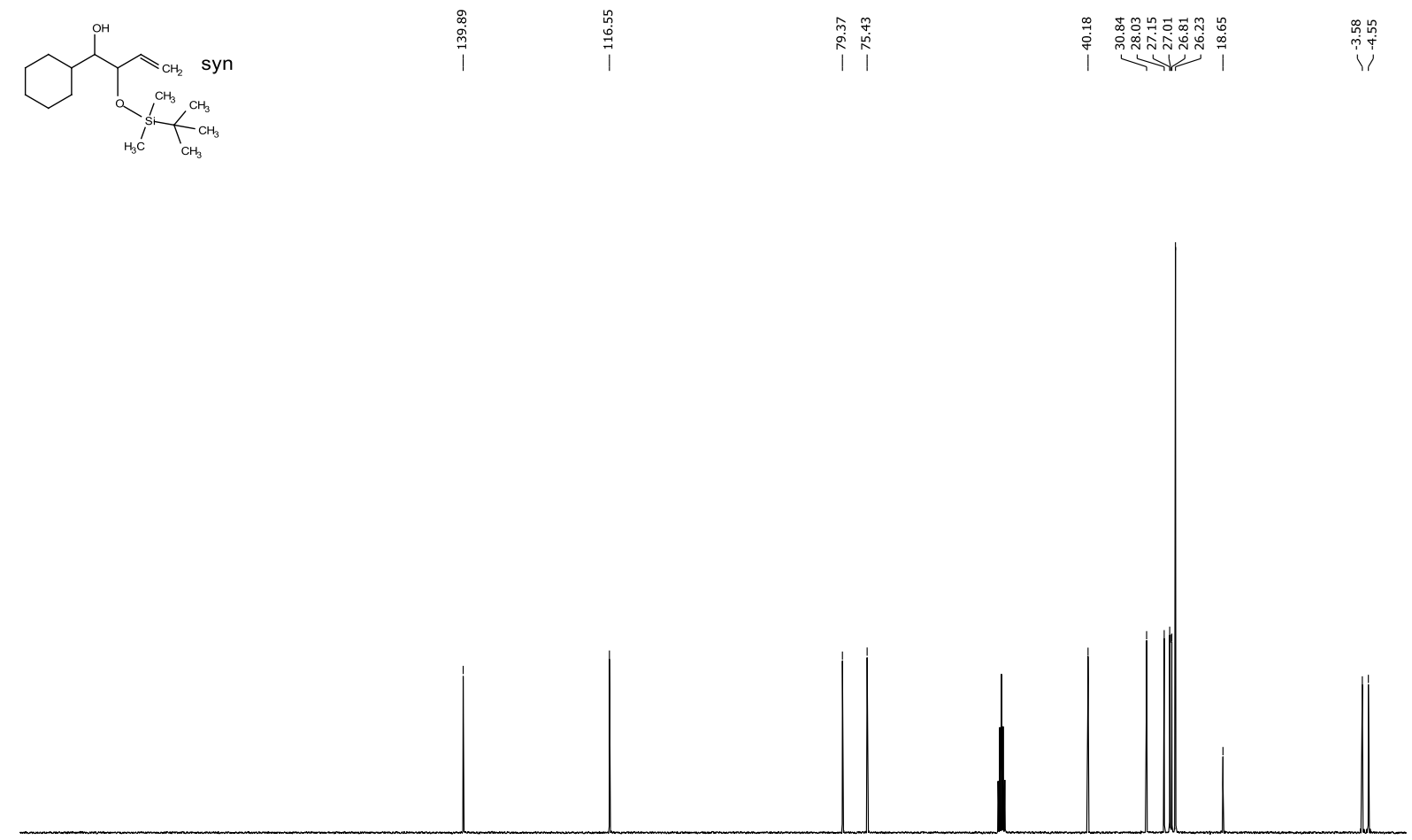

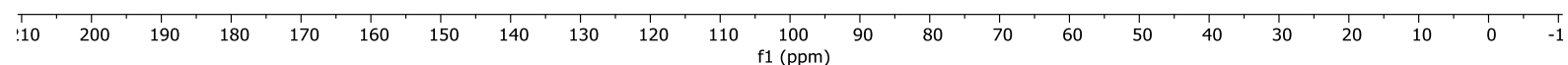
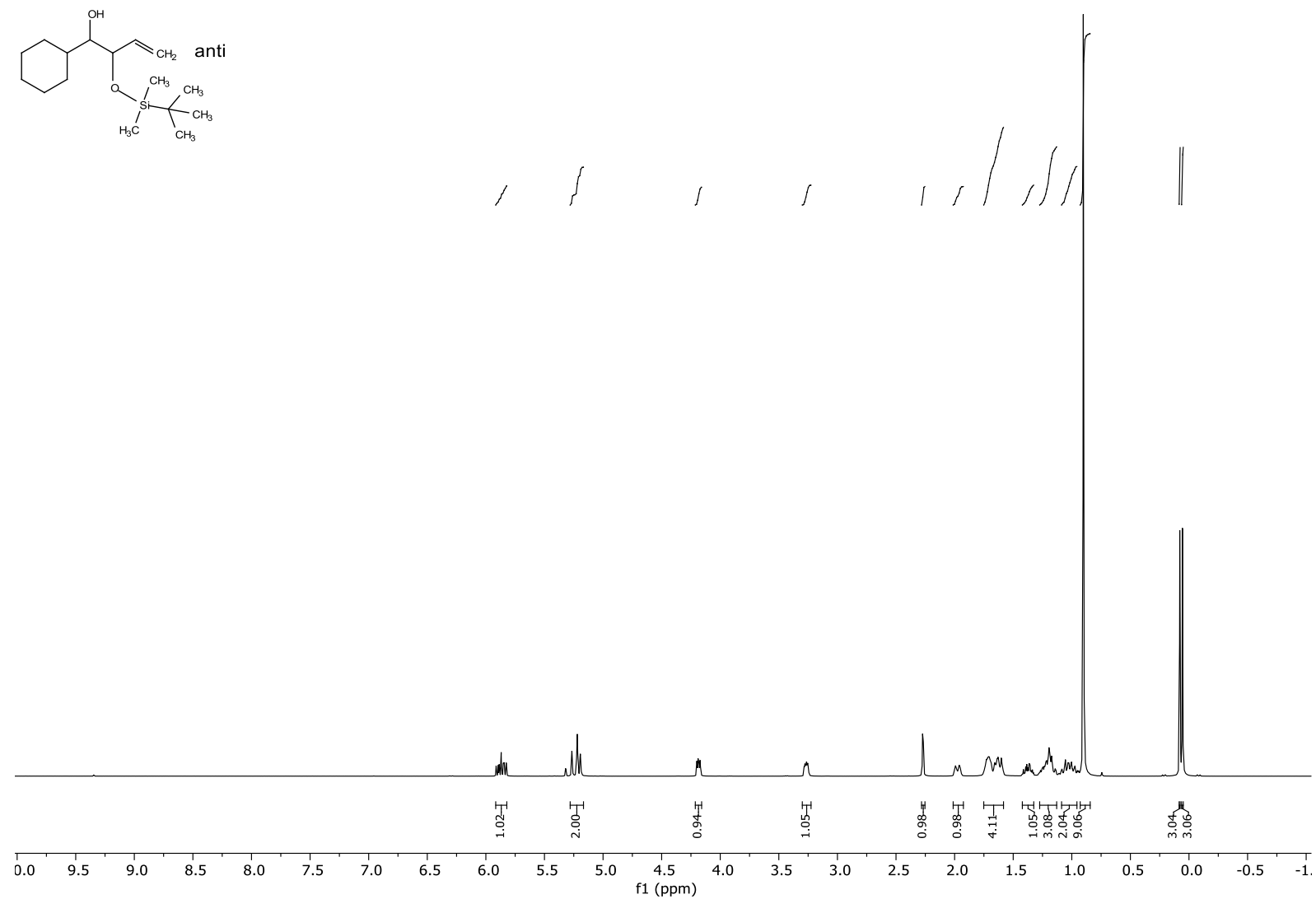

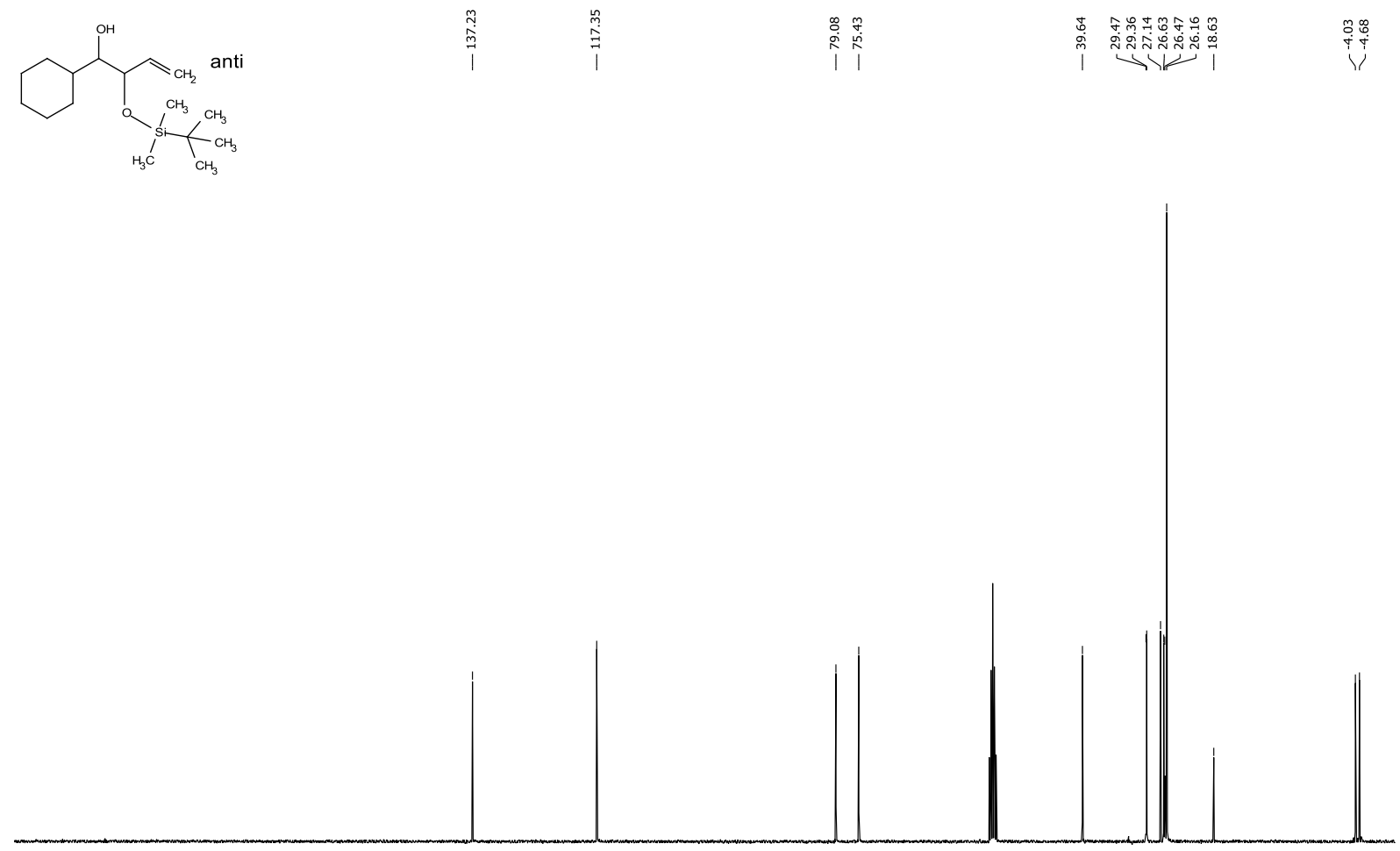

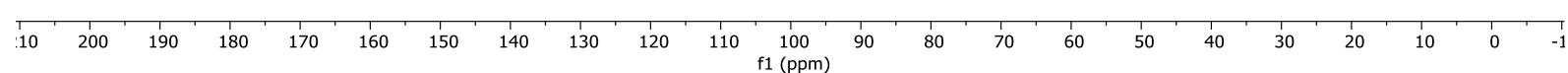
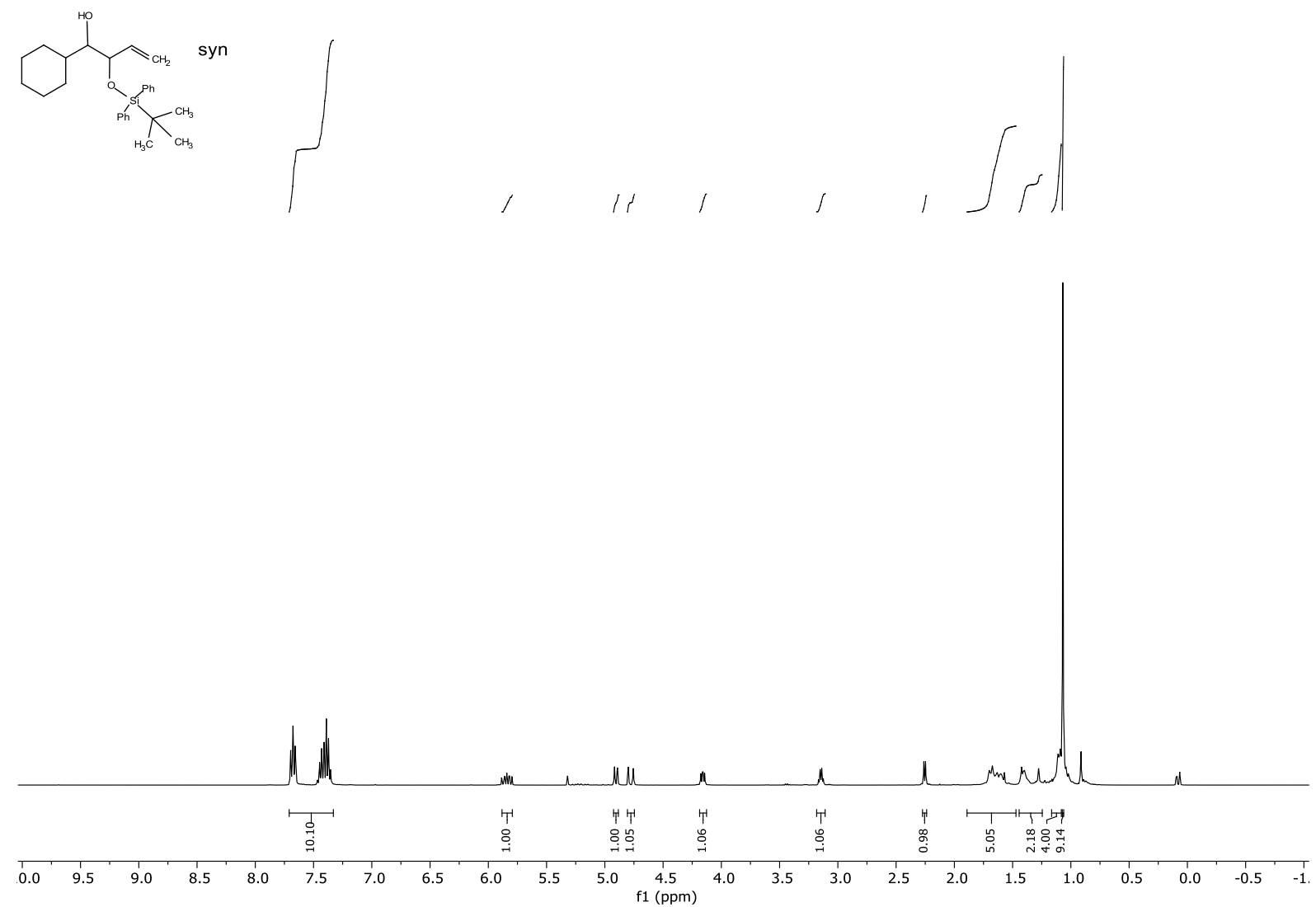


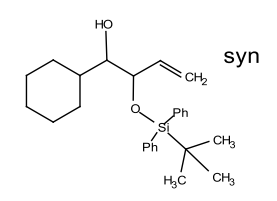

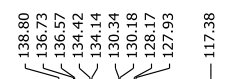
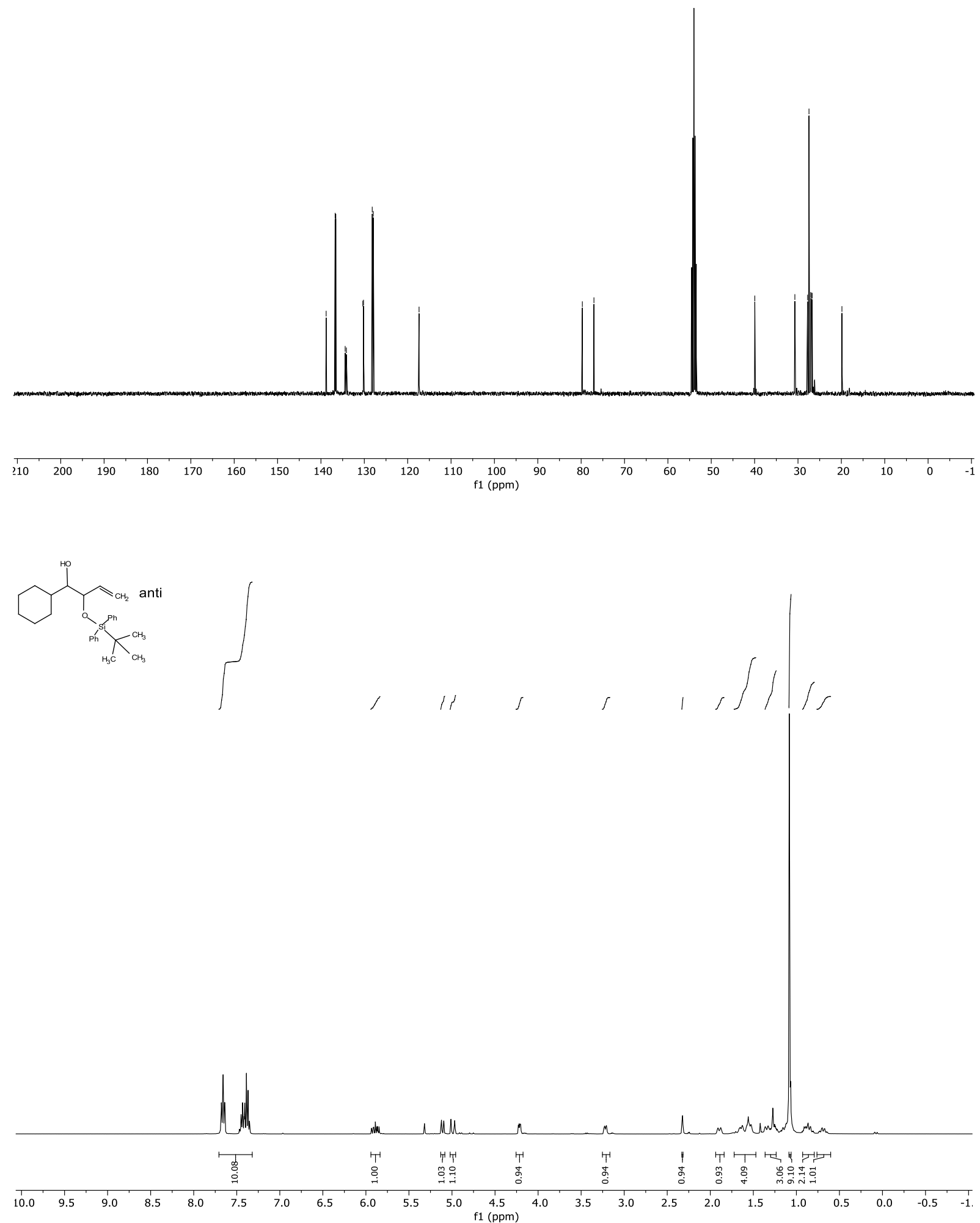


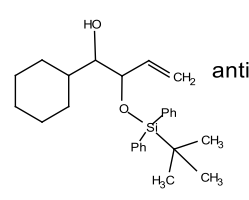

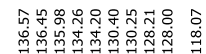

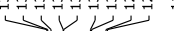

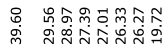

计

$\mathrm{H}_{3} \mathrm{C} \mathrm{CH}_{3}$

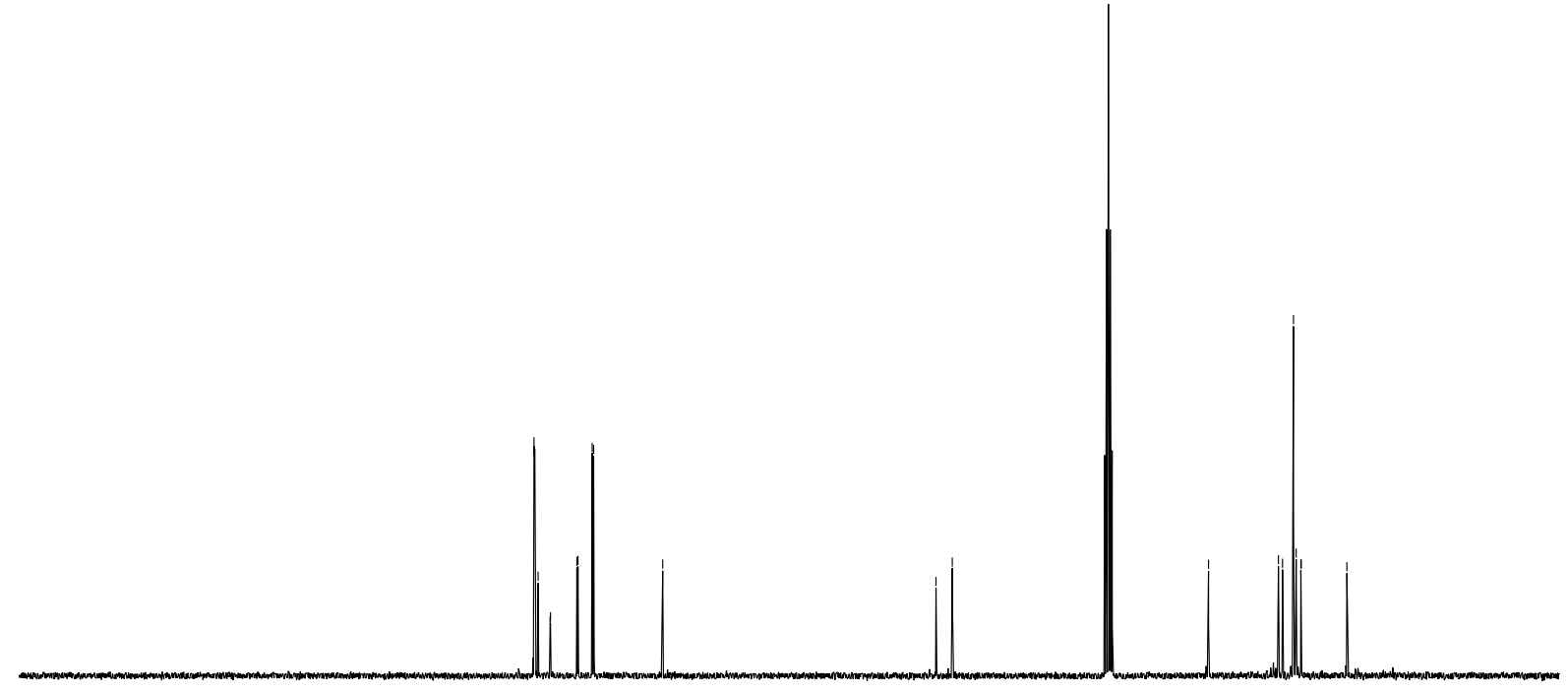

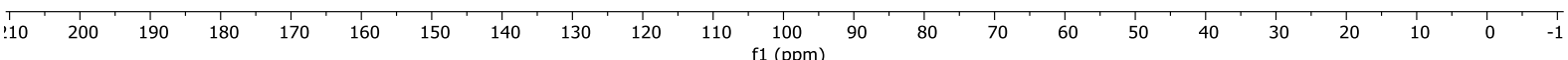
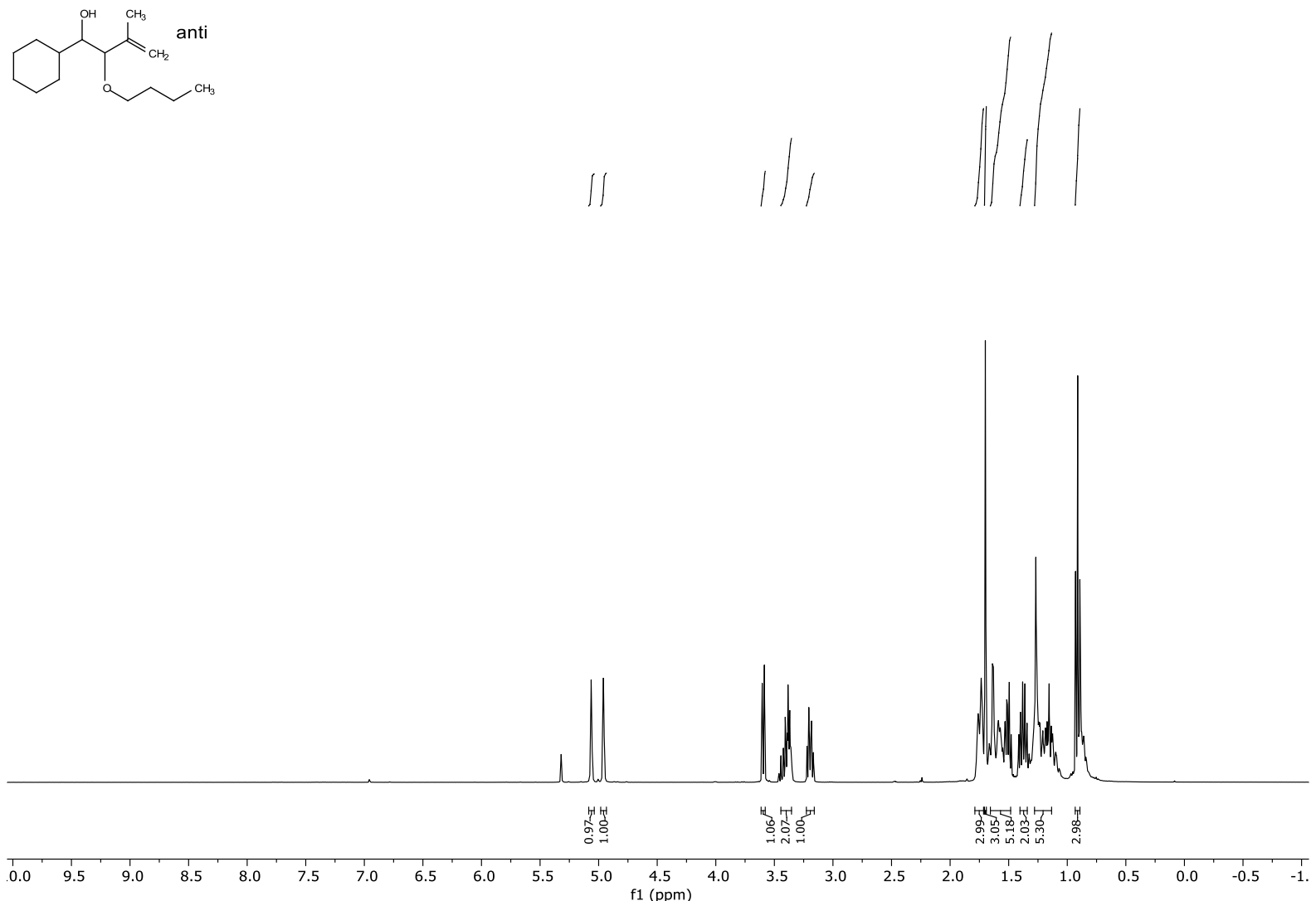

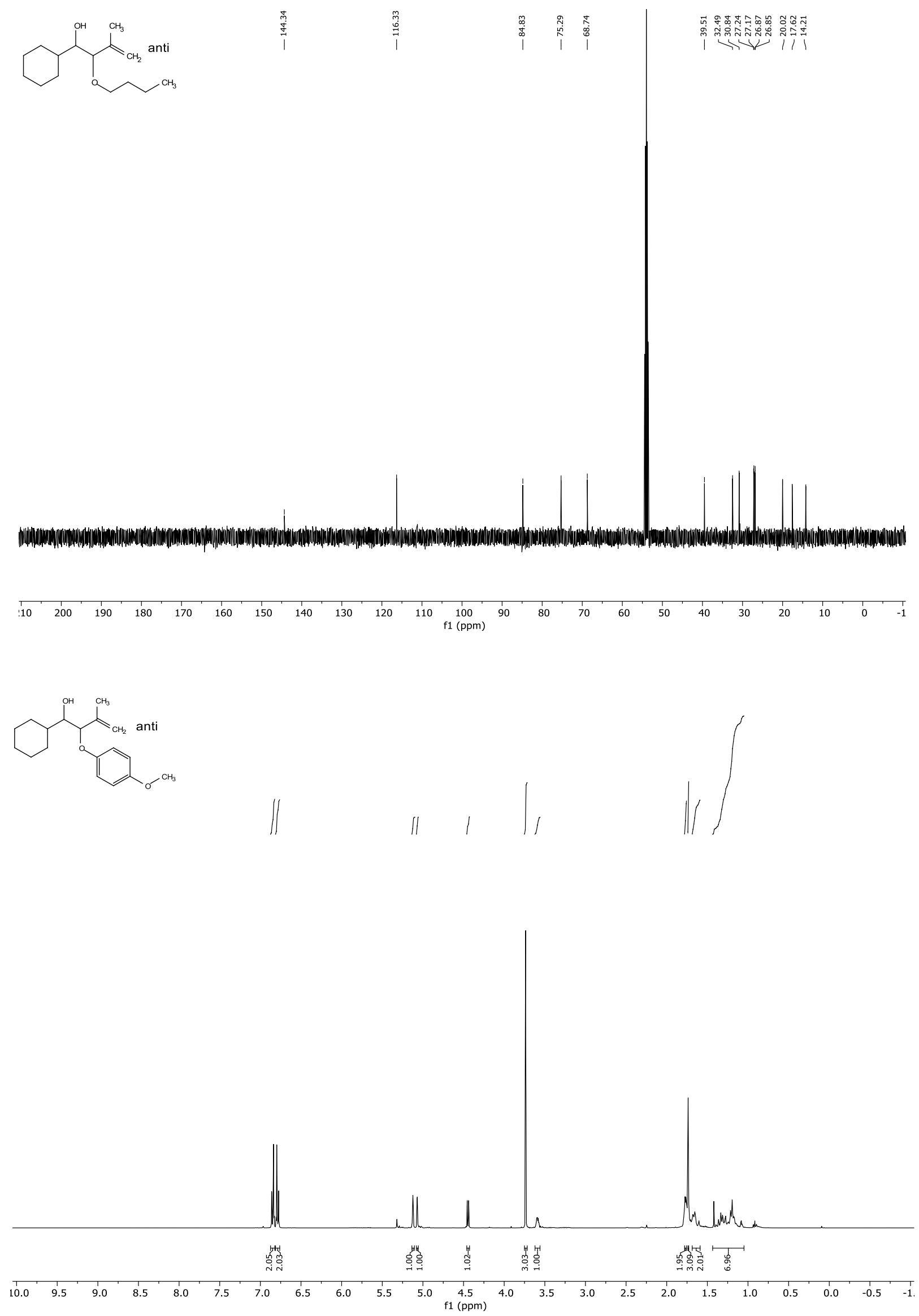

S80 


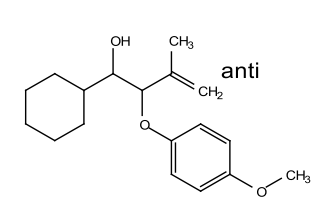

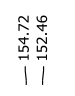

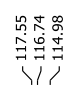

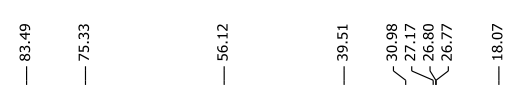
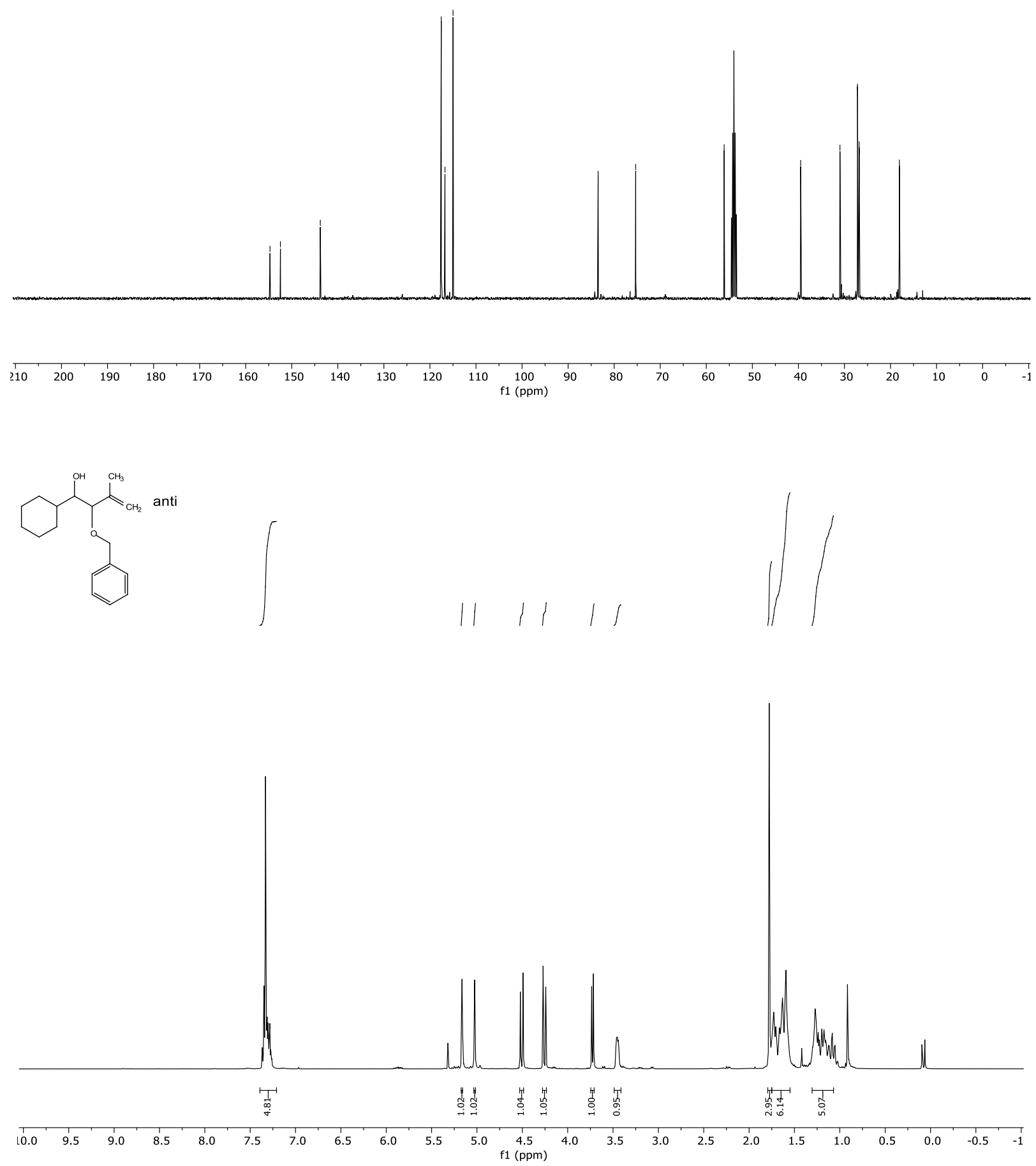

S81 


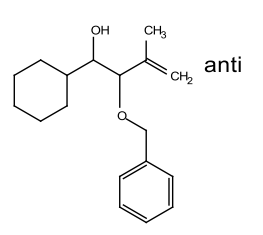

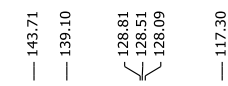

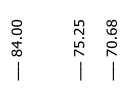

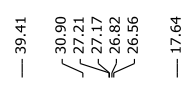

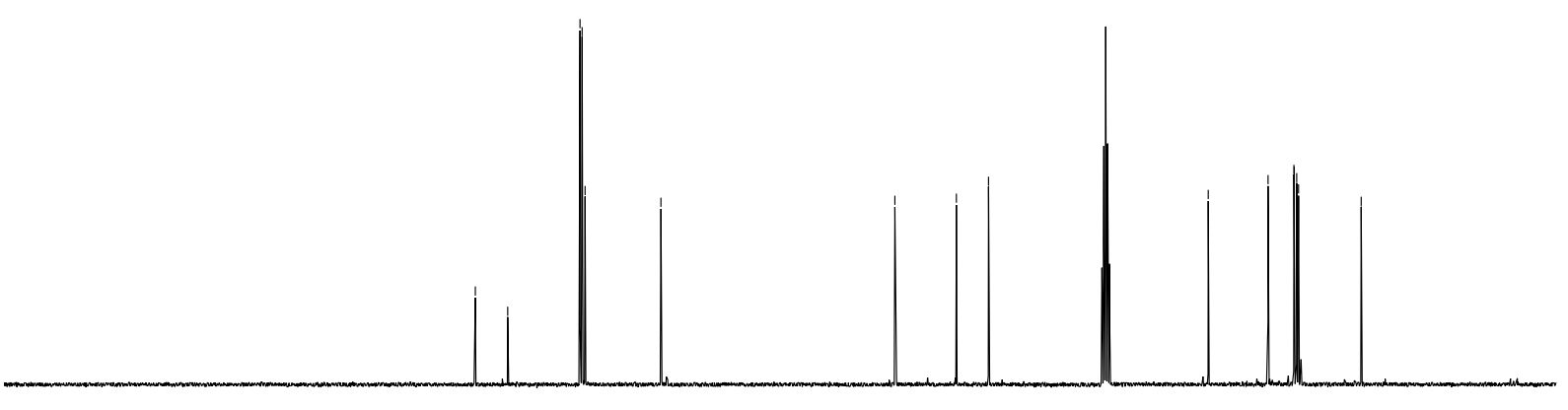

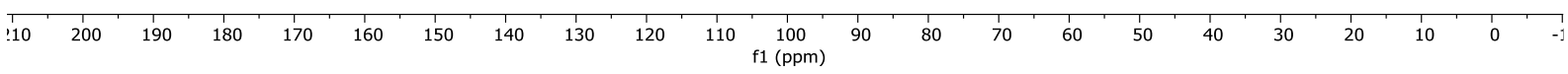
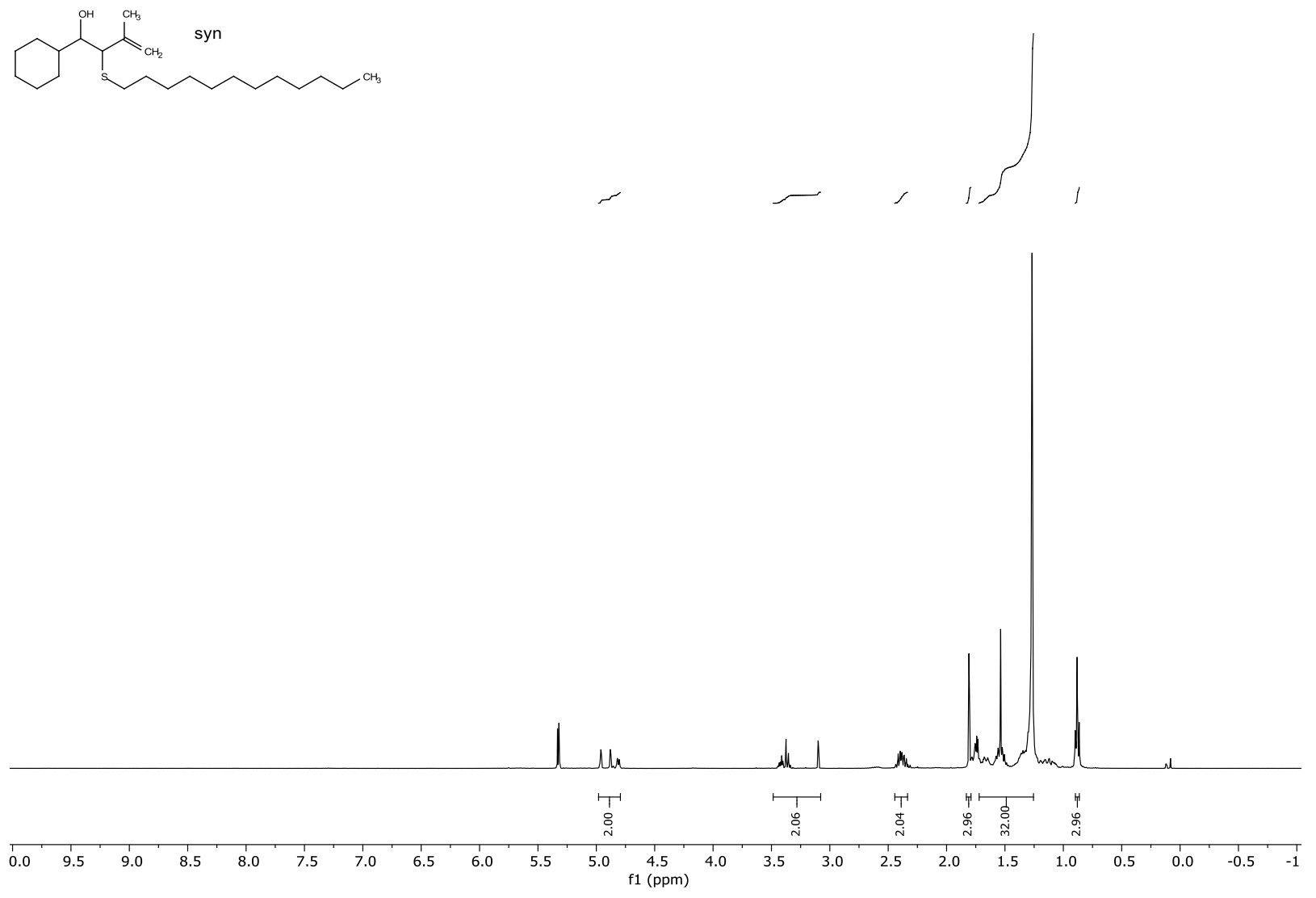

S82 


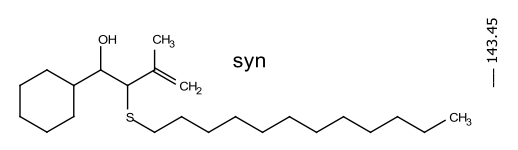

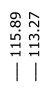

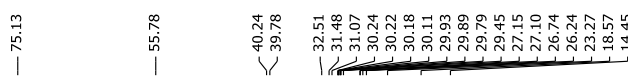

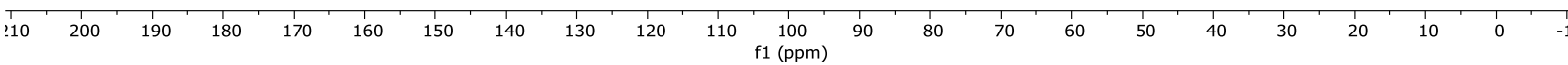
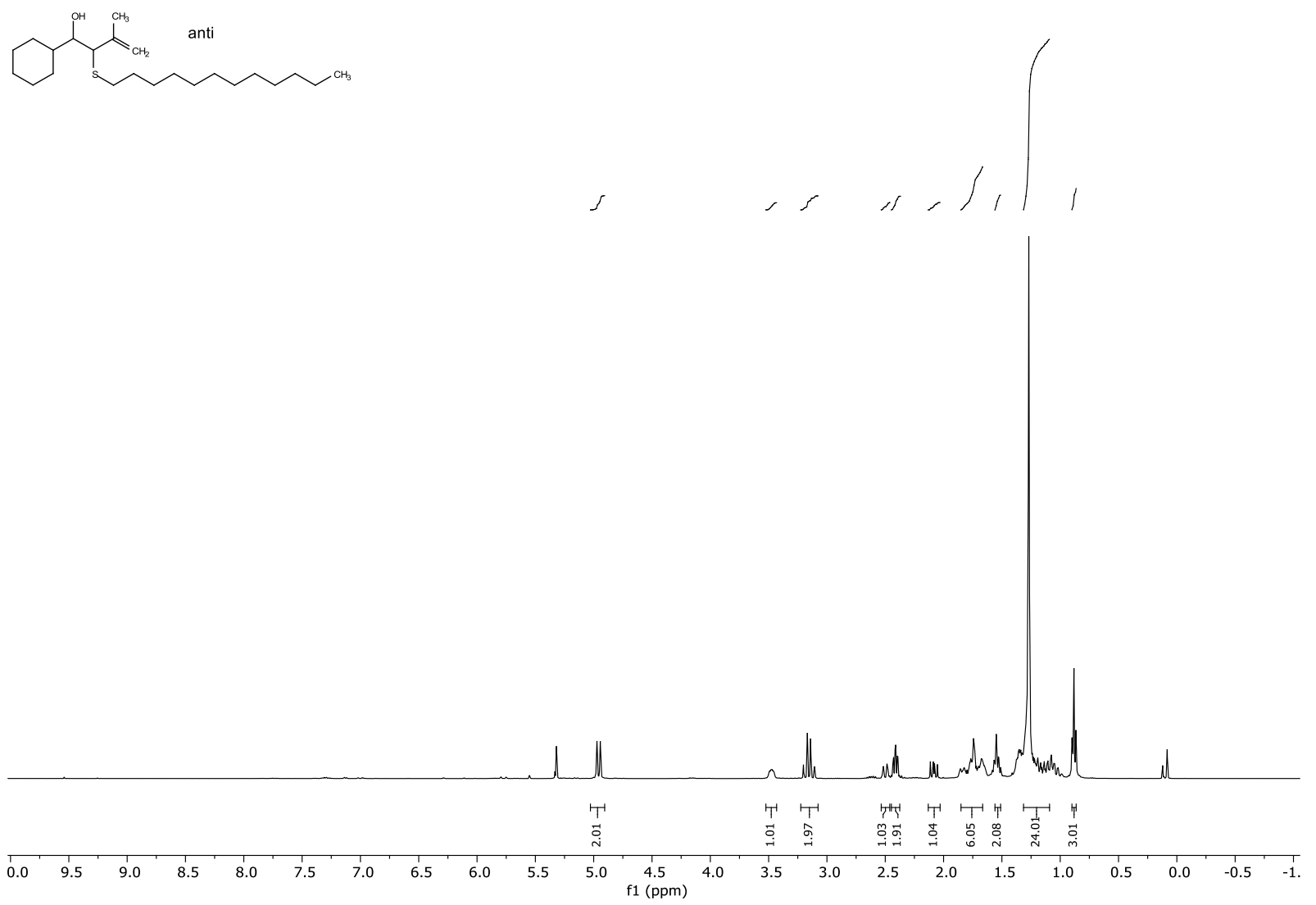

S83 


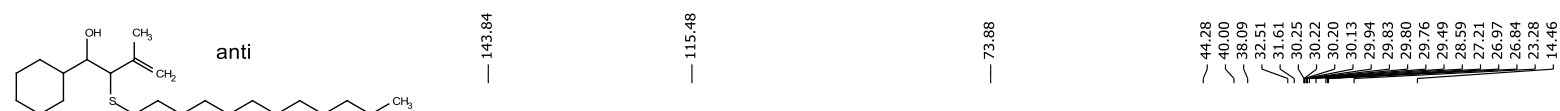

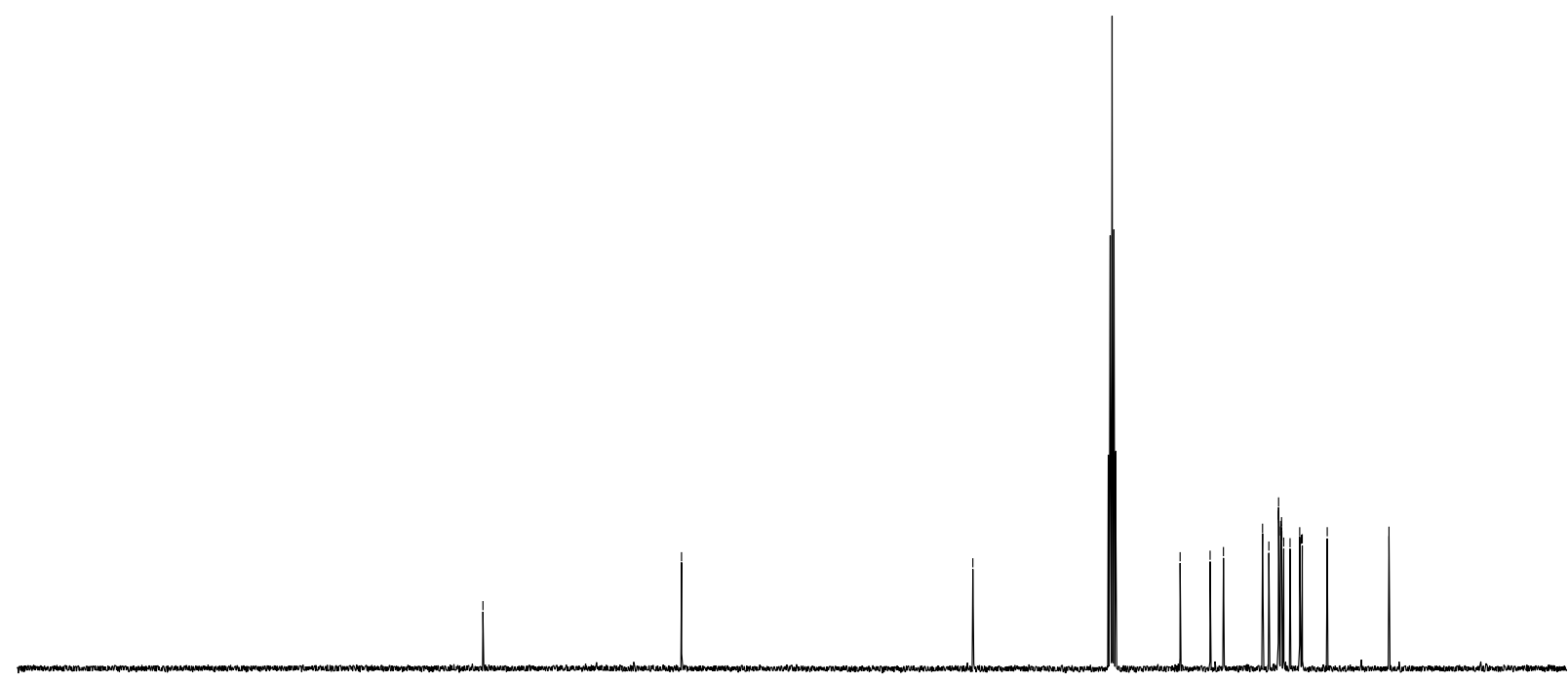

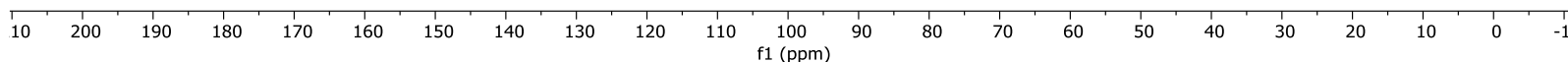<smiles>C=CC(OS(C)(C)C)C(O)C1CCCCC1</smiles>

\section{syn}

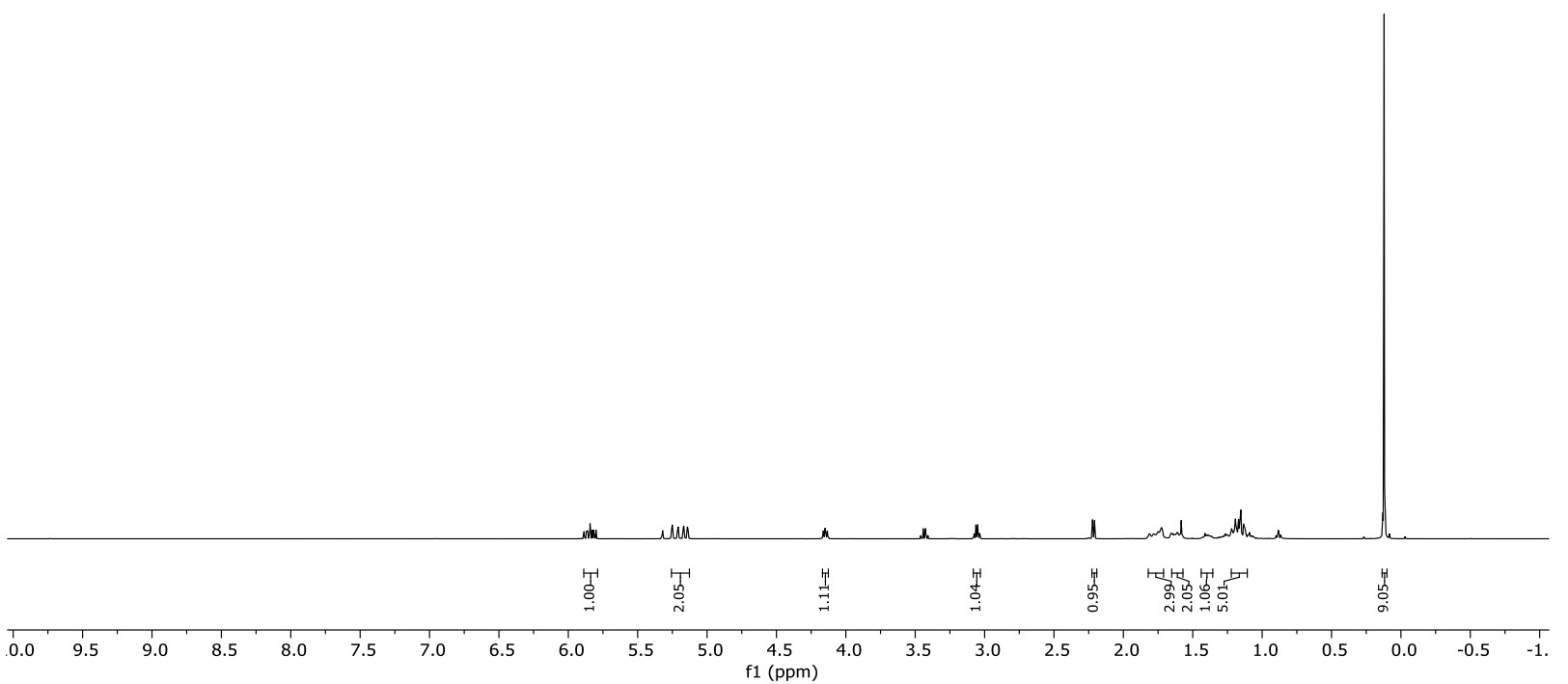




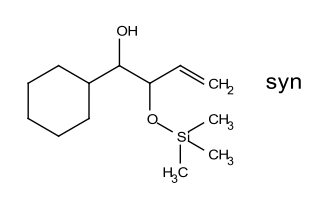

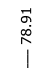

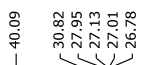

$\stackrel{\circ}{\circ}$

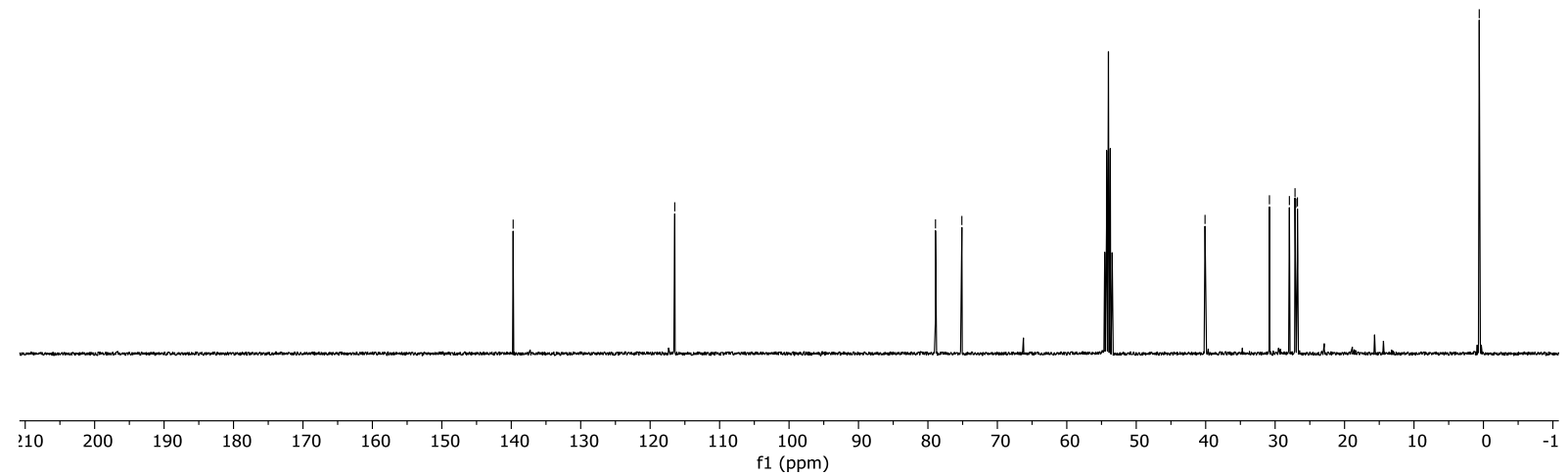

$\overbrace{\substack{\mathrm{S}_{\mathrm{H}}<\mathrm{CH}_{3} \\ \mathrm{CH}_{3} \mathrm{C}}}^{\mathrm{CH}_{3}}$ anti
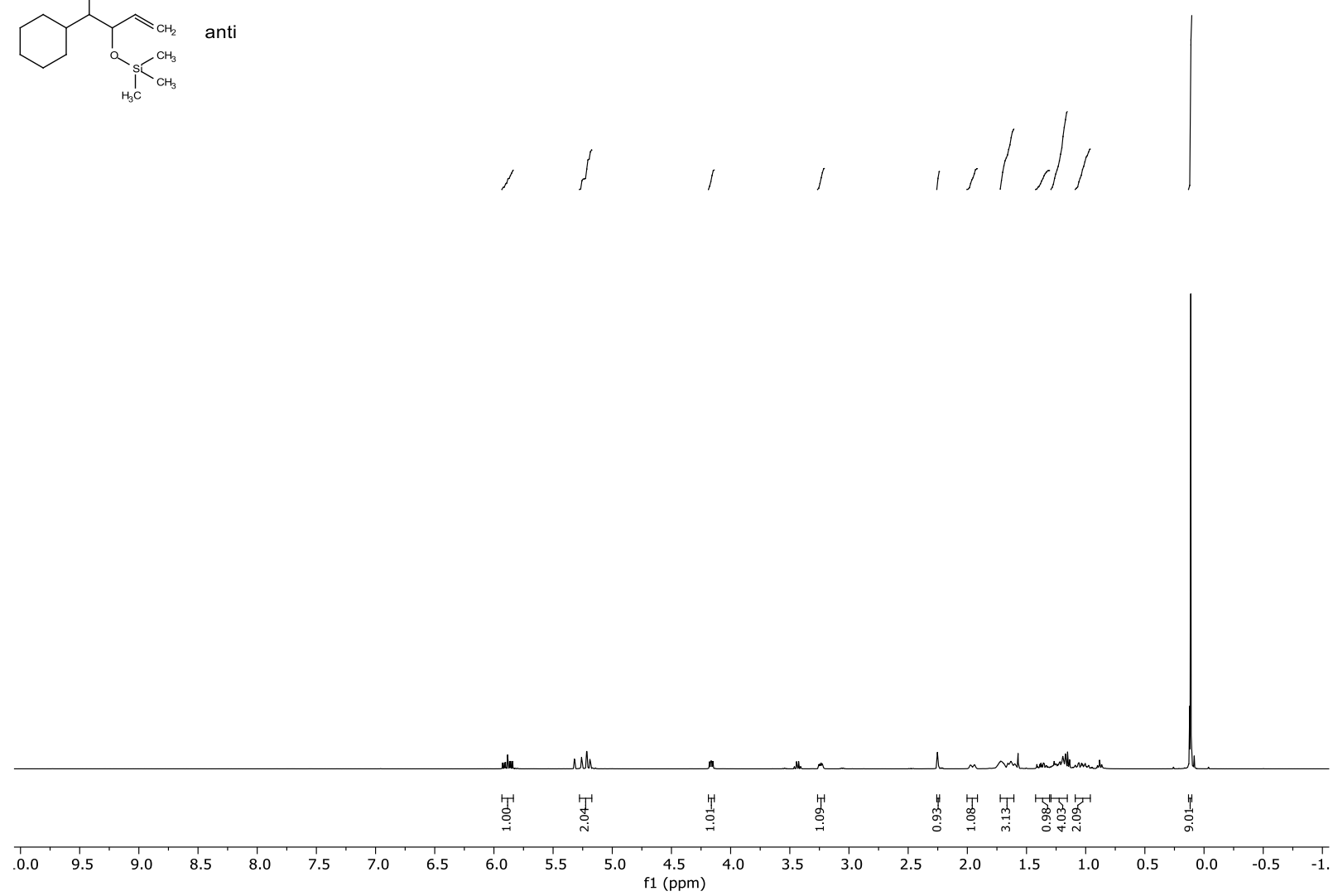


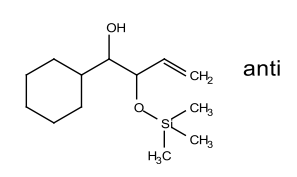

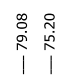
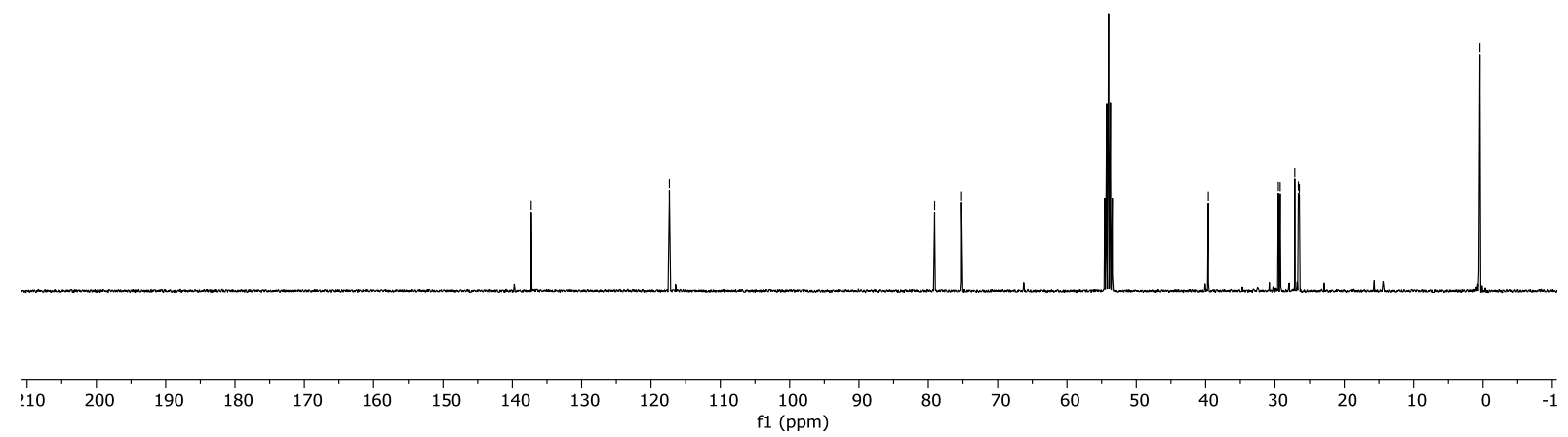

$\overbrace{}^{\text {Ont }}$
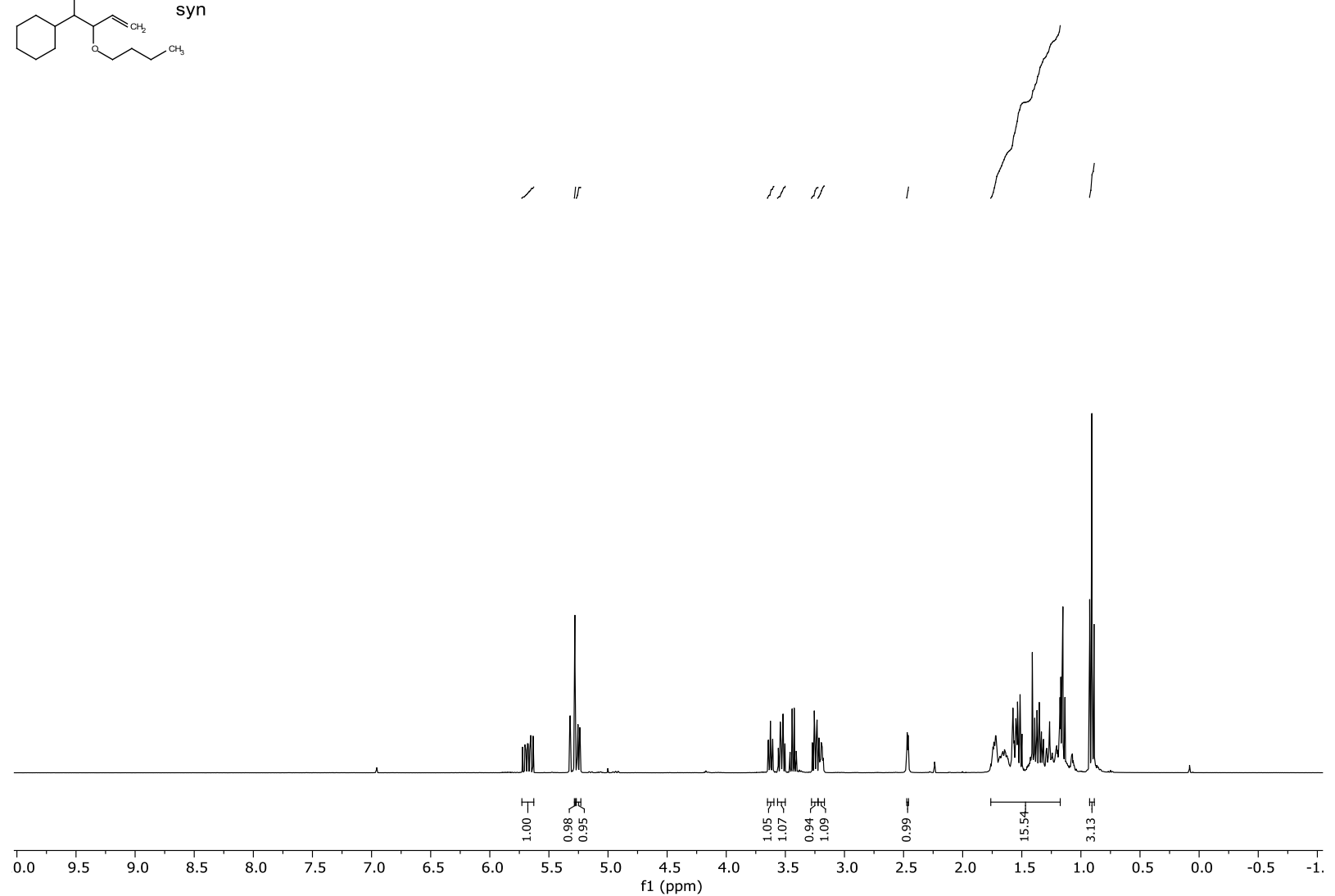


Othan

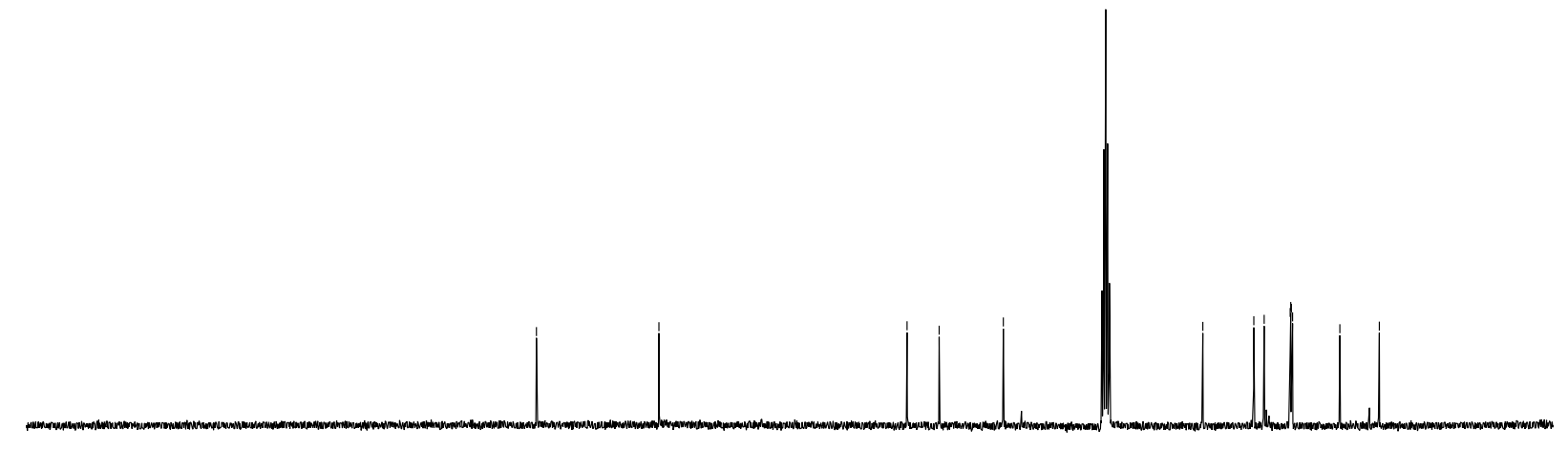

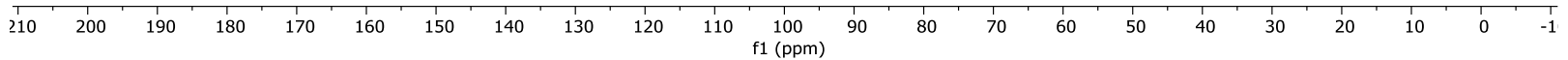

$\overbrace{}^{\text {Canti }}$

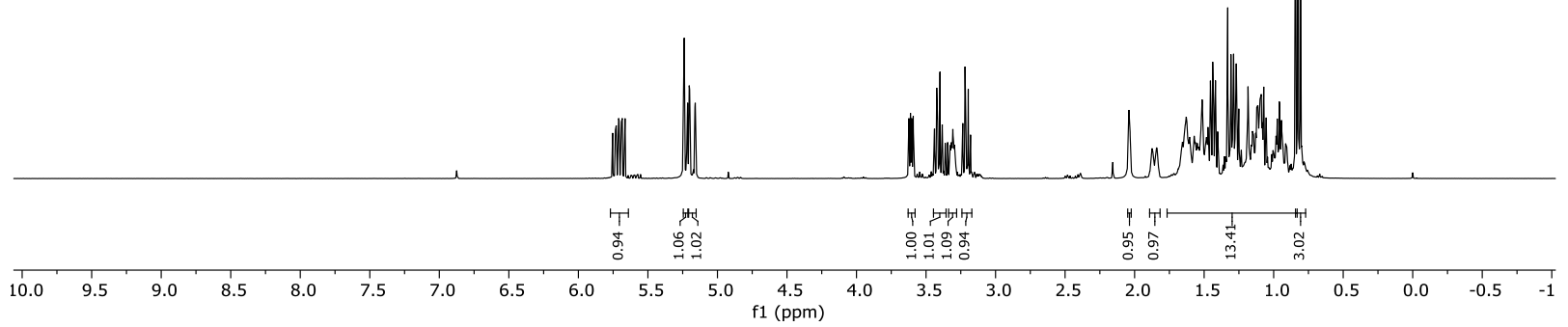




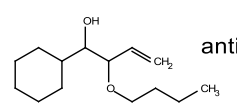

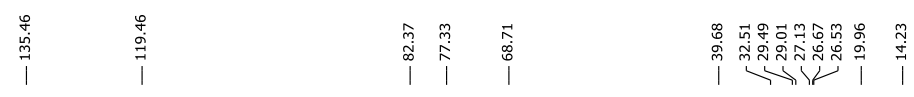

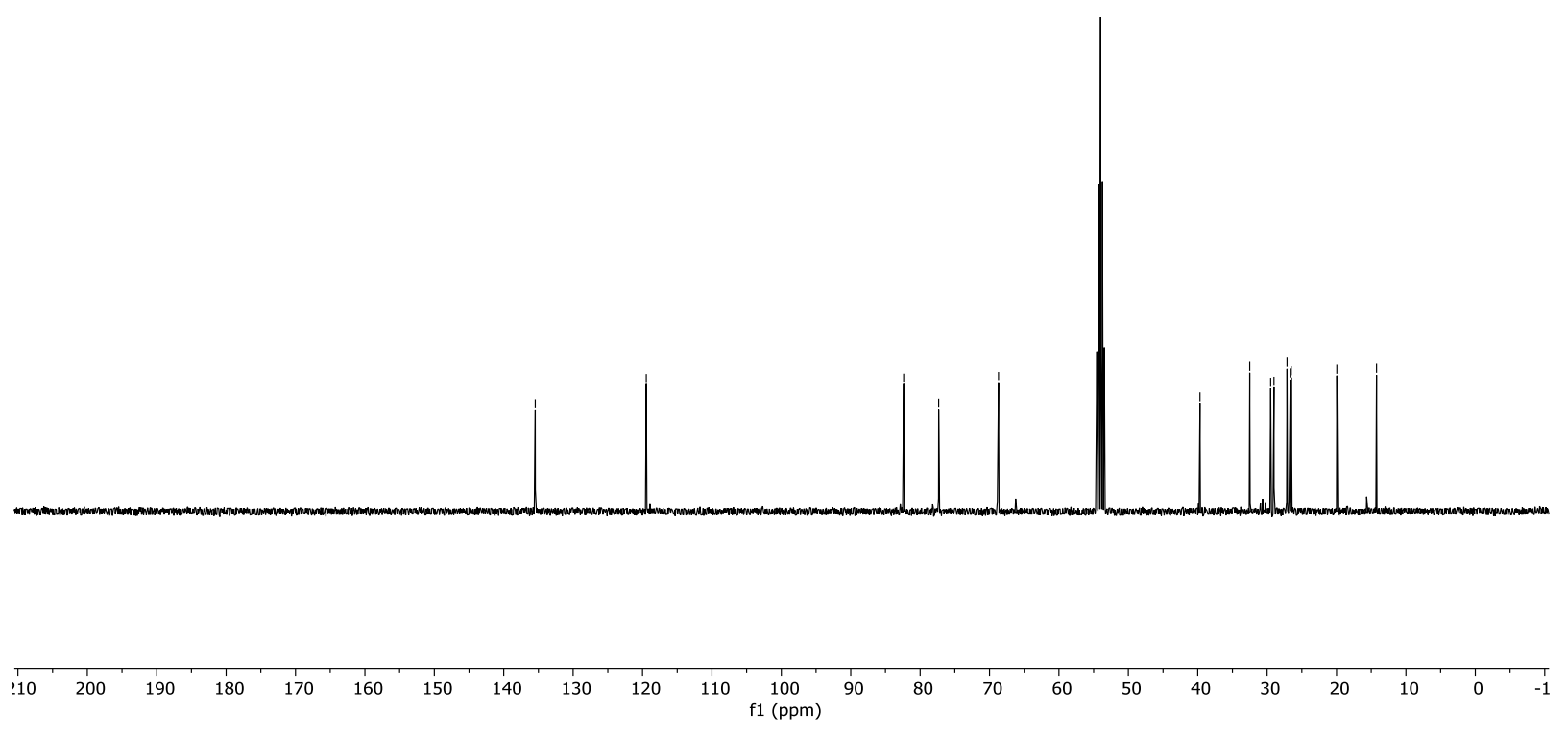

\section{References}

(1) (a) Zhu, Q.; Graff, D. E.; Knowles, R. R. Intermolecular Anti-Markovnikov Hydroamination of Unactivated Alkenes with Sulfonamides Enabled by Proton-Coupled Electron Transfer. J. Am. Chem. Soc. 2018, 140, 741-747; (b) Miller, D. C.; Ganley, J. M.; Musacchio, A. J.; Sherwood, T. C.; Ewing, W. R.; Knowles, R. R. AntiMarkovnikov Hydroamination of Unactivated Alkenes with Primary Alkyl Amines. J. Am. Chem. Soc. 2019, 141, 16590-16594.

(2) Iwasawa, N.; Watanabe, S.; Ario, A.; Sogo, H. Re(I)-Catalyzed Hydropropargylation of Silyl Enol Ethers Utilizing Dynamic Interconversion of VinylideneAlkenylmetal Intermediates via 1,5-Hydride Transfer. J. Am. Chem. Soc. 2018, 140, 7769-7772.

(3) Yang, S.; Zhu, S.; Lu, D.; Gong, Y. Polarity-Reversed Addition of Enol Ethers to Imines under Visible Light: Redox-Neutral Access to Azide-Containing Amino Acids. Org. Lett. 2019, 21, 8464-8468.

(4) Guha, S. K.; Shibayama, A.; Abe, D.; Sakaguchi, M.; Ukaji, Y.; Inomata, K. "Syn-Effect " in the Conversion of (E )- $\alpha, \beta$-Unsaturated Esters into the Corresponding B,y-Unsaturated Esters and Aldehydes into Silyl Enol Ethers. Bull. Chem. Soc. Jpn. 2004, 77, 2147-2157.

(5) Su, C.; Williard, P. G. Isomerization of allyl ethers initiated by lithium diisopropylamide. Org. Lett. 2010, 12, 5378-5381.

(6) Nordmann, G.; Buchwald, S. L. A Domino Copper-Catalyzed C-O Coupling-Claisen Rearrangement Process. J. Am. Chem. Soc. 2003, 125, 4978-4979.

(7) Gao, Y.; Gao, Y.; Wu, W.; Jiang, H.; Yang, X.; Liu, W.; Li, C.-J. Palladium-Catalyzed Tandem Oxidative Arylation/Olefination of Aromatic Tethered Alkenes/Alkynes. Chem. Eur. J. 2017, 23, 793-797.

(8) Krompiec, S.; Kuźnik, N.; Urbala, M.; Rzepa, J. Isomerization of Alkyl Allyl and Allyl Silyl Ethers Catalyzed by Ruthenium Complexes. J. Mol. Catal. A. Chem. 2006, 248, 198-209.

(9) Lin, S.; Lies, S. D.; Gravatt, C. S.; Yoon, T. P. Radical Cation Cycloadditions Using Cleavable Redox Auxiliaries. Org. Lett. $2017,19,368-371$.

(10) Mukherjee, S.; Patra, T.; Glorius, F. Cooperative Catalysis: A Strategy To Synthesize Trifluoromethyl-thioesters from Aldehydes. ACS Catal. 2018, 8, 58425846.

(11) Cismesia, M. A.; Yoon, T. P. Characterizing Chain Processes in Visible Light Photoredox Catalysis. Chem. Sci. 2015, 6, 5426-5434.

(12) A new sensitive chemical actinometer - II. Potassium Ferrioxalate as a Standard Chemical Actinometer. Proc. R. Soc. Lond. 1956, $235,518-536$.

(13) (a) Kuhn, H. J.; Braslavsky, S. E.; Schmidt, R. Chemical actinometry (IUPAC Technical Report). Pure Appl. Chem. 2004, 76, 2105-2146; (b) Montalti, M.; Credi, A.; Prodi, L.; Gandolfi, M. T.; Michl, J.; Balzani, V. Handbook of photochemistry, Third edition; CRC/Taylor \& Francis: Boca Raton, 2006. 
(14) Wegner, E. E.; Adamson, A. W. Photochemistry of Complex lons. III. Absolute Quantum Yields for the Photolysis of Some Aqueous Chromium(III) Complexes. Chemical Actinometry in the Long Wavelength Visible Region. J. Am. Chem. Soc. 1966, 88, 394-404.

(15) Pitzer, L.; Schäfers, F.; Glorius, F. Rapid Assessment of the Reaction-Condition-Based Sensitivity of Chemical Transformations. Angew. Chem. Int. Ed. 2019, $58,8572-8576$.

(16) Gensch, T.; Teders, M.; Glorius, F. Approach to Comparing the Functional Group Tolerance of Reactions. J. Org. Chem. 2017, 82, 9154-9159.

\section{Author Contributions}

Felix Schäfers: Data curation, formal analysis, investigation, project administration, writing of original draft.

Linda Quach: Data curation, formal analysis, investigation, validation.

J. Luca Schwarz: Data curation, formal analysis, validation.

Mar Saladrigas: Data curation, formal analysis, investigation, validation.

Constantin G. Daniliuc: X Ray Measurement.

Frank Glorius: Funding acquisition, project administration, validation, writing of original draft. 